\title{
Nonlinear Formulations of a Four-Node Quasi-Conforming Shell Element
}

\author{
Gilson Rescober Lomboy • Songsak Suthasupradit • \\ Ki-Du Kim • E. Oñate
}

Received: 8 January 2009 / Accepted: 8 January 2009 / Published online: 21 February 2009

(C) CIMNE, Barcelona, Spain 2009

\begin{abstract}
The quasi-conforming technique was introduced in the 1980's to meet the challenge of inter-elements conforming problems and give a unified treatment of both conforming and nonconforming elements. While the linear formulation is well established, the nonlinear formulation based on the quasi-conforming technique that includes geometric and material nonlinearity is presented in this paper. The formulation is derived in the framework of an updated Lagrangian stress resultant, co-rotational approach. The geometric nonlinear formulation provides solutions to buckling and postbuckling behaviour while the material nonlinear formulation considers the spread of plasticity within the element while maintaining an explicit construction of element matrices. Aside from the elasto-plastic constitutive relation, formulations on laminate composites and reinforced concrete are also presented.

The formulations of laminate composite and reinforced concrete material are present based on the layer concept, the material properties can vary throughout the thickness and across the surface of a shell element. The various failure criteria for laminate composite are included in the formulation which makes it possible to analyses the progressive failure
\end{abstract}

\section{G.R. Lomboy}

Department of Civil, Construction, and Environmental

Engineering, Iowa State University, Ames, IA 50011, USA

S. Suthasupradit · K.-D. Kim $(\bowtie)$

Department of Civil and Environmental System Engineering,

Konkuk University, Seoul, 130-702, Korea

e-mail:kimkd@konkuk.ac.kr

E. Oñate

International Center for Numerical Methods in Engineering (CIMNE), Universidad Politecnica de Cataluna, Edificio C1 Campus Nord, 08032 Barcelona, Spain of fibre and matrix. For the reinforced concrete material, the nonlinearities as a result of tensile cracking, tension stiffening between cracks, the nonlinear response of concrete in compression, and the yielding of the reinforcement are considered. The steel reinforcement is modeled as a bilinear material with strain hardening.

\section{Introduction}

\subsection{Development of the Quasi-Conforming Technique}

Tang et al. [149] introduced the quasi-conforming technique. The technique was developed to meet the challenge of inter-elements conforming problems, give a unified treatment of both conforming and nonconforming elements, and to generate new elements. The element formulation involved the string net function that is a generalized spline function on an arbitrary two-dimensional domain. They emerge in the integration of the piecewise polynomials on the element boundaries. Similar to Pian's [108] work on the assumed stress formulation, polynomials are used to describe the strain field. The displacement functions appear in the derivation of the strain-displacement matrix with boundary integrals. This means that the displacement function is only explicitly expressed along the boundaries of the element. In 1983, Tang et al. presented the quasi-conforming technique again, this time; the emphasis was made on the improvement of the displacement interpolation function. Different types of 2D elements, plane, plates and shells were used to demonstrate how the quasi-conforming technique is used. It is also mentioned that $3 \mathrm{D}$ cases are straightforward. However, the examples are all limited to linear formulations. Tang et al. [150] then showed that the quasi-conforming element technique is a multivariate finite element method that 
it includes the compatible, incompatible and hybrid stress model as its special cases. They have also showed the connection between this technique and Hu-Washizu principle.

The quasi-conforming technique has also been used to employ penalty functions. In cases in which additional constraints have to be imposed on a variational function, penalty function methods can be used to remove the constraint equations. Tang and Liu [152] showed the implementation of penalty finite element methods by utilizing the quasiconforming element technique.

The development of nonlinear quasi-conforming elements took some time. In 1993, Guan and Tang [54] presented a geometrically nonlinear quasi-conforming FEM based on the quasi-conforming finite element. The incremental principle of stationary potential energy was first discussed, then, the formulation process of the nonlinear quasiconforming FEM is given. In this case, only one form of weighing functions is used in the enforcement of the equality of the actual full strain tensor with the approximate, as opposed to the formulation presented in this thesis, which proposes different weighing functions for the linear and nonlinear parts of the full Green strain tensor. Qin [112] also presented a nonlinear formulation of the quasi-conforming technique. It was presented using the Hu-Washizu varia-

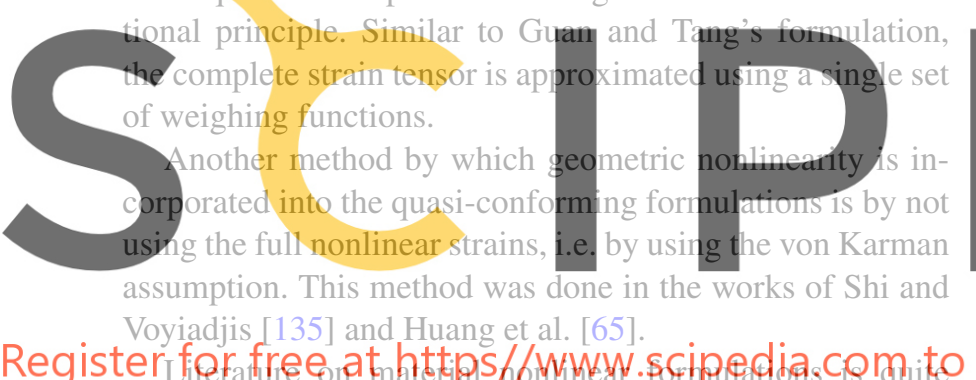

rare. It could be because the development of plasticity in an element is generally observed at Gauss points, and quasiconforming elements are usually derived explicitly, without using numerical integration. Shi and Voyiadjis [135] have been able to successfully formulate an elasto-plastic quasiconforming formulation without the use of Gauss points, but instead used plastic nodes.

Of late, Voyiadjis and Woelke [162] and Woelke et al. [173] presented studies on elasto-plasticity of shells, where the shells were formulated using the quasi-conforming technique. To incorporate the formulated elasto-plastic theories into the shell element formulation, a plastic node concept was used. The plasticity is concentrated at the nodes, the nodes become plastic while the interior of the element remains elastic. The geometric nonlinearity is based on the von Karman assumption. The first article discusses thick shells, while the second has emphasis on damage due to micro-voids for thin and thick shells. Also, based on this study, Kim and Lomboy [82] presented a co-rotational quasi-conforming an elasto-plastic formulation of a fournode stress resultant shell element for non-linear analysis of plate and shell structures. In this case, the spread of plasticity within the element is considered by providing an explicit function of the elasto-plastic stress field.

There is much more literature available on the quasiconforming technique, however, they have been written in Chinese or simply inaccessible to the author. For the purpose of reference for the reader and researchers who may wish to continue studies on the quasi-conforming technique, some of these literatures have been included in the references. These literatures are important to be mentioned and included in this report, because none of them seem to indicate formulations of the quasi-conforming technique that treats geometric and material nonlinearity.

\subsection{Quasi-Conforming Elements}

\section{Since the quasi-conforming technique originated at the Dalian Institute of Technology, the first and majority of lit- erature are in Chinese, published in the local journal, e.g. Chen and Tang [30] on isoparametric quasi-conforming fi- nite element, Jiang [74] on a higher precision triangular plate element and Tang et al. [151] on a multi-variable quasi- conforming quadrilateral element. The early elements are, naturally, linear formulations.}

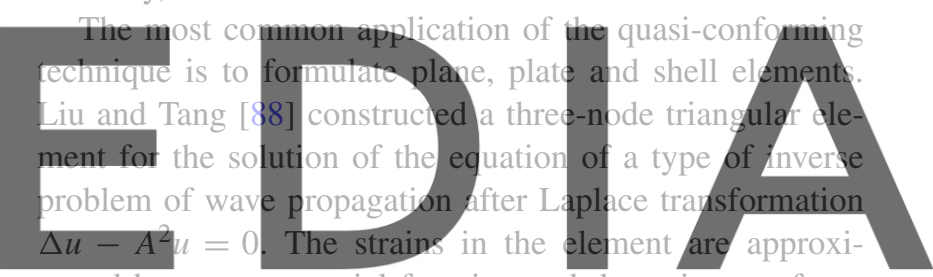

mated by an exponential function and the string-net function between neighboring elements is approximated by onedownlpad the version dwitheut the wwatermark

showed high precision for with course meshes. On plate elements, Zhao and Chen [185] presented a new approach to formulate thin plate bending elements. The strain energy of the element is decomposed into two parts: an integral concerning the first strain invariant and a line integral around the elemental boundary. The former can be discretized by quasi-conforming technique and the latter can be directly calculated using the interpolation of the deflection and its normal slope along the element boundary.

It is also possible to formulate axisymmetric quasiconforming elements. Guan and Zhang [56] constructed an axisymmetric three node degenerated shell element by combining the quasi-conforming FEM with the concept of the degenerated shell element. The axisymmetric three-node quasi-conforming degenerated shell element is formulated with the Hu-Washizu generalized variational principle. The stress and the strain are assumed by using the same isoparametric coordinate interpolation. To define the interpolation forms of the stress and the strain, numerical trials and analysis for eigenvalues of the element stiffness matrix were carried out. 
The present author have also contributed to quasi-conforming shell element literature with Kim et al. [81]. Two shell elements with drilling degrees of freedom, which improve membrane behavior and allows the modeling of stiffened plates and shells, were presented. The stiffness matrices are explicitly expressed, and the stresses are accurately obtained at the nodes.

The earliest literature in nonlinear quasi-conforming is that of Jiang [75]. The next one is by Guan and Tang [54], which also deals with geometric nonlinearity. In 1992, Guan and Tang [53] initially developed a linear nine-node quasiconforming degenerate shell element, based on the HuWashizu variational principle. This shell element is completely free from shear and membrane locking phenomena, and spurious kinematic modes were globally suppressed. Thereafter, Guan and Tang [55] constructed a geometrically non-linear quasi-conforming nine-node quadrilateral degenerated solid shell element based upon the non-linear quasiconforming finite element method. The linear and nonlinear strains are approximated with the same assumed strain and weighing function. Various nonlinear benchmarks were solved to verify the shell formulation. It was noted that the results indicate that the shell element in the paper were accurate and efficient, however, the results did not show

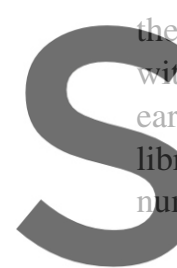
snapback and snap-through these benchmark problen ar clamped plate also shows librum path. In both the linear and nonli merical integrations were used. Qin [112] along with presentin
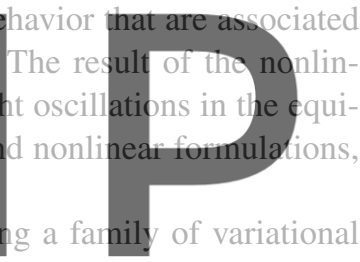
principles developed an eight-node isoparametric quasiconforming element, also geometrically. The element has

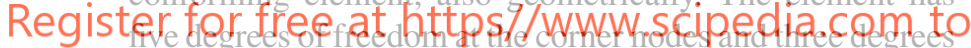
of freedom at the mid-side nodes. The examples presented include only geometric stiffening and not the complete postbuckling behaviour associated with the problems solved.

The works that are the main influence for this research are those by Voyiadjis and Shi. Voyiadjis and Shi conducted several studies on quasi-conforming plates and shells. In 1991, Shi and Voyiadjis [132-134] proposed a refined two dimensional shell theory for the thick cylindrical shells and developed a simple and efficient $\mathrm{C} 0$ cylindrical quadrilateral shell element using the quasi-conforming technique. Shi and Voyiadjis [133] have also developed the QCCP2 plate bending elements using the quasiconforming technique. By using an interrelated displacement-rotation function, the plate element was capable of automatically satisfying the Kirchhoff assumption for the case of thin plates. Shi and Voyiadjis [135] have extended their work by presenting the non-layered elasto-plastic formulation of shear deformable plates. The on-layered plasticity is based on a plastic hinge formulation and the modified Ilyushin's yield function that accounts for the development of plastic deformations across the thickness and the effects of transverse shear forces. After this, they continued their work by presenting the geometrically nonlinear analysis on plates and shells in Shi and Voyiadjis [133] and Voyiadjis and Shi [161]. Their formulation involves large displacements but small strains and a von Karman assumption. Large displacements meant large translations and rigid rotations but moderately large relative rotations. The plate and shell elements have five local degrees of freedom per node, three translations and two rotations. What should also be emphasized in their works is that their plate and shell formulations are given explicitly, whether linear or nonlinear.

Of late, Voyiadjis have continued to research on plasticity using quasi-conforming elements. Voyiadjis and Woelke [162] and Woelke et al. [173] presented studies on elastoplasticity of shells, where the shells were formulated using the quasi-conforming technique. To incorporate the formulated elasto-plastic theories into the shell element formulation, a plastic node concept was used. The plasticity is concentrated at the nodes, the nodes become plastic while the interior of the element remains elastic. The geometric nonlinearity is based on the von Karman assumption. The first article discusses thick shells, while the second has emphasis on damage due to micro-voids for thin and thick shells.

Another elastic-plastic quasi-conforming shell finite el-

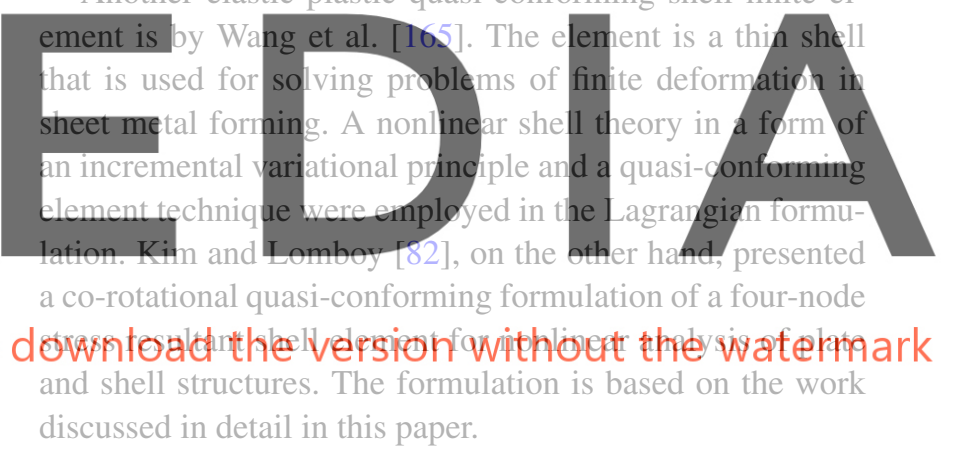

Searching for literature on laminate composite elements formulated using the quasi-conforming technique, three formulations had been found. The first is by Zhu and Wang [186] on a quasi-conforming penalty FEM for large deflection of composite laminated plate, in Chinese. The second is the one published by Park et al. [104], a quasiconforming formulation of a 4-node stress resultant shell element for linear static and dynamic analysis of composite plates and shells. The element and its formulation is the four-node quasi-conforming laminate composite in this paper and the static case results are those reported in Lomboy [89]. The third is that by Huang et al. [65]. They presented a quasi-conforming triangular laminated shell element based on a refined first-order shear deformation theory. The quasiconforming element is derived via the $\mathrm{Hu}$-Washizu variational principle with the stresses as Lagrange multipliers. The shell element has 7 degrees of freedom per node; three displacements, two first derivatives in the in-plane directions of the out-of-plane displacement, and two transverse shear 
strains. To consider the effect of transverse shear deformation on the global behavior of the laminated composite shell, the Reissner-Mindlin first-order theory, with shear correction factors of Whitney [169], was adopted. The formulation includes geometric nonlinearity and a von Karman assumption is used. The constant strain field is assumed for the midsurface linear and nonlinear strains. In a later study, Huang and Atluri [64] used the quasi-conforming shear flexible triangular element in developing a simple method to follow post-buckling paths in finite element analysis.

\subsection{Mathematical Studies on the Quasi-Conforming Technique}

The quasi-conforming technique properties and elements have also been studied on a purely mathematical background. Zhang and Wang $[181,183,184]$ studied the quasi conforming element by using a generalized patch test and tried to establish here the mathematical foundation of quasiconforming element for plate bending elements. In 1986, they also discussed the eonvergence of the quasi-conforming method. They showed that the quasi-conforming element method is convergent if the quasi-conforming element spaces have the approximability and the strong continuity.

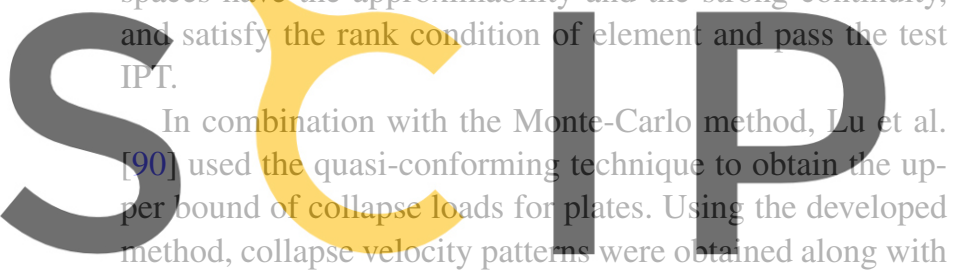

the collapse load of arbitrarily shaped plates.

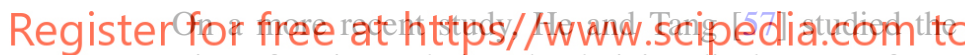
quasi-conforming technique by deriving displacement functions from the strain formulation constructed using the quasi-conforming technique. Using this method, it had been concluded that the coefficients of the constant and the linear strain are uniquely determined, and the quasi-conforming finite element method is convergent to constant strain. Also, different methods to determine the rigid displacement correspond to different displacement function and node error.

\subsection{Shell Finite Elements}

The formulation of shell elements is one of the most challenging tasks in finite elements. Its complicating factors include large displacements and rotations in 3D space, shear and membrane locking and material nonlinearity. In trying to solve these problems, an enormous amount of literature is now available on shell element formulations. In the works of Kanok-Nukulchai [77], the strategies of shell element formulation had been grouped into a) 3D continuum, b) classical shell and c) degenerate shell. Ma [91] added a 'superposed elements' classification from the latter.
The 3D continuum elements are formulated using threedimensional field equations. The resulting elements, without modification, gives poor performance when used with one dimension much smaller the others. To improve the element's performance, Wilson et al. [172] recommended the use of incompatible modes. The classical shell category includes shell elements formulated by reducing the threedimensional field equations with shell assumptions and the Kirchhoff-Love hypothesis. The degenerated approach is the largest category and has dominated shell finite elements since it was introduced in Ahmad et al. [2]. The 3D field equations are discretized in terms of mid-surface nodal variables and the formulation also includes the transverse shear effect. Its main drawback was the presence of shear and membrane locking. The alleviation and removal of these locking phenomena had been a focus of researchers for years and is still considered in the evaluation of good shell elements.

Aside from literature reviews included in individual journal articles, journal articles dealing only with advances in shell element formulations have also been published, Yang et al. [177, 179]. Yang and co-workers' review have focused on recent shell finite element formulations. Their classification of element formulations are a) degenerate shell, b)

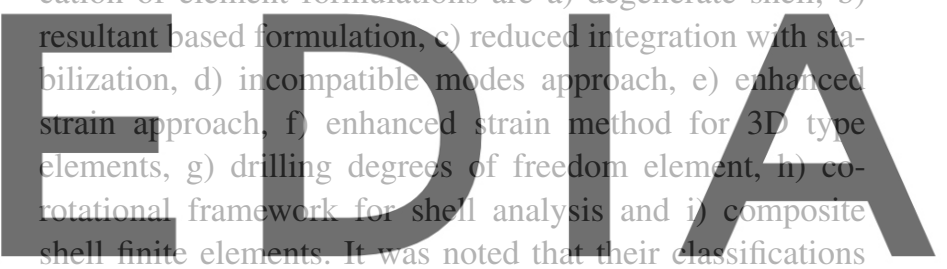

are not rigid and that recent successful shell finite elements

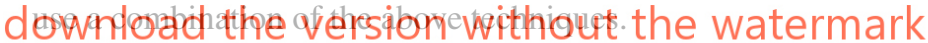

The resultant shell formulation is based on replacing the general set of three-dimensional governing equations with equivalent equations by pre-integrating or analytically integrating through the thickness of the shell. The preintegration gives an insight into the structure of the governing equations and leads to the formulation of effective finite elements. One of the first works in resultant shell formulation in the context of the 'natural approach' is by Argyris et al. [4-7]. They have formulated the SHEBA family of shell elements using the classical matrix displacement method with high order interpolations. Another significant resultant shell formulation that has been followed and modified by other researchers is by Simo et al. [136-144]. Their work proposed a stress-resultant-based geometrically exact shell model that is essentially equivalent to a one director inextensible Cosserat surface.

Reduced integration was originally proposed to treat locking problems. Yang's classification of reduced integration with stabilization focuses on the use of reduced integration to develop simple, efficient and robust elements. Simple and efficient in mesh generation and computing and robust 
in solving complicated non-linear problems with multiple contacting surfaces. Elements derived using purely reduced integration techniques prove to be rank deficient. In some cases, the rank deficiency resulted in singularities and oscillations or spurious modes. The use of stabilization matrices is one of the solutions that have been utilized to maintain a computationally efficient element while controlling the spurious modes. The ultimate reduced integration is the use of only one integration-point. Belytschko and co-workers [15, 17-21] extensively studied the development of a four-node quadrilateral using one integration point with stabilization. Belytschko and Tsay [19] quadrilateral element used one integration point for linear analysis and a column of integration points for material nonlinear analysis. Its main drawback is its failure to solve problems with warped elements. This element is widely used in the highly nonlinear crashworthiness studies. Liu et al. [87] presented a degenerated non-linear shell element using stress resultants. The shell element has an explicit tangent stiffness and is rank sufficient. The stabilization employed is based on a Taylor series expansion.

Incompatible modes have been introduced to overcome overly stiff elements and incompressibility. Wilson et al. [172] introduced the method of incompatible modes. It was

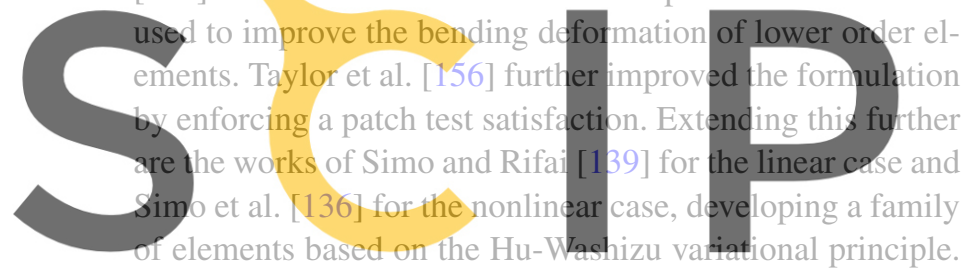

The elements perform well in bending, as well as for incom-

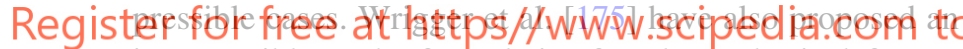
incompatible modes formulation for a large elastic deformation element.

In the work of Simo et al. [136], the enhanced strain approach was made developed. Instead of incompatible modes, the enhanced strain field is constructed directly. It incorporates the method of incompatible modes as a particular case. Elements having enhanced strains show very good coarse mesh accuracy. Examples of enhanced strain elements include formulations by Reddy and Wriggers [66], Basar et al. [10], Crisfield et al. [32] and Ish and Guttal [72]. The enhanced strain method has also been applied to 3D type elements, sometimes referred to as solid shells. Solid shells with enhanced strains have been formulated via stabilization methods, hybrid or mixed variational principles and mixed $\mathrm{Hu}$-Washizu variational principles.

The addition of drilling degrees of freedom allows convenient analysis of folded and stiffened plates and shells. The easiest way to incorporate a drilling stiffness is by adding a fictitious spring. The most commons is by using a strainenergy for the rotation of the shell normal, as introduced by Kanok-Nukulchai [77]. Hughes et al. [67, 69, 70] had extensively studied drilling degrees of freedom. They have also presented a variational formulation for elasto-dynamics with drilling degrees of freedom. Allman [3] presented the 'vertex connectors', one of the most successful formulations on drilling degrees. Its derivation is simple and the results from the formulations are fairly accurate. Hughes and Brezzi [69] investigated variational principles employing independent rotation fields and proposed several formulations. They successfully developed modified principles of mixed (i.e. Reissner and Hu-Washizu) and displacement type and proved convergence for a variety of convenient interpolatory patterns. Hence, they argued that convenient interpolatory patterns require modifications of the classical variational framework. Simo [142] and Ibrahimbegovic et al. [71] presented drilling rotations in a stress-resultant-based geometrically nonlinear shell model.

Traditionally, the implementation of most large rotation finite element formulations has been carried out in a single module. The constitutive law and the element kinematic descriptions are tightly coupled. This approach renders many existing beam and shell finite elements, based on moderate rotation assumptions, ineffective for large rotation problems. Belytschko and Hsieh [16] proposed a method based on convected coordinates to develop a small strain, large rotation

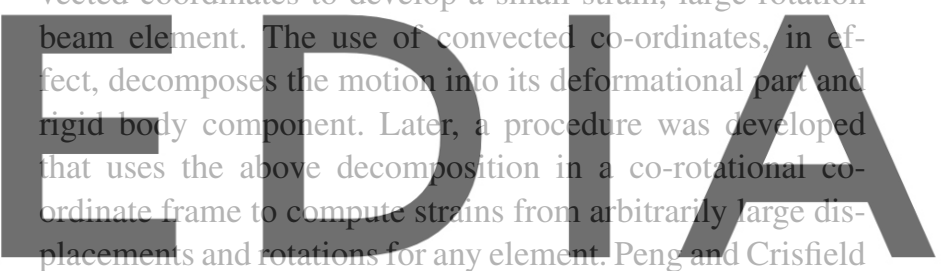

[106] described an alternate approach that involves a form

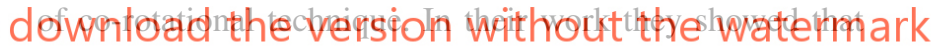
once the co-rotational technique is extended to large-strain plasticity, there are some advantages in considering the corotational framework. Moita and Crisfield [94] developed enhanced lower-order element formulations for large strains where they showed that a more general procedure could be devised with the aid of mixed assumed strain procedures. While Gruttman et al. [52] and Wriggers and Gruttmann [174] developed thin shell formulation with finite rotations based on the concept of Biot stress. Recently, Felippa and Haugen [48] presented a unified theoretical framework for the element independent co-rotational formulation in geometrical nonlinear analysis.

Laminated composite materials have often been modelled as an equivalent single layer using classical laminate theory (CLT), in which the thickness stress components are ignored and the Kirchhoff-Love kinematic hypothesis is enforced. However, laminates are susceptible to thickness effects because their effective transverse modulii are significantly small compared to the effective elastic modulus along the fibre direction. As a result CLT under-predicts deflections and over predicts natural frequencies and buckling 
loads. Refined single layer theories have been proposed to overcome these deficiencies, Kapania and Raciti [79]. Whitney and Pagano [170] first developed a Mindlin-type firstorder transverse shear deformation theory (FSDT) for multilayered anisotropic plates, and Dong and Tso [40] for multilayered anisotropic shells. Modelling laminates with single layer theories cannot account for cross-section warping, results in incompatible shearing stresses between layers and are inadequate for accurate local stress analysis. A higherorder theory overcoming some of these limitations was presented by Reddy [116] for laminated plates and by Reddy and Liu [119] for laminated shells.

Variationally sound theories that accounts for the 3-D effects, allows thickness variation, and permits the warping of the deformed normal for a refined analysis of thick and thin composites have also been proposed. Two classes of theories are available that can, short of a full-fledged three-dimensional analysis of plates and shells, accurately and efficiently predict the stress distribution: layerwise theories and the individual layer plate theory. In the layerwise plate theory, suggested by Reddy [115], the continuity of the transverse normal and shear stresses is not enforced. In the individual-layer plate models, for example Di Sciuva [37], the transverse shear stress continuity is enforced a priori. A

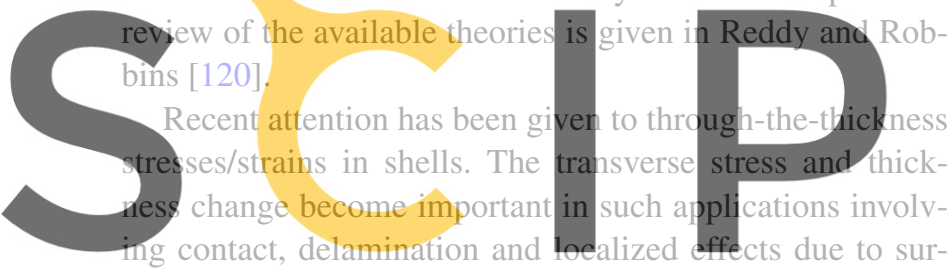
face loading. Accounting for thickness deformation is also

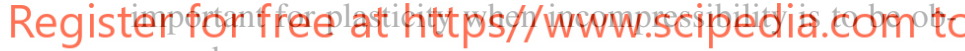
served.

Basar and Ding [9] presented the theoretical and numerical simulation of large strain rubber-like shells using shear deformable models. The shell model includes two thicknessstretching parameters in a quadratic displacement function. Their formulation applies to compressible and incompressible materials. Betsch and Gruttmann [22] formulated a four-node shell using three-dimensional stress/strain and hyperelastic material laws. No shell specific approximations of the three dimensional constitutive equations are incorporated. The discretization of the covariant Green-Lagrange strains leads directly to the interpolation of the thickness stretch. Accordingly, the thickness stretch is included within the interpolation constants of the generalized finite element displacement vector. El-Abbasi and Meguid [45] developed a seven-parameter model. Two interpolation schemes for the shell director were developed to avoid thickness locking. The formulation was test for both thin and thick shells. Brank et al. [24] also tackled shell thickness deformation. They studied three different formulation of incorporating through-the-thickness stretching, a seven-parameter model, a model that uses an additive decomposition of the strains and the other an additive decomposition of the deformation gradient. In a more recent research, Tan and Vu-Quoc [148] presented a low-order solid-shell element formulation for analyses of large deformable multilayer shell structures with non-linear materials. The locking associated with nearly incompressible materials was avoided by the enhanced assumed strain method (EAS). Shear locking and curvature thickness locking are resolved effectively by using the assumed natural strain (ANS) method.

\subsection{Shear and Membrane Locking}

The degenerated approach has dominated shell finite elements since it was introduced in Ahmad et al. [2]. Early formulations using the degenerate concept on shear flexible elements, however, sometimes suffer from two types of deficiencies. The two deficiencies are shear locking and membrane locking. While such elements are capable of dealing with thick plates and shell problems, their performance may deteriorate rapidly as the thickness becomes smaller. Shear locking may possibly occur in both flat and curved shear deformable bending elements, while membrane locking occurs only in curved thin shell.

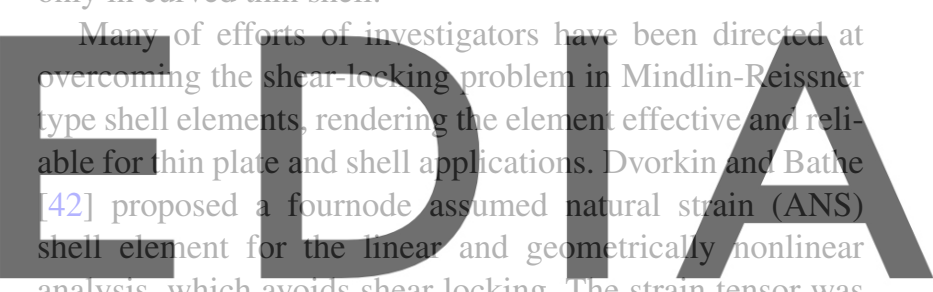

analysis, which avoids shear locking. The strain tensor was expressed in terms of the covariant components and covaridownadoad the hersiphand wout thenwatermark

satisfactory and suggested promising results in very complex shell structures. Dvorkin et al. [43] presented a finite strain elasto-plastic formulation of MITC4. Simo and Rifai [139] developed a class of enhanced assumed strain (EAS) element formulation using a three-field mixed finite element framework. The assumed strain method does not only solve the locking problems, but also improves the elements accuracy for coarse meshes and performs very well in the incompressible limit. Combining the EAS and the assumed natural strain (ANS) in an efficient manner, Vu-Quoc and Tan [163, 164] presented a solid-shell element formulation. In their work, a modified EAS is used to improve the element performance and pass the patch test, while ANS is used to remove shear locking and membrane locking.

Another method, which has been adopted to solve locking problems, is the reduced integration or selective integration technique. Some of the early uses of the reduced/selective integration scheme were by Doherty et al. [39], Zienkiewicz et al. [187] and Pawsey and Clough [105]. One of the researches that have explained the locking behaviour and its alleviation by selective integration, through 
a heuristic argument, was by Hughes et al. [68]. Their work was applied to a plate element. This was then followed by the application of the same type of integration to a 4-node shell element by Kanok-Nukulchai [77]. Selective integration is a solution for shear locking problems; however, the application of under-integration has been shown to result in the appearance of spurious zero-energy modes. The spurious modes are due to rank deficiency resulting from underintegration. A remedy to the rank deficiency, which is widely used today, is the use of a stabilization matrix.

\subsection{Laminate Composite Progressive Failure}

Fibrous composite is a good material for structures because of low weight, high stiffness, high strength, longer fatigue life, and optimal or tailored design. Most of its applications have been in aerospace engineering and automotive design. Recently, composite materials are being used as bridge decks and concrete reinforcement and repair.

Like most design methods, the ability to predict the behaviour of a structure during its stages of near failure to final failure is important. Fibrous composite is not an exception. To be able to predict its response during first layer damage and last layer damage would give designers the idea how

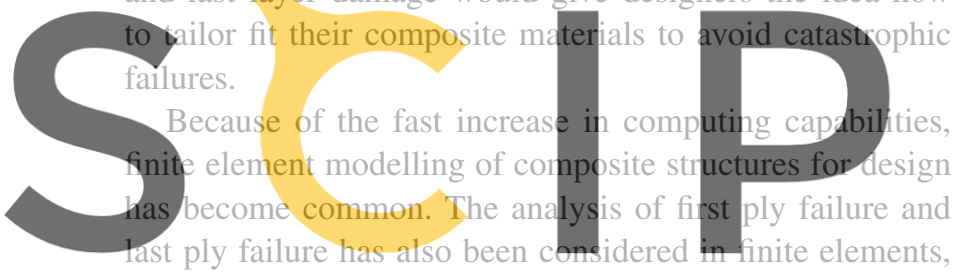
such as in the works of Engelstad et al. [47], Sandhu et al.

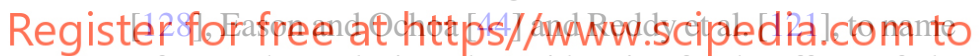
a few. In the analysis and consideration for the effects of ply failure, present methods considers the stresses at the gauss points and weighs the effects of damage by using the weight of the Gauss point.

Different authors had studied progressive damage using macroscopic failure theories in finite elements extensively. Ochoa and Engblom [99] used a higher-order displacement element. The element had eight nodes with seven degrees of freedom for the corner nodes and three degrees of freedom for the side nodes. Toson and Zabaras [158] studied several failure criteria using finite element method. The maximum stress, Hoffman, Tsai-Wu, Lee and Hashin failure criteria were considered. There results showed that the first ply failure and last ply failure using the Lee criterion gave the best results. Engelstad et al. [47] used a nine-node quadratic shell element based on a continuum formulation of a laminated 3$\mathrm{D}$ shell element to predict the post buckling behaviour and progressive failure of composite panels in compression. The failure in each layer was checked at the Gauss points of each layer. When failure occurred, laminate properties were reduced at the Gauss point.
In more recent works, Banbury et al. [8] used the maximum principal strain criterion to predict the progressive failure of fastener pull-trough. Very good correlation between experimental results and predicted pull-through failure loads, failure location and failure sequence were achieved. Padhi et al. [103] studied the progressive failure and ultimate collapse of laminated composite plates subject to transverse pressure. Several failure criteria were used including Hashin's and Tsai-Wu's and the stiffness reduction resulting from ply failure was evaluated at the gauss points. Spottswood and Palazotto [146] studied the physical response of a curved panel under transverse loading. Their study showed that the physical response of the panel would predict a more realistic response with the inclusion of progressive failure.

One of the early works on non-linear transient analysis of composites were by Reddy [114] and Reddy and Chandrashekhara [118]. Other notable works on the subject were those of Wu and Yang [176] and Kant and Mallikarjuna [78]. Transient analyses with damage of composites in recent studies are mostly focused on impact response, such as works by Wiggenraand et al. [171] and Geubelle and Baylor [50].

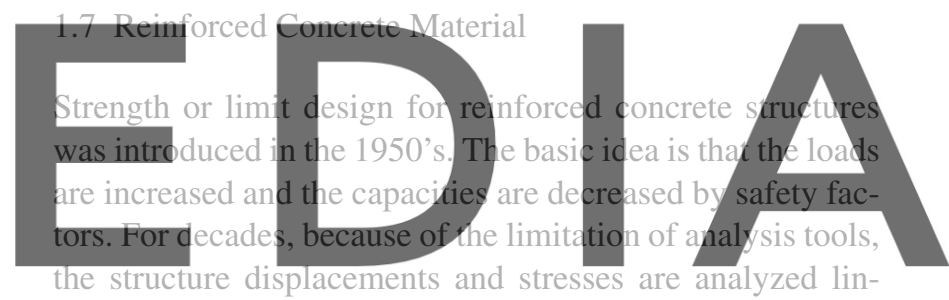

early, while the desion is conducted assuming nonlinear ma-

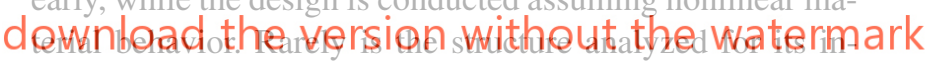

cremental nonlinear response after this point. However, research on finite element analysis of reinforced concrete that considers nonlinear materials and cracked cross-sections go back to the 1960's and 70's, [14, 26, 76, 85, 96, 129, 130]. But since the use of finite elements as an analysis method and design tool has been heavily dependent on the power and availability of computers, these were slow to seep into the workplace.

Concrete plate and shell structures vary from floor slabs, tanks, and roofs to large cooling towers, hence, their nonlinear analysis have been given much attention. With the advent of the performance based design method, analysis of nonlinear reinforced concrete shells that includes large deformations and material failure is becoming more important and with the growth of the power of the computer, more common. In the nonlinear analysis of reinforced concrete plates and shells, elements and constitutive models development go hand-in-hand. There are two methods by which reinforced concrete may be modeled: discrete and layered modeling. In discrete models, concrete is modeled by threedimensional solid elements while the reinforcing steel is 
modeled by truss elements. This method is computationally expensive because of the number of elements required to model a simple shell structure. Because of its simplicity in formulation, the degenerate approach combined with a layered model is much more widely used. Scordelis and Chan [131], Owen and Figueiras [102] and Polak and Vecchio $[109,110]$ are some examples that present formulations based on a layer model applied to degenerate shell element.

Concrete is a non-homogeneous and anisotropic material that behaves nonlinearly. The use of an experimental uniaxial stress-strain curve for constitutive modeling to concrete is insufficient to describe the strength characteristics of concrete under multi-axial stress states. Its compressive strength increases as it is loaded in a biaxial compressive state and decreases as the tensile stress is increased under biaxial compression-tension. Various constitutive models and analysis procedures have been presented on this account [12, $13,34,35,46,63,86,93,100,101,160]$. These formulations have been shown to provide reasonably accurate simulation of response under a variety of complex structural conditions and loading schemes.

This study was conducted to present an efficient formulation of a material and a geometrically nonlinear shell element using the quasi-conforming techniques. To han-

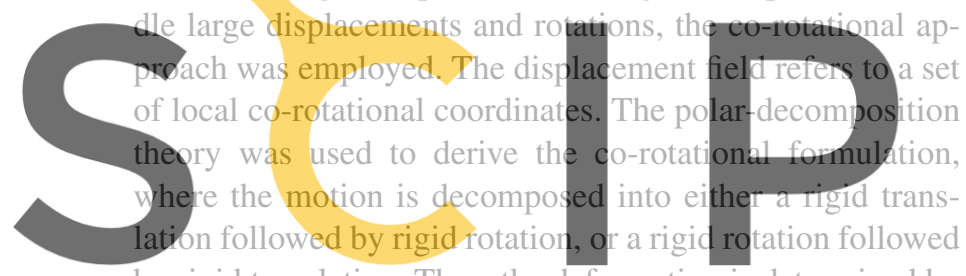
by rigid translation. Thus, the deformation is determined by removing the rigid-body rotation from the total nodal dis-

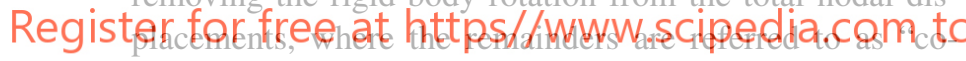
rotational displacements."

To improve the membrane behavior and the modelstiffened plate and shell structures, the rotational degree of freedom and rotational strain of the normal shell was included in both the linear and nonlinear quasi-conforming formulation. Instead of the von Karman assumption [135], the present geometrically nonlinear formulation was derived using the full definition of the Green strain tensor, which includes the membrane, bending, and transverse shear stresses in the geometric stiffness.

Compared with the other Lagrangian element, the stresses in the present formulation directly occur at the nodes instead of the usual Gauss integration points. The element matrices are explicitly integrated (not numerically), making the element computationally efficient and less sensitive to distortion, which are desirable for nonlinear analysis. Moreover, since the stresses occur at the nodes, the onsets of the maximum stresses are obtained accurately, as opposed to those taken within the interior of the element or Gauss points.

The formulations of the nonlinear material are present based on the layer concept, and the material properties can vary throughout the thickness and across the surface of a shell element. The stress stations are equally spaced through the thickness, two of which are at the top and bottom surfaces, making up the so-called "multilayered approach." The actual stress components are assumed to be the plane stress components at any level of thickness. A modified trapezoidal rule is used for the numerical integration of the constitutive relation in the plasticity part. Thus, the present shell element is very efficient in capturing the nonlinear behavior of a material.

For elastoplastic analysis, the von Mises yield condition with linear strain-hardening and the Prandtl-Reuss flow rule to discrete points, through the thickness, was used. For the analysis of a laminate composite material, the various failure criteria for laminate composite materials were included in the formulation, which made it possible to analyze the progressive failure of the fiber and the matrix.

The material nonlinearities that were included in the formulation of the reinforced concrete material were the cracking of the concrete under tension, the nonlinear response of the concrete in compression, and the yielding of the reinforcement. For the concrete in compression, the formulation was based on two different approaches: the plasticitybased model (perfect plasticity and strain hardening) [61]
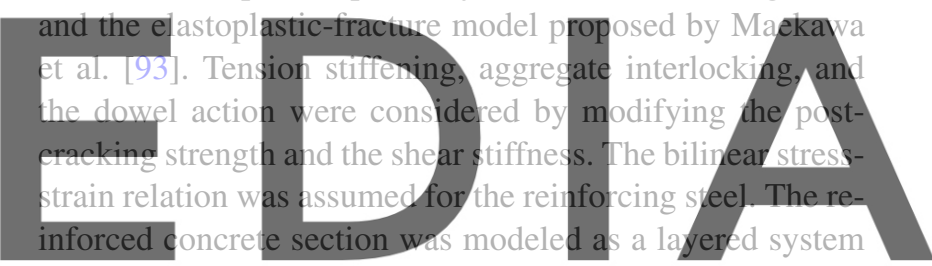

of concrete and of the equivalent smeared steel layer, assuming a perfect bond between the concrete and the steel downlapd the version withpHt the watermark several tests of linear, geometric, and nonlinear material problems were performed, and their results were compared with the data in the literature through the analytical and experimental procedures. The good performance of the proposed shell element was also shown in the numerical examples presented.

\section{Formulation of the Quasi-Conforming Technique}

\subsection{Basic Concept}

The quasi-conforming technique was introduced by Tang et al. [149, 153]. It was presented as a general method that treats the conforming, non-conforming, and hybrid elements in a unified way. This method was initially inspired by Pian's work [108] on the assumed stress formulation. Tang et al.'s work however, had emphasis on the interpolation functions for the improvement of finite elements.

Piecewise polynomial functions with variational constraint are commonly used to approximate solutions of the 


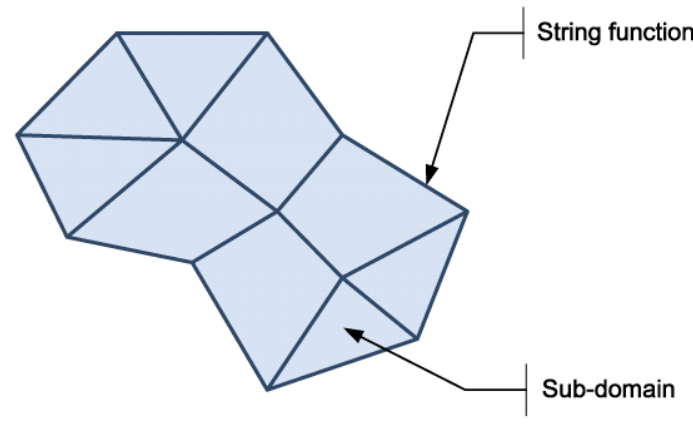

Fig. 1 Finite element mesh

finite element method. However, for cases where second order derivatives are involved, inter-element compatibility problems may arise when using piecewise polynomial functions. It would be very difficult, if not impossible, to describe smooth surfaces of prescribed curvature by a series of elements approximated with piecewise polynomials. In the quasi conforming technique, the surface is described using one dimensional interpolation functions, coined 'string functions', by forming, a 'net' within the surface. The one dimensional interpolation functions are used to obtain the strain discretization in the element by boundary integration.

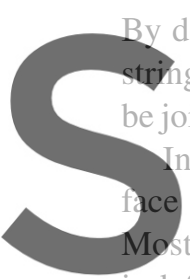
By doing this, when elements tring functions along the bounda be joined together and would form face is defined; be divided into is defined within each the sub-domain or element with piecewise polynomial functions. The compatibility of the inter-

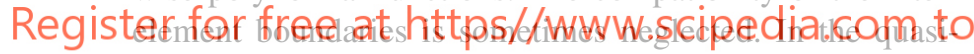
conforming technique, the functions describing the surface are defined only at the inter-element boundaries of the subdomains, and the functions inside the element are not defined explicitly.

The first step in formulating quasi-conforming finite elements is the discretization of the strains (surface/displacement derivatives) in an element (sub-domain). The strains may be

$\mathbf{e}=\left[\begin{array}{lllll}\frac{\partial u}{\partial r} & \frac{\partial u}{\partial s} & \frac{\partial^{2} u}{\partial r^{2}} & \frac{\partial^{2} u}{\partial s^{2}} & 2 \frac{\partial^{2} u}{\partial r \partial s}\end{array}\right]^{\mathrm{T}}$,

depending on the problem to be solved. The stains are assumed to be continuous within the element but discontinuous across the inter-element boundaries, and may be approximated by Taylor's expansion. For the first term of (1),

$$
\begin{aligned}
\frac{\partial u}{\partial r} \approx \tilde{e}= & {\left[\frac{\partial u}{\partial r}\right]_{0}+\left[\frac{\partial^{2} u}{\partial r^{2}}\right]_{0} r+\left[\frac{\partial^{2} u}{\partial r \partial s}\right]_{0} s } \\
& +\left[\frac{\partial^{3} u}{\partial r^{3}}\right]_{0} \frac{r^{2}}{2}+\cdots
\end{aligned}
$$

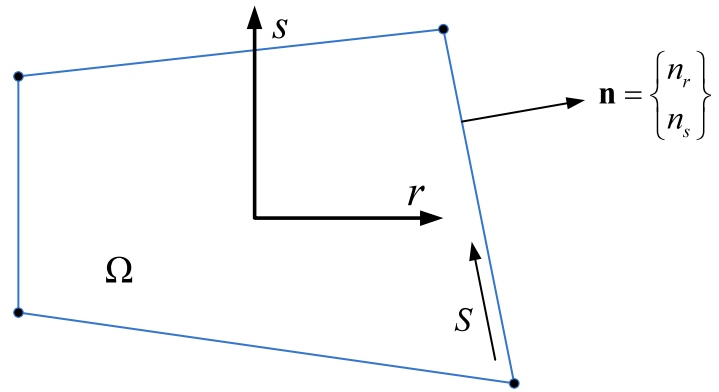

Fig. 2 Boundary line $S$, domain $\Omega$ and boundary normal $n$

The $\sim$ is to denote, the expansion approximate. Equation (2) can also be expressed as a truncated polynomial expansion,

$$
\begin{aligned}
\frac{\partial u}{\partial r} \approx \tilde{e} & =\alpha_{1}+\alpha_{2} r+\alpha_{3} s+\alpha_{4} r^{2}+\cdots \\
& =\sum_{i=1}^{n} P_{i} \alpha_{i}=\mathbb{P} \alpha
\end{aligned}
$$

The $P_{i}$ 's are the basis trial functions. Enforcing the above approximation within the element,
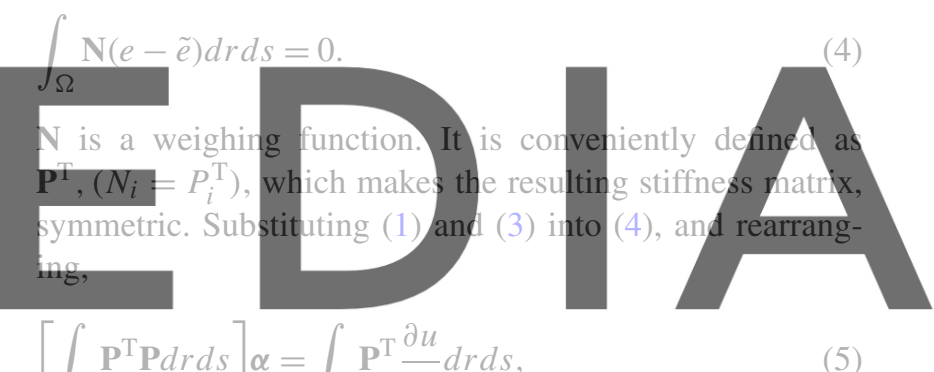

download the versión without the watermark $\mathrm{A} \alpha=\mathrm{Cu}$,

$\mathbf{A}=\int_{\Omega} \mathbf{P}^{\mathrm{T}} \mathbf{P} d r d s \quad$ and $\quad \mathbf{C u}=\int_{\Omega} \mathbf{P}^{\mathrm{T}} \frac{\partial u}{\partial r} d r d s$.

The integral on the left side of (5) are composed of polynomials, and may be integrated easily. Using Green's theorem, the right hand side integral can be evaluated.

$$
\int_{\Omega} \mathbf{P}^{\mathrm{T}} \frac{\partial u}{\partial r} d r d s=\oint_{S} u \mathbf{P}^{\mathrm{T}} n_{r} d S-\int_{\Omega} u \frac{\partial \mathbf{P}^{\mathrm{T}}}{\partial r} d r d s .
$$

$S$ is the boundary line of the element, $n_{r}=\cos (n, r)$ and $n$ is the outward normal of the element boundary, Fig. 2. For the case of second order derivatives,

$$
\begin{aligned}
\int_{\Omega} \frac{\partial^{2} u}{\partial r \partial s} \mathbf{P}^{\mathrm{T}} d r d s= & \frac{1}{2}\left\{\oint_{S}\left(\frac{\partial u}{\partial s} n_{r}+\frac{\partial u}{\partial r} n_{s}\right) \mathbf{P}^{\mathrm{T}} d S\right. \\
& -\oint_{S}\left(\frac{\partial \mathbf{P}^{\mathrm{T}}}{\partial s} n_{r}+\frac{\partial \mathbf{P}^{\mathrm{T}}}{\partial r} n_{s}\right) u d S \\
& \left.+2 \int_{\Omega} u \frac{\partial^{2} \mathbf{P}^{\mathrm{T}}}{\partial r \partial s} d r d s\right\} .
\end{aligned}
$$


The line integrals in (8) and (9) are evaluated using string functions, introducing what has been earlier mentioned as string functions along the boundary of the element. Interelement compatibility is then achieved within the integral. When area integrals remain, such as the last terms in (8) and (9), they may be evaluated without concern for inter-element compatibility. When both the line integral and the area integral are present, the displacement could be said to be composed of compatible and incompatible modes. Both types of integration, the line integrals and area integral, should be integrated with sufficient accuracy and in terms of nodal displacement values.

Equations (3) to (9) is done to all strain terms to obtain the strain discretization matrix, B. Thus, the element strain field is,

$\mathrm{e}=\mathrm{PA}^{-1} \mathrm{Cu}=\mathrm{Bu}$.

A, $\mathbf{P}$ and $\mathbf{C}$ are defined to contain all the parameters for each strain term.

2.2 Element Displacements

In the quasi-conforming technique, the key part in defining the displacements is the element boundary displacements. The boundary displacements an ment functions used in the integ Imation, specifically the seconc fynction is called the string fy mbled, their boundaries fo Jacement function forms as orc cor In choosing/defining the sting functions, it is recommended

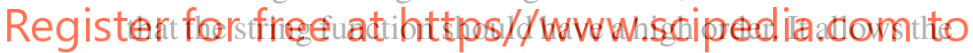
element to have a high order displacement function while remains easily integrated.

Since string functions are 1D displacement functions, it only defines the displacements of a reference line or plane. This poses no concern for plane elements but for the case of shells, string functions would refer only to displacements on mid-surface. This limits the application to degenerate formulations.

When area integrations remain in (8) and (9), a displacement function that describes the displacement within the element reference surface has to be defined. This may be done using isoparametric mapping. They may be evaluated without concern for inter-element compatibility.

The displacement relations for parts of the element that do not lie within the reference surface may be described using any kinematic relations. These will affect the quasiconforming formulation when the element strains are derived. Substitution of the kinematic relations to the definition of the strain terms, example Green Lagrange strains, will provide an expression of strains that will be approximated using the quasi-conforming technique.
In the nonlinear formulation of quasi-conforming elements, a co-rotational approach is adopted. This allows the analysis of problems involving large element rotations and translations. It is easy to separate rigid body rotations and translations from strain producing pure deformation. The assumption is that rotations and translations can be large, but strains remain small.

Since the element strains are interpolated in local coordinates via Taylor's expansion in (3), it is also convenient to express the element formulation in a local co-rotational coordinates.

The local coordinate origin is located at the geometric center of the element. It is assumed that this point is unchanged, relative to the element boundaries or nodes. Considering that the element would undergo large displacements, the local corotational axes should displace with the element while remaining independent of the element deformation or strains. This is achieved by, first assigning two lines of element fibers that are passing through the origin. These lines do not need to be orthogonal. The direction of these lines should be determinable in the undeformed and deformed states. The vectors along these lines would be $\mathbf{l}_{1}$ and $\mathbb{I}_{2}$. The local orthogonal surface axes can then be placed symmetrically with $\mathbb{I}_{1}$ and $\mathbb{I}_{2}$ so that even as the element, thus

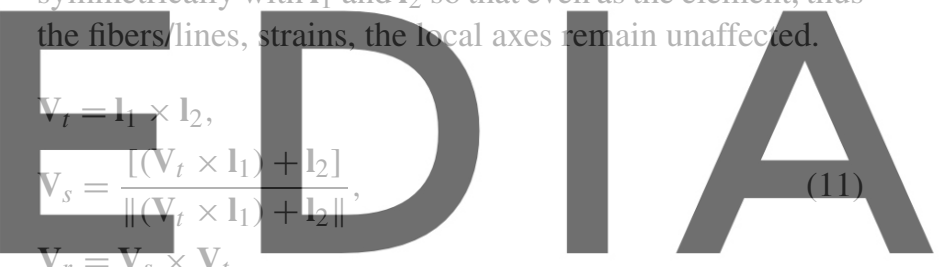

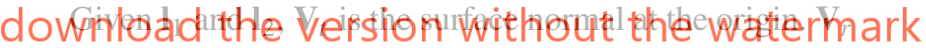 and $\mathbf{V}_{S}$ are the base vectors of axes $r$ and $s$ that lie on the reference surface. In (11), symmetric means angles $\mathbf{V}_{\mathbf{s}}-\mathbb{I}_{2}$ and $\mathbf{V}_{r}-\mathbf{l}_{1}$ are always equal, as shown in Fig. 3.}

\subsection{Element Strains}

The total strain at any point $p$ is given by the linear corotational strain tensor

$\varepsilon_{i j}=\frac{1}{2}\left(\hat{u}_{i, j}+\hat{u}_{j, i}\right)$.

The linear strain terms are expressed in terms of nodal displacements using the quasi-conforming technique. The strain function would take the form of (10). The first step in deriving (10) is to assign the terms of $\mathbf{P}$. The number of polynomial terms to express the distribution of strain over the element should be enough for the element to be rank sufficient. To determine whether the element is rank sufficient, the following equation should be satisfied

$M-K \geq N-G$. 
Fig. 3 Local co-rotational axes

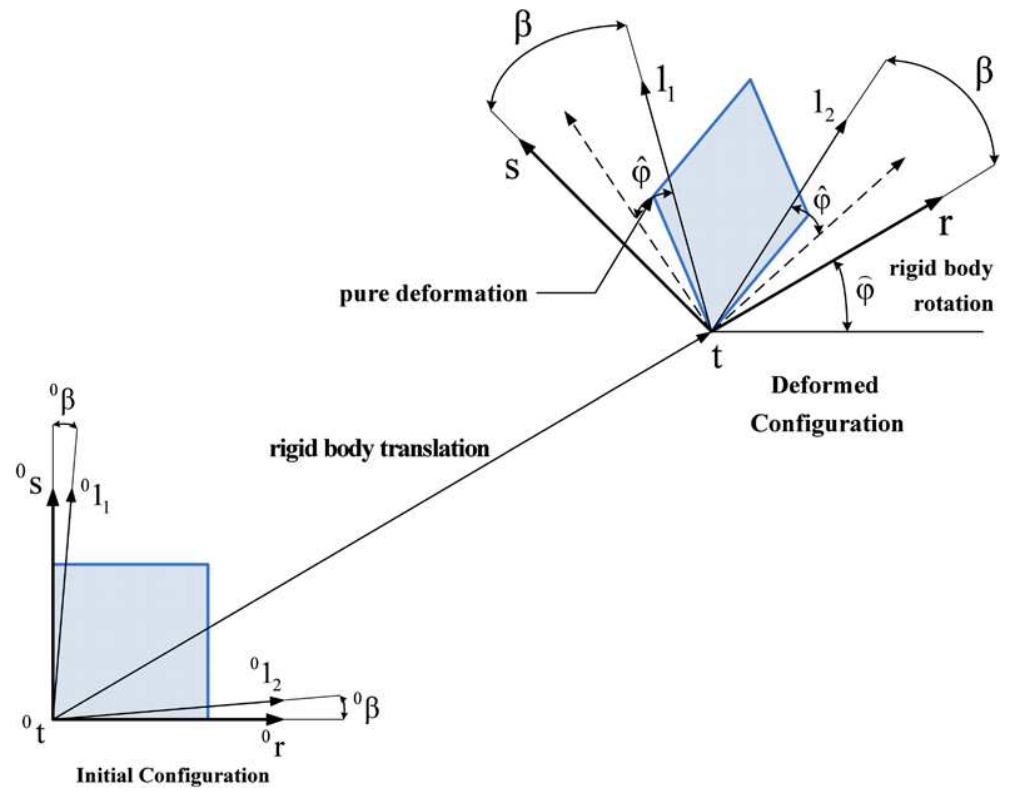

$M$ is the number of constants of strain and $K$ is the number of dependent constants of strain due to the differential compatibility relations, which are

$2 \frac{\partial}{\partial y}\left(\frac{\partial^{2} w}{\partial x^{2}}\right)=\frac{\partial}{\partial x}\left(2 \frac{\partial^{2} w}{\partial x \partial y}\right)$ and

$2 \frac{\partial}{\partial x}\left(\frac{\partial^{2} w}{\partial y^{2}}\right)=\frac{\partial}{\partial y}\left(2 \frac{\partial^{2} w}{\partial x \partial y}\right)$.

$N$ is the number of parameters, i.e. the degrees of freedom of displacements and $G$ is the number of rigid body displacements.

The next term to be evaluated is matrix $\mathbf{A}$, the first of (7). Because of its definition, it is a symmetric matrix composed of polynomials. For elements without midside nodes, A may be easily integrated. The integration formulas that could be used are

$$
\begin{aligned}
& \int_{\Omega} r^{m} s^{n} d r d s=\frac{1}{m+1} \oint r^{m+1} s^{n} n_{r} d S \quad \text { or } \\
& \int_{\Omega} r^{m} s^{n} d r d s=\frac{1}{n+1} \oint r^{m} s^{n+1} n_{s} d S .
\end{aligned}
$$

Elements with mid-side nodes would require coordinate transformation to be integrated, from local coordinates to natural coordinates.

The size and terms in $\mathbf{A}$ are dependent on the form of $\mathbf{P}$. When each strain term is approximated independently, i.e. each strain term has its own set of undetermined strain parameter $\boldsymbol{\alpha}, \mathbf{A}$ can be divided into sub-matrices and could simplify inversion. The four-node plane element in the following chapter has coupled strain terms, while the other element formulations have independent functions.
Matrix $\mathbf{C}$ is obtained from integration of the second equation in (7). The integration involves transformation of the integral into line integrals along the boundary. This transformation facilitates the use of string functions, the key to the quasi-conforming formulation. This part of the formulation is what requires most work.

\subsection{Stresses and Constitutive Relations}

The prediction of stresses is of major importance, more so in nonlinear analysis where the response is dependent on the element internal stresses. It is essential that the adopted stress measures are objective with respect to rigid body motion and that the stress and strain measures are work conjugate. For the present work, which adopts the updated Lagrangian form, the appropriate stress measure is the Cauchy stress tensor. In the co-rotational approach, the incremental Cauchy stresses are

$\Delta \sigma_{i j}=C_{i j k l} \Delta \varepsilon_{k l}$

$C_{i j k l}$ is the constitutive tensor. $\Delta \varepsilon_{k l}$ is the incremental form of (12), taken as the difference between the co-rotational strains in the current configuration and the co-rotational strains in the last equilibrium configuration.

In formulating the quasi-conforming elements, a stress resultant approach is adopted. Assuming small strain and constant thickness, the stresses is integrated though the elements cross-section. Stress resultants may be grouped into in-plane and transverse shear forces and moments.

$$
\begin{aligned}
S_{i j} & =\int_{\Omega} \sigma_{i j} d a \\
& =\int_{\Omega} C_{i j k l} \varepsilon_{k l} d a=D_{i j k l} \varepsilon_{k l},
\end{aligned}
$$


$S_{i j}$ is the stress resultant tensor. $\Omega$ represents the integration domain; cross section or thickness, as applicable. $D_{i j k l}$ is the rigidity matrix. In quasi-conforming and matrix form, the stress resultants take the form

$\mathbf{S}=\mathbf{D e ̂}$,

$\mathbf{S}=\mathbf{D P A}^{-1} \mathbf{C T}^{\mathrm{T}} \hat{\mathbf{U}}$.

For an elasto-plasticity, laminate composite with damage or concrete with cracks, $\mathbf{D}$ will not be constant throughout the element. Some region of the element may become plastic or become damaged first. Thus, to be able to form the element stiffness matrices and force vectors, an expression for $\mathbf{S}$ and D that considers different material conditions within the element has to be defined. For elements integrated numerically, this expression is not explicitly given. Correct integration is still possible as long as the order of Gauss integration is sufficient to that of the order of an actual $\mathbf{D}$ function.

As a result of performing explicit integration and use of boundary integration, implementation of the elasto-plastic formulation is not straightforward. An elasto-plastic formulation for the above would either be, to use plastic nodes similar to formulation of Voyiadjis and Shi [161] or to perform numerical integration. Here, the use of an explicit expression of the elasto-plastic stress field is explored.

Unlike elements integrated numerically, implementation of the above formulation will not have predefined stress points. Therefore, the first step will be to define stress points. The chosen stress points are the nodal points. For most finite element users, stresses of interest are at the nodal points. The user can exactly define the location of these points during the meshing stages. Thus, for both linear and non-linear analyses, stresses are directly taken from the nodal points. Since the nodal coordinates are known at any stage in the analysis, whether linear or incremental analysis, the stresses are easily obtained by substituting the current local nodal coordinates to (10) and computing for the corresponding stresses. Choosing the stress points as the nodal points eliminates extrapolation to the nodal points during post processing which is required for stresses taken from Gauss points. This is also convenient for models having initial stresses. Users do not have to compute for the initial stress at the element Gauss point or locate the Gauss point to the location of known stress.

Since stresses are a function of strains, the order of the stress function is the same as that of the strain. D also appears in the equation of motion from the expression of the stresses in terms of strains. A field expression for $\mathbf{D}$ should also therefore be of the same order as the stresses and strains. The explicit expressions for elasto-plastic $\mathbf{S}$ and D are formed using interpolation functions that should be consistent with the approximation of the strains.

$$
\begin{aligned}
& \mathbf{S}=\sum_{i=1}^{n} H_{i} \mathbf{S}_{i}, \\
& \mathbf{D}=\sum_{i=1}^{n} H_{i} \mathbf{D}_{i} .
\end{aligned}
$$

Where $n$ would depend on the number of stress points used and $\mathrm{H}_{i}$ 's is the interpolation function. For the material and geometric nonlinear formulation of the quasi-conforming technique, (20) is used to solve for the elasto-plastic stiffness matrix, while (19) is used to solve for the geometric stiffness and internal force vector.

\subsection{Equation of Motion}

In formulating the quasi-conforming elements, an updated Lagrangian formulation is adopted. The linearized equation of motion, is expresses as

$$
\begin{gathered}
\int_{{ }^{t} V}{ }^{t} C_{i j r s} \Delta e_{r s} \delta \Delta e_{i j} d{ }^{t} V+\int_{{ }^{t} V}{ }^{t} \sigma_{i j} \delta \Delta \Xi_{i j} d^{t} V \\
={ }^{t+\Delta t} \Re-\int_{{ }^{t} V}{ }^{t}{ }^{t}{ }_{i j} \delta \Delta e_{i j} d^{t} V .
\end{gathered}
$$

$C_{i j r s}$ is the component of the constitutive tensor, ${ }^{t} \sigma_{i j}$ is the component of the Cauchy stress tensor, ${ }^{t+\Delta t} \Re$ is the external virtual work expression, and $\Delta e_{i j}$ and $\Delta \Xi_{i j}$ are the incremental linear and nonlinear part of the Green's strain tensor, respectively. Assuming a small strains and a constant cross section during deformation, (21) may be expressed as

$$
\begin{gathered}
\int_{\Omega}\left(\delta \Delta \hat{\mathbf{e}}^{\mathrm{T}} \mathbf{D} \Delta \hat{\mathbf{e}}\right) d \Omega+\int_{\Omega}(\mathbf{S} \delta \Delta \hat{\mathbf{\Xi}}) d \Omega \\
={ }^{t+\Delta t} \Re-\int_{\Omega}\left(\delta \Delta \hat{\mathbf{e}}^{\mathrm{T}} \mathbf{S}\right) d \Omega .
\end{gathered}
$$

$\mathbf{D}$ is the rigidity matrix. $\mathbf{S}$ is the stress resultant vector. All the quantities are referred to the current configuration, except for ${ }^{t+\Delta t} \Re$, which is the external virtual work expression. The remaining integration is only along the length of the element. $\Delta \hat{\mathbf{e}}$ is the linear part of the Green's strain tensor and $\Delta \hat{\boldsymbol{\Xi}}$ is the nonlinear part.

\subsection{Stiffness Matrix due to Linear Strains}

The stiffness matrix due to linear strains is derived from the first term of the LHS of the linearized equation of motion. For the co-rotational resultant stress case

$\int_{\Omega}\left(\delta \Delta \hat{\mathbf{e}}^{\mathrm{T}} \mathbf{D} \Delta \hat{\mathbf{e}}\right) d \Omega$.

The incremental linear strains $\Delta \hat{\mathbf{e}}$ are expressed in terms of the nodal displacements using the quasi-conforming technique as discussed in Sect. 2.3. The rigidity matrix $\mathbf{D}$ is the 
stress-strain relation matrix that has been integrated about the element cross-section. For the present case, it is either elastic, elasto-plastic or laminate composite with layer failure.

Substituting the quasi-conforming expression of linear strains, (10), and the rigidity matrix $\mathbf{D},(20)$, into (23) gives the element stiffness matrix due to linear strains, $\mathbf{K}_{L}$.

$$
\begin{aligned}
\int_{\Omega}\left(\delta \Delta \hat{\mathbf{e}}^{\mathrm{T}} \mathbf{D} \Delta \hat{\mathbf{e}}\right) d \Omega & =\delta \Delta \hat{\mathbf{u}}\left[\int_{\Omega}\left(\mathbf{B}^{\mathrm{T}} \mathbf{D B}\right) d \Omega\right] \mathbf{T}^{\mathrm{T}} \Delta \hat{\mathbf{u}} \\
& =\delta \Delta \hat{\mathbf{U}}^{\mathrm{T}} \mathbf{T}\left[\int_{\Omega}\left(\mathbf{B}^{\mathrm{T}} \mathbf{D B}\right) d \Omega\right] \mathbf{T}^{\mathrm{T}} \Delta \hat{\mathbf{U}} \\
& =\delta \Delta \hat{\mathbf{U}}^{\mathrm{T}} \mathbf{K}_{L} \Delta \hat{\mathbf{U}}
\end{aligned}
$$

and

$$
\mathbf{K}_{L}=\mathbf{T} \mathbf{C}^{\mathrm{T}} \mathbf{A}^{-\mathrm{T}}\left[\int_{\Omega} \mathbf{P}^{\mathrm{T}} \mathbf{D P} d r d s\right] \mathbf{A}^{-1} \mathbf{C} \mathbf{T}^{\mathrm{T}} .
$$

The formulation of $\mathbf{K}_{L}$ may also be derived using the $\mathrm{Hu}$-Washizu variational principle. The equivalence of the quasi-conforming technique and the $\mathrm{Hu}$-Washizu derivation was shown by Chen [27]. Using the $\mathrm{Hu}$-Washizu variational principle, the 'generalized hybrid model' is derived, and (25) is obtained using the assumption $\mathbf{N}=\mathbf{P}_{\sigma}^{\mathrm{T}}=\mathbf{P}_{e}^{\mathrm{T}}=\mathbf{P}^{\mathrm{T}}$.

\subsection{Geometric Stiffness Matrix}

The geometric stiffness matrix is taken from the second term of the LHS of (22).

$$
\int_{\Omega}(\mathbf{S} \delta \Delta \hat{\mathbf{\Xi}}) d \Omega
$$

$\Delta \hat{\boldsymbol{\Xi}}$ is the incremental nonlinear part of the of the element strains. This results from the nonlinear part of the Green strain tensor and in cases when defined, the nonlinear parts of the displacement functions.

Equation (26) can be written in matrix form so that the quasi-conforming technique can be applied to derive the geometric stiffness. The nonlinear strain vector is broken down to its factors and defined as $\boldsymbol{\eta}$ and the stress vector is rearranged to a matrix form $\mathbf{F}_{g}$, the matrix integral is

$$
\int_{\Omega}(\mathbf{S} \delta \Delta \hat{\mathbf{\Xi}}) d \Omega=\int_{\Omega} \delta \Delta \eta \mathbf{F}_{g} \Delta \eta d \Omega
$$

Similar to the derivation for the linear strains, the strain vector is

$$
\begin{aligned}
\eta & =\mathbf{B} \mathbf{T}^{\mathrm{T}} \Delta \hat{\mathbf{U}} \\
& =\mathbf{P} \mathbf{A}^{-1} \mathbf{C} \mathbf{T}^{\mathrm{T}} \Delta \hat{\mathbf{U}} .
\end{aligned}
$$

Since element rank requirements have already been satisfied in the linear strain terms, in assigning values for $\mathbf{P}$, (13) does not have to be satisfied. In the formulation of the geometric stiffness, it is sufficient to use a constant nonlinear strain field distribution, i.e. $\mathbf{P}$ is a unit matrix. The solutions obtained using this assumption are shown to be stable and sufficient in linear and nonlinear buckling analysis and nonlinear material analysis with geometric nonlinearity.

$\mathbf{A}$ is then a diagonal matrix of the area of the reference surface and its inverse is trivial. In determining matrix $\mathbf{C}$, the same string functions used in the linear strain approximation is applied. This results in most of its terms being the same as its linear counterpart, which makes it convenient in derivation and implementation.

The resulting geometric stiffness is

$\mathbf{K}_{g}=\mathbf{T}\left\{\mathbf{C}^{\mathrm{T}} \mathbf{A}^{-\mathrm{T}}\left[\int_{\Omega} \mathbf{P}^{\mathrm{T}} \mathbf{F}_{g} \mathbf{P} d \Omega\right] \mathbf{A}^{-1} \mathbf{C}\right\} \mathbf{T}^{\mathrm{T}}$

\subsection{Internal Force Vector}

In order to properly analyze the motion of a structure, the internal forces must be correctly computed. The internal force vector is defined as the second term on the RHS of (22).

$\int_{\Omega}\left(\delta \Delta \hat{\mathbf{e}}^{\mathrm{T}} \mathbf{S}\right) d \Omega$

The quasi-conforming form of the resultant stresses are as defined in (18) and the linear strain terms are in (10). Substituting these into (30) gives the expression for the internal force vector.

$\mathbf{F}=\mathbf{T}\left\{\mathbf{C}^{\mathrm{T}} \mathbf{A}^{-\mathrm{T}}\left[\int_{\Omega} \mathbf{P}^{\mathrm{T}} \mathbf{D} \mathbf{P} d \Omega\right] \mathbf{A}^{-1} \mathbf{C}\right\} \mathbf{T}^{\mathrm{T}} \Delta \hat{\mathbf{U}}$.

The left and right A's and C's are the same. The evolution of plasticity or damage will be included in matrix $\mathbf{D}$.

\subsection{Mass Matrices}

There two formulations that are used to form mass matrices, the lumped mass and consistent mass matrix formulation. For the finite element formulations, the construction of the lumped mass matrix is straightforward. The total mass of the element is equally divided and lumped to the four nodes. Rotary inertia may be neglected, which would make the diagonal elements associated with rotational degrees of freedom zero. The lumped mass, $m$, for each translational degree of freedom is computed as

$m=\frac{1}{n} \int_{V} \rho d V=\frac{1}{n} M^{e}$,

$\rho$ is mass density and $M^{e}$ is the total mass of the elements.

When nodal forces are lumped, then it would be efficient to use the lumped mass matrix. The drawback of lumping is that if a coarse mesh is employed, the resulting solution may be very inaccurate. 


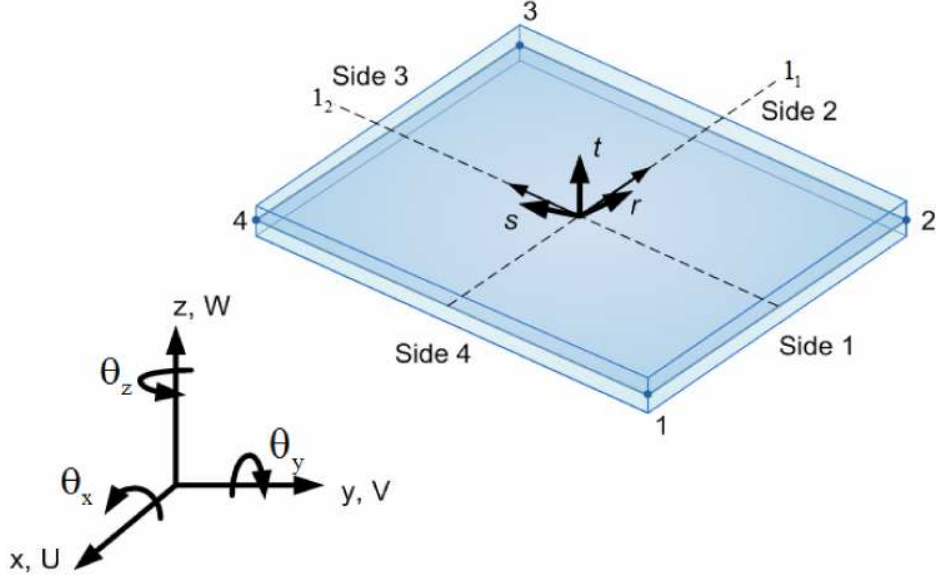

a)

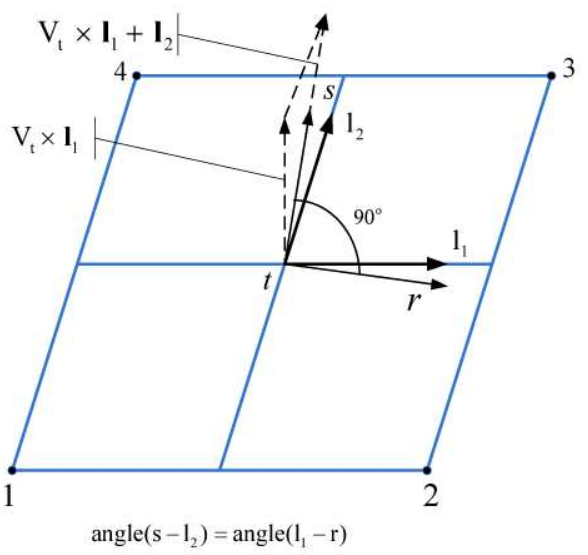

b)

Fig. 4 (a) Mid-surface geometry and local coordinates of 4-node shell element. (b) Plan of element with derivation of local coordinates

The consistent mass matrix formula is

$\mathbf{M}=\rho \int_{V} \mathbf{H}^{\mathrm{T}} \mathbf{H} d V$.

When consistent nodal loads are used, it is recommended that a consistent mass matrix be used. In the derivation of the consistent mass matrix, the interpolation functions in the formula come from the interpolation of the displacements and accelerations. This interpolation covers the entire element domain. In the quasi-conforming technique, the explicit definitions of displacements are only given along the boundaries. Therefore, it should be noted that the interpolation functions used in (33) should be compatible with the 'string functions'. When this is not the case, very good results are still possible when the loads and the mass matrices are consistent of finer meshes are used.

\section{Application of Quasi-Conforming Technique to Shell Element}

\subsection{Geometry of Shell Element}

A local orthogonal coordinate system $(r, s, t)$ is used to describe the geometry of the four-node shell element. Its origin is at the geometric center of the element. It is defined by first determining the unit vectors passing through the opposite midpoints of the shell mid-surface sides, $\mathbf{l}_{1}$ and $\mathbf{l}_{2}$, as shown in Fig. 4. The local coordinate base vectors $\mathbf{V}_{r}, \mathbf{V}_{s}$, $\mathbf{V}_{t}$ are then found by

$\mathbf{V}_{t}=\mathbf{l}_{1} \times \mathbf{l}_{2}, \quad \mathbf{V}_{s}=\frac{\left[\left(\mathbf{V}_{t} \times \mathbf{l}_{1}\right)+\mathbf{l}_{2}\right]}{\left\|\left(\mathbf{V}_{t} \times \mathbf{l}_{1}\right)+\mathbf{l}_{2}\right\|}$,

$\mathbf{V}_{r}=\mathbf{V}_{s} \times \mathbf{V}_{t}$ and the local coordinates of node $i, \mathbf{r}_{i}=\left(r_{i} s_{i} t_{i}\right)^{\mathrm{T}}$ are

$\mathbf{r}_{i}=\left[\begin{array}{lll}\mathbf{V}_{r} & \mathbf{V}_{s} & \mathbf{V}_{t}\end{array}\right]^{\mathrm{T}}\left(\mathbf{x}_{i}-\mathbf{x}_{c}\right)=\mathbf{T}^{\mathrm{T}}\left(\mathbf{x}_{i}-\mathbf{x}_{c}\right)$.

$\mathbf{x}_{i}$ and $\mathbf{x}_{c}$ are the vectors of the global coordinates of node $i$ and the element mid-surface center, respectively. The position vector of a point $\mathbf{r}(r, s, t)$ in the element is given by

$\mathbf{x}_{p}=\mathbf{x}_{c}+\mathbf{T r}$

Having defined the local coordinate base vectors, the transformation of the incremental displacement parameters $\Delta \overline{\mathbf{u}}_{i}, \Delta \varphi_{i}$ at node $i$ from the element local coordinates, to the global coordinates, is done by

$$
\begin{aligned}
\left\{\begin{array}{c}
\Delta \overline{\mathbf{u}} \\
\Delta \varphi
\end{array}\right\}_{i} & =\left\{\begin{array}{c}
\Delta \bar{u}_{i} \\
\Delta \bar{v}_{i} \\
\Delta \bar{w}_{i} \\
\Delta \varphi_{r i} \\
\Delta \varphi_{s i} \\
\Delta \varphi_{t i}
\end{array}\right\}=\left[\begin{array}{cc}
\mathbf{T}^{\mathrm{T}} & 0 \\
0 & \mathbf{T}^{\mathrm{T}}
\end{array}\right]\left\{\begin{array}{c}
\Delta \bar{U}_{i} \\
\Delta \bar{V}_{i} \\
\Delta \bar{W}_{i} \\
\Delta \theta_{x i} \\
\Delta \theta_{y i} \\
\Delta \theta_{z i}
\end{array}\right\} \\
& =\mathbf{T}_{g i}^{\mathrm{T}}\left\{\begin{array}{c}
\Delta \overline{\mathbf{U}} \\
\Delta \boldsymbol{\theta}
\end{array}\right\}_{i} .
\end{aligned}
$$

The bar denotes, measured from the mid-surface. Transformation matrix $\mathbf{T}$ is defined as

$\mathbf{T}=\left[\begin{array}{lll}\mathbf{V}_{r} & \mathbf{V}_{s} & \mathbf{V}_{t}\end{array}\right]$

It is important to note that $\mathbf{V}_{t}$ is normal to the mid-surface of the element and it is independent of the top and the bottom nodal coordinates. The approximation introduced by ignoring variation of Jacobian through the thickness would not violate rigid body rotation requirement if $\mathbf{V}_{t}$ is used in the definition of the geometry of the elements instead of $\mathbf{V}_{t i}$, which depends on the top and bottom nodal coordinates. 
As shown in Fig. 4a, the shell element geometry considers a flat surface. For concrete shell structures with cylindrical or doubly curved geometries, the present element would approximate the surface using a mesh of flat surfaces. For the case of a warped or twisted geometry, the warping correction suggested in Taylor [154] can be applied. The transformation matrix $\mathbf{T}_{g i}$, for this case then becomes

$\mathbf{T}_{g i}=\left[\begin{array}{cccccc}\mathbf{V}_{r} & \mathbf{V}_{s} & \mathbf{V}_{t} & \mathbf{0} & \mathbf{0} & \mathbf{0} \\ -\Delta \cdot \mathbf{V}_{s} & \Delta \cdot \mathbf{V}_{r} & \mathbf{0} & \mathbf{V}_{r} & \mathbf{V}_{s} & \mathbf{V}_{t}\end{array}\right]$

where $\Delta$ is the distance from node to the shell mid-surface.

\subsection{Co-rotational Displacements}

The transformation from the initial local co-ordinate system ${ }^{o} r,{ }^{o} s$ and ${ }^{o} t$ to the current local co-ordinate system state ${ }^{t} r,{ }^{t} s$ and ${ }^{t} t$ is achieved by the orthogonal rotation matrix, $\mathbf{R}(\widehat{\varphi})$, corresponding to the rigid body rotation in local coordinates. $\mathbf{R}(\widehat{\varphi})$ can be considered as a transformation matrix of any point with position vector in unconvected state to a new position vector in the convected state. (In the following, the notation $\wedge$ will be used for the co-rotational value attributed to pure deformation. And the superscript $t$ denotes current configuration will be ignored):

$\mathbf{T}=\mathbf{R}(\widehat{\varphi}) \bullet{ }^{0} \mathbf{T}$.

${ }^{0} \mathbf{T}$ and $\mathbf{T}$ are the standard transformation matrices that are defined in (38), in the initial and current configuration respectively.

The co-rotational (or convective) displacement for any node $i$ is defined as a residual displacement that removes the rigid body motion that occurs at the center of the element, and represent the motion of any node relative to the motion of the point defined by $r=s=0$. The co-rotational nodal displacement $(\hat{\mathbf{u}})$ associated with pure deformation can be expressed as follows:

$\hat{\mathbf{u}}=\left(\mathbf{x}_{i}-\mathbf{x}_{c}\right)-\mathbf{R}(\widehat{\varphi})\left({ }^{0} \mathbf{x}_{i}-{ }^{0} \mathbf{x}_{c}\right)$.

The above equation (41) represents the co-rotational displacements by the position vector of any node $i$ relative to the element center $c$ in the current and initial configuration. In accordance with polar decomposition theory, the total rotation can be decomposed into rigid body rotation $\widehat{\varphi}$ followed by a small transverse shear deformation $\hat{\varphi}$, that is to say

$\mathbf{R}(\varphi)=\mathbf{R} r(\hat{\varphi}) \mathbf{R}(\widehat{\varphi})$.

The local components of $\hat{\varphi}$ can be identified with the average shear deformation of the cross section, that is $\hat{\varphi}=$ $\left\{0 \hat{\varphi}_{s} \hat{\varphi}_{t}\right\}=\left\{0 \hat{\bar{\varepsilon}}_{r t}-\hat{\bar{\varepsilon}}_{r s}\right\}$.

When the residual rotations are small enough to be vector quantity $\left(\hat{\varphi}_{i}<0.02\right)$, the convective nodal rotation $\hat{\varphi}_{i}$ can be obtained by removing the rigid body rotation $(\widehat{\varphi})$ from the total nodal rotation and approximated as follows:

$\hat{\varphi}_{i}=\varphi_{i}-\widehat{\varphi}$.

The local kinematic relations based on the co-rotational displacement can be expressed as follows:

$\Delta \hat{u}=\Delta \hat{\bar{u}}+t\left(\Delta \hat{\varphi}_{s}+1 / 2 \Delta \hat{\varphi}_{r} \Delta \hat{\varphi}_{t}\right)$,

$\Delta \hat{v}=\Delta \hat{\bar{v}}-t\left(\Delta \hat{\varphi}_{r}-1 / 2 \Delta \hat{\varphi}_{s} \Delta \hat{\varphi}_{t}\right)$,

$\Delta \hat{w}=\Delta \hat{\bar{w}}(r, s)$.

Further detailed discussion about the co-rotational formulation adopted is found in references $[11,80]$.

\subsection{Strain-Displacement Relationships}

In degenerated formulations based on continuum mechanics theory, the geometrical non-linearity arises due to both the quadratic terms of the Green strain tensor and the kinematic relation themselves. Following classical nonlinear thin shell theory and substituting the co-rotational kinematic relations given by (44), the incremental membrane $\Delta \hat{\overline{\boldsymbol{\varepsilon}}}_{m}=$ $\left\{\Delta \hat{\bar{\varepsilon}}_{r} \Delta \hat{\bar{\varepsilon}}_{s} \Delta \hat{\bar{\varepsilon}}_{r s}\right\}^{\mathrm{T}}$, bending $\Delta \hat{\boldsymbol{\varepsilon}}_{b}=\left\{\Delta \hat{\chi}_{r} \Delta \hat{\chi}_{s} \Delta \hat{\chi}_{r s}\right\}^{\mathrm{T}}$ and transverse shear $\Delta \hat{\boldsymbol{\varepsilon}}_{q}=\left\{\Delta \hat{\varepsilon}_{r t} \Delta \hat{\varepsilon}_{s t}\right\}^{\mathrm{T}}$ strains on the midsurface are obtained as in Table 1 .

When the rotation $\varphi_{t}$ due to the quadratic terms in the nonlinear kinematic relations is dropped, the geometric stiffness will be incomplete and certain forms of buckling behaviour will be precluded. The above expressions are consistent with Novozhilov's non-linear theory (1963) [98], which states that the curvature terms are quadratic functions of the rotational parameters. In addition, the non-linear terms in transverse shear strains are also accounted for.

The incremental membrane, bending and transverse shear strains represented in (45) to (47) can be separated into linear and non-linear parts, $\Delta \hat{\boldsymbol{\varepsilon}}_{m}=\Delta \hat{\mathbf{e}}_{m}+\Delta \hat{\mathbf{\Xi}}^{m}, \Delta \hat{\boldsymbol{\varepsilon}}_{b}=$ $\Delta \hat{\mathbf{e}}_{b}+\Delta \hat{\boldsymbol{\Xi}}^{b}, . \Delta \hat{\boldsymbol{\varepsilon}}_{q}=\Delta \hat{\mathbf{e}}_{q}+\Delta \hat{\boldsymbol{\Xi}}^{q}$

Since a degenerated concept was adopted in the formulation of the shell elements, the bar symbol over the displacements, $u, v$ and $w$, were placed to denote as being measured from the mid-surface of the element (45).

\subsection{Equation of Motion}

In formulating the shell elements, an updated Lagrangian formulation is adopted. The linearized equation of motion is given in (21). Assuming constant thickness during deformation, (21) for the shell elements may be expressed as

$$
\begin{aligned}
\int_{A}(\delta \Delta & \hat{\mathbf{e}}_{m}^{\mathrm{T}} \mathbf{D}_{m} \Delta \hat{\mathbf{e}}_{m}+\delta \Delta \hat{\mathbf{e}}_{m}^{\mathrm{T}} \mathbf{D}_{m b} \Delta \hat{\mathbf{e}}_{b}+\delta \Delta \hat{\mathbf{e}}_{b}^{\mathrm{T}} \mathbf{D}_{m b} \Delta \hat{\mathbf{e}}_{m} \\
+ & \left.\delta \Delta \hat{\mathbf{e}}_{b}^{\mathrm{T}} \mathbf{D}_{b} \Delta \hat{\mathbf{e}}_{b}+\delta \Delta \hat{\mathbf{e}}_{q}^{\mathrm{T}} \mathbf{D}_{q} \Delta \hat{\mathbf{e}}_{q}\right) d r d s
\end{aligned}
$$


Table 1 Shell strain terms

Linear term (e)

Non-linear term $(\Xi)$

Membrane strains

$$
\begin{array}{ll}
\Delta \hat{\bar{\varepsilon}}_{r}=\frac{\partial \Delta \hat{\bar{u}}}{\partial r} & +\frac{1}{2}\left[\left(\frac{\partial \Delta \hat{\bar{u}}}{\partial r}\right)^{2}+\left(\frac{\partial \Delta \hat{\bar{v}}}{\partial r}\right)^{2}+\left(\frac{\partial \Delta \hat{\bar{w}}}{\partial r}\right)^{2}\right] \\
\Delta \hat{\bar{\varepsilon}}_{s}=\frac{\partial \Delta \hat{\bar{v}}}{\partial s} & +\frac{1}{2}\left[\left(\frac{\partial \Delta \hat{\bar{u}}}{\partial s}\right)^{2}+\left(\frac{\partial \Delta \hat{\bar{v}}}{\partial s}\right)^{2}+\left(\frac{\partial \Delta \hat{\bar{w}}}{\partial s}\right)^{2}\right] \\
\Delta \hat{\bar{\varepsilon}}_{r s}=\frac{\partial \Delta \hat{\bar{u}}}{\partial s}+\frac{\partial \Delta \hat{\bar{v}}}{\partial r} & +\frac{\partial \Delta \hat{\bar{u}}}{\partial r} \frac{\partial \Delta \hat{\bar{u}}}{\partial s}+\frac{\partial \Delta \hat{\bar{v}}}{\partial r} \frac{\partial \Delta \hat{\bar{v}}}{\partial s}+\frac{\partial \Delta \hat{\bar{w}}}{\partial r} \frac{\partial \Delta \hat{\bar{w}}}{\partial s}
\end{array}
$$

Bending strains

$$
\begin{aligned}
& \Delta \hat{\kappa}_{r}=\frac{\partial \Delta \hat{\varphi}_{s}}{\partial r} \\
& \Delta \hat{\kappa}_{s}=-\frac{\partial \Delta \hat{\varphi}_{r}}{\partial s} \\
& \Delta \hat{\kappa}_{r s}=\frac{\partial \Delta \hat{\varphi}_{s}}{\partial s}-\frac{\partial \Delta \hat{\varphi}_{r}}{\partial r}
\end{aligned}
$$$$
+\frac{\partial \Delta \hat{\bar{u}}}{\partial r} \frac{\partial \Delta \hat{\varphi}_{s}}{\partial r}-\frac{\partial \Delta \hat{\bar{v}}}{\partial r} \frac{\partial \Delta \hat{\varphi}_{r}}{\partial r}+\frac{1}{2}\left[\Delta \hat{\varphi}_{r} \frac{\partial \Delta \hat{\varphi}_{t}}{\partial r}+\Delta \hat{\varphi}_{t} \frac{\partial \Delta \hat{\varphi}_{r}}{\partial r}\right]
$$$$
+\frac{\partial \Delta \hat{\bar{u}}}{\partial s} \frac{\partial \Delta \hat{\varphi}_{s}}{\partial s}-\frac{\partial \Delta \hat{\bar{v}}}{\partial s} \frac{\partial \Delta \hat{\varphi}_{r}}{\partial s}+\frac{1}{2}\left[\Delta \hat{\varphi}_{s} \frac{\partial \Delta \hat{\varphi}_{t}}{\partial s}+\Delta \hat{\varphi}_{t} \frac{\partial \Delta \hat{\varphi}_{s}}{\partial s}\right]
$$

$$
\begin{aligned}
& +\frac{\partial \Delta \hat{\bar{u}}}{\partial r} \frac{\partial \Delta \hat{\varphi}_{s}}{\partial s}+\frac{\partial \Delta \hat{\bar{u}}}{\partial s} \frac{\partial \Delta \hat{\varphi}_{s}}{\partial r}-\frac{\partial \Delta \hat{\bar{v}}}{\partial s} \frac{\partial \Delta \hat{\varphi}_{r}}{\partial r}-\frac{\partial \Delta \hat{\bar{v}}}{\partial r} \frac{\partial \Delta \hat{\varphi}_{r}}{\partial s} \\
& +\frac{1}{2}\left[\Delta \hat{\varphi}_{r} \frac{\partial \Delta \hat{\varphi}_{t}}{\partial s}+\Delta \hat{\varphi}_{s} \frac{\partial \Delta \hat{\varphi}_{t}}{\partial r}+\Delta \hat{\varphi}_{t} \frac{\partial \Delta \hat{\varphi}_{r}}{\partial s}+\Delta \varphi_{t} \frac{\partial \Delta \hat{\varphi}_{s}}{\partial r}\right]
\end{aligned}
$$

Transverse shear strains

$$
\begin{array}{ll}
\Delta \hat{\varepsilon}_{r t}=\frac{\partial \Delta \hat{\bar{w}}}{\partial r}+\Delta \hat{\varphi}_{s} & +\frac{\partial \Delta \hat{\bar{u}}}{\partial r} \Delta \hat{\varphi}_{s}-\frac{\partial \Delta \hat{\bar{v}}}{\partial r} \Delta \hat{\varphi}_{r}+\frac{1}{2} \Delta \hat{\varphi}_{r} \Delta \hat{\varphi}_{t} \\
\Delta \hat{\varepsilon}_{s t}=\frac{\partial \Delta \hat{\bar{w}}}{\partial s}-\Delta \hat{\varphi}_{r} & +\frac{\partial \Delta \hat{\bar{u}}}{\partial s} \Delta \hat{\varphi}_{s}-\frac{\partial \Delta \hat{\bar{v}}}{\partial s} \Delta \hat{\varphi}_{r}+\frac{1}{2} \Delta \hat{\varphi}_{s} \Delta \hat{\varphi}_{t}
\end{array}
$$

$$
\begin{gathered}
+\int_{A}\left(\mathbf{N} \delta \Delta \hat{\mathbf{\Xi}}_{m}+\mathbf{M} \delta \Delta \hat{\mathbf{\Xi}}_{b}+\mathbf{Q} \delta \Delta \hat{\mathbf{\Xi}}_{q}\right) d r d s \\
={ }^{t+\Delta t} \Re-\int_{A}\left(\delta \Delta \hat{\mathbf{e}}_{b}^{\mathrm{T}} \mathbf{M}+\delta \Delta \hat{\mathbf{e}}_{m}^{\mathrm{T}} \mathbf{N}+\delta \Delta \hat{\mathbf{e}}_{q}^{\mathrm{T}} \mathbf{Q}\right) d r d s .
\end{gathered}
$$

$\mathbf{D}_{\mathrm{b}}, \mathbf{D}_{\mathrm{m}}, \mathbf{D}_{\mathrm{q}}$ and $\mathbf{D}_{\mathrm{mb}}$ are flexural, extensile, transverse shear and coupled membrane-bending rigidity matrices. $\mathbf{M}, \mathbf{N}$ and $\mathbf{Q}$ are the stress couple, in-plane stress and transverse shear resultant vectors.

\subsection{Stiffness Matrix Due to Linear Strains}

The first term on the LHS, where the stiffness matrix due to linear strains is derived, is

$$
\begin{aligned}
& \delta \Delta \hat{\mathbf{e}}_{m}^{\mathrm{T}} \mathbf{D}_{m} \Delta \hat{\mathbf{e}}_{m}+\delta \Delta \hat{\mathbf{e}}_{m}^{\mathrm{T}} \mathbf{D}_{m b} \Delta \hat{\mathbf{e}}_{b}+\delta \Delta \hat{\mathbf{e}}_{b}^{\mathrm{T}} \mathbf{D}_{m b} \Delta \hat{\mathbf{e}}_{m} \\
& \quad+\delta \Delta \hat{\mathbf{e}}_{b}^{\mathrm{T}} \mathbf{D}_{b} \Delta \hat{\mathbf{e}}_{b}+\delta \Delta \hat{\mathbf{e}}_{q}^{\mathrm{T}} \mathbf{D}_{q} \Delta \hat{\mathbf{e}}_{q} .
\end{aligned}
$$

The three linear strains $\Delta \hat{\mathbf{e}}_{m}, \Delta \hat{\mathbf{e}}_{b}$ and $\Delta \hat{\mathbf{e}}_{q}$ are defined independently with the quasi-conforming technique. The bending and transverse shear strain for the two shell elements use the same string functions. The membrane strains of the three-node shell are defined with the Allman (1984) [3] string function. The Allman string function directly incorporates the drilling dof into the striffness formulation. For the four-node shell element the membrane strains use simple purely translation boundary displacement function. The drilling dof is added using a strain energy function.

As discussed in Sects. 2.1 and 2.3, the strains may be defined in terms of displacements.

$\hat{\boldsymbol{\varepsilon}}=\mathbf{B}\left\{\begin{array}{l}\hat{\hat{\mathbf{u}}} \\ \hat{\varphi}\end{array}\right\}=\mathbf{P A}^{-1} \mathbf{C}\left\{\begin{array}{l}\hat{\hat{\mathbf{u}}} \\ \hat{\varphi}\end{array}\right\}$,

$\mathbf{A}=\int_{\Omega}\left[\mathbf{P}^{\mathrm{T}} \mathbf{P}\right] d \Omega \quad$ and $\quad \mathbf{C}\left[\begin{array}{c}\overline{\mathbf{u}} \\ \varphi\end{array}\right]=\int_{\Omega}\left[\mathbf{P}^{\mathrm{T}} \boldsymbol{\varepsilon}\right] d \Omega$.

In terms of the global displacement vector, (50) is written as

$\hat{\boldsymbol{\varepsilon}}=\mathbf{B} \mathbf{T}_{g}^{\mathrm{T}}\left\{\begin{array}{l}\hat{\overline{\mathbf{U}}} \\ \hat{\boldsymbol{\theta}}\end{array}\right\}=\mathbf{P A}^{-1} \mathbf{C} \mathbf{T}_{g}^{\mathrm{T}}\left\{\begin{array}{l}\hat{\overline{\mathbf{U}}} \\ \hat{\boldsymbol{\theta}}\end{array}\right\}$. 
For the four-node shell, the bending strains require eleven strain parameters.

$$
\begin{aligned}
\hat{\mathbf{e}}_{b} & =\left\{\begin{array}{c}
\frac{\partial \hat{\varphi}_{s}}{\partial r} \\
-\frac{\partial \hat{\varphi}_{r}}{\partial s} \\
\frac{\partial \hat{\varphi}_{s}}{\partial r}-\frac{\partial \hat{\varphi}_{r}}{\partial s}
\end{array}\right\}=\mathbf{P}_{b} \boldsymbol{\alpha}_{b} \\
& =\left[\begin{array}{ccc}
1 r s r s & 0 & 0 \\
0 & 1 r s r s & 0 \\
0 & 0 & 1 r s
\end{array}\right]\left\{\begin{array}{c}
\alpha_{1} \\
: \\
\alpha_{11}
\end{array}\right\} .
\end{aligned}
$$

By using the Green's theorem and the direction cosines shown in Fig. 2, the first four integrals of $\mathbf{C}_{b}\left\{\begin{array}{c}\hat{\overline{\mathbf{u}}} \\ \hat{\varphi}\end{array}\right\}=$ $\int_{A} \mathbf{P}_{b}^{\mathrm{T}} \hat{\mathbf{e}}_{b} d r d s$ can be solved as

$$
\begin{aligned}
& \mathbf{C}_{b}(1, j)\left\{\begin{array}{l}
\hat{\overline{\mathbf{u}}} \\
\hat{\varphi}
\end{array}\right\} \\
& =\int_{A} \frac{\partial \hat{\varphi}_{s}}{\partial r} d r d s=\oint \hat{\varphi}_{s} n_{r} d L \quad(j=1,2, \ldots, \\
& =\oint\left[\hat{\varphi}_{n} n_{s}+\hat{\varphi}_{t} n_{r}\right] n_{r} d L \\
& =\sum_{k=1}^{4}\left[n_{r k} n_{s k} \int_{L k} \hat{\varphi}_{n} d L+n_{r k}^{2} \int_{L k} \hat{\varphi}_{t} d L\right], \\
& \mathbf{C}_{b}(2, j)\left\{\begin{array}{l}
\hat{\overline{\mathbf{u}}} \\
\hat{\varphi}
\end{array}\right\} \\
& =\int_{A} \frac{\partial \hat{\varphi}_{s}}{\partial r} r d r d s=\oint \hat{\varphi}_{s} r n_{r} d L-\int_{A} \hat{\varphi}_{s} d r d s \\
& =\oint\left[\hat{\varphi}_{n} n_{r} n_{s}+\hat{\varphi}_{t} n_{r}^{2}\right] r d L-\int_{A} \hat{\varphi}_{s} d r d s, \\
& \mathbf{C}_{b}(3, j)\left\{\begin{array}{l}
\hat{\overline{\mathbf{u}}} \\
\hat{\varphi}
\end{array}\right\} \\
& =\int_{A} \frac{\partial \hat{\varphi}_{s}}{\partial r} s d r d s=\oint\left[\hat{\varphi}_{n} n_{r} n_{s}+\hat{\varphi}_{t} n_{r}^{2}\right] s d L, \\
& \mathbf{C}_{b}(4, j)\left\{\begin{array}{l}
\hat{\overline{\mathbf{u}}} \\
\hat{\varphi}
\end{array}\right\} \\
& =\int_{A} \frac{\partial \hat{\varphi}_{s}}{\partial r} r s d r d s \quad(j=1,2, \ldots, 24) \\
& =\oint\left[\hat{\varphi}_{n} n_{r} n_{s}+\hat{\varphi}_{t} n_{r}^{2}\right] r s d L-\int_{A} \hat{\varphi}_{s} s d r d s .
\end{aligned}
$$

$k$ indicates the side number where the line integral is being taken.

Noting that, $n_{r}$ and $n_{s}$ in (54) are the side normal and tangent as defined in (55) and Fig. 2, which are different from the base vector components in (38).

$$
\left[\begin{array}{l}
\hat{\varphi}_{r} \\
\hat{\varphi}_{s}
\end{array}\right]=\left[\begin{array}{cc}
n_{r} & -n_{s} \\
n_{s} & n_{r}
\end{array}\right]\left[\begin{array}{c}
\hat{\varphi}_{n} \\
\hat{\varphi}_{t}
\end{array}\right] .
$$

The line integrals are made along each side, i.e. from node to node. They are directly solved using the string functions introduced by Shi and Voyiadjis [132-134]. The string functions defined based on nodal values of displacement $w$ and normal rotation $\hat{\varphi}_{n}$ are

$$
\begin{aligned}
\hat{\varphi}_{n}(\xi)= & -\frac{3}{2 l} \lambda\left(1-\xi^{2}\right) \hat{\bar{w}}_{i}+\frac{1}{4}\left[2-2 \xi-3 \lambda\left(1-\xi^{2}\right)\right] \hat{\varphi}_{n i} \\
& +\frac{3}{2 l} \lambda\left(1-\xi^{2}\right) \hat{\bar{w}}_{j}+\frac{1}{4}\left[2+2 \xi-3 \lambda\left(1-\xi^{2}\right)\right] \hat{\varphi}_{n j},
\end{aligned}
$$

$$
\begin{aligned}
\hat{\bar{w}}(\xi)= & \frac{1}{2}\left[1-\xi+\frac{\lambda}{2}\left(\xi^{3}-\xi\right)\right] \hat{\bar{w}}_{i} \\
& +\frac{1}{4}\left[1-\xi^{2}+\lambda\left(\xi^{3}-\xi\right)\right] \frac{l}{2} \hat{\varphi}_{n i} \\
& +\frac{1}{2}\left[1+\xi-\frac{\lambda}{2}\left(\xi^{3}-\xi\right)\right] \hat{\bar{w}}_{j} \\
& +\frac{1}{4}\left[-1+\xi^{2}+\lambda\left(\xi^{3}-\xi\right)\right] \frac{l}{2} \hat{\varphi}_{n j}
\end{aligned}
$$

where $l$ is the distance from node $i$ to node $j$ and

$\xi=\frac{1}{l}(2 S-l) \quad$ for $0 \leq S \leq l,-1 \leq \xi \leq 1$,

$\lambda=\frac{1}{1+12 \frac{D_{b 11}}{D_{q 11} L^{2}}}$,

$\hat{\varphi}_{t}(\xi)=\frac{1}{2}(1-\xi) \hat{\varphi}_{t i}+\frac{1}{2}(1+\xi) \hat{\varphi}_{t j}$.

$D_{b 11}, D_{q 11}$ and $L$ are, respectively, the first component of the flexural rigidity, the first component of the transverse shear rigidity and side length. $S$ is the side local coordinate, 0 at node $i$ and $l$ at node $j$.

Area integrals still present in (54) can be approximated as:

$$
\begin{aligned}
& \int_{A} \hat{\varphi}_{s}\left(\hat{\bar{w}}_{i}, \hat{\varphi}_{r i}, \hat{\varphi}_{s i}\right) d r d s \\
& \cong \int_{A}\left[-\lambda_{r} \frac{\partial \hat{\bar{w}}}{\partial r}+\left(1-\lambda_{r}\right) \hat{\varphi}_{s}\left(\hat{\varphi}_{s i}\right)\right] d r d s \\
& \quad=-\lambda_{r} \oint \hat{\bar{w}} n_{r} d s+\left(1-\lambda_{r}\right) \int_{A} \hat{\varphi}_{s}\left(\hat{\varphi}_{s i}\right) d r d s,
\end{aligned}
$$

$\int_{A} \hat{\varphi}_{S}\left(\hat{\bar{w}}_{i}, \hat{\varphi}_{r i}, \hat{\varphi}_{s i}\right) d r d s$

$$
=\lambda_{s} \oint \hat{\bar{w}} n_{s} d s+\left(1-\lambda_{s}\right) \int_{A} \hat{\varphi}_{r}\left(\hat{\varphi}_{r i}\right) d r d s
$$

in which $w$ is integrated using the definition in (57). This approximation is applicable for thick shells and also satisfies the Kirchhoff assumption for the case of thin shells. The remaining area integral is integrated by isoparametric mapping. 
The transverse shear strains in (47) are approximated as:

$\hat{\mathbf{e}}_{q}=\left[\begin{array}{c}\frac{\partial \hat{\bar{w}}}{\partial r}+\hat{\varphi}_{s} \\ \frac{\partial \hat{\bar{w}}}{\partial s}-\hat{\varphi}_{r}\end{array}\right]=\mathbf{P}_{q} \boldsymbol{\alpha}_{q}=\left[\begin{array}{ll}1 & \\ & 1\end{array}\right]\left[\begin{array}{l}\alpha_{10} \\ \alpha_{11}\end{array}\right]$.

The integrals involved in determining $\mathbf{C}_{q}$ are

$$
\begin{aligned}
C_{s 1 j}\left\{\begin{array}{c}
\hat{\overline{\mathbf{u}}} \\
\hat{\varphi}
\end{array}\right\}= & \int_{A}\left[\frac{\partial \hat{\bar{w}}}{\partial r}+\hat{\varphi}_{s}\right] d r d s \\
= & \oint \hat{\bar{w}} n_{r} d L-\lambda_{r} \oint \hat{\bar{w}} n_{r} d L \\
& +\left(1-\lambda_{r}\right) \int_{A} \hat{\varphi}_{s} d r d s, \\
C_{s 2 j}\left\{\begin{array}{l}
\hat{\overline{\mathbf{u}}} \\
\hat{\varphi}
\end{array}\right\}= & \int_{\Omega}\left[\frac{\partial \hat{\bar{w}}}{\partial s}+\hat{\varphi}_{r}\right] d r d s \\
= & \oint \hat{\bar{w}} n_{s} d L-\lambda_{s} \oint \hat{\bar{w}} n_{s} d L \\
& -\left(1-\lambda_{s}\right) \int_{\Omega} \hat{\varphi}_{r} d r d s .
\end{aligned}
$$

The A matrices for the bending, transverse shear and membrane strains are composed only of polynomials. For example, using the definition of $\mathbf{P}_{b}$ in (53), matrix $\mathbf{A}_{b}$ has the form

$$
\begin{gathered}
\mathbf{A}_{b}=\left[\begin{array}{ccc}
\mathbf{A}_{11} & & 0 \\
& \mathbf{A}_{11} & \\
0 & & \mathbf{A}_{11}
\end{array}\right], \quad \text { where } \\
\mathbf{A}_{11}=\int_{A}\left[\begin{array}{ccc}
1 & r & s \\
& r^{2} & r s \\
\text { Sym. } & & s^{2}
\end{array}\right] d r d s .
\end{gathered}
$$

The area integrals can be evaluated by line integrals by (15).

For the membrane component of the linear stiffness matrix, the Allman (1984) [3] membrane formulation is adopted. The membrane strains are approximated with

$$
\hat{\mathbf{e}}_{m}=\left\{\begin{array}{c}
\frac{\partial \hat{\bar{u}}}{\partial r} \\
\frac{\partial \hat{\bar{v}}}{\partial s} \\
\frac{\partial \hat{\bar{u}}}{\partial s}+\frac{\partial \hat{\bar{v}}}{\partial r}
\end{array}\right\}=\mathbf{P}_{m} \boldsymbol{\alpha}_{m}=\left[\begin{array}{ccccc}
1 & s & 0 & 0 & 0 \\
0 & 0 & 1 & r & 0 \\
0 & 0 & 0 & 0 & 1
\end{array}\right]\left\{\begin{array}{c}
\alpha_{14} \\
\vdots \\
\alpha_{18}
\end{array}\right\}
$$

and the string functions used for the displacements are

$$
\begin{aligned}
& \hat{\bar{u}}=\frac{1}{2}(1-\xi) \hat{\bar{u}}_{i}+\frac{1}{2}(1+\xi) \hat{\bar{u}}_{j}, \\
& \hat{\bar{v}}=\frac{1}{2}(1-\xi) \hat{\bar{v}}_{i}+\frac{1}{2}(1+\xi) \hat{\bar{v}}_{j} .
\end{aligned}
$$

To model stiffened plates and shells, the need for six degrees of freedom arises because the process of stiffness accumulation at any node lying on the junction must be carried out in a single reference frame. However, it is well known that this creates problems associated with rotation about the normal to the shell mid-surface. Initially, this torsional degree of freedom is specified in global co-ordinates but this leads to singularity problems when adjacent elements are exactly co-planar. The difficulty is overcome by providing a fictitious torsional spring along the local normal direction at each node of the element. However, this technique has the drawback that it interferes with the ability of the element to undergo strain free rigid body motions. Kanok-Nukulchai [77] used an additional constraint to link the torsional rotation $\left(\varphi_{t}\right)$ to the average in-plane rotation of the mid-surface. Adopting the continuum mechanics definition, the constraint equation can be written as

$E_{d}=\hat{\varphi}_{t}-\frac{1}{2}\left(\frac{\partial \hat{\bar{v}}}{\partial r}-\frac{\partial \hat{\bar{u}}}{\partial s}\right)=0$

The rotation of the normal to the mid-surface is assumed to have the governing strain energy

$\pi_{t}=k_{t} A_{33} h \int_{A}\left[\hat{\varphi}_{t}-\frac{1}{2}\left(\frac{\partial \hat{\bar{v}}}{\partial r}-\frac{\partial \hat{\bar{u}}}{\partial s}\right)\right]^{2} d r d s$.

In applying the QCT, the drilling strain is taken as $\hat{\varepsilon}_{d}=\varphi_{t}-$ $\frac{1}{2}\left(\frac{\partial \hat{\bar{v}}}{\partial r}-\frac{\partial \hat{\bar{u}}}{\partial s}\right)$ and a constant strain field is assumed, $\mathrm{P}_{d}=1$. This would result in

$$
\begin{aligned}
\mathbf{C}_{d 1 j}\left\{\begin{array}{c}
\hat{\overline{\mathbf{u}}} \\
\hat{\varphi}
\end{array}\right\}= & \int_{A} P_{d}^{\mathrm{T}} \hat{\varepsilon}_{d} d r d s \\
= & \int_{A} 1 \times\left[\hat{\varphi}_{t}-\frac{1}{2}\left(\frac{\partial \hat{\bar{v}}}{\partial r}-\frac{\partial \hat{\bar{u}}}{\partial s}\right)\right] d r d s \\
= & \int_{-1}^{1} \int_{-1}^{1} \hat{\varphi}_{t}(\xi, \eta)|J| d \xi d \eta \\
& -\frac{1}{2} \oint\left(\hat{\bar{v}} n_{r}-\hat{\bar{u}} n_{s}\right) d L .
\end{aligned}
$$

Taking the first expression in (48) and augmenting it with (69) obtains the stiffness matrix due to linear strains.

$$
\begin{aligned}
\mathbf{K}_{L}= & \mathbf{T}_{g} \int_{A}\left(\mathbf{B}_{m}^{\mathrm{T}} \mathbf{D}_{m} \mathbf{B}_{m}+\mathbf{B}_{m}^{\mathrm{T}} \mathbf{D}_{m b} \mathbf{B}_{b}\right. \\
& +\mathbf{B}_{b}^{\mathrm{T}} \mathbf{D}_{m b} \mathbf{B}_{m}+\mathbf{B}_{b}^{\mathrm{T}} \mathbf{D}_{b} \mathbf{B}_{b} \\
& \left.+\mathbf{B}_{q}^{\mathrm{T}} \mathbf{D}_{q} \mathbf{B}_{q}+\mathbf{B}_{d}^{\mathrm{T}} D_{m 33} h \mathbf{B}_{d}\right) d r d s \mathbf{T}_{g}^{\mathrm{T}} \\
\mathbf{K}_{L}= & \mathbf{T}_{g}\left\{\mathbf{C}_{m}^{\mathrm{T}} \mathbf{A}_{m}^{-\mathrm{T}}\left[\int_{A} \mathbf{P}_{m}^{\mathrm{T}} \mathbf{D}_{m} \mathbf{P}_{m} d r d s\right] \mathbf{A}_{m}^{-1} \mathbf{C}_{m}\right. \\
& +\mathbf{C}_{m}^{\mathrm{T}} \mathbf{A}_{m}^{-\mathrm{T}}\left[\int_{A} \mathbf{P}_{m}^{\mathrm{T}} \mathbf{D}_{m b} \mathbf{P}_{b} d r d s\right] \mathbf{A}_{b}^{-1} \mathbf{C}_{b} \\
& +\mathbf{C}_{b}^{\mathrm{T}} \mathbf{A}_{b}^{-\mathrm{T}}\left[\int_{A} \mathbf{P}_{b}^{\mathrm{T}} \mathbf{D}_{m b} \mathbf{P}_{m} d r d s\right] \mathbf{A}_{m}^{-1} \mathbf{C}_{m}
\end{aligned}
$$




$$
\begin{aligned}
& +\mathbf{C}_{b}^{\mathrm{T}} \mathbf{A}_{b}^{-\mathrm{T}}\left[\int_{A} \mathbf{P}_{b}^{\mathrm{T}} \mathbf{D}_{b} \mathbf{P}_{b} d r d s\right] \mathbf{A}_{b}^{-1} \mathbf{C}_{b} \\
& \left.+\mathbf{C}_{q}^{\mathrm{T}} \mathbf{A}_{q}^{-\mathrm{T}}\left[\int_{A} \mathbf{P}_{q}^{\mathrm{T}} \mathbf{D}_{q} \mathbf{P}_{q} d r d s\right] \mathbf{A}_{q}^{-1} \mathbf{C}_{q}\right\} \mathbf{T}_{g}^{\mathrm{T}} \\
& +k_{t} D_{m 33} h \mathbf{T}_{g}\left\{\mathbf{C}_{d}^{\mathrm{T}} A_{d}\left[\int_{A} P_{d} P_{d} d r d s\right] A_{d}^{-1} \mathbf{C}_{d}\right\} \mathbf{T}_{g}^{\mathrm{T}} .
\end{aligned}
$$

\subsection{Geometric Stiffness Matrix}

Shi and Voyiadjis $[134,135]$ presented a geometrically nonlinear formulation of the QCT for four-node and three node shell elements. Although their work and the present are similar that both have an explicit tangent stiffness, Shi and Voyiadjis used a von Karman assumption. The present formulation uses the full expression of the Green strain tensor. Aside from membrane stress resultants, the bending and transverse shear stress resultants are included in the geometric stiffness formulation, which make the present formulation more suitable for buckling analysis of shell structures.

Another geometrical nonlinear formulation of the QCT is by Guan and Tang [53], on a nine-node element. The geometrical stiffness matrix uses all the nonlinear strain terms, however, numerical integration is required. The work of Guam and Tang does not include material nonlinearity. On a purely geometrical nonlinear analysis, the present formulation has the advantage of being more computationally efficient as the tangent is given explicitly.

The geometric stiffness matrix is taken from the second term on the LHS of (48).

$$
\int_{\Omega}\left(\mathbf{N} \delta \Delta \mathbf{\Xi}_{m}+\mathbf{M} \delta \Delta \mathbf{\Xi}_{b}+\mathbf{Q} \delta \Delta \mathbf{\Xi}_{q}\right) d r d s
$$

The nonlinear strains are defined in (45) to (47). Equation (72) can be written in matrix form so that the quasiconforming technique can be applied. Defining the strain vector as $\boldsymbol{\eta}$ and the resultant force matrix as $\mathbf{F}_{g}$, the matrix integral form of (72) is

$$
\begin{aligned}
& \int_{\Omega}\left(\mathbf{N} \delta \Delta \boldsymbol{\Xi}_{m}+\mathbf{M} \delta \Delta \mathbf{\Xi}_{b}+\mathbf{Q} \delta \Delta \mathbf{\Xi}_{q}\right) d r d s \\
& \quad=\int_{\Omega} \delta \Delta \eta \mathbf{F}_{g} \Delta \boldsymbol{\eta} d r d s .
\end{aligned}
$$

The strain vector as $\Delta \boldsymbol{\eta}$ and the resultant force matrix as $\mathbf{F}_{g}$ are

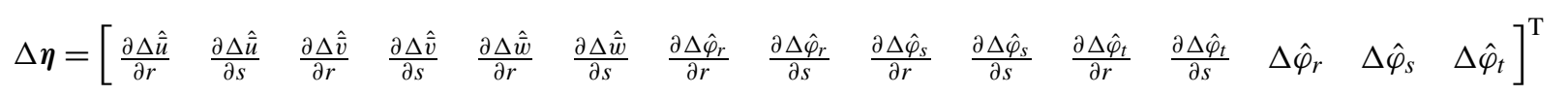

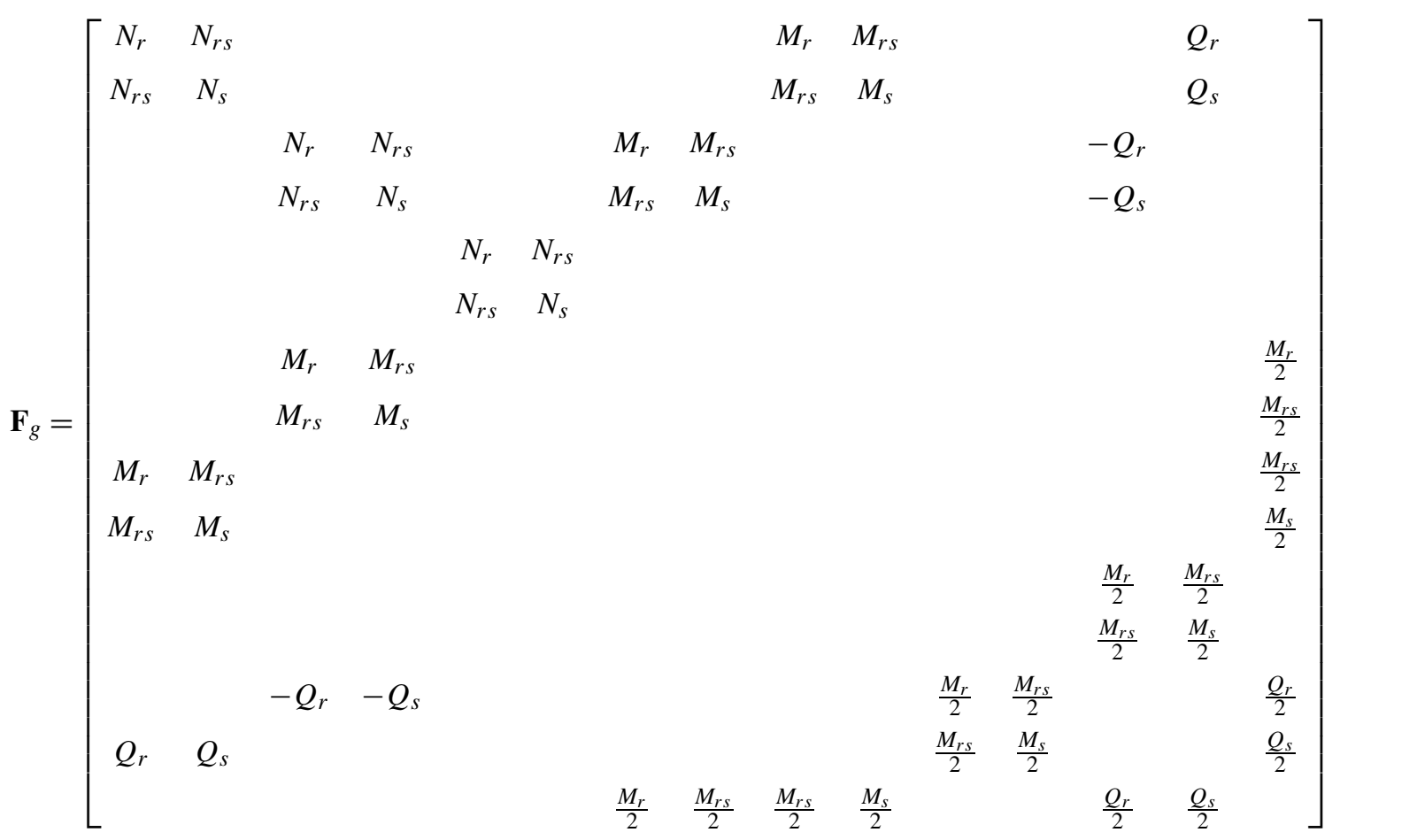


A constant strain field is assumed for the nonlinear strains.

$$
\boldsymbol{\eta}=\mathbf{P}_{g} \boldsymbol{\alpha}_{g}=\left[\begin{array}{ccccc}
1 & & & & \\
& \ldots & & & \\
& & \ldots & & \\
& & & \ldots & \\
& & & & 1
\end{array}\right]\left\{\begin{array}{c}
\alpha_{g 1} \\
\vdots \\
\vdots \\
\vdots \\
\alpha_{g 15}
\end{array}\right\} .
$$

Similar to the derivation for the linear strains, the strain vector is

$\boldsymbol{\eta}=\mathbf{B}_{g} \mathbf{T}_{g}^{\mathrm{T}}\left\{\begin{array}{c}\Delta \hat{\overline{\mathbf{U}}} \\ \Delta \hat{\boldsymbol{\theta}}\end{array}\right\}=\mathbf{P}_{g} \mathbf{A}_{g}^{-1} \mathbf{C}_{g} \mathbf{T}_{g}^{\mathrm{T}}\left\{\begin{array}{c}\Delta \hat{\overline{\mathbf{U}}} \\ \Delta \hat{\boldsymbol{\theta}}\end{array}\right\}$.

$\mathbf{A}_{\mathrm{g}}$ is a $15 \times 15$ diagonal matrix of the mid-surface area and $\mathbf{C}_{g}$ is derived using the previously given string functions. Since only a constant approximation is assumed for the strain vector, the components of $\mathbf{A}_{g}$ and $\mathbf{C}_{g}$ would be the same as some of the components of $\mathbf{A}$ and $\mathbf{C}$ for the linear strain terms. These components do not have to be re-derived or re-computed during implementation, e.g. $\mathbf{C}_{g}(1, j)=\mathbf{C}_{m}(1, j)(j=1,7,13,19)$ and $\mathbf{C}_{g}(8, j)=$ $-\mathbf{C}_{b}(5, j)(j=3-5,9-11,15-17,21-23)$.

Rows $\mathbf{C}_{g}(5, j)$ to $\mathbf{C}_{g}(7, j), \mathbf{C}_{g}(10, j)$ are solved by converting the area integral to line integrals and using (56) and (57).

$$
\begin{aligned}
\mathbf{G}_{g}(5, j)\left\{\begin{array}{c}
\Delta \hat{\overline{\mathbf{u}}} \\
\Delta \hat{\varphi}
\end{array}\right\} & =\int_{\Omega} \frac{\partial \Delta \hat{\bar{w}}}{\partial r} d r d s \\
& =\oint \Delta \hat{\bar{w}} n_{r} d L \quad(j=1,2, \ldots, 24), \\
\mathbf{G}_{g}(6, j)\left\{\begin{array}{c}
\Delta \hat{\overline{\mathbf{u}}} \\
\Delta \hat{\varphi}
\end{array}\right\} & =\int_{\Omega} \frac{\partial \Delta \hat{\bar{w}}}{\partial s} d r d s=\oint \Delta \hat{\bar{w}} n_{s} d L, \\
\mathbf{G}_{g}(7, j)\left\{\begin{array}{c}
\Delta \hat{\overline{\mathbf{u}}} \\
\Delta \hat{\varphi}
\end{array}\right\} & =\int_{\Omega} \frac{\partial \Delta \hat{\varphi}_{r}}{\partial r} d r d s=\oint \Delta \hat{\varphi}_{r} n_{r} d L \\
& =\oint\left[\Delta \varphi_{n} n_{r}-\Delta \varphi_{t} n_{s}\right] n_{r} d L,
\end{aligned}
$$$$
\mathbf{G}_{g}(10, j)\left\{\begin{array}{c}
\Delta \hat{\overline{\mathbf{u}}} \\
\Delta \hat{\varphi}
\end{array}\right\}=\int_{\Omega} \frac{\partial \Delta \hat{\varphi}_{s}}{\partial s} d r d s=\oint \Delta \hat{\varphi}_{s} n_{s} d L .
$$

$k$ indicates the side number where the line integral is being taken. $n_{r}$ and $n_{s}$ are the side normal and tangent as shown in Fig. 2. Rows 13 to 15 of $\mathbf{G}_{g}$ are determined with the following:

$$
\begin{aligned}
\mathbf{G}_{g}(13, j)\left\{\begin{array}{c}
\Delta \hat{\overline{\mathbf{u}}} \\
\Delta \hat{\varphi}
\end{array}\right\} & =\int_{\Omega} \Delta \hat{\varphi}_{r} d r d s \\
& \cong \int_{\Omega}\left[\lambda_{s} \frac{\partial \Delta \hat{\bar{w}}}{\partial s}+\left(1-\lambda_{s}\right) \Delta \hat{\varphi}_{r}\right] d r d s
\end{aligned}
$$

$$
=\lambda_{s} \oint \Delta \hat{\bar{w}} n_{s} d L+\left(1-\lambda_{s}\right) \int_{\Omega} \Delta \hat{\varphi}_{r} d r d s,
$$

$\mathbf{G}_{g}(14, j)\left\{\begin{array}{c}\Delta \hat{\overline{\mathbf{u}}} \\ \Delta \hat{\varphi}\end{array}\right\}=\int_{\Omega} \Delta \hat{\varphi}_{s} d r d s=-\lambda_{r} \oint \Delta \hat{\bar{w}} n_{r} d L$

$$
+\left(1-\lambda_{r}\right) \int_{\Omega} \Delta \hat{\varphi}_{s} d r d s,
$$

$\mathbf{G}_{g}(15, j)\left\{\begin{array}{c}\Delta \hat{\overline{\mathbf{u}}} \\ \Delta \hat{\varphi}\end{array}\right\}=\int_{\Omega} \Delta \hat{\varphi}_{t} d r d s$.

The remaining area integral is integrated by isoparametric mapping.

For a linear material analysis, the stress resultants in matrix $\mathbf{F}_{g}$ are defined as

$$
\begin{aligned}
\mathbf{N}= & \mathbf{D}_{m} \hat{\mathbf{e}}_{m}+\mathbf{D}_{m b} \hat{\mathbf{e}}_{b}=\mathbf{D}_{m} \mathbf{P}_{m} \mathbf{A}_{m}^{-1} \mathbf{G}_{m} \mathbf{T}_{g}^{\mathrm{T}}\left\{\begin{array}{c}
\hat{\overline{\mathbf{U}}} \\
\hat{\boldsymbol{\theta}}
\end{array}\right\} \\
& +\mathbf{D}_{m b} \mathbf{P}_{b} \mathbf{A}_{b}^{-1} \mathbf{G}_{b} \mathbf{T}_{g}^{\mathrm{T}}\left\{\begin{array}{c}
\hat{\hat{\mathbf{U}}} \\
\hat{\boldsymbol{\theta}}
\end{array}\right\},
\end{aligned}
$$

$$
\begin{aligned}
\mathbf{M}= & \mathbf{D}_{m b} \hat{\mathbf{e}}_{m}+\mathbf{D}_{b} \hat{\mathbf{e}}_{b}=\mathbf{D}_{m b} \mathbf{P}_{m} \mathbf{A}_{m}^{-1} \mathbf{G}_{m} \mathbf{T}_{g}^{\mathrm{T}}\left\{\begin{array}{l}
\hat{\overline{\mathbf{U}}} \\
\hat{\boldsymbol{\theta}}
\end{array}\right\} \\
& +\mathbf{D}_{b} \mathbf{P}_{b} \mathbf{A}_{b}^{-1} \mathbf{G}_{b} \mathbf{T}_{g}^{\mathrm{T}}\left\{\begin{array}{c}
\hat{\overline{\mathbf{U}}} \\
\hat{\boldsymbol{\theta}}
\end{array}\right\},
\end{aligned}
$$

$\mathbf{Q}=\mathbf{D}_{q} \hat{\mathbf{e}}_{q}=\mathbf{D}_{q} \mathbf{P}_{q} \mathbf{A}_{q}^{-1} \mathbf{G}_{q} \mathbf{T}_{g}^{\mathrm{T}}\left\{\begin{array}{c}\hat{\hat{\mathbf{U}}} \\ \hat{\boldsymbol{\theta}}\end{array}\right\}$.

The resulting geometric stiffness is

$$
\begin{aligned}
\mathbf{K}_{g} & =\mathbf{T}_{g}\left\{\mathbf{C}_{g}^{\mathrm{T}} \mathbf{A}_{g}^{-\mathrm{T}}\left[\int_{A} \mathbf{P}_{g}^{\mathrm{T}} \mathbf{F}_{g} \mathbf{P}_{g} d r d s\right] \mathbf{A}_{g}^{-1} \mathbf{C}_{g}\right\} \mathbf{T}_{g}^{\mathrm{T}} \\
& =\frac{1}{A^{2}} \mathbf{T}_{g}\left\{\mathbf{C}_{g}^{\mathrm{T}}\left[\int_{A} \mathbf{F}_{g} d r d s\right] \mathbf{C}_{g}\right\} \mathbf{T}_{g}^{\mathrm{T}} .
\end{aligned}
$$

The integral term is composed of polynomial and can be analytically solved with (15).

\subsection{Internal Force Vector}

The internal force vector is defined as the second term on the right hand side of (48).

$\int_{A}\left(\delta \Delta \mathbf{e}_{b}^{\mathrm{T}} \mathbf{M}+\delta \Delta \mathbf{e}_{m}^{\mathrm{T}} \mathbf{N}+\delta \Delta \mathbf{e}_{q}^{\mathrm{T}} \mathbf{Q}\right) d r d s$.

The resultant stresses are as defined in (80) to (82) and the linear strain terms are as previously derived. Substituting these into (84) gives the expression for the internal force vector.

$\mathbf{F}=\mathbf{T}_{g}\left\{\mathbf{C}_{m}^{\mathrm{T}} \mathbf{A}_{m}^{-\mathrm{T}}\left[\int_{\Omega} \mathbf{P}_{m}^{\mathrm{T}} \mathbf{D}_{m} \mathbf{P}_{m} d r d s\right] \mathbf{A}_{m}^{-1} \mathbf{C}_{m}\right.$ 


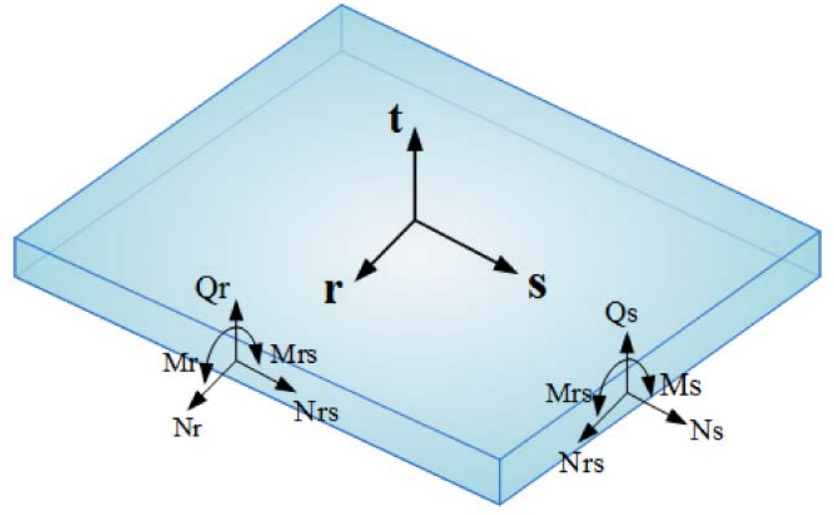

Fig. 5 Stress resultants of shell element

$$
\begin{aligned}
& +\mathbf{C}_{m}^{\mathrm{T}} \mathbf{A}_{m}^{-\mathrm{T}}\left[\int_{\Omega} \mathbf{P}_{m}^{\mathrm{T}} \mathbf{D}_{m b} \mathbf{P}_{b} d r d s\right] \mathbf{A}_{b}^{-1} \mathbf{C}_{b} \\
& +\mathbf{C}_{b}^{\mathrm{T}} \mathbf{A}_{b}^{-\mathrm{T}}\left[\int_{\Omega} \mathbf{P}_{b}^{\mathrm{T}} \mathbf{D}_{m b} \mathbf{P}_{m} d r d s\right] \mathbf{A}_{m}^{-1} \mathbf{C}_{m} \\
& +\mathbf{C}_{b}^{\mathrm{T}} \mathbf{A}_{b}^{-\mathrm{T}}\left[\int_{\Omega} \mathbf{P}_{b}^{\mathrm{T}} \mathbf{D}_{b} \mathbf{P}_{b} d r d s\right] \mathbf{A}_{b}^{-1} \mathbf{C}_{b} \\
& \left.+\mathbf{C}_{q}^{\mathrm{T}} \mathbf{A}_{q}^{-\mathrm{T}}\left[\int_{\Omega} \mathbf{P}_{q}^{\mathrm{T}} \mathbf{D}_{q} \mathbf{P}_{q} d r d s\right] \mathbf{A}_{q}^{-1} \mathbf{C}_{q}\right\} \mathbf{T}_{g}^{\mathrm{T}}\left\{\begin{array}{c}
\Delta \hat{\overline{\mathbf{U}}} \\
\Delta \hat{\boldsymbol{\theta}}
\end{array}\right\} .
\end{aligned}
$$

\subsection{Constitutive Relation}

The shell element is acted on by resultant membrane forces $\mathbf{N}$, moments $\mathbf{M}$ and transverse shear forces $\mathbf{Q}$, which are obtained by integration of stresses through the thickness.

The rigidity matrix of the reinforced concrete shell can be written in the following form:

$$
\left\{\begin{array}{c}
\Delta \mathbf{N} \\
\Delta \mathbf{M} \\
\Delta \mathbf{Q}
\end{array}\right\}=\left[\begin{array}{ccc}
\mathbf{D}_{m} & \mathbf{D}_{m b} & \mathbf{0} \\
\mathbf{D}_{m b} & \mathbf{D}_{b} & \mathbf{0} \\
\mathbf{0} & \mathbf{0} & \mathbf{D}_{q}
\end{array}\right]\left\{\begin{array}{c}
\Delta \hat{\mathbf{e}}_{m} \\
\Delta \hat{\mathbf{e}}_{b} \\
\Delta \hat{\mathbf{e}}_{q}
\end{array}\right\}=\mathbf{D}\left\{\begin{array}{c}
\Delta \hat{\mathbf{e}}_{m} \\
\Delta \hat{\mathbf{e}}_{b} \\
\Delta \hat{\mathbf{e}}_{q}
\end{array}\right\}
$$

where $\mathbf{D}_{m}, \mathbf{D}_{b}, \mathbf{D}_{m b}$ and $\mathbf{D}_{q}$ are extensile, flexural, extensileflexural and transverse shear rigidity matrices. $\mathbf{M}, \mathbf{N}$ and $\mathbf{Q}$ are the stress couple, in-plane stress and transverse shear resultant vectors in configuration $t$.

$$
\begin{aligned}
& \mathbf{M}=\left[\begin{array}{lll}
M_{r} & M_{s} & M_{r s}
\end{array}\right]^{\mathrm{T}}, \quad \mathbf{N}=\left[\begin{array}{lll}
N_{r} & N_{s} & N_{r s}
\end{array}\right]^{\mathrm{T}}, \\
& \mathbf{Q}=\left[\begin{array}{ll}
Q_{r} & Q_{s}
\end{array}\right]^{\mathrm{T}} .
\end{aligned}
$$

4 Material Model for Shell Element

\subsection{Isotropic \& Elasto-Plastic Material}

When the entire cross section is isotropic elastic, then the rigidity matrix has the following form:

$$
\begin{aligned}
& \mathbf{D}_{m}=\frac{E h}{1-v^{2}}\left[\begin{array}{ccc}
1 & v & 0 \\
v & 1 & 0 \\
0 & 0 & \frac{1-v}{2}
\end{array}\right], \\
& \mathbf{D}_{q}=\frac{5 E h}{12(1+v)}\left[\begin{array}{cc}
1 & 0 \\
0 & 1
\end{array}\right], \\
& \mathbf{D}_{b}=\frac{E h^{3}}{12\left(1-v^{2}\right)}\left[\begin{array}{ccc}
1 & v & 0 \\
v & 1 & 0 \\
0 & 0 & \frac{1-v}{2}
\end{array}\right] .
\end{aligned}
$$

The incremental constitutive law for elasto-plastic material is expressed as follows:

$$
\left\{\begin{array}{c}
\Delta \sigma_{r} \\
\Delta \sigma_{s} \\
\Delta \sigma_{r s}
\end{array}\right\}=\left[\begin{array}{lll}
C_{11} & C_{12} & C_{13} \\
C_{21} & C_{22} & C_{23} \\
C_{31} & C_{32} & C_{33}
\end{array}\right]\left\{\begin{array}{c}
\Delta \hat{e}_{r} \\
\Delta \hat{e}_{s} \\
\Delta \hat{e}_{r s}
\end{array}\right\}=\mathbf{C}^{e p} \Delta \hat{\mathbf{e}}_{m b}
$$

In this co-rotational approach the membrane-bending strain $\Delta \hat{\mathbf{e}}_{m b}$ is taken as the difference between the corotational strains in the current configuration and the corotational strain in the next configuration. This definition allows a path independent, Euler forward integration scheme, to be used. The co-rotational strain increments can be directly transformed to Cauchy stress increments without recourse to expensive transformation. The constitutive law will be based on the von Mises yield function and PrandtlReuss flow rule. For the general three-dimensional case, the Von miss yield criteria can be written in the following form:

$F=\bar{\sigma}_{e}-k \int d \varepsilon_{e}^{p}=0$.

$f$ is the yield function value, $\bar{\sigma}_{e}=\sqrt{(3 / 2) \sigma_{i j}^{\prime} \sigma_{i j}^{\prime}}$ is effective stress, $d \varepsilon_{e}^{p}$ is effective incremental plastic strain and $k \int d \varepsilon_{e}^{p}$ is the curvature of the yield surface.

Considering the increment of a specific plastic work, effective plastic strain can be shown as:

$d \varepsilon_{e}^{p}=\left[(2 / 3) d \varepsilon_{i j}^{p} d \varepsilon_{i j}^{p}\right]^{1 / 2}$

The present model is assumed to be limited to linear isotropic hardening.

In application to metal plasticity, it is often assumed that isotopic hardening is linear to the form when the yield function changes as material hardens. Letting $H$ be the hardening modulus,

$k \int d \varepsilon_{e}^{p}=\sigma_{o}+H \varepsilon_{e}^{p}$ 
At present time, the following hypothesis to calculate plastic green Lagrange strain appears to be generally accepted as the associated flow:

$d \varepsilon_{i j}^{p}=\lambda \frac{\partial f}{\partial \sigma_{i j}^{\prime}}$.

The incremental form of elastic stress and strain can be presented in following relation:

$d \sigma_{i j}=C_{i j k l}^{e}\left(d \varepsilon_{k l}-d \varepsilon_{k l}^{p}\right)$.

Where,

$C_{i j k l}=\mu^{e}\left(\delta_{i k} \delta_{j l}+\delta_{i l} \delta_{j k}\right)+\lambda^{e} \delta_{i j} \delta_{k l}$,

$\mu^{e}=\frac{E}{2(1+v)}, \quad \lambda^{e}=\frac{E v}{(1+v)(1-2 v)}$.

From the constitutive law of elasticity and concept of deviatoric and volumetric, the following can be formulated:

$d \sigma_{i j}=\lambda^{e} \delta_{i j} d \varepsilon_{k k}+2 \mu^{e} d \varepsilon_{i j}$,

$d \sigma_{i j}^{\prime}=2 \mu^{e} d \varepsilon_{i j}$.

Since, there is no contribution from volumetric stress for the plastic strain, the plastic stress itself is deviatoric. Therefore, the incremental plastic stress can be written in the following form: $C_{i j k l}^{e} d \varepsilon_{k l}^{p}=2 \mu^{e} d \varepsilon_{k l}^{p}$, thus, substituting it in the flow rule equation (92) into incremental form of constitutive relation (93).

$d \sigma_{i j}=C_{i j k l}^{e} d \varepsilon_{k l}-2 \mu^{e} \lambda \frac{\partial \bar{\sigma}_{e}}{\partial \sigma_{i j}^{\prime}}$.

When plasticity is occurring, stress direction must be satisfied. Using chain rule and consistency condition

$d f=\frac{\partial f}{\partial \sigma_{i j}^{\prime}} d \sigma_{i j}^{\prime}+\frac{\partial f}{\partial \varepsilon_{e}^{p}} d \varepsilon_{e}^{p}$, $d f=0$,

$\frac{\partial \bar{\sigma}_{e}}{\partial \sigma_{i j}^{\prime}} d \sigma_{i j}^{\prime}-\frac{\partial k}{\partial \varepsilon_{e}^{p}} d \varepsilon_{e}^{p}=0$.

Substituting $d \varepsilon_{e}^{p}=\left[(2 / 3) d \varepsilon_{i j}^{p} d \varepsilon_{i j}^{p}\right]^{1 / 2}, d \sigma_{i j}^{\prime}=2 \mu^{e} d \varepsilon_{i j}^{e}$ and the flow rule equation (92) into consistency condition, (98).

$\frac{\partial \bar{\sigma}_{e}}{\partial \sigma_{i j}^{\prime}} 2 \mu^{e}\left(d \varepsilon_{i j}-d \varepsilon_{i j}^{p}\right)-\frac{\partial k}{\partial \varepsilon_{e}^{p}}\left(\frac{2}{3} d \varepsilon_{i j}^{p} d \varepsilon_{i j}^{p}\right)^{\frac{1}{2}}=0$,

$\frac{\partial \bar{\sigma}_{e}}{\partial \sigma_{i j}^{\prime}} 2 \mu^{e}\left(d \varepsilon_{i j}-\lambda \frac{\partial f}{\partial \sigma_{i j}^{\prime}}\right)-\lambda \frac{\partial k}{\partial \varepsilon_{e}^{p}}\left(\frac{2}{3} \frac{\partial f}{\partial \sigma_{i j}^{\prime}} \frac{\partial f}{\partial \sigma_{i j}^{\prime}}\right)^{\frac{1}{2}}=0$,

$H=\frac{d k}{d \varepsilon_{e}^{p}}\left(\frac{2}{3} \frac{\partial f}{\partial \sigma_{i j}^{\prime}} \frac{\partial f}{\partial \sigma_{i j}^{\prime}}\right)^{\frac{1}{2}}=\frac{d k}{d \varepsilon_{e}^{p}}$,

$\frac{\partial \bar{\sigma}_{e}}{\partial \sigma_{i j}^{\prime}} 2 \mu^{e}\left(d \varepsilon_{i j}-\lambda \frac{\partial f}{\partial \sigma_{i j}^{\prime}}\right)-H \lambda=0$.

Substituting the following relation $\frac{\partial f}{\partial \sigma_{i j}^{\prime}}=\frac{\partial \bar{\sigma}_{e}}{\partial \sigma_{i j}^{\prime}}=\frac{3 \sigma_{i j}^{\prime}}{2 \bar{\sigma}_{e}}$ into (99), the factor in flow equation can be calculated as follow:

$\lambda=\frac{3 \mu}{\bar{\sigma}_{e}(H+3 \mu)} \sigma_{i j}^{\prime} d \varepsilon_{i j}$.

Then, substituting $\lambda$ into elastic stress strain relation (96)

$d \sigma_{i j}=\left(C_{i j k l}^{e}-\frac{6 \mu^{e 2} \sigma_{k l} \frac{\partial \bar{\sigma}_{e}}{\partial \sigma_{i j}^{\prime}}}{\bar{\sigma}_{e}\left(H+3 \mu^{e}\right)}\right) d \varepsilon_{k l}=C_{i j k l}^{e f} d \varepsilon_{k l}$.

Elasto-plastic tangent modules can be formulated as follow:

$\mathbf{C}^{e p}=\mathbf{C}^{e}-\frac{9 \mu^{2}}{(3 \mu+H) \bar{\sigma}_{e}^{2}} \boldsymbol{\sigma}^{\prime} \otimes \boldsymbol{\sigma}^{\prime}$.

Where, $\sigma^{\prime} \otimes \sigma^{\prime}=\sigma_{I J}^{\prime} \sigma_{K L}^{\prime}$.

$\mathbf{C}^{e p}=\frac{E}{1+v}\left[\begin{array}{cccccc}\frac{1-v}{1-2 v}-\frac{3 \kappa \sigma_{x}^{\prime 2}}{2 \sigma_{y}^{2}} & \frac{v}{1-2 v}-\frac{3 \kappa \sigma_{x}^{\prime} \sigma_{y}^{\prime}}{2 \sigma_{y}^{2}} & -\frac{3 \kappa \sigma_{x}^{\prime} \sigma_{x y}^{\prime}}{2 \sigma_{y}^{2}} & \ldots & \ldots & \frac{v}{1-2 v}-\frac{3 \kappa \sigma_{x}^{\prime} \sigma_{z}^{\prime}}{2 \sigma_{y}^{2}} \\ \frac{v}{1-2 v}-\frac{3 \kappa \sigma_{y}^{\prime} \sigma_{x}^{\prime}}{2 \sigma_{y}^{2}} & \frac{1-v}{1-2 v}-\frac{3 \kappa \sigma_{y}^{\prime 2}}{2 \sigma_{y}^{2}} & -\frac{3 \kappa \sigma_{y}^{\prime} \sigma_{x y}^{\prime}}{2 \sigma_{y}^{2}} & \ldots & \ldots & \frac{v}{1-2 v}-\frac{3 \kappa \sigma_{y}^{\prime} \sigma_{z}^{\prime}}{2 \sigma_{y}^{2}} \\ -\frac{3 \kappa \sigma_{x y}^{\prime} \sigma_{x}^{\prime}}{2 \sigma_{y}^{2}} & -\frac{3 \kappa \sigma_{x y}^{\prime} \sigma_{y}^{\prime}}{2 \sigma_{y}^{2}} & \frac{1}{2}-\frac{3 \kappa \sigma_{x y}^{\prime 2}}{2 \sigma_{y}^{2}} & \ldots & \ldots & -\frac{3 \kappa \sigma_{x y}^{\prime} \sigma_{z}^{\prime}}{2 \sigma_{y}^{2}} \\ \ldots & \ldots & \ldots & \ldots & \ldots & \ldots \\ \ldots & \ldots & \ldots & \ldots & \ldots & \ldots \\ \frac{v}{1-2 v}-\frac{3 \kappa \sigma_{z}^{\prime} \sigma_{x}^{\prime}}{2 \sigma_{y}^{2}} & \frac{v}{1-2 v}-\frac{3 \kappa \sigma_{z}^{\prime} \sigma_{y}^{\prime}}{2 \sigma_{y}^{2}} & -\frac{3 \kappa \sigma_{z}^{\prime} \sigma_{x y}^{\prime}}{2 \sigma_{y}^{2}} & \ldots & \ldots & \frac{1-v}{1-2 v}-\frac{3 \kappa \sigma_{z}^{\prime 2}}{2 \sigma_{y}^{2}}\end{array}\right]$. 
Fig. 6 Laminate geometry: (a)

NL layered laminate; (b)

Lamina orientation; $r 1$ and $s 1$ are rotated axes

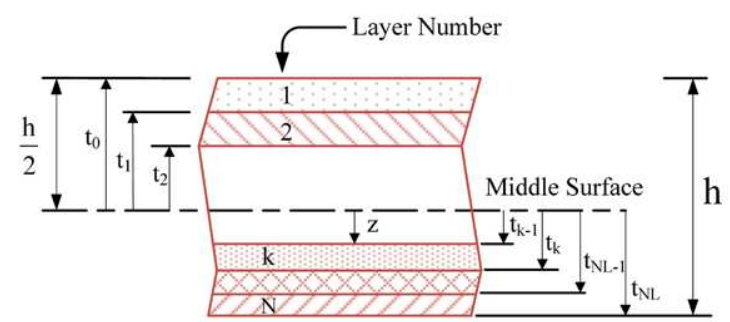

a)

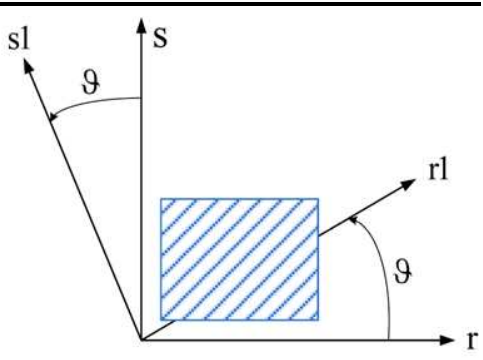

b)
Where, $\kappa=\frac{3 \mu^{e}}{3 \mu^{e}+H}$.

All above modifications are valid for 3D and 2D plane strain condition; therefore, it is necessary to convert all above constitutive relations to plane stress conditions in order to use them in shell analysis. For plane stress analysis, the following formula can be used:

$\mathbf{C}^{e p}=\left[\begin{array}{lll}C_{1}-C_{31} \frac{C_{31}}{C_{36}} & C_{7}-C_{31} \frac{C_{32}}{C_{36}} & C_{13}-C_{31} \frac{C_{33}}{C_{36}} \\ C_{2}-C_{32} \frac{C_{31}}{C_{36}} & C_{8}-C_{32} \frac{C_{32}}{C_{36}} & C_{14}-C_{32} \frac{C_{33}}{C_{36}} \\ C_{3}-C_{33} \frac{C_{31}}{C_{36}} & C_{9}-C_{33} \frac{C_{32}}{C_{36}} & C_{15}-C_{33} \frac{C_{33}}{C_{36}}\end{array}\right]$.

$C_{i}$ in (104) is the column wise representation of matrix in (103), where $i$ varies from 1 to 36 .

Since the elasto-plastic strain increments $\Delta \boldsymbol{\varepsilon}$ are finite, the calculated stress state at the end of the current step will not lie on the yield surface. However, by dividing $\Delta e$ into a suitable number of sub increments, and applying incremental stress strain relationship, the error can be greatly reduced. During this process, there will be a tendency for the stress state to drift away from the yield surface, but this effect can easily be eliminated by moving back to the yield surface at the end of each sub incremental step. For this purpose, the so called "normal return" method can be used, i.e.

$\sigma_{i j}=\alpha \sigma_{i j}$

Coefficient $\alpha$ can be calculated using yield function and returning to yield surface tangentially from the current state, $\alpha=1-(3 \mu f) /\left[(3 \mu+K) \bar{\sigma}_{e}\right]$.

Because sub incremental calculation is expensive, it is recommended that the number of sub increments to be used in any given case, should be calculated automatically. In this respect, it is worth to calculate the number of sub increments $n$ purely on the excess stress.

$n=20 \sqrt{f_{n+1}^{\text {trial }}} / \sigma_{y}+1$.

Here, yield function $f$ has been calculated using square of effective stress $\bar{\sigma}_{e}^{2}$.

$\gamma=(1-r) / n$.
The incremental strain in sub increments $\Delta \varepsilon_{i j s}^{\text {total }}=\gamma \Delta \varepsilon_{i j}^{\text {total }}$. Here, $r$ represents the amount of elastic strain undergone when stress state at the transition of elastic to plastic. The incremental stress resultants $(\Delta \mathbf{N}, \Delta \mathbf{M})$ and strains $\Delta \hat{\mathrm{e}}_{m}, \Delta \hat{\mathrm{e}}_{b}$ are related by elasto-plastic rigidity in the membranebending matrix $D_{e p}$.

$$
\begin{aligned}
\left\{\begin{array}{c}
\Delta \mathbf{N} \\
\Delta \mathbf{M}
\end{array}\right\} & =\left[\begin{array}{cc}
\int_{-2 / h}^{2 / h} \mathbf{C}^{e p} d t & \int_{-2 / h}^{2 / h} t \mathbf{C}^{e p} d t \\
\int_{-2 / h}^{2 / h} t \mathbf{C}^{e p} d t & \int_{-2 / h}^{2 / h} t^{2} \mathbf{C}^{e p} d t
\end{array}\right]\left\{\begin{array}{c}
\Delta \hat{\mathbf{e}}_{m} \\
\Delta \hat{\mathbf{e}}_{b}
\end{array}\right\} \\
& =\left[\begin{array}{cc}
\mathbf{D}_{m} & \mathbf{D}_{m b} \\
\mathbf{D}_{m b} & \mathbf{D}_{b}
\end{array}\right]=\mathbf{D}^{e p}\left\{\begin{array}{c}
\Delta \hat{\mathbf{e}}_{m} \\
\Delta \hat{\mathbf{e}}_{b}
\end{array}\right\} .
\end{aligned}
$$

\subsection{Laminate Composite Constitutive Relation}

The anisotropic stress-strain relationship of orthotropic laminated composites for the $k$ th layer, shown in Fig. 6a, follows expressions found in most composite texts. The orientation of the principal material coordinates is referred from the element local coordinates $(r, s)$, shown in Fig. $6 \mathrm{~b}$.

By integrating the stresses through the thickness, the resultant forces $\mathbf{N}=\left[N_{r} N_{s} N_{r s}\right]^{\mathrm{T}}$, moments $\mathbf{M}=$ $\left[\begin{array}{lll}M_{r} & M_{s} & M_{r s}\end{array}\right]^{\mathrm{T}}$ and transverse shear forces $\mathbf{Q}=\left[\begin{array}{ll}Q_{r} & Q_{s}\end{array}\right]^{\mathrm{T}}$ are obtained. The compact incremental constitutive relations of the composite laminate is

$$
\left\{\begin{array}{c}
\Delta \mathbf{N} \\
\Delta \mathbf{M} \\
\Delta \mathbf{Q}
\end{array}\right\}=\left[\begin{array}{ccc}
\mathbf{D}_{m} & \mathbf{D}_{m b} & \\
\mathbf{D}_{m b} & \mathbf{D}_{b} & \\
& & \mathbf{D}_{q}
\end{array}\right]\left\{\begin{array}{c}
\Delta \mathbf{e}_{m} \\
\Delta \mathbf{e}_{b} \\
\Delta \mathbf{e}_{q}
\end{array}\right\}
$$

where

$$
\begin{aligned}
& \mathbf{D}_{m}=\sum_{k=1}^{N L} \bar{C}_{i j}^{k}\left(t_{k-1}-t_{k}\right), \\
& \mathbf{D}_{m b}=\frac{1}{2} \sum_{k=1}^{N L} \bar{C}_{i j}^{k}\left(t_{k-1}^{2}-t_{k}^{2}\right),
\end{aligned}
$$

$\mathbf{D}_{b}=\frac{1}{3} \sum_{k=1}^{N L} \bar{C}_{i j}^{k}\left(t_{k-1}^{3}-t_{k}^{3}\right) \quad$ for $i, j=1,2,3$. 
$\bar{C}_{i j}^{k}$ is the membrane-bending stiffness and $N L$ is the total number of layers.

In the case of composite materials, there is a need to adequately define the effective transverse shear stiffness for a wide range of material properties and laminate geometry. In conventional formulation, the transverse shear forces are obtained by integration through the laminate thickness. The integration expression is

$$
\mathbf{Q}=\kappa^{q} \sum_{k=1}^{N L} \bar{C}_{i j}^{k}\left(t_{k-1}-t_{k}\right) e_{q}=\kappa^{q} \mathbf{D}_{q} \mathbf{e}_{q} \quad \text { for } i, j=4,5 \text {. }
$$

In this case, the shear stiffness $\mathbf{D}_{q}$ is too large to deal with the transverse shear response. Reissner's value of $\kappa^{q}=5 / 6$ is used as the transverse shear correction factor in finite element formulations. Using $\kappa^{q}$ would give reasonable results. However, stresses in equivalent-single layer theory are discontinuous at each layer interface. Then, thick laminated plates and shells based on the Mindlin-Reissner theory will give inaccurate solutions for stresses. For this reason, an improved transverse shear stiffness that also gives accurate shear stresses is required.

The basic idea in calculating the transverse shear stresses is to obtain it from the transverse shear forces, by neglecting the influence of the membrane forces and assuming two cylindrical bending modes, Rolfes and Rohwer [123]. The equilibrium forces in $\mathrm{r}$ and $\mathrm{s}$ direction, solved with respect to the transverse shear stresses, is

$\boldsymbol{\sigma}_{t}=\left\{\begin{array}{l}\sigma_{r t} \\ \sigma_{s t}\end{array}\right\}^{k}=-\int_{-h / 2}^{h / 2}\left\{\begin{array}{l}\sigma_{r, r}^{k}+\sigma_{r s, s}^{k} \\ \sigma_{s, s}^{k}+\sigma_{r s, r}^{k}\end{array}\right\} d t$.

Using the material law for the $k$ th lamina,

$\boldsymbol{\sigma}_{m}=\left\{\begin{array}{c}\sigma_{r} \\ \sigma_{s} \\ \tau_{r s}\end{array}\right\}=\overline{\mathbf{C}}^{k}\left(\boldsymbol{\varepsilon}^{0}+t \boldsymbol{\kappa}\right)$

where $\overline{\mathbf{C}}^{k}$ is the reduced stiffness of the $k$ th lamina, and $\boldsymbol{\varepsilon}^{0}$ and $\boldsymbol{\kappa}$ denote the laminate strains and curvatures, respectively, yields

$$
\begin{aligned}
\sigma_{t}= & -\int_{-h / 2}^{-h / 2+t}\left\{\left[\begin{array}{lll}
\bar{C}_{11} & \bar{C}_{12} & \bar{C}_{16} \\
\bar{C}_{16} & \bar{C}_{26} & \bar{C}_{66}
\end{array}\right]^{k}\left(\varepsilon_{, r}^{0}+t \kappa_{, r}\right)\right. \\
& \left.+\left[\begin{array}{lll}
\bar{C}_{16} & \bar{C}_{26} & \bar{C}_{66} \\
\bar{C}_{12} & \bar{C}_{22} & \bar{C}_{26}
\end{array}\right]^{k}\left(\varepsilon_{, s}^{0}+t \kappa_{, s}\right)\right\} d t .
\end{aligned}
$$

The laminate membrane strain can be expressed by the moments if the membrane forces $\mathbf{N}$ are neglected. This assumption may be applicable since the influence of the membrane forces on the transverse shear stresses is very small. Then, by the laminate elastic relation for $\mathbf{N}$,

$$
\begin{gathered}
\mathbf{N}=\mathbf{0}=\mathbf{D}_{m} \boldsymbol{\varepsilon}^{0}+\mathbf{D}_{m b} \boldsymbol{\kappa}, \\
\boldsymbol{\varepsilon}^{0}=-\mathbf{D}_{m}^{-1} \mathbf{D}_{m b} \boldsymbol{\kappa} .
\end{gathered}
$$

Substitution of $\boldsymbol{\varepsilon}^{0}$ in (115) into the laminate elastic relation for $\mathbf{M}$ solves for $\boldsymbol{\kappa}$.

$$
\begin{gathered}
\mathbf{M}=\mathbf{D}_{m b}^{\mathrm{T}} \boldsymbol{\varepsilon}^{0}+\mathbf{D}_{b} \boldsymbol{\kappa}=\mathbf{D}_{m b}^{\mathrm{T}}\left[-\mathbf{D}_{m}^{-1} \mathbf{D}_{m b} \boldsymbol{\kappa}\right]+\mathbf{D}_{b} \boldsymbol{\kappa}, \\
\boldsymbol{\kappa}=\left[\mathbf{D}_{b}-\mathbf{D}_{m b}^{\mathrm{T}} \mathbf{D}_{m}^{-1} \mathbf{D}_{m b}\right] \mathbf{M}=\widehat{\mathbf{D}}^{-1} \mathbf{M} .
\end{gathered}
$$

Substituting (115) and (116) into the equilibrium condition in (114), provides transverse shear stresses depending only on the moment derivatives with respect to $r$ and $s$.

$$
\begin{aligned}
\boldsymbol{\sigma}_{t}= & -\int_{-h / 2}^{-h / 2+t}\left[\begin{array}{lll}
\bar{C}_{11} & \bar{C}_{12} & \bar{C}_{16} \\
\bar{C}_{16} & \bar{C}_{26} & \bar{C}_{66}
\end{array}\right]^{k}[\overline{\mathbf{A B D}}] \mathbf{M}_{, r} d t \\
& -\int_{-h / 2}^{-h / 2+t}\left[\begin{array}{lll}
\bar{C}_{16} & \bar{C}_{26} & \bar{C}_{66} \\
\bar{C}_{12} & \bar{C}_{22} & \bar{C}_{26}
\end{array}\right]^{k}[\overline{\mathbf{A B D}}] \mathbf{M}_{, s} d t
\end{aligned}
$$

where

$\overline{\mathbf{A B D}}=\left[-\mathbf{D}_{m}^{-1} \mathbf{D}_{m b}+t\right] \widehat{\mathbf{D}}^{-1}$

By using a cylindrical bending assumption, (117) can be reduced further as seen in the following equations. Assuming cylindrical bending about the $r$-axis and $s$-axis yields, respectively,

$\mathbf{M}_{, r}=\left\{\begin{array}{c}M_{r, r} \\ 0 \\ 0\end{array}\right\} \quad$ and $\quad \mathbf{M}_{, s}=\left\{\begin{array}{c}0 \\ M_{s, s} \\ 0\end{array}\right\}$

The derivative of the moments can be related to the shear forces via

$Q_{r}=M_{r, r} \quad$ and $\quad Q_{s}=M_{s, s}$.

Which finally results in a simpler expression for the transverse shear stresses

$$
\begin{aligned}
\boldsymbol{\sigma}_{t}= & -\int_{-h / 2}^{-h / 2+t}\left[\begin{array}{lll}
\bar{C}_{11} & \bar{C}_{12} & \bar{C}_{16} \\
\bar{C}_{16} & \bar{C}_{26} & \bar{C}_{66}
\end{array}\right]^{k}[\overline{\mathbf{A B D}}]\left\{\begin{array}{c}
Q_{r} \\
0 \\
0
\end{array}\right\} d t \\
& -\int_{-h / 2}^{+h / 2+t}\left[\begin{array}{llll}
\bar{C}_{16} & \bar{C}_{26} & \bar{C}_{66} \\
\bar{C}_{12} & \bar{C}_{22} & \bar{C}_{26}
\end{array}\right]^{(k)}[\overline{\mathbf{A B D}}]\left\{\begin{array}{c}
0 \\
Q_{s} \\
0
\end{array}\right\} d t,
\end{aligned}
$$

$\boldsymbol{\sigma}_{t}=\left[\begin{array}{ll}H_{11} & H_{12} \\ H_{21} & H_{22}\end{array}\right]\left[\begin{array}{l}Q_{r} \\ Q_{s}\end{array}\right] \quad$ or $\quad \boldsymbol{\sigma}_{t}=\mathbf{h}(t) \mathbf{Q}$. 
The effective laminate stiffness matrix can be obtained through the transverse shear strain energy and is expressed as follow:

$\mathbf{D}_{q}=\left[\int_{-h / 2}^{+h / 2} \mathbf{h}^{\mathrm{T}} \mathbf{G}^{-1} \mathbf{h} d t\right]^{-1}$

where $\mathbf{G}$ is the matrix of transverse shear modulus.

\subsection{Laminate Composite Progressive Failure Analysis}

\subsubsection{Failure Criteria}

Four failure criteria for single fiber reinforced composite layer under in-plane loading are considered, (1) Maximum Stress Criterion, (2) Tsai-Hill Criterion, (3) Tsai-Wu Criterion and (4) Modified Puck Criterion. These criteria may be used to check for first ply failure of composite structures or in determining ultimate loads for complete laminate failure.

The maximum stress failure criterion assumes that failure occurs whenever any one component of stress attains its limiting value, independent of the values of all other components of stress. The "safe" condition for the maximum stress failure criterion can be written mathematically in the form

$$
\begin{array}{lc}
X_{c}<\sigma_{1}<X_{t}, & \left|\tau_{23}\right|<Q_{A}, \\
Y_{c}<\sigma_{2}<Y_{t}, & \left|\tau_{31}\right|<R_{A}, \\
Z_{c}<\sigma_{3}<Z_{t}, & \left|\tau_{12}\right|<S_{A} .
\end{array}
$$

The Tsai-Hill theory (1968) [159] represents an attempt to apply Hill's [60] anisotropic plasticity theory to failure of homogeneous, anisotropic materials. For the case of plane stress in the $1-2$ plane (i.e. $\sigma_{3}=\tau_{13}=\tau_{23}=0$ ) of a transversely isotropic material $(Y=Z)$, the Tsai-Hill failure criterion is

$$
\frac{\sigma_{1}^{2}}{X^{2}}-\frac{\sigma_{1} \sigma_{2}}{X^{2}}+\frac{\sigma_{2}^{2}}{Y^{2}}+\frac{\tau_{12}^{2}}{S_{A}^{2}}=1 .
$$

In the Tsai-Wu criterion a complete quadratic tensor polynomial with linear terms is included. It incorporates tensile and compressive strengths in one expression and provides more comprehensive interaction terms. For planar states of stress, the tensor polynomial failure criteria is

$$
\begin{aligned}
F\left(\sigma_{i}\right) & =F_{1} \sigma_{1}+F_{2} \sigma_{2}+F_{11} \sigma_{12}+F_{22} \sigma_{22}+F_{66} \sigma_{62} \\
& =1
\end{aligned}
$$

with

$$
\begin{array}{ll}
F_{1}=\frac{1}{X_{T}}+\frac{1}{X_{C}}, & F_{2}=\frac{1}{Y_{T}}+\frac{1}{Y_{C}}, \\
F_{11}=-\frac{1}{X_{T} X_{C}}, & F_{22}=-\frac{1}{Y_{T} Y_{C}}, \quad F_{66}=\frac{1}{S_{A}^{2}} .
\end{array}
$$

In the Modified Puck Criterion, it is assumed that fiber failures are determined by $\sigma_{1}$ and matrix failures are determined by $\sigma_{2}$ and $\sigma_{6}$. This criterion combines simple computation with simple testing allowing interaction of stresses in both tension and compression, although it allows fewer interactions than the Tsai-Wu criterion.

$\sigma_{1}=X_{t}$ or $X_{c}$,

$\frac{\sigma_{2}^{2}}{Y_{t} Y_{c}}+\sigma_{2}\left(\frac{1}{Y_{t}}+\frac{1}{Y_{c}}\right)+\frac{\sigma_{6}^{2}}{Q_{A}^{2}}=1$

\subsubsection{General Procedure of Composite Failure Analysis}

In general, an anisotropic laminate may be subjected to a combination of different types of loading. Because of this, many experiments and mathematical formulations have been done to develop different failure criteria, but due to the complex stress inter actions within the wide range of fiber and matrix material configurations; none may be applied universally with complete confidence. The choice of criterion will usually be based on what strength data are available. A general procedure of composite failure analysis, reference may also be made in Reddy et al. $[117,121]$ is as follows:

1. After nonlinear iterative displacement convergence is achieved, stresses in each layer of each element are computed.

2. The stresses are then transformed to the principal material coordinates and checked against a chosen failure criterion.

3. When layer failure occurs, the laminate stiffness is recomputed and nonlinear analysis is again performed with the same load magnitude.

4. When no failure occurs, the load is incremented.

5. Steps 1 to 4 are repeated until complete failure has occurred or the maximum number of iterations has been reached.

A single layer criterion may be used to perform step-by-step analysis of the whole laminate. These criteria may be used in checking for first ply failure of composite structures or in determining ultimate loads of complete laminate failure. The following assumptions are made during layer failure in a load step:

1. Elements in a structure have independent stiffness. When failure in an element occurs, only the stiffness of this element is updated.

2. When failure has occurred based on the Tsai-Wu, TsaiHill or maximum stress criterion, only the property that relates to the dominant failure mode is reduced to zero. Thus, $\sigma 1$ corresponds to $E 1, \sigma 2$ to $E 2$, and $\sigma 6$ to $G 12$. 
3. For the modified Puck criterion, fiber direction failure would correspond to complete layer failure because the fiber strength is assumed to be much greater than the matrix strength. Matrix failure however would reduce only $E 2$ and $G 12$.

4. Since the improved transverse shear stiffness based on FSDT is used, the transverse shear stiffness is only updated when a layer completely fails. This assumption is acceptable and consistent with the second assumption since transverse shear stresses are relatively much smaller compared to the other in-plane stresses and thus would not be dominating failure. This would only be limited, if the loads were direct transverse shear loads.

5. When a layer fails, it is assumed that the thickness of the failed layer is unchanged.

\subsection{Reinforced Concrete Material}

The shell thickness is divided into a series of plain (unreinforced) concrete layers and of reinforcing steel layers. Plain concrete layers can either be elastic, (singly or doubly) cracked, and yielded or crushed. An appropriate stress-strain relation is used for each of these states of behaviour. On the other hand, the reinforcing steel is replaced by an equivalent smeared uniformly distributed steel layer with stiffness only in the direction of the reinforcement and the bilinear stressstrain relationship is assumed. The equivalent thickness of the steel layer is determined such that the corresponding area of reinforcement in the layer remains unchanged. Ordinarily, a concrete shell is reinforced by at least two sets of reinforcing bars. It is also assumed that the reinforcing steel is arranged in layers forming grids intersecting each other at arbitrary angles. Any number of such layers can be accounted for and each layer is to be located exactly in space for the purpose of generating its stiffness properties. Perfect bond is assumed to exist between the reinforcing steel and the surrounding concrete. The tension stiffening and dowel action are included to take into account the interaction between concrete and steel.

\subsubsection{Elasto-Plasticity-Based Model in Compressive Behavior of Concrete}

Based on the flow theory of plasticity, the nonlinear compressive behavior of concrete is characterized by a yield condition, i.e. the flow and hardening rules and the crushing condition.

The Yield Condition In the present study of reinforced concrete plates and shells, the following yield condition in terms of the first two stress invariants is employed [61].

$f\left(I_{1}, J_{2}\right)=\left[\beta\left(3 J_{2}\right)+\alpha I_{1}\right]^{1 / 2}=\sigma_{0}$.

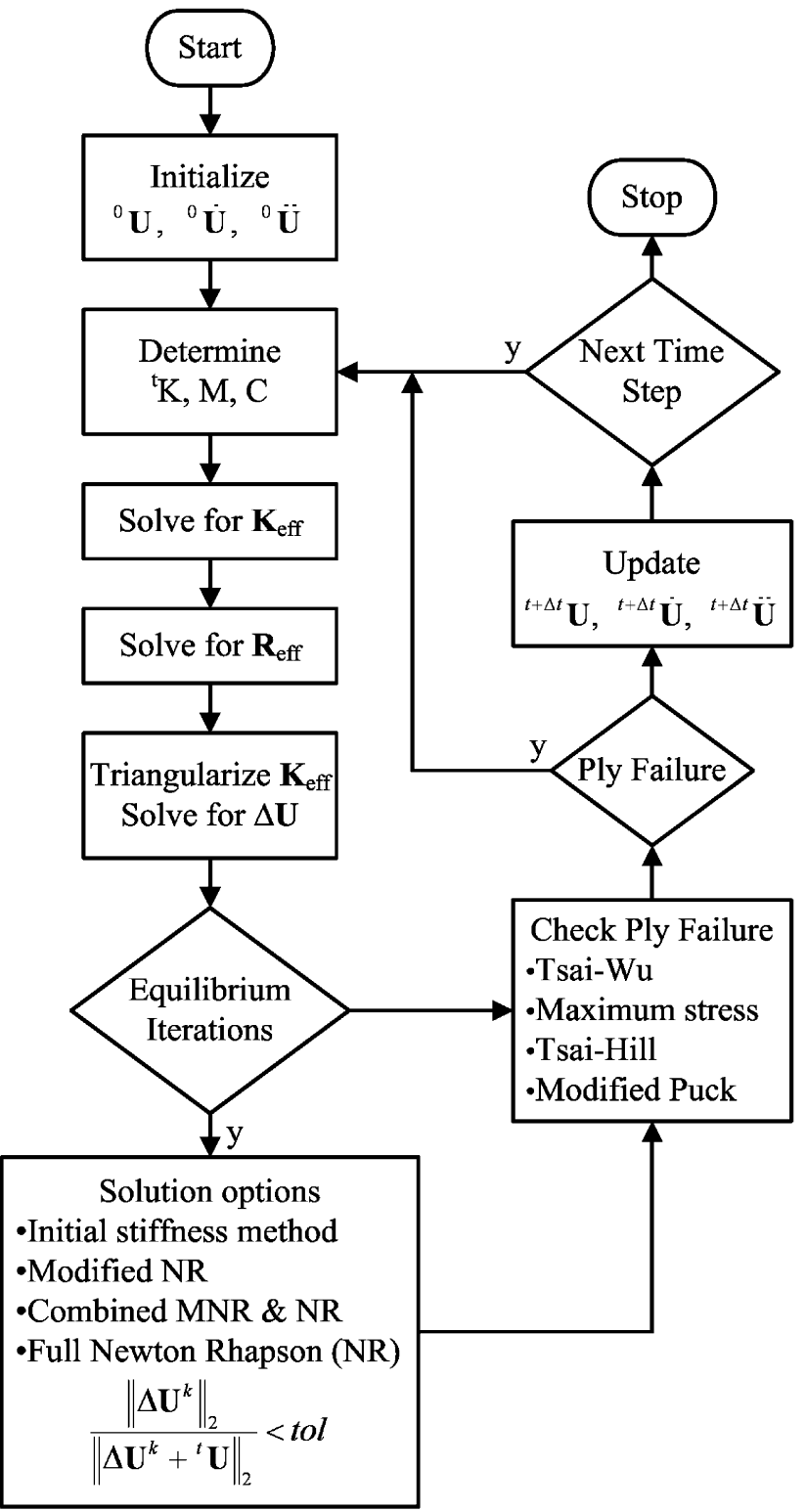

Fig. 7 Flow chart of non-linear dynamic analysis with progressive ply failure

In terms of principal stresses, the expression for yielding can be written as

$$
\begin{aligned}
\beta & {\left[\left(\sigma_{1}^{2}+\sigma_{2}^{2}+\sigma_{3}^{2}\right)-\left(\sigma_{1} \sigma_{2}+\sigma_{1} \sigma_{3}+\sigma_{1} \sigma_{3}\right)\right] } \\
& \quad+\alpha\left(\sigma_{1}+\sigma_{2}+\sigma_{3}\right) \\
= & \left(\sigma_{0}\right)^{2}
\end{aligned}
$$

$\alpha$ and $\beta$ are material parameters. $\sigma_{0}$ is the equivalent effective stress, taken as the compressive stress $f_{c}^{\prime}$ from a uniaxial test. $f_{c}^{\prime}$ can be considered as the most reliable constant to characterize the concrete behaviour and is easily obtainable. The yield condition is then a function of only one material parameter $\left(\sigma_{0}=f_{c}^{\prime}\right)$. For practical proposes, a relation can 
Fig. 8 The layered model of reinforced concrete shell element

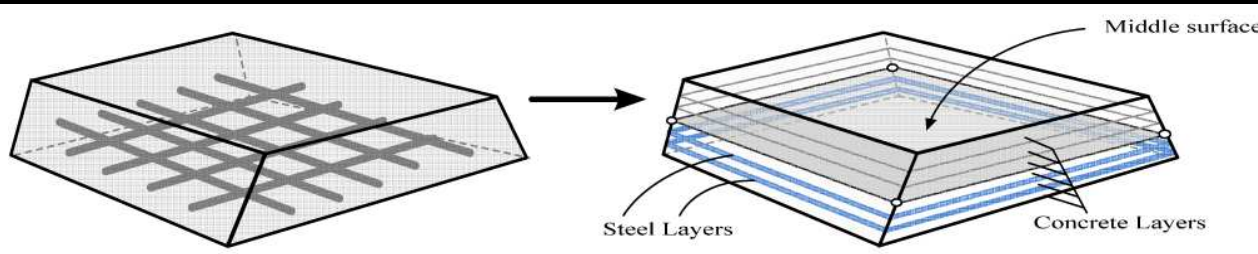

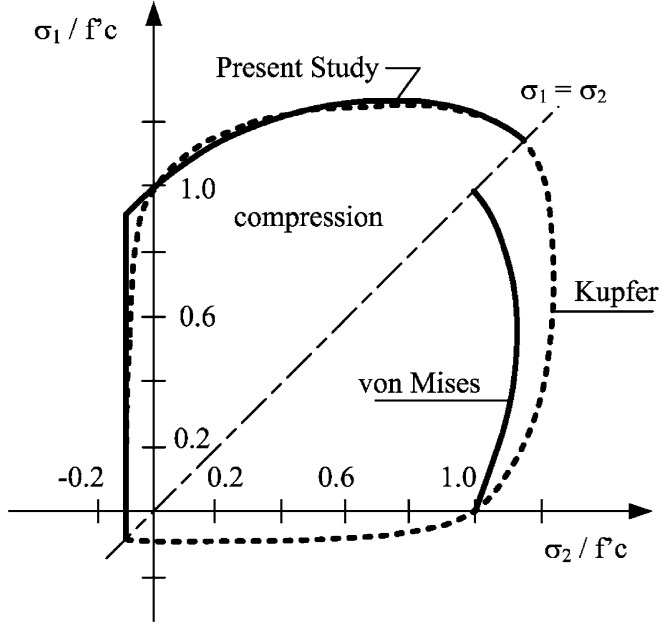

Fig. 9 Biaxial compressive and tensile cracking criterion for concrete

be assumed between the equal biaxial yield strength, $f_{c b}$, and the uniaxial yield strength, $f_{c}^{\prime}$.

$f_{c b}=1.16$ to $1.20 f_{c}^{\prime}$.

If Kupfer's results [150] are employed $\left(f_{c b}=1.16 f_{c}^{\prime}\right)$, the material constants are

$\alpha=0.355 \sigma_{0}$,

$\beta=1.355$

and the yield condition can be written in term of the stress component as:

$$
\begin{aligned}
& {\left[1.355\left[\left(\sigma_{r}^{2}+\sigma_{s}^{2}-\sigma_{r} \sigma_{s}\right)+3\left(\tau_{r s}^{2}+\tau_{r t}^{2}+\tau_{s t}^{2}\right)\right]\right.} \\
& \left.\quad+0.355 \sigma_{0}\left(\sigma_{r}+\sigma_{s}\right)\right]^{1 / 2} \\
& \quad=\sigma_{0} .
\end{aligned}
$$

This expression is compared with the experimental results of Kupfer et al. [83] in the biaxial stress space, Fig. 9. Both perfect plastic and strain-hardening plasticity approaches are employed, which are illustrated for one-dimension in Fig. 10.

The Flow Rule Since reliable data for concrete under biaxial loading is not available, except the data from Kupfer et al. [83], the normality of the plasticity deformation rate vector to the yield surface is assumed. This only requires the derivative of the yield surface. The plastic strain increment is defined as

$d \varepsilon_{i j}^{p}=d \kappa \frac{\partial f(\sigma)}{\partial \sigma_{i j}}$.

$d \kappa$ is a proportionality constant which determines the magnitude of the plastic strain increment, and the gradient $d f(\sigma) / d \sigma_{i j}$ defines its direction to be perpendicular to the yield surface. The current stress function $f(\sigma)$ is the yield condition; or the subsequent loading functions in the strainhardening model.

The yield function derivatives, which define the flow vector $(a)$, take the following explicit expressions for the yield surface:

$a^{\mathrm{T}}=\left[a_{1}, a_{2}, a_{3}, a_{4}, a_{5}\right]^{\mathrm{T}}=\left[\frac{\partial f}{\partial \sigma_{r}}, \frac{\partial f}{\partial \sigma_{s}}, \frac{\partial f}{\partial \tau_{r s}}, \frac{\partial f}{\partial \tau_{r t}}, \frac{\partial f}{\partial \tau_{s t}}\right]^{\mathrm{T}}$,

$a_{1}=\frac{\left[2\left(c^{2}+\beta\right) \sigma_{r}+\left(2 c^{2}-\beta\right) \sigma_{s}\right]}{\bar{C}}+c$,

$a_{2}=\frac{\left[2\left(c^{2}+\beta\right) \sigma_{s}+\left(2 c^{2}-\beta\right) \sigma_{r}\right]}{\bar{C}}+c$

$a_{3}=\frac{6 \beta \tau_{r s}}{\bar{C}}, \quad a_{4}=\frac{6 \beta \tau_{r t}}{\bar{C}}, \quad a_{5}=\frac{6 \beta \tau_{s t}}{\bar{C}}$

where $c=\alpha /\left(2 \sigma_{0}\right), \alpha$ and $\beta$ are material constants previously defined, and

$$
\begin{aligned}
\bar{C}= & 2\left[\left(c^{2}+\beta\right) \sigma_{r}^{2}+\left(c^{2}+\beta\right) \sigma_{s}^{2}+\left(2 c^{2}-\beta\right) \sigma_{r} \sigma_{s}\right. \\
& \left.+3 \beta\left(\tau_{r s}^{2}+\tau_{r t}^{2}+\tau_{s t}^{2}\right)\right]^{1 / 2} .
\end{aligned}
$$

The Hardening Rule The hardening rule defines the motion of the subsequent yield surface (the loading surface) during plastic deformation. It determines the relation between the loading surface (or effective stress) and the accumulated plastic strain (or effective plastic strain). The concept of effective stress and effective plastic strain makes it possible to extrapolate from a simple uniaxial test to the multiaxial condition. In the present work the relation between effective stress and effective plastic strain is extrapolated from the uniaxial stress-strain relationship using the conventional "Madrid Parabola".

$\sigma=E_{0} \varepsilon-\frac{1}{2} \frac{E_{0}}{\varepsilon_{0}} \varepsilon^{2}, \quad 0.3 f_{c}^{\prime}<\sigma<f_{c}^{\prime}$ 
Fig. 10 One-dimension representation of the concrete constitutive model

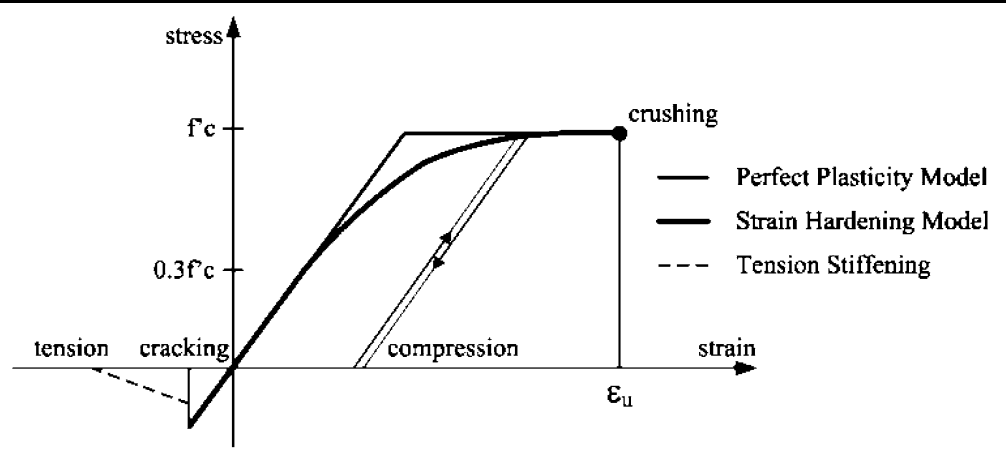

where $E_{0}$ is an initial elasticity modulus, $\varepsilon$ is the total strain, $\varepsilon_{0}$ is the total strain at peak stress $f_{c}^{\prime}$.

Substituting the elastic strain component $\varepsilon_{e}=\sigma / E_{0}$ into (136) obtains,

$\sigma=-E_{0} \bar{\varepsilon}_{p}+\sqrt{2 E_{0}^{2} \varepsilon_{0} \bar{\varepsilon}_{p}}, \quad 0.3 f_{c}^{\prime}<\sigma<f_{c}^{\prime}$

where $\bar{\varepsilon}_{p}$ is the effective plastic strain component, and $\varepsilon_{0}$ can be taken as equal to $2 f_{c}^{\prime} / E_{0}$ for normal concrete. Using the current effective plastic strain in (137) gives the effective stress level $\sigma=\sigma_{0}$, defining the current loading surface position.

The Crushing Condition The crushing of concrete is a strain-controlled phenomenon. However, there is a lack in the availability of experimental data on concrete ultimatedeformation capacity under multiaxial stress states. This has resulted in the development of an appropriate strain criterion by simply converting the yield criterion described in terms of stresses directly into strains. Thus

$\beta\left(3 J_{2}^{\prime}\right)+\alpha I_{1}^{\prime}=\varepsilon_{u}^{2}$.

$I_{1}^{\prime}$ and $J_{2}^{\prime}$ are strain invariants and $\varepsilon_{u}$ is an ultimate total strain extrapolated from uniaxial test results. The previous values of material parameter $\alpha$ and $\beta$ obtained from Kupfer's results can be adopted.

Stress-Strain Relation Based on classical theory of plasticity, the elasto-plastic stress-strain relation can be written as follow.

$d \sigma=\left(\left[C^{e}\right]-\frac{\left[C^{e}\right] a a^{\mathrm{T}}\left[C^{e}\right]}{\left(A+a^{\mathrm{T}}\left[C^{e}\right] a\right)}\right) d \varepsilon=C^{c} d \varepsilon$

where $C^{e}$ is an elastic constitutive matrix defined by:

$C^{e}=\left[\begin{array}{ccccc}\frac{E_{0}}{\left(1-v_{0}^{2}\right)} & \frac{v_{0} E_{0}}{\left(1-v_{0}^{2}\right)} & 0 & 0 & 0 \\ \frac{v_{0} E_{0}}{\left(1-v_{0}^{2}\right)} & \frac{E_{0}}{\left(1-v_{0}^{2}\right)} & 0 & 0 & 0 \\ 0 & 0 & G_{0} & 0 & 0 \\ 0 & 0 & 0 & \frac{5}{6} G_{0} & 0 \\ 0 & 0 & 0 & 0 & \frac{5}{6} G_{0}\end{array}\right]$.
$E_{0}, G_{0}$ and $v_{0}$ are the initial Young's modulus, shear modulus and Poison's ratio of concrete, respectively. The strain hardening parameter $(A)$ can be determined by differentiating (137) with respect to $\bar{\varepsilon}_{p}$.

$A=-E_{0}+\frac{E_{0}^{2} \varepsilon_{0}}{\sqrt{2 E_{0}^{2} \varepsilon_{0} \bar{\varepsilon}_{p}}}, \quad \bar{\varepsilon}_{p}>0$.

\subsubsection{Elasto-Plastic Fracture (EPF) Model in Compressive Behavior of Concrete}

In the elasto-plastic fracture model (EPF), the sources of nonlinearity can generally be classified into continuum fracture and plasticity. Continuum fracture is defined as the elasticity damage caused by dispersed defects with no clear localization. This principally results in a reduced absorbing capacity of the elastic strain energy as damages accumulate. Plasticity is defined as the unrecoverable deformation of concrete after all loads are removed. In general, continuum fracture affects ultimate strength while plasticity influences deformation of concrete. A schematic outline of an elasto-plasticity and fracturing system is illustrated in Fig. 11. Concrete is idealized as a set of infinitesimal elastoplastic components. Elasticity is modeled by parallel springs while plasticity is modeled by serially connected slider. If no springs are damaged during loading, it would have perfect elasto-plasticity, i.e. all spring would be effective in resisting stresses. However, fracture damage occurs under loading due to micro cracks and other defects. Thus, at any loading state, some springs may be broken and lose their load resisting capability. In Fig. 11, the parameter $\bar{K}$ is defined as fracture parameter representing the ratio of undamaged springs. The total stress in concrete is obtained as the summation of the internal stress carried by undamaged elements. As a result, effective stiffness is reduced by factor $\bar{K}$ as shown in Fig. 11.

The main task in describing the nonlinear behavior of concrete is the identification of continuum fracture from the overall nonlinearity. Continuum fracture would be the reduction of elastic energy absorption capability in terms of energy dissipation due to nonlinear irreversible processes. 


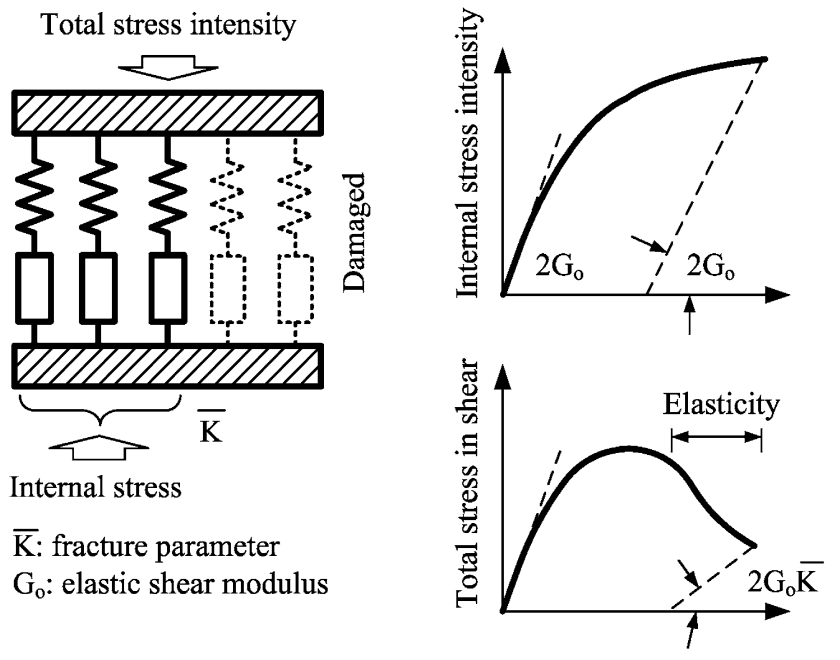

Fig. 11 Schematic outline of elasto-plastic fracture model

First, volumetric and deviatoric components of damaged elasticity will be discussed.

Volumetric Elasticity and Fracture The stiffness of the extracted elastic strain versus stress relation varies with loading paths. However, if the purely volumetric component of elasticity is extracted from the total response, it can be found that the volumetric fracturing is essentially non-existent. Maekawa et al. [93] showed that no continuum fracture is present in the volumetric elastic mode for any confinement level $\left(I_{1}\right)$ below $0.5 f_{c}^{\prime}$. This means that the entire volume of concrete is effective in absorbing and releasing the elastic strain energy under the hydrostatic component. Since concrete in practice does not have such high confinement and the stress deviator is more dominant, it acceptable to assume that there is no fracture in the volumetric mode for the continuum fracture model. Then we have the volumetric elastic constitutive law as

$I_{1}=3 K_{0} I_{1 e}$

where $I_{1}$ and $I_{1 e}$ are the first stress and elastic strain invariant express by

$I_{1}=\frac{1}{3} \sigma_{i i}, \quad I_{1 e}=\frac{1}{3} \varepsilon_{e_{i i}}$

where

$\varepsilon_{e_{i j}}=\varepsilon_{i j}-\varepsilon_{p_{i j}}$.

The strain parameters $\varepsilon_{i j}, \varepsilon_{e_{i j}}, \varepsilon_{p_{i j}}$ are the total, elastic and plastic strains, respectively. The parameter $K_{0}$ is the volumetric elastic constant equal to,

$K_{0}=\frac{E_{0}}{3\left(1-2 v_{0}\right)}$.
Elastic Deviators and Fracture

a) Deteriorated shear elasticity

Maekawa et al. [93] studied the relation of the stress and elastic strain deviator invariants in the extracted elastic mode for loading paths with different levels of confinement. When unloaded, the stress and elastic strains have to return to the origin. For different confinement levels, the unloading stiffness decreases with the progress in loading. This indicates the degradation of elasticity in the deviatoric mode. It is also shown that greater stress deviator invariant can be sustained by the damaged concrete with higher lateral confinement.

Unlike the fracture in the volumetric elastic mode, the confinement has a significant influence on the deviatoric (shear) elastic mode. Then, the fracture parameter $\bar{K}$ can be introduced into the relation between elastic stress and strain deviator invariant:

$J_{2}=2 G_{0} \bar{K} J_{2 e}$.

$J_{2}$ and $J_{2 e}$ are the second stress and elastic strain deviatoric invariant express by

$J_{2}=\sqrt{\frac{1}{2} S_{i j} S_{i j}}$,

$J_{2 e}=\sqrt{\frac{1}{2} e_{e_{i j}} e_{i j}}$,

where,

$S_{i j}=\sigma_{i j}-\delta_{i j} I_{1} \quad$ and $\quad e_{e_{i j}}=\varepsilon_{e_{i j}}-\delta_{i j} I_{1 e}$.

The value $\bar{K}$ indicates the ratio of the effective volume of concrete, which can absorb and release the shear elastic strain energy. It is positive-definite and less than unity.

b) Fracture parameter

The relation between the progress of continuum fracture (denoted by fracture parameter $\bar{K}$ ) as a function of the elastic deviator invariant is given in [93]. Higher confinement is shown to restrain the progressive fracturing to a greater degree and results in an increased deviator invariant that concrete can sustain. While the internal shear intensity advances the progress of fracture, the confinement restrains it. Thus there will be potential on the $I_{1 e}-J_{2 e}$ plane that will give the same value of the fracture parameter $\bar{K}$. Maekawa et al. [93] proposed a formulation for the fracture potential function $\bar{F}$ as a function of elastic strain invariants $I_{1 e}, J_{2 e}$.

$\bar{F}=\frac{1}{5} \cdot \frac{\sqrt{2} J_{2 e}}{0.23 \varepsilon_{0}+\sqrt{3}\left|I_{1 e}\right|}\left[6+\frac{3 \sqrt{3}}{2}\left(\frac{J_{3 e}}{J_{2 e}}\right)^{3}\right]$.

$\bar{F}$ is considered as the three dimensional equivalent fracture evolution parameter and a unique relationship between $\bar{K}$ and $\bar{F}$ is obtained [93].

$\bar{K}=\exp \left[-\frac{\bar{F}}{3.25}\left\{1-\exp \left(-\frac{\bar{F}}{0.80}\right)\right\}\right]$. 
When the fracture parameter $\bar{K}$ falls below 0.25 , compressive strain localization is observed in the concrete. Here, the strain field is no longer uniform and stress-strain relation becomes dependent on size of the control volume. Thus, this value is tentatively defined as the applicability limit of the model for continuum.

Plasticity in Shear Plasticity in shear means distorted residual deformation. Plastic flow is an irreversible pathdependent process. This means the shear plastic work adsorbed by the undamaged component varies with loading paths. Thus, the indicator of plasticity has to be pathdependent. First, we evaluate the specific shear plastic work per unit volume of active undamaged components:

$d W_{p s}=2 G_{0} e_{e_{i j}} d e_{p_{i j}}$

where

$d e_{p_{i j}}=d \varepsilon_{p_{i j}}-\delta_{i j}\left(d \varepsilon_{p_{k k}} / 3\right)$.

The relation between plastic work and plastic deviator invariant can be written as

$d J_{2 p}=\frac{d W_{p s}}{2\left(2 G_{0} J_{2 e}\right)}=\frac{e_{e_{i j}} d e_{p_{i j}}}{2 J_{2 e}}$

where $e_{e_{i j}}$ and $e_{p_{i j}}$ are the elastic and plastic strain deviator tensor, respectively.

In order to formulate the plastic constitutive law, it is assumed that the internal stress intensity, which is proportional to elastic strain, would govern the evolution of plasticity. Maekawa et al. [93] proposed the empirical polynomial equation that related the $J_{2 p}$ and $J_{2 e}$.

$J_{2 p}=H\left(J_{2 e}\right)=\frac{9}{10} \varepsilon_{0}\left(\frac{J_{2 e}}{\varepsilon_{0}}\right)^{3}$.

Equation (155) is applicable to normal aggregate concrete with strengths from 15 to $50 \mathrm{MPa}$.

Volumetric Plasticity The volumetric plasticity $I_{1 p}$ is closely related with $J_{2 p}$ and is also affected by the degree of confinement. The relation between $I_{1 p}$ and $J_{2 p}$ obtained from experimental work is provided in [93]. The change in volumetric plasticity associated with shear plasticity is known as dilatancy. The dilatancy derivative may be defined as

$\bar{D}=\frac{\partial I_{1 p}}{\partial J_{2 p}}$.

Experimentally, Maekawa et al. [93] proposed an equation of $D$ as

$\bar{D}=P(X) \tilde{D}$ where

$X=\frac{\sqrt{3} I_{1 e}}{J_{2 e}}$,

$P(X)= \begin{cases}-0.5 \sin \left(X \frac{\pi}{2}\right)+0.5 & (-1<X<1), \\ 1 & (X \leq-1), \\ 0 & (X \geq 1),\end{cases}$

$\tilde{D}= \begin{cases}\tilde{D}_{0}, & \bar{K} \geq 0.5, \\ \tilde{D}_{0}\left(2 \bar{K}^{2}\right)+\tilde{D}_{1}\left(1-4 \bar{K}^{2}\right), & \bar{K}<0.5,\end{cases}$

$\tilde{D}_{0}=-\frac{1-2 v_{0}}{\sqrt{3}\left(1+v_{0}\right)}$,

$\tilde{D}_{1}=\frac{\sqrt{2} I_{1 e}+0.38 \varepsilon_{0}}{0.28 \varepsilon_{0}}$.

Flow Rule for Elasto-Plastic Fracture Model Previous sections only established the scalar relationship between invariants. To finalize the tensorial expression of plasticity, the flow rule, which specifies the direction of plasticity, is needed. Maekawa et al. [93] assumed non-associate plasticity, where the direction of the plastic strains coincides with the elastic strains and can be written as follows:

$d e_{p_{i j}}=e_{e_{i j}} d g$

where $d g$ is the plastic strain multiplier. From (154) and (163) we can write,

$d e_{p_{i j}}=\frac{e_{e_{i j}}}{J_{2 e}} d J_{2 p}$.

The incremental plastic strain can be written in the term of the deviatoric and volumetric.

$d \varepsilon_{p_{i j}}=d e_{p_{i j}}+\delta_{i j} d I_{1 p}$.

Using (155), (156) and (164), (165) can be written as follows:

$$
\begin{aligned}
d \varepsilon_{p_{i j}} & =e_{e_{i j}} \frac{d J_{2 p}}{J_{2 e}}+\delta_{i j} \bar{D} d J_{2 p} \\
& =\left(\frac{e_{e_{i j}}}{J_{2 e}}+\delta_{i j} \bar{D}\right) \frac{d H}{d J_{2 e}} U_{p} d J_{2 e}
\end{aligned}
$$

where, $U_{p}=1$ when $J_{2 e}>0$ and $J_{2 e}=J_{2 e \max }, U_{p}=0$ otherwise.

The differentiation of $J_{2 e}$ can be expressed in terms of the elastic strain increment as follows:

$$
\begin{aligned}
d J_{2 e} & =\frac{e_{e_{k l}} d e_{e_{k l}}}{2 J_{2 e}}=\frac{e_{e_{k l}}}{2 J_{2 e}} d\left(\varepsilon_{e_{k l}}-\frac{1}{3} \delta_{k l} \varepsilon_{e_{m m}}\right) \\
& =\frac{e_{e_{k l}} d \varepsilon_{e_{k l}}}{2 J_{2 e}} .
\end{aligned}
$$


Substitute (167) into (166), finally, we obtain the plastic constitutive equation.

$d \varepsilon_{p_{i j}}=L_{i j k l} d \varepsilon_{e_{k l}}$

where,

$L_{i j k l}=\left(\frac{e_{e_{i j}}}{J_{2 e}}+\delta_{i j} \bar{D}\right) \frac{d H}{d J_{2 e}} U_{p} \frac{e_{e_{k l}}}{2 J_{2 e}}$.

Stress-Strain Relationship for Concrete in Compression by EPF Model The generalized expression of the fractured elasticity can be written as follows:

$S_{i j}=2 G_{0} \bar{K}_{i j} e_{e_{i j}}$.

Equation (170) is the general form, which accounts for the anisotropic state of fracturing damage. Maekawa et al. [93] experimentally demonstrated the isotropy of concrete shear elasticity under biaxial compression-tension stress. Since three-dimensional compressive stresses should make the internal damage more isotropic, the approximation of fracture parameter can be defined as

$\bar{K}_{i j}=\bar{K}$.

By combining the volumetric and deviatoric fracture laws of concrete, the total tensorial expression of the fractured elasticity yields

$\sigma_{i j}=\delta_{i j} I_{1}+S_{i j}=3 K_{0} I_{1 e} \delta_{i j}+2 G_{0} \bar{K} e_{e_{i j}}$.

Taking the derivative with respect to $d \varepsilon_{e_{k l}}$

$$
\begin{aligned}
d \sigma_{i j}= & M_{i j k l} d \varepsilon_{e_{k l}}, \\
M_{i j k l}= & 2 G_{0} \bar{K} \delta_{i k} \delta_{j l}+\left[\delta_{i j} 3 K_{0} \frac{d \varepsilon_{e_{l l}}}{3}\right. \\
& \left.-2 G_{0} \bar{K} \delta_{i j} \frac{d \varepsilon_{e_{l l}}}{3}\right]+2 G_{0} e_{e_{i j}} d \bar{K} .
\end{aligned}
$$

The forth order tensor $M_{i j k l}$ can be expanded as follows:

$$
\begin{aligned}
M_{i j k l}= & \text { term }_{1}+\text { term }_{2}+\text { term }_{3}, \\
\text { term }_{1}= & 2 G_{0} K \delta_{i k} \delta_{j l}, \\
\text { term }_{2}= & \frac{1}{3}\left[\left(3 K_{0}-2 G_{0} K\right) \delta_{i j}\right. \\
& +2 G_{0} e_{e_{i j}} U_{f}\left(\frac{\partial K}{\partial F}\right) \\
& \left.\times\left(\frac{\partial F}{\partial I_{1 e}}-\frac{2}{3}\left(\frac{J_{2 e}}{J_{3 e}}\right)^{2}\left(\frac{\partial F}{\partial J_{3 e}}\right)\right)\right] \delta_{k l}, \\
\text { term }_{3}= & 2 G_{0} e_{e_{i j}} U_{f}\left(\frac{\partial K}{\partial F}\right)\left[\left(\frac{\partial F}{\partial J_{2 e}}\right) \frac{e_{e_{k l}}}{2 J_{2 e}}\right. \\
& \left.+\left(\frac{\partial F}{\partial J_{3 e}}\right) \frac{e_{e_{k m}} e_{e_{l m}}}{3 J_{3 e}^{2}}\right]
\end{aligned}
$$

where, $U_{f}=1$ when $F=F_{\max }$ and $d F>0, U_{f}=0$ otherwise.

Using an elasto-plasticity concept, the strain component can be separated into elastic and plastic parts as given below.

$d \varepsilon_{i j}=d \varepsilon_{e_{i j}}+d \varepsilon_{p_{i j}}$.

Substitute (168) and (176) into (173), the matrix expression of an incremental stress-strain relation can be written as follows:

$d\{\sigma\}=M \cdot[\hat{I}+L]^{-1} d\{\varepsilon\}=C^{c} d\{\varepsilon\}$.

Equation (177) is the constitutive equation for concrete which included both fracturing and plasticity (denote that $\hat{I}$ is a unit identity matrix).

\subsubsection{Tensile Behavior of Concrete}

The response of concrete under tensile stresses is assumed to be linear elastic until the fracture surface is reached. The tensile type of fracture, or cracking, is governed by a maximum tensile stress criterion (tension cut-off). Cracks are assumed to form in planes perpendicular to the direction of maximum principal tensile stress as soon as this stress reaches the specified concrete tensile strength $f_{t}^{\prime}$. In order to avoid further complexities, cracks are assumed to form only in planes perpendicular to the structural plane ( $r, s$ plane). After cracking has occurred, the elasticity modulus and Poisson's ratio are reduced to zero in the direction perpendicular to the cracked plane, and a reduced shear modulus is employed. Taking 1 and 2 as the two principal directions in the plane of the structure, the stress-strain relationship for concrete cracking in the 1 direction, is as follow;

$$
\left\{\begin{array}{c}
\sigma_{1} \\
\sigma_{2} \\
\tau_{12} \\
\tau_{13} \\
\tau_{23}
\end{array}\right\}=\left[\begin{array}{ccccc}
0 & 0 & 0 & 0 & 0 \\
0 & E_{0} & 0 & 0 & 0 \\
0 & 0 & G_{12}^{c} & 0 & 0 \\
0 & 0 & 0 & G_{13}^{c} & 0 \\
0 & 0 & 0 & 0 & \frac{5}{6} G_{0}
\end{array}\right]\left\{\begin{array}{c}
\varepsilon_{1} \\
\varepsilon_{2} \\
\gamma_{12} \\
\gamma_{13} \\
\gamma_{23}
\end{array}\right\} .
$$

Experimental results indicate that a considerable amount of shear stress can be transferred across the rough surfaces of cracked concrete. Also, the dowel action of the steel bar contributes to the shear stiffness across cracks. These tests have shown that the primary variable in the shear transfer mechanism is the crack width, although aggregate size, reinforcement ratio and bar size also have influence. A common procedure to account for aggregate interlock and dowel action in a smeared cracking model is to attribute an appropriate value to cracked shear modulus $G^{c}$. The cracked shear modulus is assumed to be a function of the current tensile 
strain [61]. For concrete cracked in the 1 direction

$G_{12}^{c}=G_{13}^{c}=0.25 G_{0}\left(\frac{1-\varepsilon_{1}}{0.004}\right)$,

$G_{12}^{c}=G_{13}^{c}=0$ if $\varepsilon_{1}>0.004$,

$G_{23}^{c}=\frac{5}{6} G_{0}$

and for concrete cracked in both directions,

$\left\{\begin{array}{c}\sigma_{1} \\ \sigma_{2} \\ \tau_{12} \\ \tau_{13} \\ \tau_{23}\end{array}\right\}=\left[\begin{array}{ccccc}0 & 0 & 0 & 0 & 0 \\ 0 & 0 & 0 & 0 & 0 \\ 0 & 0 & G_{12}^{c} / 2 & 0 & 0 \\ 0 & 0 & 0 & G_{13}^{c} & 0 \\ 0 & 0 & 0 & 0 & G_{23}^{c}\end{array}\right]\left\{\begin{array}{c}\varepsilon_{1} \\ \varepsilon_{2} \\ \gamma_{12} \\ \gamma_{13} \\ \gamma_{23}\end{array}\right\}$

where,

$G_{13}^{c}=0.25 G_{0}\left(\frac{1-\varepsilon_{1}}{0.004}\right), \quad G_{13}^{c}=0 \quad$ if $\varepsilon_{1}>0.004$,

$G_{23}^{c}=0.25 G_{0}\left(\frac{1-\varepsilon_{2}}{0.004}\right), \quad G_{22}^{c}=0 \quad$ if $\varepsilon_{2}>0.004$,

$G_{12}^{c}=0.5 G_{13}^{c} \quad$ or $\quad G_{12}^{c}=0.5 G_{23}^{c} \quad$ if $G_{23}^{c}<G_{13}^{c}$.

If the crack closes the uncracked shear modulus $G_{0}$ is again assumed in the corresponding direction.

\subsubsection{Tension Stiffening}

Due to bond effects, cracked concrete carries between cracks a certain amount of tensile force normal to the cracked plane. The concrete adheres to the reinforcing bars and contributes to the overall stiffness of the structure. Several approaches based on experimental results have been employed to simulate this tension stiffening behavior. A gradual release of the concrete stress component normal to the cracked plane is adopted in this work. The process of loading and unloading of cracked concrete is also illustrated in Fig. 12. Unloading and reloading of cracked concrete is assumed to follow the linear behavior shown with a fictitious elasticity modulus $E_{i}$ given by

$E_{i}=\alpha_{t} f_{t}^{\prime} \frac{\left(\frac{1-\varepsilon_{i}}{\varepsilon_{m}}\right)}{\varepsilon_{i}}, \quad \varepsilon_{t} \leq \varepsilon_{i} \leq \varepsilon_{m}$.

$\alpha_{t}$ and $\varepsilon_{m}$ are tension stiffening parameters (Fig. 12) and $\varepsilon_{i}$ is the maximum value reached by the tensile strain at the point considered. If the crack closes, i.e. if the strain component normal to the crack plane becomes negative, the concrete acquires the uncracked behavior in the corresponding direction, but the crack direction and the maximum tensile strain continue to be stored. The value $\varepsilon_{i}$ can be readily modified to simulate bond deterioration during reloading. The

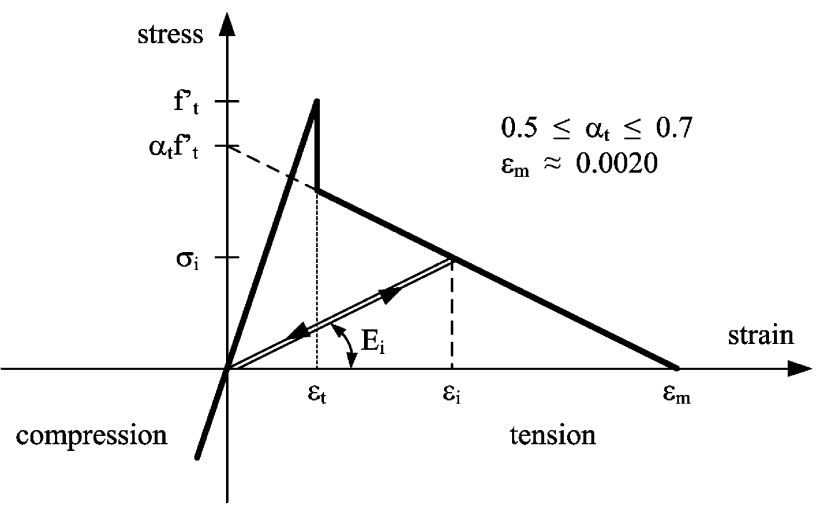

Fig. 12 Loading and unloading behavior of cracked concrete (tension stiffening diagram)

normal stress $\sigma_{1}$ (and or $\sigma_{2}$ ) is obtained by the following expression.

$\sigma_{1}=\alpha_{t} f_{t}^{\prime}\left(\frac{1-\varepsilon_{1}}{\varepsilon_{m}}\right), \quad \varepsilon_{t} \leq \varepsilon_{1} \leq \varepsilon_{m}$

or by

$\sigma_{1}=\frac{\sigma_{i} \varepsilon_{1}}{\varepsilon_{i}}, \quad \varepsilon_{1} \leq \varepsilon_{i}$

where $\varepsilon_{1}$ is the current tensile strain in material direction 1 .

The value of $f_{t}^{\prime}$ should be taken as the modulus of rupture of the concrete. This modulus can be related to the uniaxial compression strength by

$f_{t}^{\prime}=f_{r}=0.62 \sqrt{f_{c}^{\prime}}$

where $f_{t}^{\prime}, f_{r}$ and $f_{c}^{\prime}$ are expressed in MPa. However experimental tests have shown large variations in the above coefficient. The tension stiffening constant $\alpha$ in Fig. 12 is taken equal to $0.5,0.6$ or 0.7 depending on the relative percentage of steel in the section. However the change in structural behavior with this variation is generally small and a constant value of $\alpha=0.6$ could be used. For the tension stiffening constant, $\varepsilon_{m}$, a fixed value of 0.002 is employed.

\subsubsection{Tension and Compression Behavior of Reinforcing Steel}

Reinforcing steel is modeled as a bilinear material and stress reversal with Bauschinger effect is included, as shown in Fig. 13. Four parameters are necessary as input data to define the steel model: the initial Young's modulus $E_{s 1}$, the strain hardening modulus $E_{s 2}$, the yield stress $f_{y}$ and the ultimate strain $\varepsilon_{s u}$. The steel layers are considered as a onedimensional material in the reinforcement direction. 
4.4.6 Constitutive Relation of Reinforced Concrete Material

An incremental constitutive law for each layer of the shell is expressed as follows:

$$
\begin{gathered}
\left\{\begin{array}{c}
\Delta \sigma_{r} \\
\Delta \sigma_{s} \\
\Delta \tau_{r s} \\
\Delta \tau_{r t} \\
\Delta \tau_{s r}
\end{array}\right\}=\left[\begin{array}{ccccc}
C_{11} & C_{12} & C_{13} & 0 & 0 \\
C_{21} & C_{22} & C_{23} & 0 & 0 \\
C_{31} & C_{32} & C_{33} & 0 & 0 \\
0 & 0 & 0 & C_{44} & 0 \\
0 & 0 & 0 & 0 & C_{55}
\end{array}\right]\left\{\begin{array}{c}
\Delta \hat{e}_{r} \\
\Delta \hat{e}_{s} \\
\Delta \hat{e}_{r s} \\
\Delta \hat{\gamma}_{r t} \\
\Delta \hat{\gamma}_{s t}
\end{array}\right\} \\
=\left[\begin{array}{cc}
\mathbf{C}_{m b}^{c} & 0 \\
0 & \mathbf{C}_{q}^{c}
\end{array}\right]\left\{\begin{array}{c}
\Delta \hat{\mathbf{e}}_{m b} \\
\Delta \hat{\mathbf{e}}_{q}
\end{array}\right\}=\mathbf{C}^{c} \Delta \hat{\mathbf{e}}_{l} .
\end{gathered}
$$

The constitutive matrix $\mathbf{C}^{c}$ is already given in (139) and (177) for plasticity-based and EPF model, respectively. The rigidity matrix in (86) can be determined by integrating the

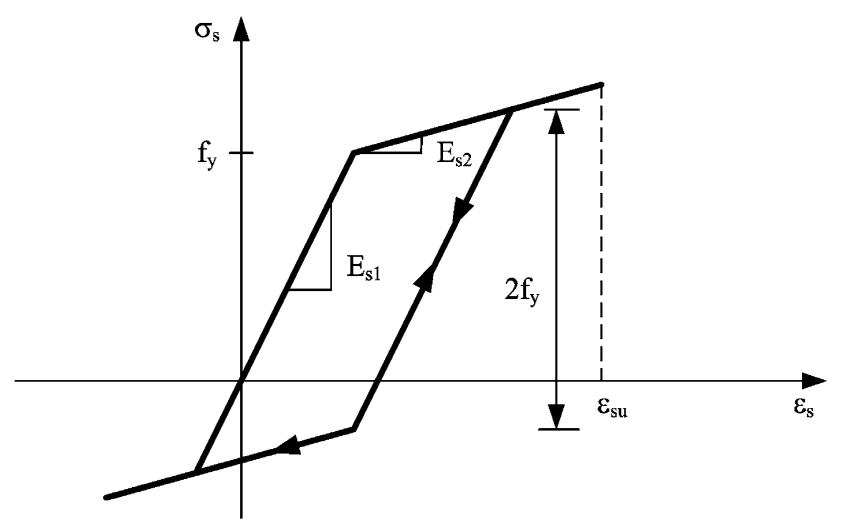

Fig. 13 Stress-strain relationship of the reinforcing steel

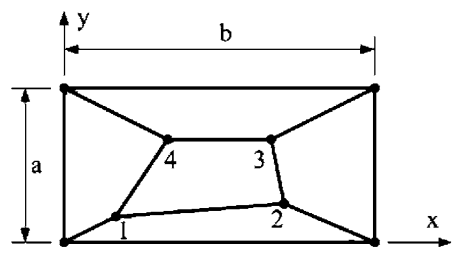

Fig. 14 Shell element patch test rigidity from each layer as follows:

$$
\mathbf{D}=\left[\begin{array}{ccc}
\int_{-2 / h}^{2 / h} \mathbf{C}_{m b}^{c} d t & \int_{-2 / h}^{2 / h} \mathbf{t C}_{m b}^{c} d t & \mathbf{0} \\
\int_{-2 / h}^{2 / h} \mathbf{t C}_{m b}^{c} d t & \int_{-2 / h}^{2 / h} \mathbf{t}^{2} \mathbf{C}_{m b}^{c} d t & \mathbf{0} \\
\mathbf{0} & \mathbf{0} & \int_{-2 / h}^{2 / h} \mathbf{C}_{q}^{c} d t
\end{array}\right]
$$

In order to incorporate the nonlinear material behavior, integration of (187) must evidently be carried out numerically and the mid-ordinate rule is used for concrete and steel layers.

\section{Numerical Results}

Results to the nonlinear analysis of plate and shell problems are presented to validate the numerical performance of the formulation. The present nonlinear formulation of the 4-node quasi-conforming shell element, XSHELL41, is implemented into the general purpose Nonlinear Dynamic Finite Element Package, XFINAS [178], developed in AIT and Konkuk University. XFINAS is an extended version of the nonlinear finite element package FINAS, developed in Imperial College, London. It runs on a personal computer with the personal pre and postprocessor software GiD [51] developed by CIMNE in Spain. The PC used for the following examples runs on Windows XP operating system.

\subsection{Patch Test}

The patch test used for verifying the present shell elements is found in MacNeal and Harder (1985) [92]. The test involves membrane and bending tests. The boundary conditions are given in Table 2 and the patch mesh is shown in Fig. 15. $a=0.12, b=0.24, t=0.001 . E=1 \mathrm{e} 6, v=0.25$.

\subsection{Linear Isotropic Static Problems}

A list of shell elements used for comparison with the proposed elements is as follows:

QUAD4-Isoparametric formulation with selective reduced integration, MacNeal and Harder [92].
Table 2 Coordinates and boundary conditions

\begin{tabular}{lllll}
\hline & $x$ & $y$ & (a) Membrane Patch Test & \\
1 & 0.04 & 0.02 & Boundary Conditions: & $u=10^{-3}(x+y / 2)$ \\
2 & 0.18 & 0.03 & & $v=10^{-3}(y+x / 2)$ \\
3 & 0.16 & 0.08 & (b) Bending Patch Test & \\
4 & 0.08 & 0.08 & Boundary Conditions: & $w=10^{-3}\left(x^{2}+x y+y^{2}\right) / 2$ \\
& & & & $\theta_{x}=\partial w / \partial y=10^{-3}(x / 2+y)$ \\
& & & $\theta_{y}=-\partial w / \partial x=10^{-3}(-x-y / 2)$ \\
\hline
\end{tabular}


S4R5-Bilinear shell with mixed formulations used for the membrane and bending stresses and full $2 \times 2$ quadrature in ABACUS by Hibbitt, Karlsson \& Sorensen, Inc [59].

Simo et al. (1989) [138, 144] —Bilinear shell with mixed formulation used for the membrane and bending stresses and full $2 \times 2$ quadrature.

MITC4 - 4-node fully integrated shell element derived by Dvorkin and Bathe [42]. The shear strains are determined using the assumed strain interpolation.

QPH-Quadrilateral shell element with physical hourglass control by Belytschko and Leviathan $[17,18]$.

Straight Cantilever Beam The following are simple linear problems. A cantilever beam is subjected to four different types of loading, tensile, in-plane and out-of-plane, and torsion loadings. The cantilever beam length is $L=6.0$, the cross-section height $h=0.20$ and thickness is $t=0.10$. The beam depth is small compared to its length, so the shear contribution to the transverse deformation is small. Three mesh conditions are considered, regular, trapezoidal, and parallel-

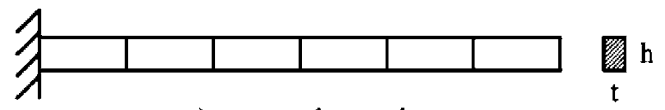

a) rectangular mesh

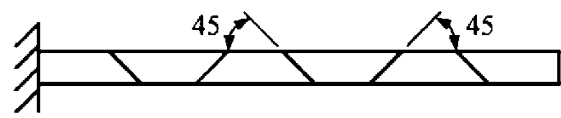

b) trapezoidal mesh

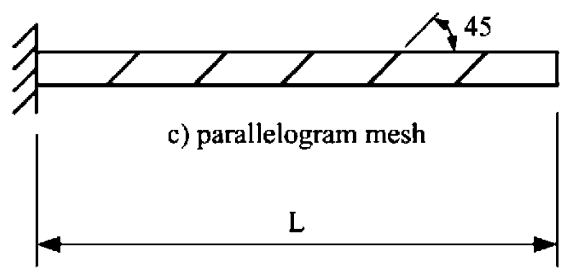

Fig. 15 Straight cantilever beam

Table 3 Maximum error in stress (\%)

\begin{tabular}{ll}
\hline & XSHELL41 \\
\hline Membrane Test & 0.038 \\
Bending Test & 0.045 \\
\hline
\end{tabular}

ogram mesh. The elastic modulus $E=1.0 \mathrm{e} 7$ and Poisson's ratio $v=0.30$. The loadings are all unit loads applied at the beam tip.

The problem is solved to demonstrate the elements capability in handling meshes with distortions and high aspect ratios. For in-plane problems, XSHELL41 and QUAD4 lock for the plane shear problem. For the out-of-plane shear problem, the results of XSHELL41 are about the same as QUAD4, only showing less sensitivity to mesh distortion. However, for the twist problem, QUAD4 provides better results.

Curved Cantilever Beam A curved cantilever beam subjected to, first, an in-plane unit point load at the free end, and second, an out-of-plane unit point load. The curved beam forms a quarter circle. It has an outer radius $R_{o}=4.32$, inner radius $R_{i}=4.12$, and thickness of $t=0.10$. The elastic modulus $E=1.0 \mathrm{e} 7$ and Poisson's ratio $v=0.25$.

Quadrilateral elements used for analyzing the curved beam would unavoidably be distorted. In addition to this, a coarse mesh of $1 \times 6$ would make a high aspect ratio for each element. Similar to the results on the previous problem, XSHELL41 provides good results for the in-plane problem but QUAD4 provides better results for out of plane shear when the mesh requires a high aspect ratio.

Twisted Cantilever Beam The following problem is to test an element's ability to model warped geometry. The beam is clamped in one end and free in the other. The free end is twisted by ninety degrees. Two loading conditions are considered, an in-plane and an out-of-plane unit load at the beam tip. The beam has length $L=12$, width $w=1.0$ and thickness $t=0.32$. The material properties are $E=29 \mathrm{e} 6$ and $v=0.22$.

For the XSHELL41, the warping correction suggested in Taylor [155] was required to provide good result, shown in Table 6.

Tapered and Swept Beam This problem demonstrates an element's ability to solve problems undergoing both shear and bending. Because of the shape of the beam, elements used in meshing are distorted. The beam dimensions are shown in Fig. 18. The modulus of elasticity $E=1.0$, Pois-
Table 4 Normalized results to the straight beam problem

\begin{tabular}{|c|c|c|c|c|c|c|c|}
\hline \multirow[t]{2}{*}{ Load } & \multirow{2}{*}{$\begin{array}{l}\text { Analytical } \\
\text { Solution }\end{array}$} & \multicolumn{3}{|c|}{ QUAD4 } & \multicolumn{3}{|c|}{ XSHELL41 } \\
\hline & & $\mathrm{a}$ & $\mathrm{b}$ & $\mathrm{c}$ & $\mathrm{a}$ & $\mathrm{b}$ & $\mathrm{c}$ \\
\hline Extension & $3.0 \times 10^{-5}$ & 0.995 & 0.996 & 0.996 & 0.995 & 0.985 & 1.010 \\
\hline In-plane Shear & 0.1081 & 0.904 & 0.071 & 0.080 & 0.904 & 0.091 & 0.115 \\
\hline Out-of-plane shear & 0.4321 & 0.986 & 0.968 & 0.977 & 0.987 & 0.987 & 0.985 \\
\hline Twist & 0.03208 & 0.941 & 0.951 & 0.945 & 0.738 & 0.761 & 0.769 \\
\hline
\end{tabular}


son's ratio $v=0.33$ and thickness $h=1.0$. The converged reference solution is 23.91. The XSHELL41 element gives

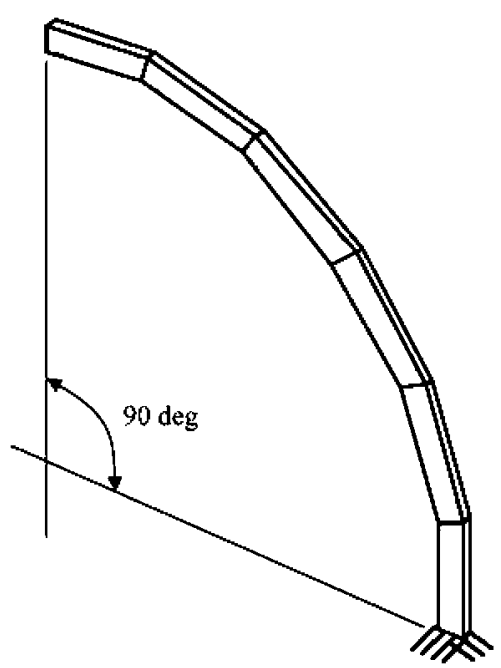

Fig. 16 Curved beam

Table 5 Normalized results of curved beam

\begin{tabular}{lll}
\hline Load (theory) & QUAD4 & XSHELL41 \\
\hline In-plane shear (0.08734) & 0.833 & 0.917 \\
Out-of-plane shear (0.5022) & 0.951 & 0.864 \\
\hline
\end{tabular}

Table 6 Normalized results of twisted beam

\begin{tabular}{llllll}
\hline \multirow{2}{*}{ Mesh Size } & \multicolumn{2}{l}{ QUAD4 } & & \multicolumn{2}{l}{ XSHELL41 } \\
\cline { 5 - 6 } & In-plane & Out-of-plane & & In-plane & Out-of-plane \\
\hline $1 \times 6$ & - & - & 0.971 & 1.013 \\
$2 \times 12$ & 0.991 & 0.985 & 0.976 & 1.060 \\
$4 \times 24$ & - & - & 0.997 & 0.999 \\
\hline
\end{tabular}

good results compared to the results of Simo et al. [138, 144].

Clamped Plate A clamped square and rectangular plate subjected to a concentrated load at the center is analyzed to convergence. The purpose is to test the elements performance in bending of a thin plate, no shear contribution, and bending with a high aspect ratio. The square plate has a side length of $a=b=2.0$ and the rectangular plate has side lengths of $a=2.0$ and $b=10.0$. For both plates, the thickness is $t=0.0001$. The material properties used for the plate are $E=1.7472 \mathrm{e} 7$ and $v=0.30$. The concentrated load at the plate center is $P=4.0 \mathrm{e}-4$. The analytical solution is from Timoshenko and Woinowsky-Krieger [157].

Only one quarter of the plate is used in the model. XSHELL41 gives very good results for both the thin square and rectangular plates and do not show any shear locking problems.

Hemispherical Shell To give good results for this problem, two properties must be demonstrated by the shell elements. An inextensional bending mode must be allowed and secondly, a rigid body motion must be well expressed. The hemisphere has a radius $r=10.0$ and thickness $t=0.04$. An 18-degree hole is located at the top of the hemisphere. Inward and outward forces in perpendicular directions are applied at the base. The material properties are $E=6.825 \mathrm{e} 7$ and $v=0.30$.

The problem is modeled using only one quarter of the hemisphere. Two reference solutions are available; the result from Simo et al. [138, 144] is 0.093 and from MacNeal and Harder [92] is 0.094 .

The mesh is increased from $2 \times 2$ elements to $16 \times 16$ elements. The QUAD4 element gives very good results for this problem, while XSHELL41 gives similar result to the

Fig. 17 Twisted cantilever beam

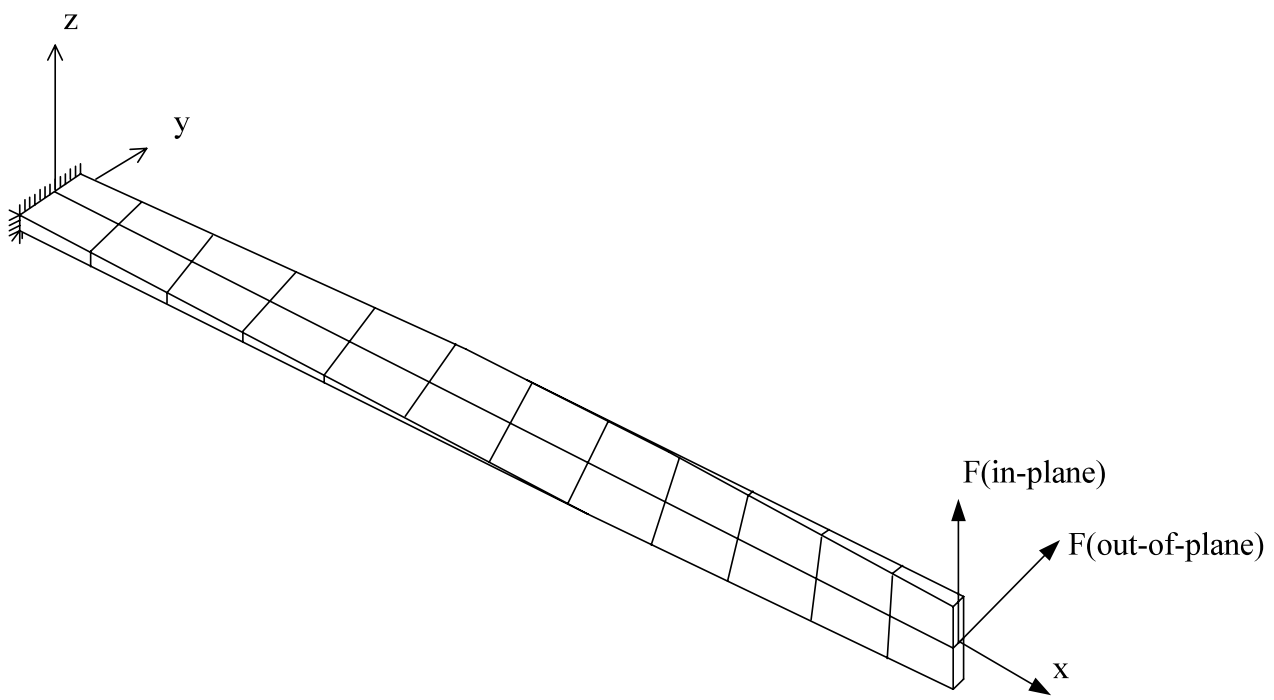




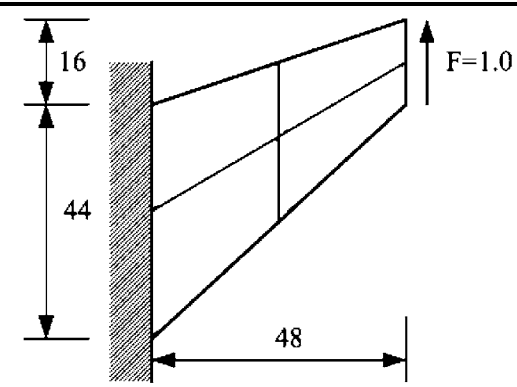

Fig. 18 Tapered and swept beam

Table 7 Cook's tapered and swept beam normalized results— 4 node

\begin{tabular}{lll}
\hline Mesh & Simo et al. (1989) [55] & XSHELL41 \\
\hline $2 \times 2$ & 0.883 & 0.816 \\
$4 \times 4$ & 0.963 & 0.937 \\
$8 \times 8$ & 0.991 & 0.983 \\
$16 \times 16$ & 0.999 & 0.997 \\
\hline
\end{tabular}

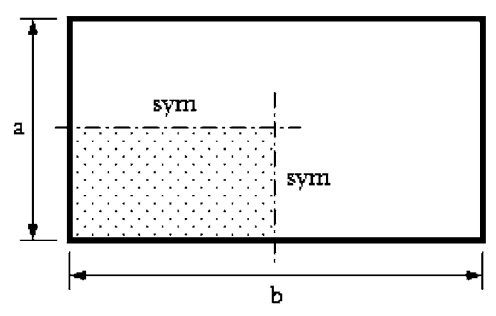

(a) mesh $\mathrm{A}$

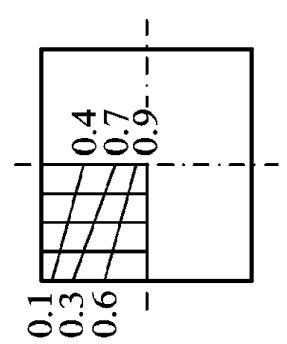

(b) distorted mesh $\mathrm{C}$
Fig. 19 Clamped square and rectangular plates

Table 8 Clamped square and rectangular plates normalized results

\begin{tabular}{llllll}
\hline Mesh & \multicolumn{2}{l}{$a / b=1(w=5.60)$} & & \multicolumn{2}{c}{$a / b=5(w=7.23)$} \\
\cline { 2 - 3 } & XSHELL41 & QUAD4 & & XSHELL41 & QUAD4 \\
\hline $2 \times 2$ & 0.960 & 0.934 & & 0.709 & 0.519 \\
$4 \times 4$ & 0.992 & 1.010 & & 0.929 & 0.863 \\
$6 \times 6$ & 0.998 & 1.012 & 0.963 & 0.940 \\
$8 \times 8$ & 1.000 & 1.010 & & 0.973 & 0.972 \\
Distorted & 0.995 & - & - & - \\
\hline
\end{tabular}

results of Simo et al. [138, 144] for meshes $8 \times 8$ and $16 \times$ 16.

Pinched Cylinder A short cylinder with rigid end diaphragms subjected to two pinching forces is analyzed. This is one of the most severe tests of an element's ability to model both inextensional bending and complex membrane states. The cylinder has a length $L=600.0$, radius $r=300.0$ and thickness $t=3.0$. The material properties are $E=3.0 \mathrm{e} 7$ and $v=0.30$. The radial displacement at the location of the point load is $1.8248 \mathrm{e}-5$.

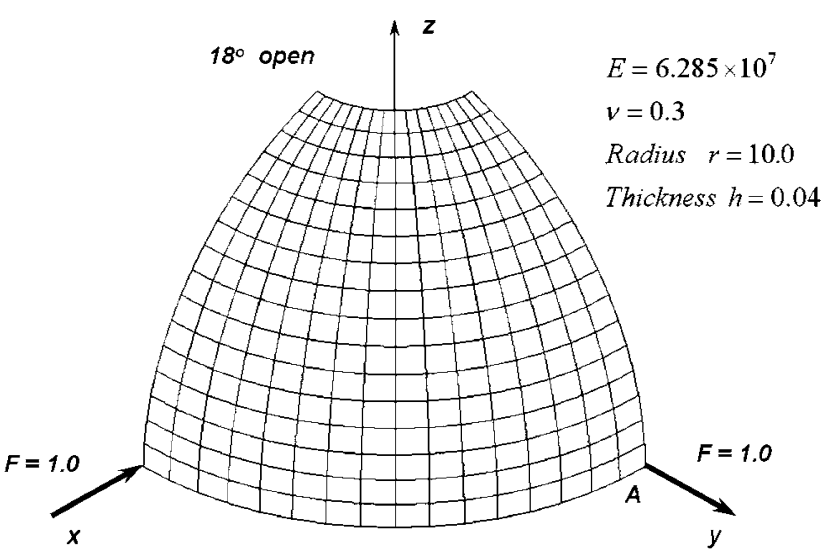

Fig. 20 Hemispherical shell with $18^{\circ}$ hole

Table 9 Hemispherical shell with $18^{\circ}$ hole normalized results (0.093)

\begin{tabular}{clll}
\hline Elements per Side & Simo et al. & QUAD4 & XSHELL41 \\
\hline 2 & 0.919 & 0.972 & 1.038 \\
4 & 1.004 & 1.024 & 1.038 \\
8 & 0.998 & 1.005 & 1.012 \\
16 & 0.999 & - & 1.001 \\
\hline
\end{tabular}

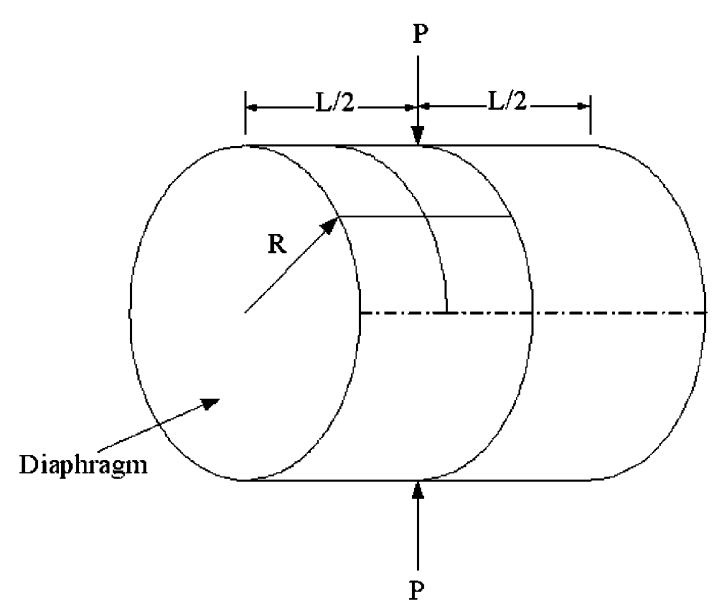

Fig. 21 Pinched cylinder

Table 10 Pinched cylinder normalized results

\begin{tabular}{llllll}
\hline Mesh & S4R5 & MITC4 & QPH & Simo et al. & XSHELL41 \\
\hline $4 \times 4$ & - & 0.37 & 0.37 & 0.399 & 0.625 \\
$6 \times 6$ & 0.6022 & - & - & - & - \\
$8 \times 8$ & - & 0.74 & 0.74 & 0.763 & 0.926 \\
$11 \times 11$ & 0.875 & - & - & - & - \\
$16 \times 16$ & - & 0.93 & 0.93 & 0.935 & 0.995 \\
$21 \times 21$ & 0.974 & - & - & - & - \\
\hline
\end{tabular}

Only one octant of the cylinder is used to model the problem. Very good results are obtained using XSHELL41 when 
Fig. 22 Scordelis-Lo roof

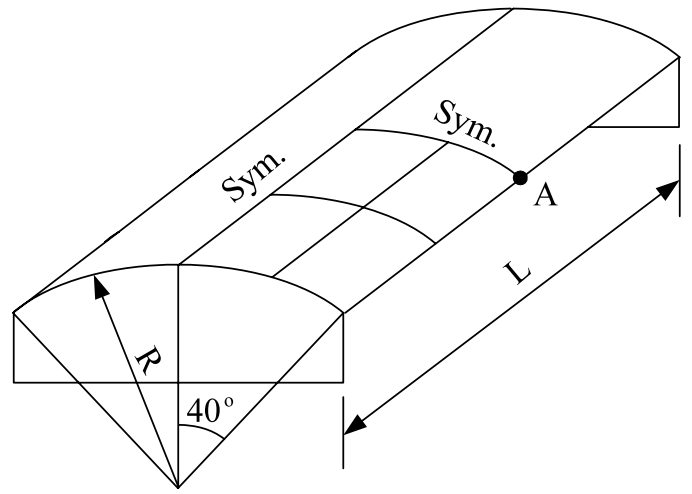

\author{
$E=4.32 \times 10^{8}$ \\ $v=0.0$ \\ $L=50$ \\ $R=25$ \\ $h=0.25$ \\ Gravity load $=90$
}

Table 11 Scordelis-Lo roof problem normalized results -4 node

\begin{tabular}{llllll}
\hline Mesh & MITC4 & QPH & Simo et al. & QUAD4 & XSHELL41 \\
\hline $4 \times 4$ & 0.94 & 0.94 & 1.083 & 1.050 & 1.035 \\
$6 \times 6$ & - & - & - & 1.018 & 1.009 \\
$8 \times 8$ & 0.97 & 0.98 & 1.015 & 1.008 & 1.002 \\
$10 \times 10$ & - & - & - & 1.004 & 0.999 \\
$16 \times 16$ & 1.00 & 1.01 & 1.000 & - & - \\
\hline
\end{tabular}

compared with the references. The ability of the present elements to analyze this problem is attributed to the cubic interpolation used in the string functions for bending whereas the elements used in the references use bilinear formulations.

Scordelis-Lo Roof The Scordelis-Lo roof is a short cylinder section supported by rigid diaphragms at the edges. It is a membrane-dominated problem. It has a length $L=50.0$, radius $r=25.0$ and thickness $t=0.25$. The material properties are $E=4.32 \mathrm{e} 8$ and $v=0.0$. The load due to gravity is 90.0 per unit area. The reference solution for the vertical displacement of point a on the free edge is 0.3024 .

Taking advantage of symmetry, only one quarter is used in the analysis of the problem. The XSHELL41 element gives very good results compared with references.

\subsection{Linear Laminate Composite Static Problems}

Simply-Supported Angle-ply Laminated Plates $(\theta,-\theta)$

Angle-ply laminated composite plate subjected to uniform lateral loading is analyzed. Different aspect ratios are used to verify the shell element for thick and thin plates. The problem geometry and material properties are as follows:

$$
\begin{aligned}
& a=b=20 \text { in. } \quad a / h=10,25,50,100 \\
& E_{1}=25.0 \mathrm{e} 6 \mathrm{psi} \quad E_{2}=1.0 \mathrm{e} 6 \mathrm{psi} \quad v=0.25 \\
& G_{12}=G_{13}=0.5 \mathrm{e} 6 \mathrm{psi} \quad G_{23}=0.2 \mathrm{e} 6 \mathrm{psi} .
\end{aligned}
$$

The results are presented in the non-dimensional form using the equation,

$$
\bar{W}=\left(\frac{h^{3} E_{2} 10^{3}}{q_{o} a^{4}}\right) W_{c}
$$

and are presented in Table 12. The results show that for angle-ply plates, the shell element is good for thin and moderately thick, but the convergence for thick plates is slow. The reference solutions of Whitney [167] are obtained by a series solution and the answers by Pucha and Reddy [111] are obtained by a finite element solution.

Very Thin Simply-Supported Angle-ply Laminated Plates $(\theta,-\theta)$ Using the same material properties as the previous problem, a very thin plate, $a / h=1000$, subjected to uniform lateral loading is analyzed and the results are shown in Table 13. The shell element gives very good results for the very thin plate case.

Clamped Angle-Ply Laminated Plates $(\theta,-\theta)$ The nondimensional center deflections of very thin plates, $a / h=$ 1000, having clamped edges are shown in Table 14. The plates are subjected to uniform lateral loading and the material properties are the same as previous problem. The shell element compares well with the reference solutions.

Clamped Cross-Ply Laminated Plate (0/90) A clamped cross-ply laminated plate subjected to uniform lateral pressure is analyzed. The aspect ratio for the plates is $a / h=$ 1000 and the material properties are the same as the previous problems. The results compare well with the analytical solution of Whitney [167].

Simply Supported Laminated Spherical Shell A laminated spherical shell having a geometry shown in Fig. 23 is analyzed for center deflection when subjected to a uniform inward pressure. Lay-ups of (0/90/0/90/0/90/0/90/0) and $(45 /-45 / 45 /-45 / 45 /-45 / 45 /-45 / 45)$ are considered with span to thickness ratios, $a / h$, of $0.1,0.01$ and 0.001 . 
Table 12 Non-dimensional center deflection of angle-ply plates of varying aspect ratio

\begin{tabular}{|c|c|c|c|c|c|c|c|}
\hline \multirow[t]{2}{*}{$a / h$} & \multirow[t]{2}{*}{$\theta$} & \multirow[t]{2}{*}{ Whitney } & \multirow[t]{2}{*}{ Putcha \& Reddy } & \multicolumn{4}{|c|}{ XSHELL41 } \\
\hline & & & & $4 \times 4$ & $8 \times 8$ & $16 \times 16$ & $32 \times 32$ \\
\hline \multirow[t]{4}{*}{10 (thick) } & $\theta=5$ & 10.0117 & 10.079 & 6.649 & 7.221 & 7.726 & 8.584 \\
\hline & $\theta=15$ & 12.2858 & 11.842 & 8.580 & 9.646 & 10.320 & 11.160 \\
\hline & $\theta=30$ & 13.2337 & 11.749 & 9.224 & 10.690 & 11.600 & 12.520 \\
\hline & $\theta=45$ & 12.7954 & 11.582 & 8.831 & 10.200 & 11.080 & 12.000 \\
\hline \multirow[t]{4}{*}{25 (thick) } & $\theta=5$ & 7.5191 & 7.5853 & 6.444 & 6.915 & 7.042 & 7.116 \\
\hline & $\theta=15$ & 10.0133 & 9.5552 & 8.320 & 9.289 & 9.545 & 9.657 \\
\hline & $\theta=30$ & 11.1228 & 9.5898 & 8.989 & 10.320 & 10.660 & 10.810 \\
\hline & $\theta=45$ & 10.6816 & 9.4733 & 8.642 & 9.910 & 10.220 & 10.360 \\
\hline \multirow[t]{4}{*}{50 (moderately thick) } & $\theta=5$ & 7.1581 & 7.2218 & 6.420 & 6.891 & 7.006 & 7.039 \\
\hline & $\theta=15$ & 9.6866 & 9.2024 & 8.288 & 9.262 & 9.504 & 9.569 \\
\hline & $\theta=30$ & 10.8215 & 9.2624 & 8.956 & 10.300 & 10.620 & 10.710 \\
\hline & $\theta=45$ & 10.3807 & 9.1736 & 8.614 & 9.894 & 10.190 & 10.260 \\
\hline \multirow[t]{4}{*}{100 (thin) } & $\theta=5$ & 7.0676 & 7.1298 & 6.414 & 6.886 & 7.000 & 7.030 \\
\hline & $\theta=15$ & 9.6049 & 9.1077 & 8.280 & 9.256 & 9.498 & 9.559 \\
\hline & $\theta=30$ & 10.746 & 9.1718 & 8.948 & 10.300 & 10.620 & 10.700 \\
\hline & $\theta=45$ & 10.3035 & 9.0973 & 8.606 & 9.891 & 10.190 & 10.260 \\
\hline
\end{tabular}

Table 13 Non-dimensional center deflection of angle-ply plates

\begin{tabular}{|c|c|c|c|c|c|}
\hline \multirow[t]{2}{*}{$\begin{array}{l}\text { Aspect ratio } \\
a / h=1000\end{array}$} & \multicolumn{2}{|c|}{$\begin{array}{l}\text { Spilker } \\
\text { \& Engelmann } \\
(1986)[145]\end{array}$} & \multicolumn{3}{|c|}{$\begin{array}{l}\text { XSHELL41 } \\
\text { Mesh(full) }\end{array}$} \\
\hline & Exact & FEM & $8 \times 8$ & $16 \times 16$ & $32 \times 32$ \\
\hline$\theta=5$ & 4.736 & 4.756 & 4.637 & 4.710 & 4.729 \\
\hline$\theta=15$ & 7.142 & 7.135 & 6.882 & 7.076 & 7.125 \\
\hline$\theta=25$ & 7.870 & 7.840 & 7.548 & 7.791 & 7.851 \\
\hline$\theta=35$ & 7.561 & 7.535 & 7.253 & 7.485 & 7.542 \\
\hline$\theta=45$ & 7.320 & 7.310 & 7.034 & 7.252 & 7.304 \\
\hline
\end{tabular}

The spherical shell has a radius $R=10.0$, width $a=1.0$ and edge height $f=0.125$. The material properties are $E_{1} / E_{2}=40, G_{12} / E_{2}=0.60, G_{23} / E_{2}=0.50$ and $v_{12}=$ 0.25 . Similar to the angle-ply cases, the present element converges slowly when used for thick shells but performs well for thin shells. The results are tabulated in Table 16.

Linear Buckling Buckling eigenvalues are determined for flat plates subject to in-plane compressive loading. Four cases are considered, i.e. lay-up of $(\theta 20)$ and $(\theta,-\theta) 5 \mathrm{~s}$ for both simply supported and clamped supports. The plate properties for the simple plate are $E_{y} / E_{x}=0.1, G_{x y} / E_{x}=$ $0.03, v=0.3$ and $a / b=1.13$, while for the clamped plate, are $E_{y} / E_{x}=0.09, G_{x y} / E_{x}=0.03, v=0.3$ and $a / b=1.0$. The results along with the analytical solution by Zhang [182] are presented in Figs. 24 and 25.

\subsection{Nonlinear Isotropic \& Elasto-Plastic Problems}

Square Clamped Plate Subjected to Uniform Pressure A very thin plate is subjected to an increasing uniform pressure. The plate is thin enough that the effect of shear is negligible. The plate length is $a=1000.0$ and thickness $t=2.0$. The modulus of elasticity is $E=2.0 \mathrm{e} 4$ and Poisson ratio $v=0.30$. The analytical solution is given by Timoshenko and Woinowsky-Krieger [157].

Sixteen XSHELL41 elements are used to model one quarter of the plate. The present four-node element matches with the analytical reference solution.

Hinged Cylindrical Shell The snap through behavior of two cylindrical shells subjected to a point load at the center is analyzed. One shell is $12.7 \mathrm{~mm}$ thick and the other is $6.35 \mathrm{~mm}$ thick. All other properties are the same. The cylinder geometric properties are length $L=508.0 \mathrm{~mm}$, radius $R=2540.0 \mathrm{~mm}$, arc half angle $\theta=0.1$ radian. The modulus of elasticity $E=3.10275 \mathrm{kN} / \mathrm{mm}^{2}$ and Poisson's ratio $v=0.30$. The cylindrical shell is simply supported along the longitudinal boundaries and unsupported along the curved edges.

Only one quarter of the problem is modelled using a mesh with $8 \times 8$ XSHELL41 elements. The thicker shell $(12.7 \mathrm{~mm})$ has a typical snap-through behaviour while the thinner shell $(6.35 \mathrm{~mm})$ behaves with a snap-back behaviour. Two mesh configurations are considered. The results show good agreement with the reference solutions by Sabir and Lock [126], and Ramm [113]. Also, present quasiconforming solution gives better comparison than the quasi- 
Table 14 Non-dimensional center deflection of angle-ply plates with clamped edges

\begin{tabular}{llllll}
\hline $\begin{array}{l}\text { Aspect ratio } \\
a / h=1000\end{array}$ & $\begin{array}{l}\text { Whitney (1970) } \\
{[168] \text { analytical }}\end{array}$ & $\begin{array}{l}\text { Spilker \& Engelmann } \\
(1986)[145]\end{array}$ & $\begin{array}{l}\text { Zaghloul \& Kennedy } \\
(1975)[180]\end{array}$ & $\begin{array}{l}\text { XSHELL41 } \\
\text { Mesh (full) }\end{array}$ \\
\hline$\theta=5$ & 0.946 & 1.070 & 1.184 & 1.031 & 1.042 \\
$\theta=15$ & 1.691 & 1.965 & - & 1.881 & 1.964 \\
$\theta=25$ & 2.355 & 2.467 & 2.890 & 2.387 & 2.541 \\
$\theta=35$ & 2.763 & 2.734 & 3.172 & 2.620 & 2.824 \\
$\theta=45$ & 2.890 & 2.850 & 3.182 & 2.689 & 2.911 \\
\hline
\end{tabular}

Table 15 Non-dimensional center deflection of clamped cross-ply laminate

\begin{tabular}{|c|c|c|c|c|}
\hline \multirow{3}{*}{$\begin{array}{l}\text { Whitney } \\
\text { (1969) [167] } \\
\text { analytical }\end{array}$} & \multirow{3}{*}{$\begin{array}{l}\text { Zaghloul } \\
\text { \& Kennedy } \\
\text { (1975) [180] }\end{array}$} & \multicolumn{3}{|c|}{ XSHELL41 (full plate mesh) } \\
\hline & & $8 \times 8$ & $16 \times 16$ & $32 \times 32$ \\
\hline & & & & \\
\hline 2.861 & 3.113 & 2.731 & 2.845 & 2.870 \\
\hline
\end{tabular}
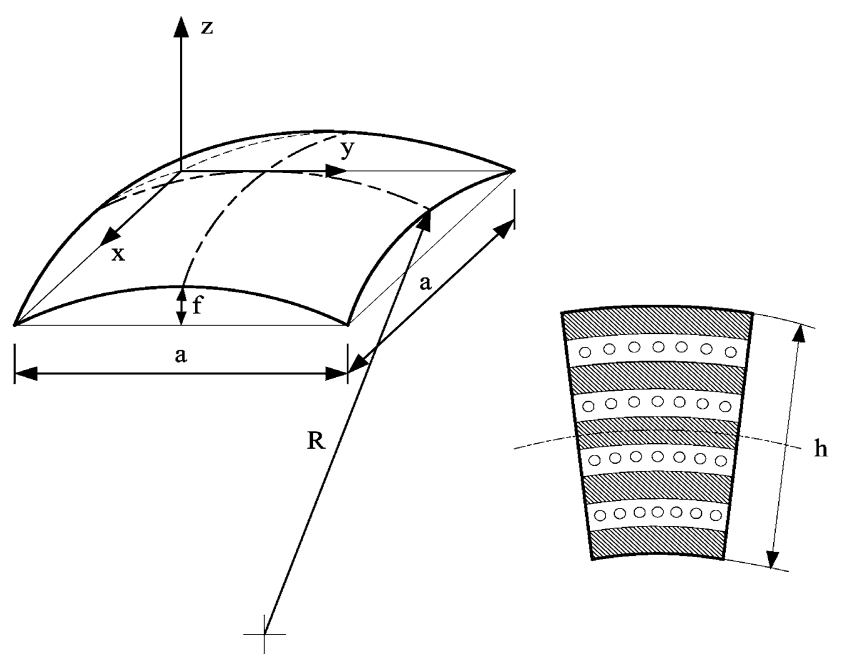

Fig. 23 Laminate composite hinged spherical shell

conforming solution by Voyiadjis and Shi [135] as can be shown in Fig. 29.

Hinged Spherical Shell with Concentrated Load The hinged spherical shell problem is also a snap-though problem. Its added complication is the curvature in two directions. The geometric properties are radius $R=2540.0 \mathrm{~mm}$ and length $a=784.5 \mathrm{~mm}$. It is hinged on all sides and is loaded with a point load at the center. The modulus of elasticity $E=68.95 \mathrm{kN} / \mathrm{mm}^{2}$ and Poisson's ratio $v=0.30$.

The problem is modeled with a $3 \times 3$ mesh of one quarter of the shell. The general behavior using XSHELL41 is in agreement with the references by Horrigmoe and Bergan [62] and Dhatt [36]. Horrigmore and Bergan's solution used flat triangular elements and Leicester [84] provided a series solution.

Hemispherical Shell with 18-Degree Hole The hemispherical shell with an $18^{\circ}$ hole is a popular linear benchmark test to demonstrate an elements ability to solve problems involving, almost, inextensional bending. Its nonlinear analysis adds large displacements and rotations to the problem.

Taking advantage of symmetry, only one quarter of the hemisphere is modeled with $16 \times 16$ mesh size. The loaddeflection curves of present solutions are in good agreement with the published results of Buechter and Ramm [25], even with a course meshes.

Imperfect Rectangular Plate Under In-Plane Loading A thin, simply supported plate subjected to in-plane compressive loading is analyzed. The analysis includes both material and geometric non-linearity. The geometry and material properties are shown in Fig. 35. The initial out-of-plane imperfection is $w=w_{o} \sin (\pi x / a) \sin (\pi y / b)$. Because of symmetry, only one quarter of the plate is used in the model; $4 \times 4$ meshes for the XSHELL41. The tolerance for convergence used is 0.001 . Fifteen displacement increments are used with a maximum of 4 iterations for the three-node and 3 iterations for the four-node of MNR per increment. The reference solutions are by Javaherian and Dowling [73]. They used a $4 \times 4$ mesh of semi-loof elements. A tolerance of 0.001 was used, 15 displacement increments were also made with a maximum of $5 \mathrm{MNR}$ iterations per increment. The stress-strain plot and stress-deflection plot trends are in good agreement. The spread of plasticity is also the same, starting from the center and plate corners and spreading diagonally. However, for the present results, it is seen that the top portion of the center returns to elasticity, which is due to the tension because of the bending of the plate. Also included are results from an 8 -node assumed strain shell element, XSHELL81 [81].

Large-Deflection Elasto-Plastic Analysis of Stiffened Plate Under In-Plane Loading A stiffened imperfect plate is subjected to compressive loading. The plate has three 
Table 16 Non-dimensional displacements of the laminated spherical shell

\begin{tabular}{|c|c|c|c|c|c|}
\hline \multirow[t]{2}{*}{$h / a$} & \multirow{2}{*}{$\begin{array}{l}\text { Quarter } \\
\text { mesh }\end{array}$} & \multicolumn{2}{|c|}{$(0 / 90 / 0 / 90 / 0 / 90 / 0 / 90 / 0)$} & \multicolumn{2}{|c|}{$(45 /-45 / 45 /-45 / 45 /-45 / 45 /-45 / 45$} \\
\hline & & XSHELL41 & $\begin{array}{l}\text { Noor \& Mathews } \\
\text { (1975) [97] Analytical }\end{array}$ & XSHELL41 & $\begin{array}{l}\text { Noor \& Mathews } \\
\text { (1975) [97] FEM }\end{array}$ \\
\hline \multirow[t]{2}{*}{0.1} & $8 \times 8$ & $4.931 \mathrm{E}-03$ & $5.797 \mathrm{E}-03$ & $3.371 \mathrm{E}-03$ & $3.540 \mathrm{E}-03$ \\
\hline & $10 \times 10$ & $5.084 \mathrm{E}-03$ & & $3.544 \mathrm{E}-03$ & \\
\hline \multirow[t]{2}{*}{0.01} & $8 \times 8$ & $2.695 \mathrm{E}-03$ & $2.717 \mathrm{E}-03$ & $5.326 \mathrm{E}-04$ & $5.170 \mathrm{E}-04$ \\
\hline & $10 \times 10$ & $2.701 \mathrm{E}-03$ & & $5.337 \mathrm{E}-04$ & \\
\hline \multirow[t]{2}{*}{0.001} & $8 \times 8$ & $5.895 \mathrm{E}-05$ & $5.916 \mathrm{E}-05$ & $-1.012 \mathrm{E}-05$ & $-1.050 \mathrm{E}-05$ \\
\hline & $10 \times 10$ & 5.911E-05 & & $-1.054 \mathrm{E}-05$ & \\
\hline
\end{tabular}

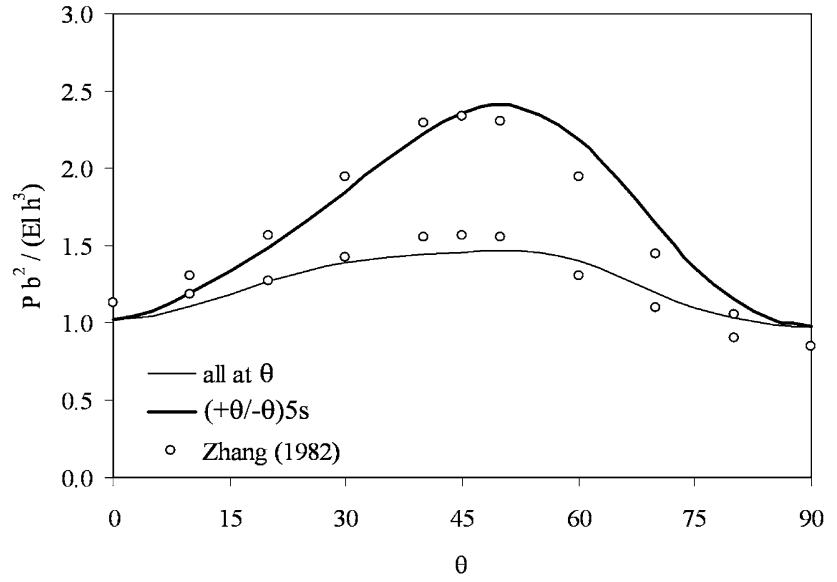

Fig. 24 Buckling eigenvalues of simple plate

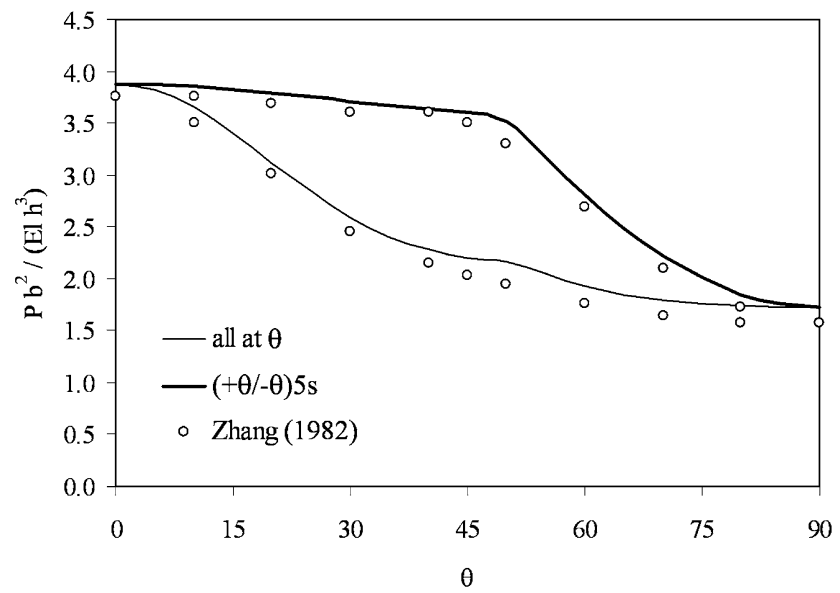

Fig. 25 Buckling eigenvalues of clamped plate

equally spaced stiffeners and has welded supports. The imperfection is created using a continuous function $w_{o}(x, y)$. Only half of the stiffened plate is used for the finite element model because of symmetry. For the XSHELL41 element, a mesh of $16 \times 12$ is made for the plate and $4 \times 12$ for the stiffeners. The geometry and material properties are shown in Fig. 38.

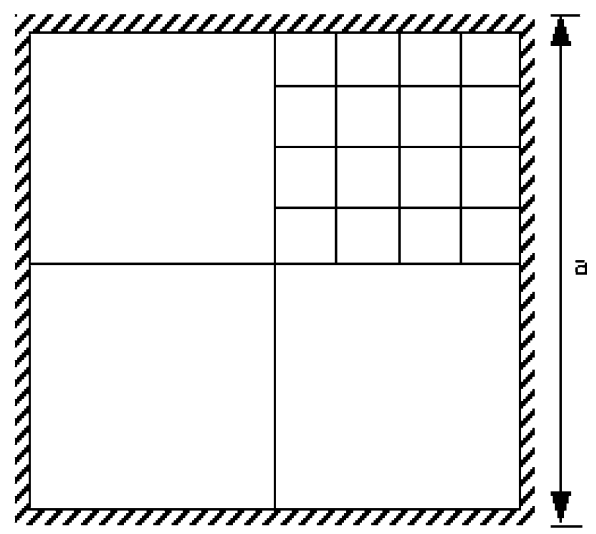

Fig. 26 Square clamped plate subjected to uniform pressureGeometry and mesh

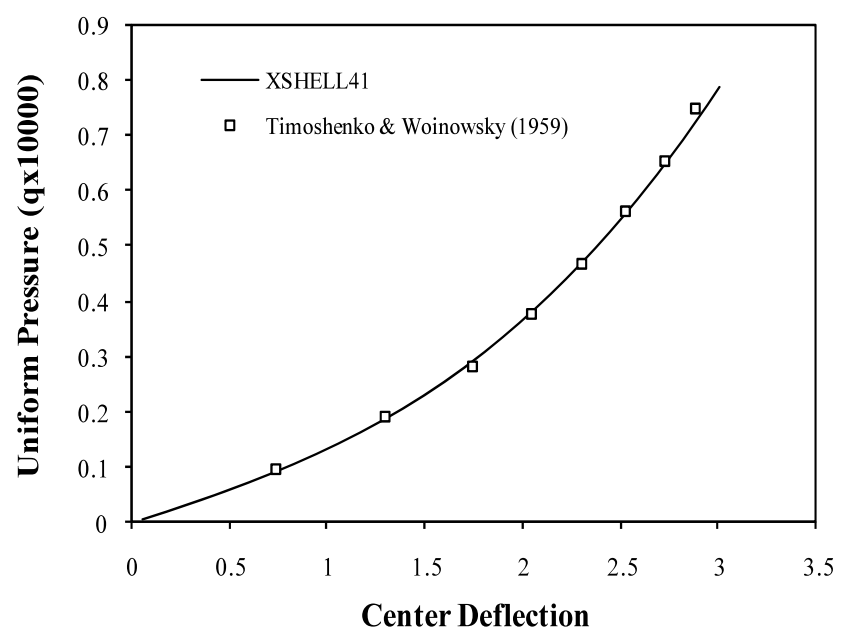

Fig. 27 Square clamped plate subjected to uniform pressure

For the elasto-plastic analysis, six layers are assumed in the thickness direction for both the plate and the stiffeners. No residual stress is applied. Material failure initially occurs at the center of the plate where the displacement is greatest. The spreading of plastic failure follows the stiffener connections, where the restraints cause greater stress. The limit 
Fig. 28 Hinged cylindrical

shell geometry and mesh

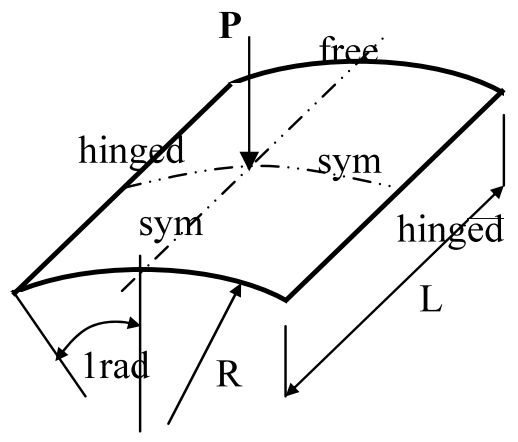

$$
\begin{aligned}
& \mathrm{R}=2540 \mathrm{~mm} \\
& \mathrm{~L}=508 \mathrm{~mm} \\
& \mathrm{~h}=12.7 \mathrm{~mm} \text { or } 6.35 \mathrm{~mm} \\
& \theta=0.1 \mathrm{rad} \\
& \mathrm{E}=3.10275 \mathrm{kN} / \mathrm{mm}^{2}
\end{aligned}
$$
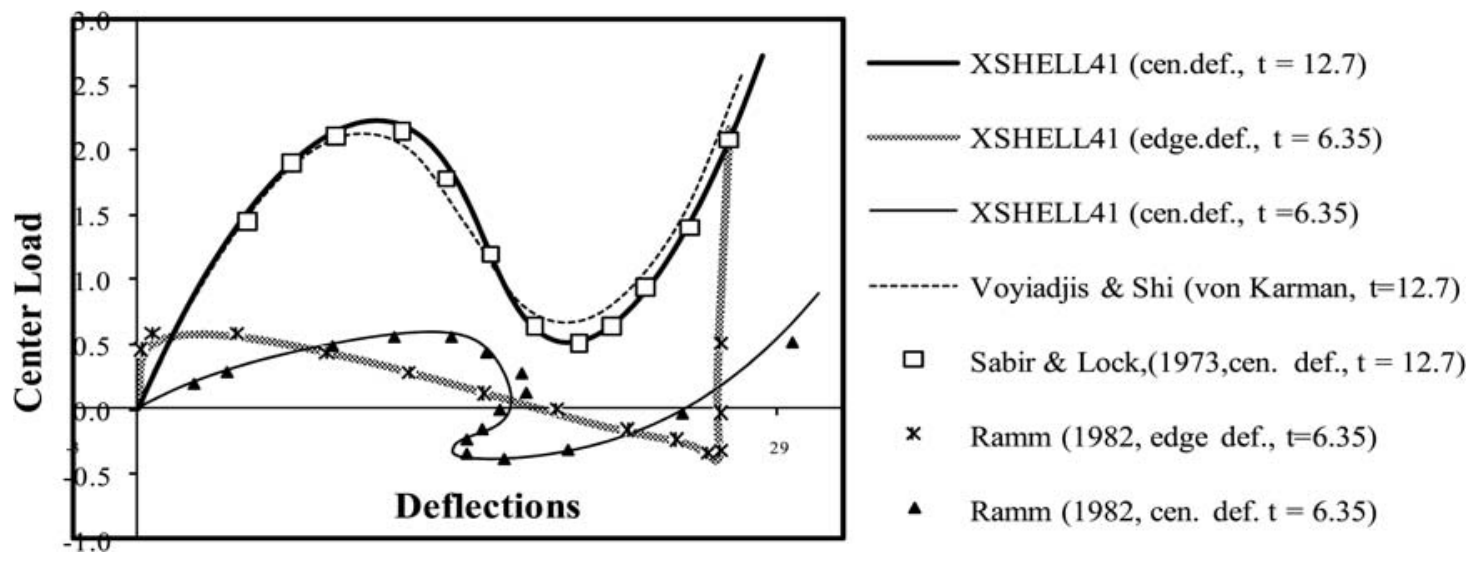

Fig. 29 Hinged cylindrical shell load-deflection curve

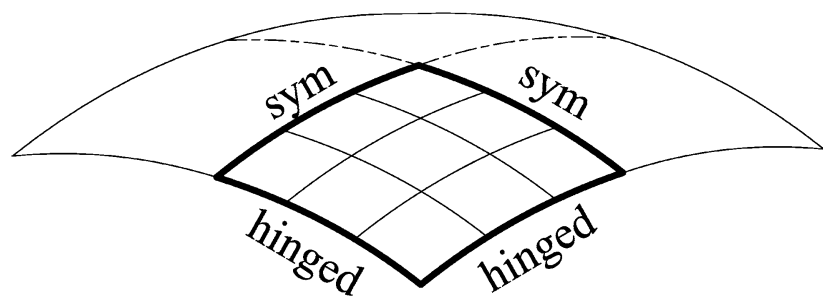

Fig. 30 Hinged spherical shell geometry and mesh

load is reached when almost the entire width of the plate is plastic and the stiffeners start to fail.

The load-shortening curve obtained is shown in Figs. 3941, including the numerical studies of Webb and Dowling [166] and Djhani [38]. The reference numerical analysis used finite difference method. A full depth yield criterion for the plate was used, while stiffeners were of multi-layer approach. The result of an actual test indicates a normalized peak load of 0.835 . The difference between the present analysis and the test results may be attributed to the difference in residual stresses and actual imperfections of the specimen.

Elasto-Plastic Buckling of Imperfect Stiffened Curved Panel Agelidis [1] carried out parametric studies of stiffened cylindrical shells covering a broad range of geometries used in the offshore structural engineering. The studies were carried out using only a part/panel of the cylinder. In the following, an imperfect stiffened Agelidis panel subjected to uniform inplane compressive loading is analyzed. In the analysis, it is assumed that the panel undergoes large deformation and material non-linearity. The panel is $1 / 20$ of a cylinder and has a stiffener at the middle. The ends of the cylinder are bounded by ring stiffeners, thus are assumed to be fixed against displacement. The initial imperfections are made by assuming a sine wave along the length and half a sine wave in the circumferential direction. The maximum imperfection amplitude is 0.411 . Only one half of the stiffened panel is used for the model because of symmetry. The same mesh is used for both the panel and the stiffener, $4 \times 6$ XSHELL41 elements.

The plot of the applied load vs. axial shortenning is shown in Fig. 41. The plot also shows the result using XSHELL81 [81]. The results show a very sharp limit point and a snap-back behaviour with the sudden drop in load carrying capacity. The axially loaded cylinder is extremely sensitive to imperfections, which is also shown in Fig. 41. Increasing the imperfection amplitude by $50 \%$ decreases the limit load by $20 \%$. It can also be observed that the postbuckling behaviour is unchanged, and is influenced by the yield strength. The maximum post buckling capacity would be reached once plasticity has spread throughout the cylinder. Thus, the limit point is dictated by the initial imperfec- 
Fig. 31 Hinged spherical shell load-deflection curve

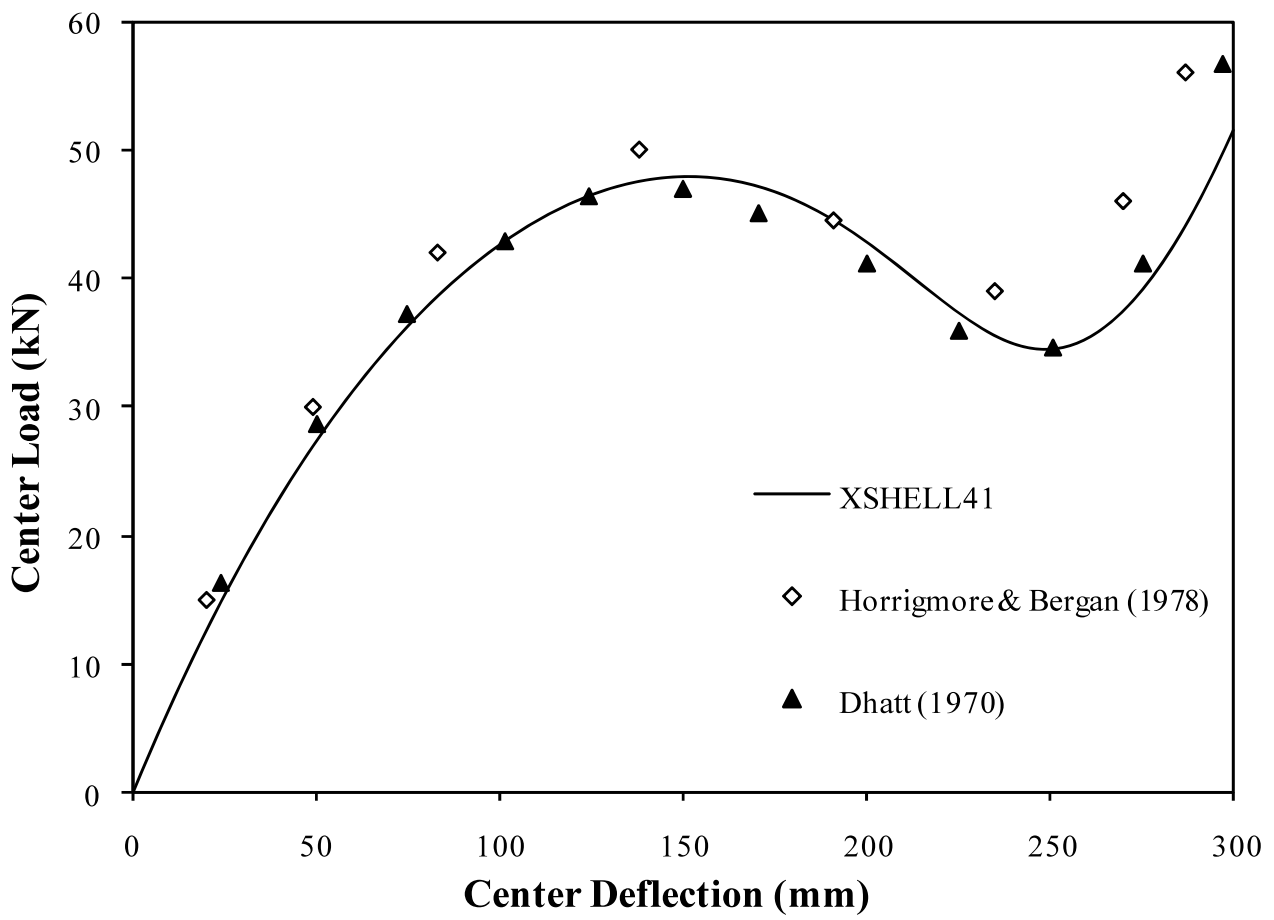

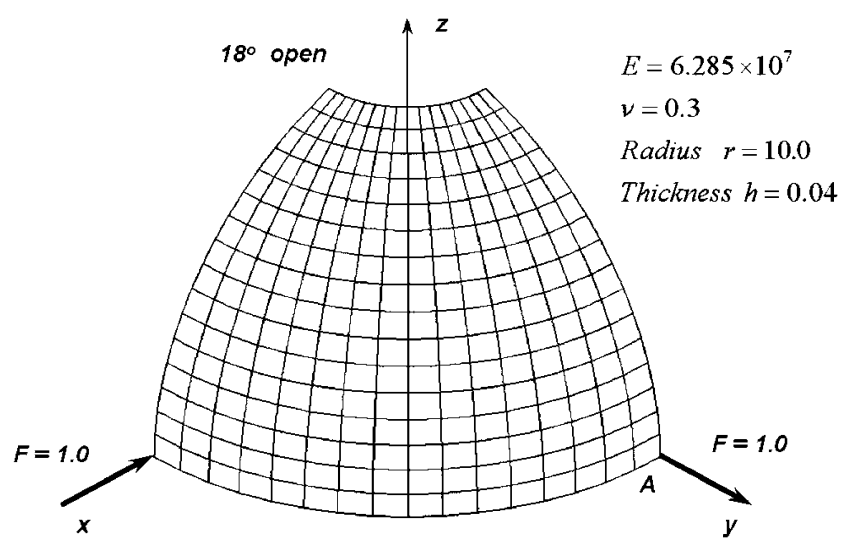

Fig. 32 Hemispherical shell with $18^{\circ}$ hole mesh

tion and the maximum post-buckling capacity depends on the yield strength.

Pinched Elasto-Plastic Cylinder with Isotropic Hardening A thin cylinder, pinched at the mid sections is analyzed for large displacement, rotation and elasto-plastic behavior. von Mises yield criterion with isotropic hardening is used with six stations along the thickness. The cylinder has two end diaphragms. It is modeled using only one octant with $32 \times$ 32 XSHELL41 elements.

The cylinder geometry and material properties are shown in Fig. 43. The solution by Simo and Kennedy [137] using generalized Ilyushin-Shapiro elastoplastic model and Brank et al. [23] using von Mises yield criterion with seven integration points are also shown with the present results, Fig. 44.
The same snap-through response observed by Brank et al. is also seen in the present analysis with similar limit points and stiffening behavior. The geometry at different stages of deformation is shown in Fig. 45.

Collapse of Scordelis-Lo Roof The Scordelis-Lo roof is a popular benchmark problem for linear analysis. The inclusions of geometric and material non-linearity, with large rotations are complicating factors. The roof has a half-length of $L=7.6 \mathrm{~m}$, radius of $R=7.6 \mathrm{~m}$, with a half-angle of 40 deg across. It is $t=0.076 \mathrm{~m}$ thick, subjected to a load, proportional to its own weight, $w t=4.0 \mathrm{kN} / \mathrm{m}^{2}$, and supported by rigid diaphragms at its ends. The material properties are $E=2.1 \mathrm{e} 4 \mathrm{~N} / \mathrm{mm}^{2}, v=0$ and $\sigma_{y}=4.2 \mathrm{~N} / \mathrm{mm}^{2}$. Due to symmetry, only one quarter of the roof with a mesh of $32 \times 32$ XSHELL41 elements is used in the analysis model.

Many authors had performed the non-linear analysis, like Peric and Owen [107], Crisfield and Peng [33], Brank et al. [23], and Roehl and Ramm [122]. Roehl and Ramm used solid and shell elements while the others used only shells. Plotting the load-deflection curves of point $A$, two solution trends seem to be available. Type one is with a sudden drop in load carrying capacity, and the other, type two is where there is a less drastic loss of load capacity with a gain in stability as the deflection increases. Roehl and Ramm also presented cases where, using shell elements under a small strain assumption, the results vary in trend, depending on the number of stress stations through the thickness. This is also experienced for the present four-node formulation. Using three stations along the thickness, type one trend is experienced, 
Fig. 33 Hemispherical shell with $18^{\circ}$ hole plot

Fig. 34 Deformed shape of hemispherical shell with 18 degree hole

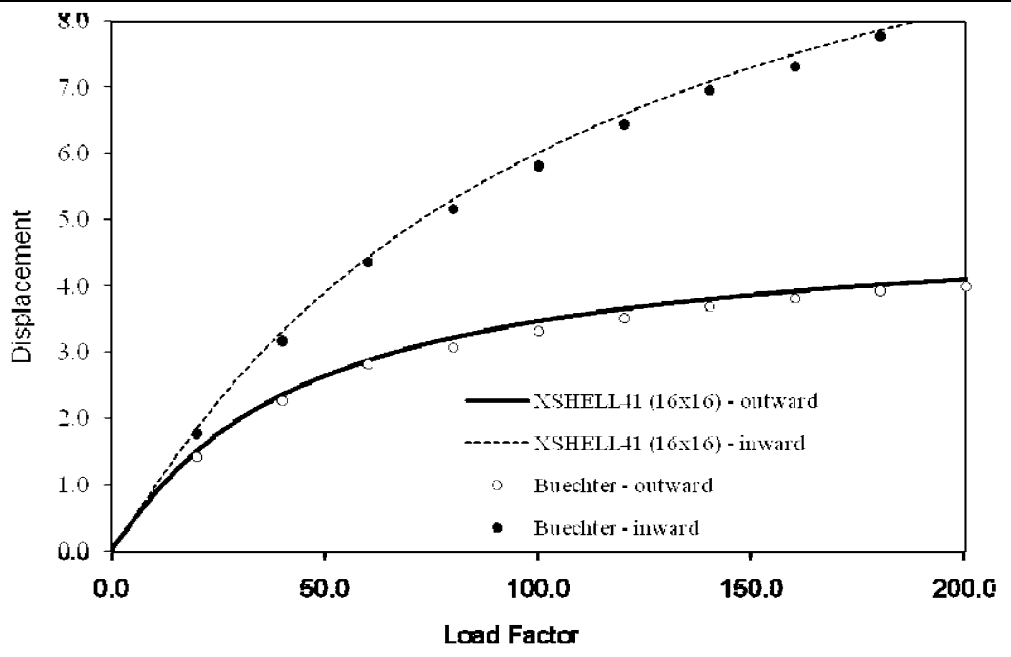

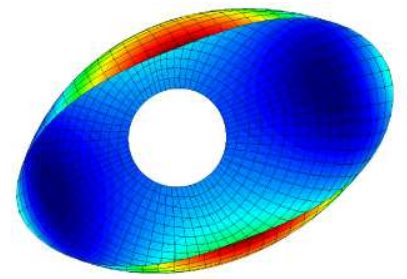

Disp. Ay $=2.523$

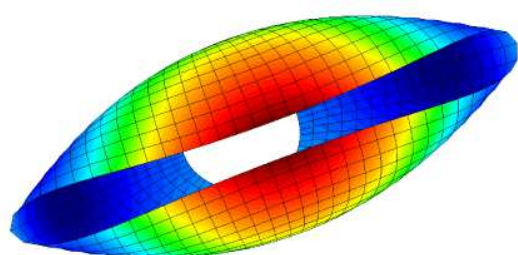

Disp. Ay $=4.293$

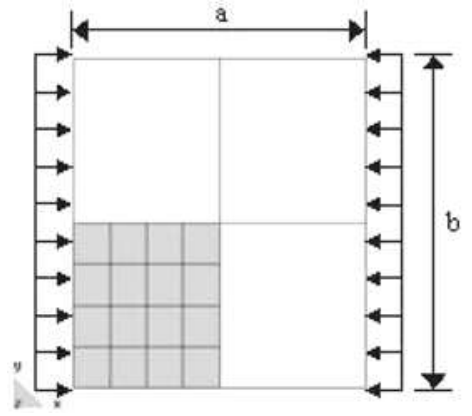

$a b=0.875$

$b i t=80$

$\mathrm{t}=0.003175 \mathrm{~m}$

$\mathrm{E}=2.062 \mathrm{e} 11 \mathrm{~N} / \mathrm{mm}^{2}$

$y=0.3$

$\sigma \mathrm{y}=2.50 \mathrm{e} 8 \mathrm{~N} / \mathrm{mm}^{2}$

$w_{0}=0.001 b$ at the center
Fig. 35 Plate geometry and material properties

while type two for five and seven stations. Type one is a result of the formation of hinges at the top off the structure. The present element has difficulty forming these hinges because of the assumption of a constant thickness throughout the deformation. For this problem, the importance of thickness deformation is emphasized.

\subsection{Nonlinear Laminate Composite Problems}

Hinged Cylindrical Shell under Point Load The snapthrough behavior of a hinged cylindrical shell under a center point load is analyzed. The geometric and material properties of the shell are shown in Fig. 49. Because of symmetry only one quarter is modeled using an $8 \times 8$ mesh of XSHELL41 element. Two lay-ups are studied, (45/-45) and
$(0 / 90 / 0)$ and the results are plotted with those obtained by XSHELL81 [81] and Saigal et al. [127]. The present element gives a lower limit load than the reference but give generally the same behavior.

Boron-Epoxy Panel Under In-plane Loading A flat and curved panel under in-plane loading is analysed for their post buckling behaviour. The panel geometry and material properties are shown in Fig. 51. Two types of lay-ups are used, (90/0) and (45/-45)s. For the angle-ply lay-up, an initial imperfection of

$\bar{w}=0.5 \sin \left(\pi x / L_{x}\right) \sin \left(\pi y / L_{y}\right)$

was used. $16 \times 16$ elements were used to model both problems and the results are shown with the analytical solution provided by Zhang [181] and the FE solution from XSHELL81 [81]. While the general trends of the results are the same as those given by Zhang, the curve panel solutions tend to give higher buckling loads.

\section{C4/Graphite-Epoxy Panel Under In-plane Compressive} Loading Starnes and Rouse [147] conducted experimental studies on the post buckling and failure characteristics of a C4/graphite-epoxy panel, T300/5208. The panel consists of 24 plies, $\left( \pm 45 / 0_{2} / \pm 45 / 0_{2} / \pm 45 / 0 / 90\right)_{\mathrm{s}}$, and is $508 \mathrm{~mm}$ high, $178 \mathrm{~mm}$ wide and $3.36 \mathrm{~mm}$ thick. The typical lamina properties are 
Fig. 36 (a) Normalized average in-plane stress vs. Average in-plane strain - 4 node. (b) Normalized force vs. out-of-plane displacement diagram -4 node

Fig. 37 Development of top layer plasticity, dark areas are plastic zones

Fig. 38 Stiffened plate geometry and material

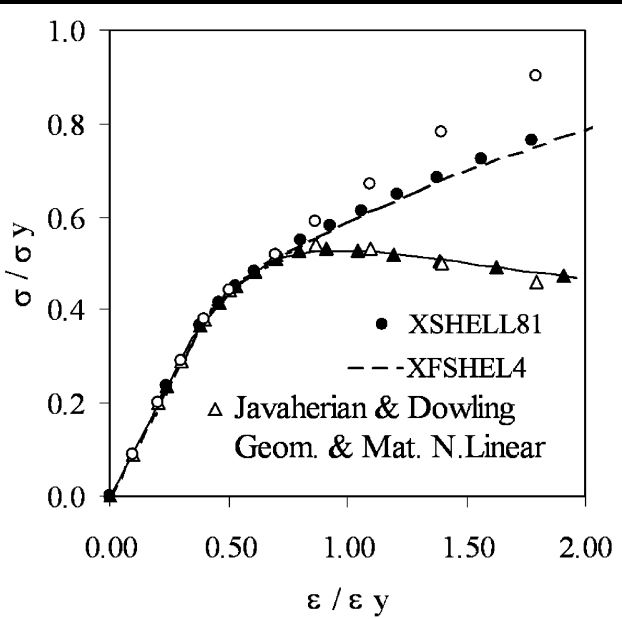

(a)

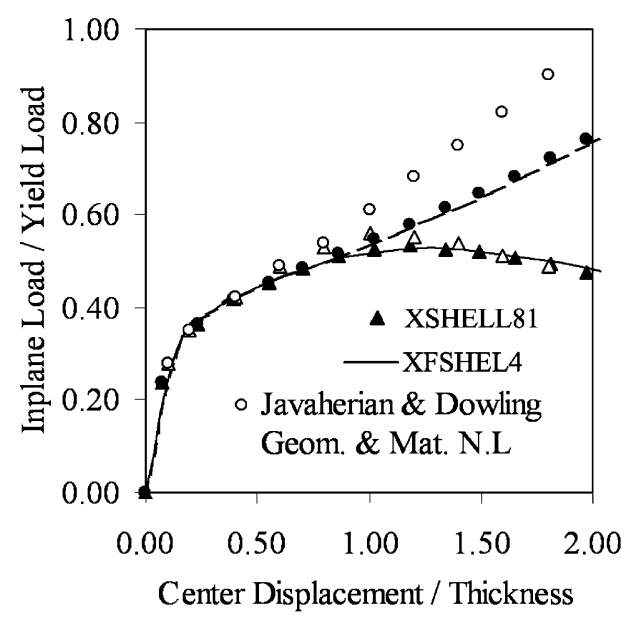

(b)

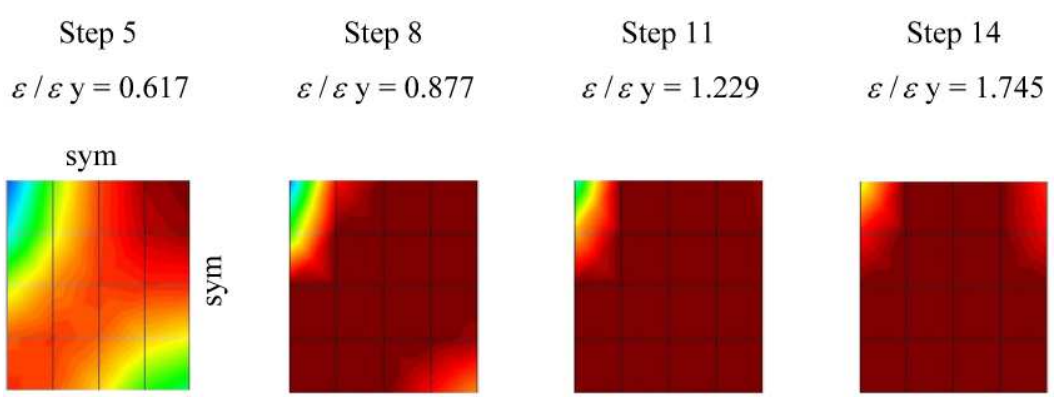

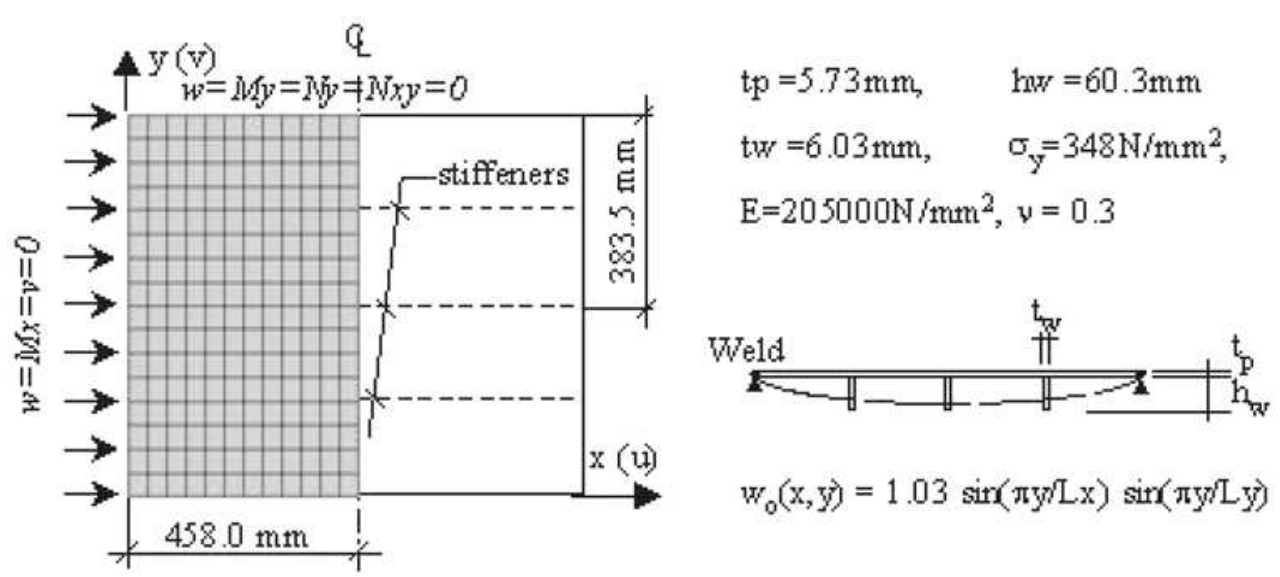

\begin{tabular}{|c|c|c|c|c|c|}
\hline \multicolumn{4}{|c|}{ Elastic Constants } & \multicolumn{2}{|c|}{ Strengths } \\
\hline$E_{1}$ & $131,000 \mathrm{Mpa}$ & $v_{12}$ & 0.38 & $\mathrm{XT}$ & 1,400 Mpa \\
\hline$E_{2}$ & $13,000 \mathrm{Mpa}$ & $v_{31}$ & 0.38 & $\mathrm{XC}$ & 1,138 Mpa \\
\hline$E_{3}$ & $10,000 \mathrm{Mpa}$ & $v_{23}$ & 0.38 & YT & $80.9 \mathrm{Mpa}$ \\
\hline$G_{12}$ & 6,400 Мра & & & $\mathrm{YC}$ & $189 \mathrm{Mpa}$ \\
\hline$G_{31} *$ & 3,200 Мра & & & Q & $69 \mathrm{Mpa}$ \\
\hline$G_{23} *$ & 6,400 Мра & & & & \\
\hline layer $\mathrm{t}$ & ness $0.14 \mathrm{mn}$ & & & & \\
\hline
\end{tabular}

The loaded ends were clamped and the unloaded edges were simply supported to prevent the panel from buckling as a wide column. In actual tests, $\mathrm{C} 4$ panels with these conditions were observed to buckle into two longitudinal half sine waves and one transverse half sine wave. The boundary conditions were: at $x=0,178, W_{0}=X_{0}=0$, at $y=0$, $U_{0}=W_{0}=X_{0}=d W / d x=0$, at $y=508, W_{0}=X_{0}=$ $d W / d x=0, U_{0}=$ constant. The analytical buckling load and critical end shortening from [147] is $40 \mathrm{kN}$ and $0.5 \mathrm{~mm}$, respectively. 
Fig. 39 Normalized average in-plane stress vs. average in-plane strain of stiffened plate
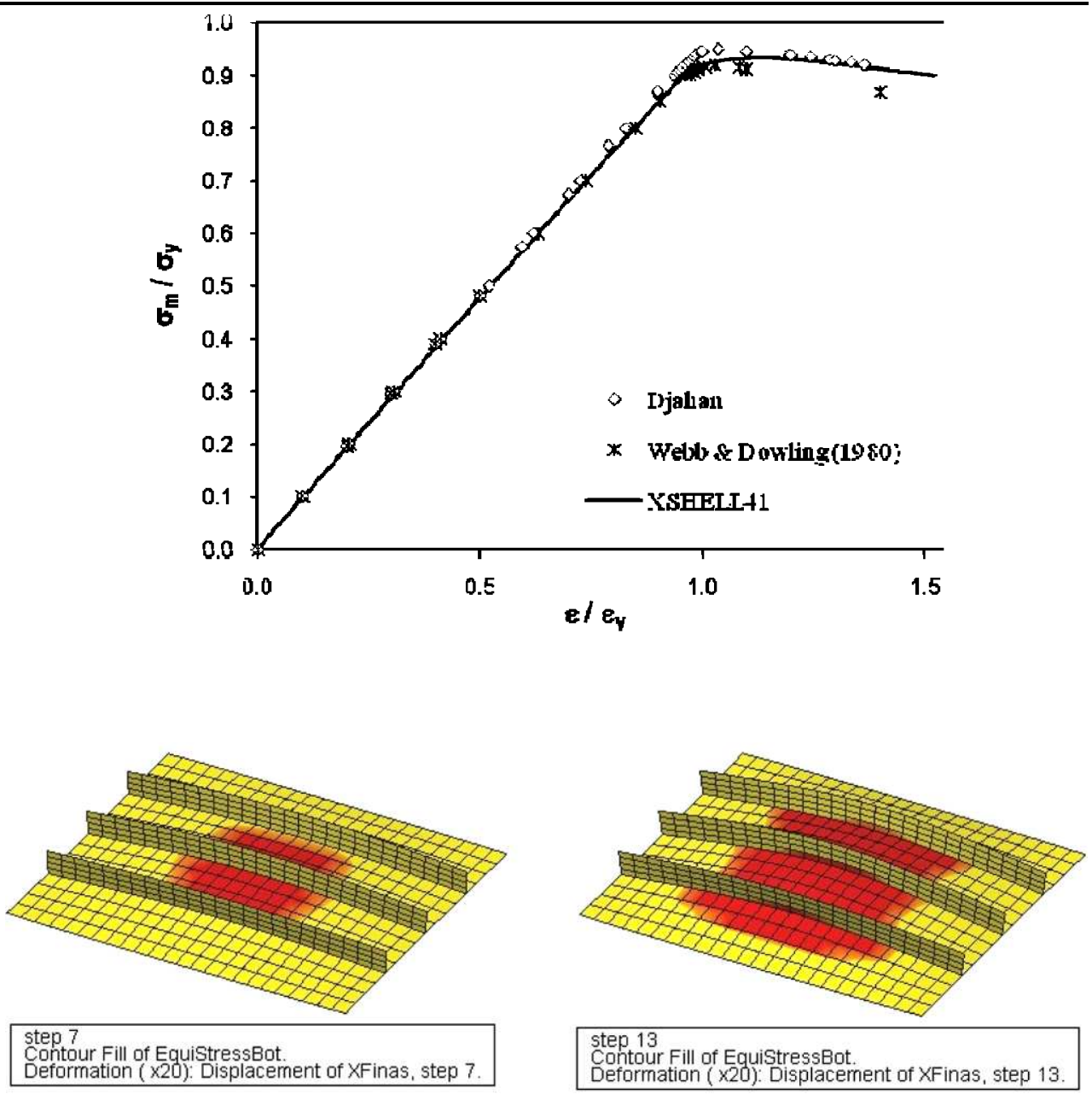

Onset of plasticity (x20) - point a

Limit point shape $(\mathrm{x} 20)-$ point $b$

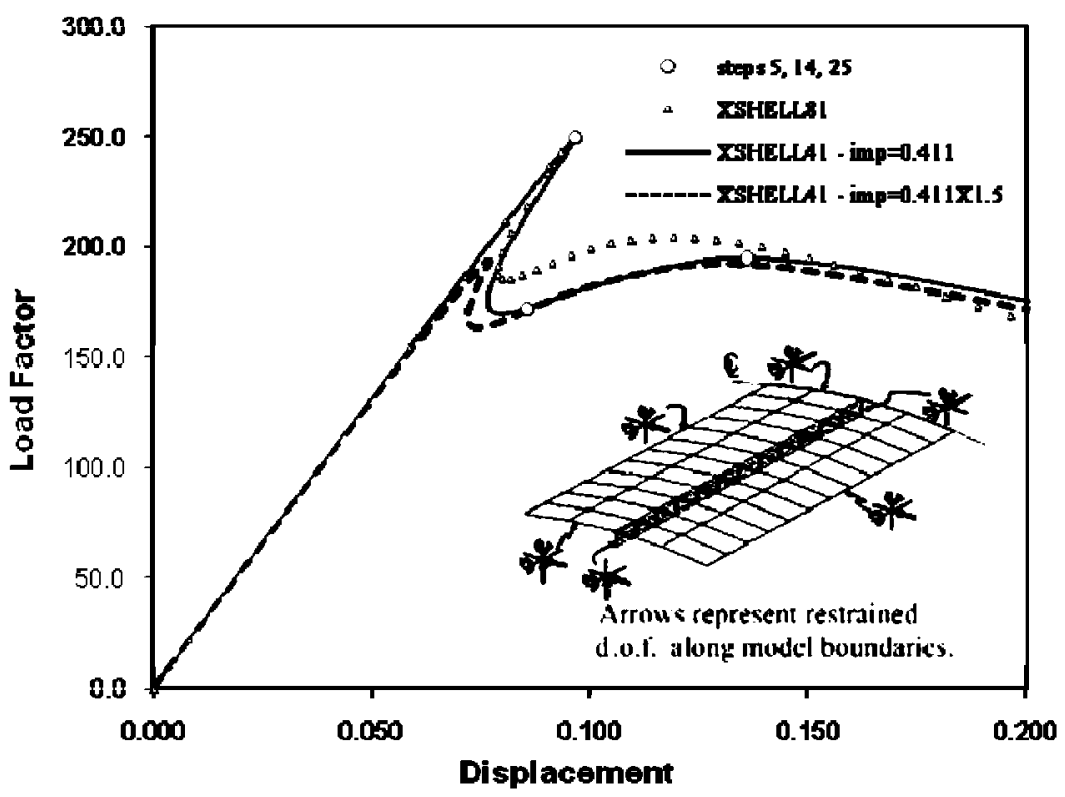

Geometry Properties

Panel
Length $=160.0 \mathrm{~mm}$
Radius $=160.0 \mathrm{~mm}$
thickness $=0.63 \mathrm{~mm}$

Stiffener

Length $=160.0 \mathrm{~mm}$

Depth $=3.6 \mathrm{~mm}$

thickness $=0.63 \mathrm{~mm}$

Material Properties

$\mathrm{E}=210000.0 \mathrm{Mpa}$

$\mathrm{v}=0.3$

$\sigma_{\mathrm{y}}=365.0 \mathrm{Mpa}$

Fig. 41 Stiffened curved panel load-deflection curve 
Fig. 42 Deformed shapes $(\times 5)$ and spread of plasticity of an imperfect stiffened cylinder under axial compression, dark areas are plastic zones

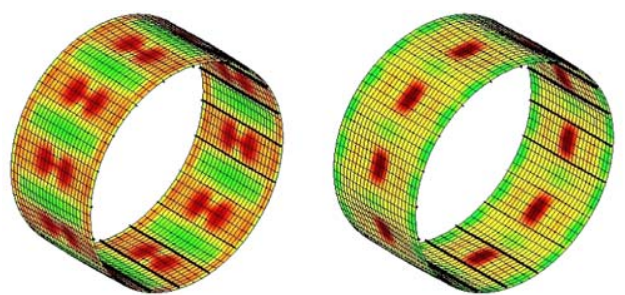

Step 5 - Limit Point
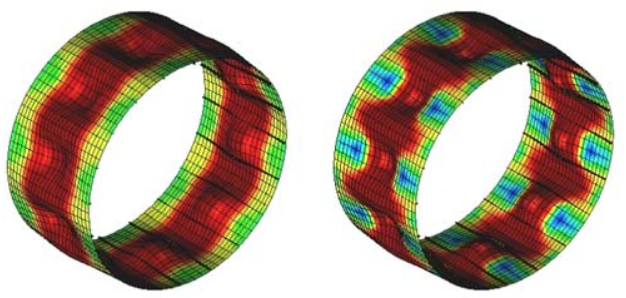

Step 25 - Max Point at Post Buckling

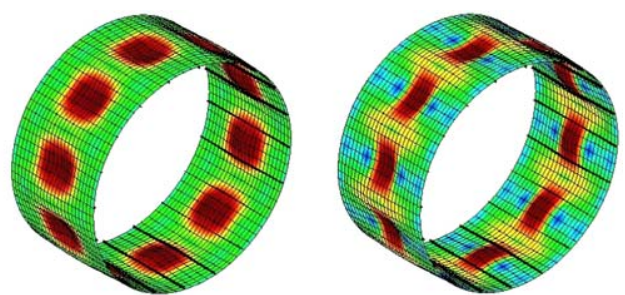

Step 14 - Min Point at Post Buckling
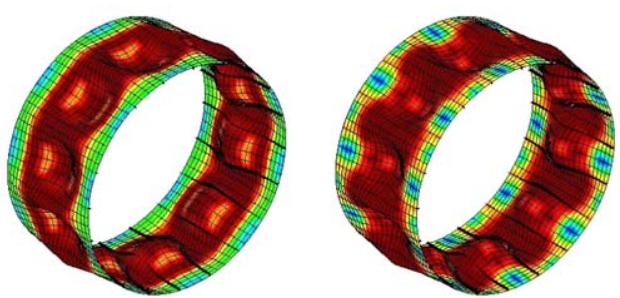

Step 40 - End of Analysis

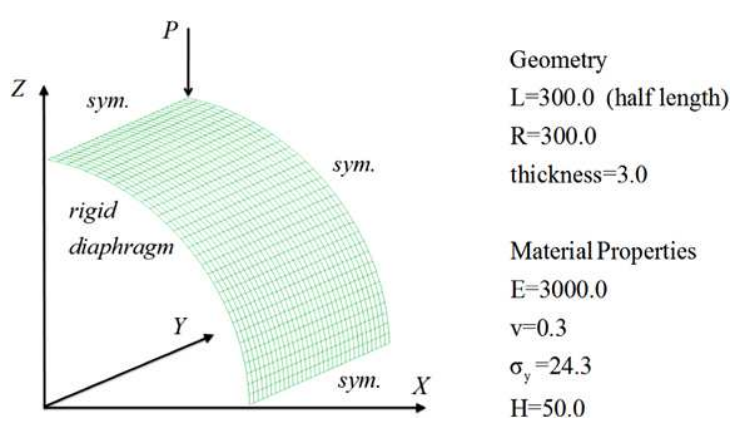

Fig. 43 Pinched elasto-plastic cylinder-geometry and material properties

The panel is modelled using XSHELL41 elements, 12 along the width and 24 along the height. An initial imperfection with amplitude of $1 \%$ of the plate thickness is assumed to obtain an equilibrium path beyond the critical buckling point. The imperfection is given in the following form:

$w_{o}=0.0336 \sin \left(\pi x / L_{x}\right) \sin \left(\pi y / L_{y}\right)$.

The results of the numerical analysis for the failure of the $\mathrm{C} 4$ panel are plotted in Fig. 55. It is a plot of the end shortening normalized with the critical end shortening $u_{c r}=$ $0.5 \mathrm{~mm}$ and the applied load normalized by the analytical buckling load $P_{c r}=40 \mathrm{kN}$.

Three types of failure criteria are used in the analysis, maximum stress, Tsai-Wu and Tsai-Hill criterion. The complete matrix failure in an element dictates the failure for the numerical analysis. The finite element model provides a very close fit to the experimental results in both the pre and post buckling range. The buckling point is also very well defined. Among the three failure criteria used, the Tsai-Wu has the closest match to the experimental failure value. All panel failure values obtained in the numerical analysis are higher than the reference result.
E-glass Plate Under Transverse Loading Moy et al. [95] experimentally studied fiber-reinforced plastic panels under transverse loading. A clamped laminated plate made of e-glass/polyester type $(0 / 45 / 90 /-45 / 0)$, referred from their study is analyzed using the present XSHELL41 element with the different failure criteria. A full plate mesh of $16 \times 16$ elements is used and the results with those obtained by Moy et al. [95] are shown in Fig. 57. Loss of stiffness is shown on the behavior of the plate as a result ply failure. The layer-damaged region of the numerical analysis gives good approximation of the actual behavior of the plate.

Non-linear Dynamic Analysis of Composites with Layer Failure An E-glass plate the same as the previous problem is analyzed considering large deformations and dynamic effects. A uniformly distributed step load, $q=0.015 \mathrm{MPa}$ and $q=0.05 \mathrm{MPa}$ is applied. The plate mass density is $\rho=2.0 \mathrm{e}-6 \mathrm{Ns}^{2} / \mathrm{mm}^{4}$.

Equilibrium iterations and ply failure checking are performed with every time increment. For the larger load, more damage is incurred causing a significant change in stiffness. The time-central displacement plots are shown in Fig. 59.

Equilibrium iterations and ply failure checking are performed with every time increment. For the larger load, more damage is incurred causing a significant change in stiffness. The time-central displacement plots are shown in Fig. 59. The maximum positive displacement is approximately the same when laminate failure is considered and not considered. For the maximum negative displacement, the magnitude is significantly decreased.

\subsection{Reinforced Concrete Problems}

Unreinforced Concrete Cylinder under External Pressure This example is concerned with an unreinforced concrete 
Fig. 44 Pinched elasto-plastic cylinder-load-deflection curve

Fig. 45 Pinched elasto-plastic cylinder-deformed configuration

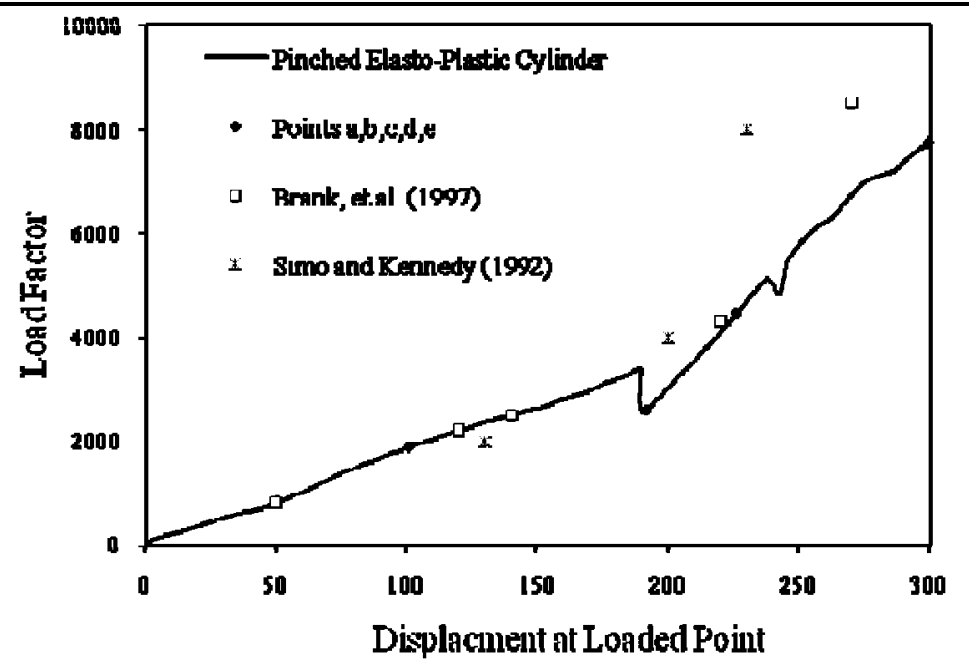

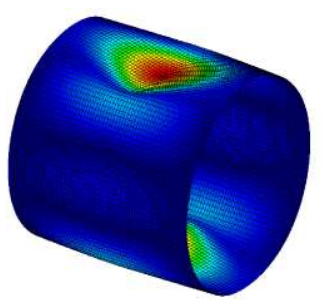

point a

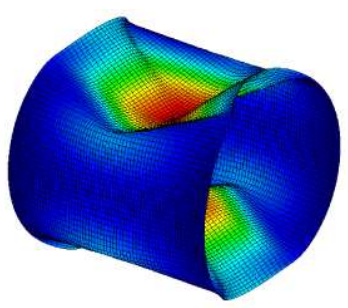

point c

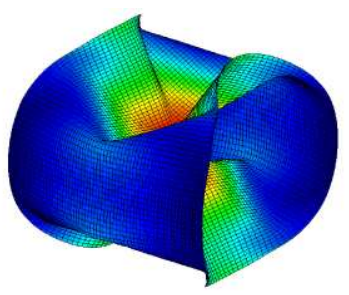

point e
Fig. 46 Scordelis-Lo roof, geometry, material properties and load

Fig. 47 Scordelis-Lo roof, load-deflection curves-4 node
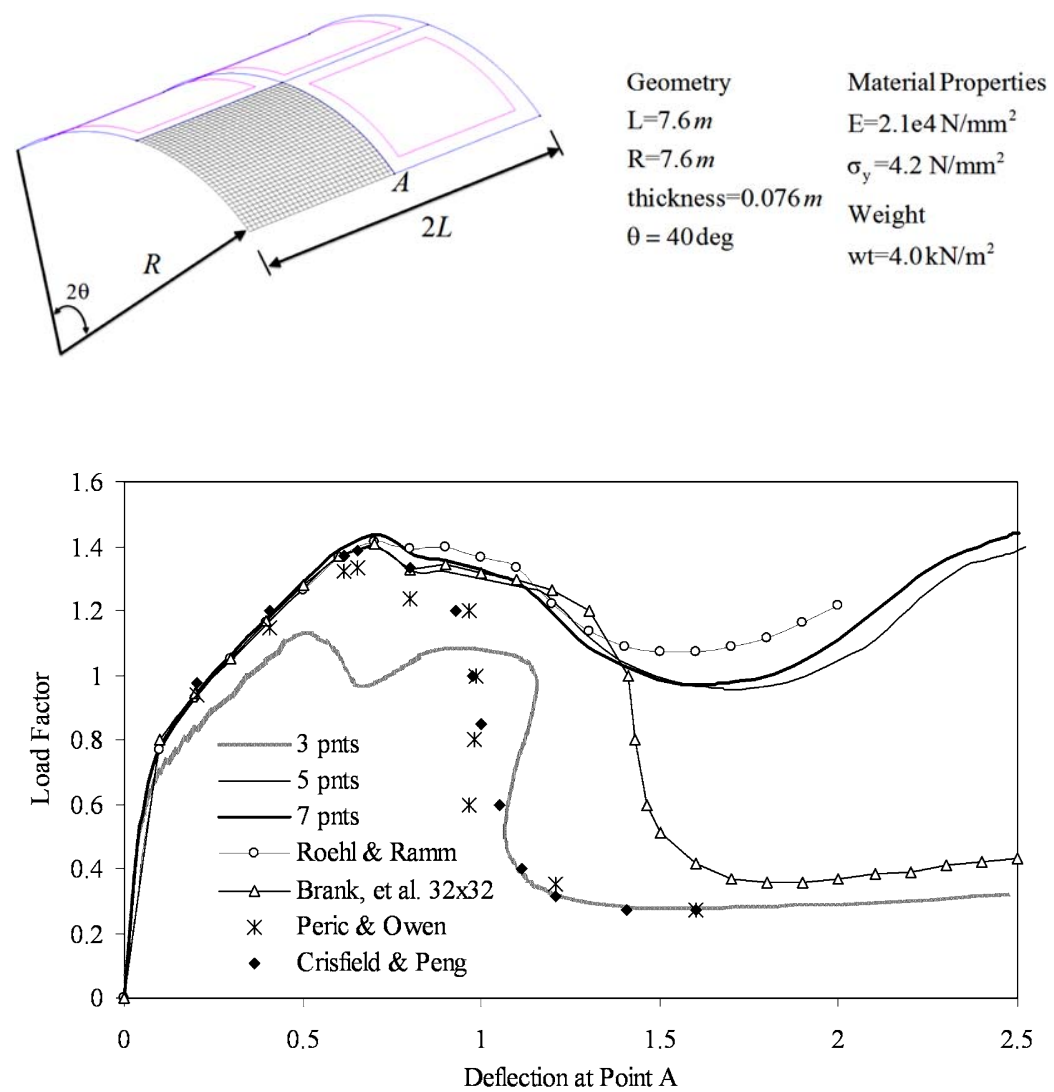
Fig. 48 Scordelis-Lo roof, final deformed shapes

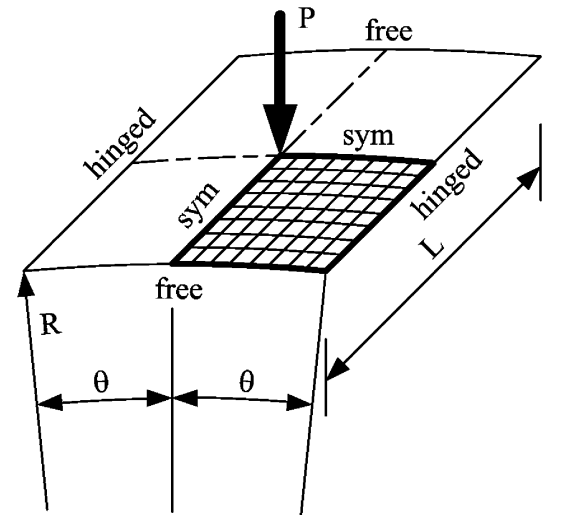

$\mathrm{R}=2540 \mathrm{~mm}$

$\mathrm{L}=508 \mathrm{~mm}$

$\mathrm{h}=12.7 \mathrm{~mm}$

$\theta=0.1 \mathrm{rad}$

$\mathrm{E}_{1}=3.3 \mathrm{kN} / \mathrm{mm}^{2}$

$\mathrm{E}_{2}=1.1 \mathrm{kN} / \mathrm{mm}^{2}$

$\mathrm{G}_{12}=0.66 \mathrm{kN} / \mathrm{mm}^{2}$

$v_{12}=0.25$

Fig. 49 Hinged cylindrical composite shell

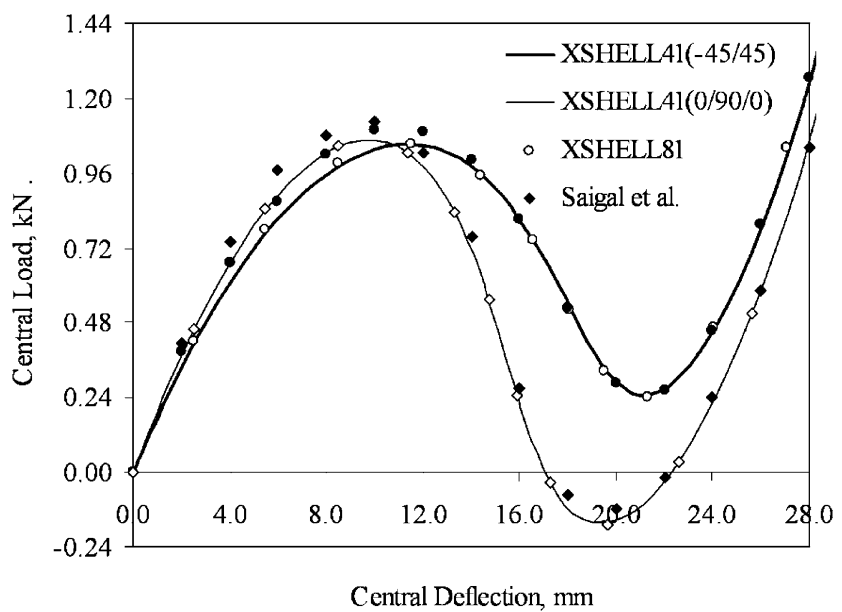

Fig. 50 Snap-trough behaviour of hinged cylindrical shell

cylinder subjected to an external water pressure until explosion occurs. The analyzed cylinder was part of an experimental test program conducted at the Naval Civil Engineering Laboratory in California, where 15 unreinforced concrete cylinders were put into a vessel loaded with external water pressure until failure. Under this condition, the biaxial compression stress will be produced everywhere in the cylinder wall and the performance of each concrete com-

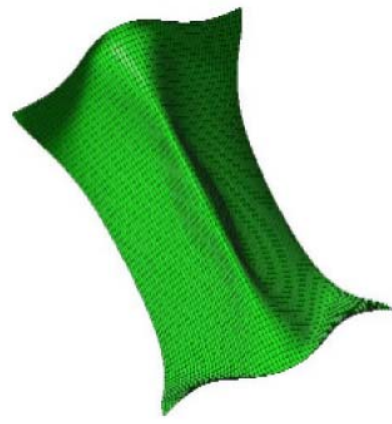

5 Stress points

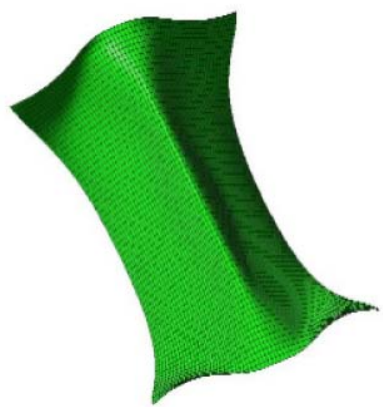

7 Stress Points pression models (Plasticity-based and EPF model) can be investigated. Runge and Haynes [125] reported the experimental results, while Chen et al. [29] conducted an independent analytical analysis at Purdue University. The input data for one of the analyzed cylinder is given in Fig. 61a.

The first analytical results were published by Chen and Chen [28] and Chang and Chen [29] who performed the analytical study, considering a plasticity-based model developed by Chen and Chen [28]. Later, Rule and Rowlands [124] analyzed one of those cylinders, shown in Fig. 61a, with a flat shell finite element which uses a linear shape function for in-plane displacements and a cubic polynomial for out of plane-displacement; they did not consider the geometric nonlinearity in their analysis. These authors adopted the orthotropic elastic model and the uniaxial strain approach proposed by Darwin and Pecknold [34] with a simplified biaxial failure envelope and a uniaxial stress strain curves for concrete.

The analysis of this example is performed considering the cylinder discretized as shown in Fig. 61b. By taking advantage of symmetry, only one octant of the cylinder is modeled with 150 elements. The cylinder thickness is divided into 10 equal layers. The material properties used are also shown in Fig. 61b. Results for the transverse mid-length displacement of the cylinder are plotted in Fig. 62. The average implosion pressure determine experimentally is $3.97 \mathrm{MPa}$, with a standard deviation of $0.17 \mathrm{MPa}$.

As shown in Fig. 62, the EPF model gives good agreement with the references, while plasticity-based model gives higher explosion pressure. The reasons for this difference are that the EPF model used the non-associate plasticity and it considers the fracture of concrete during the compression process, while the plasticity-based models do not. However, the trends of the load-displacement curves given by those models are similar.

Mcniece Slab An isotropically reinforced concrete slab, simply support at the four corners, was tested by Jofriet and Mcniece [76]. Many investigators had analyzed the same slab, among them were Lin [85] and Chung and Elisa [31]. 
Fig. 51 Geometry of panel under axial load

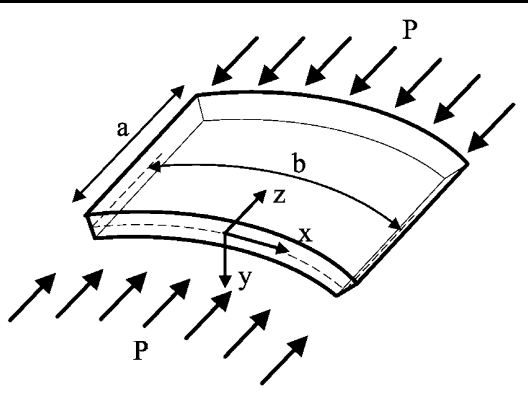

$$
\begin{aligned}
& \mathrm{a}=\mathrm{b}=250.0 \mathrm{~mm} \\
& \mathrm{~h}=2.50 \mathrm{~mm} \\
& \mathrm{E}_{1}=2.067 \mathrm{e} 5 \mathrm{~N} / \mathrm{mm}^{2} \\
& \mathrm{E}_{2}=2.07 \mathrm{e} 4 \mathrm{~N} / \mathrm{mm}^{2} \\
& \mathrm{G}_{12}=5.2 \mathrm{e} 3 \mathrm{~N} / \mathrm{mm}^{2} \\
& \mathrm{v}_{12}=0.30 \\
& \mathrm{~K}_{\mathrm{R}}=\mathrm{b}^{2} / \mathrm{R} \mathrm{h}=25 \text { (for curved Panel) }
\end{aligned}
$$

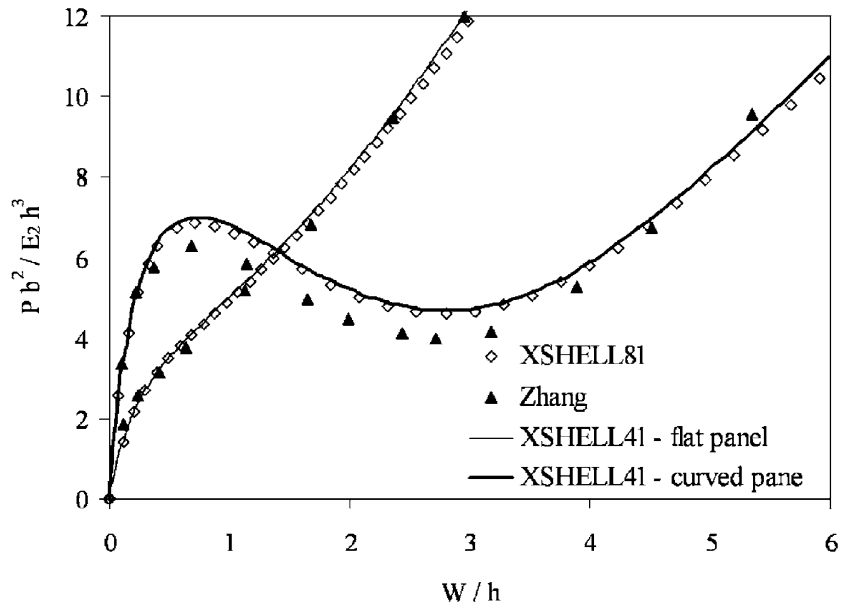

Fig. 52 Variation of central deflection with axial compression of boron-epoxy panels (90/0) lay-up

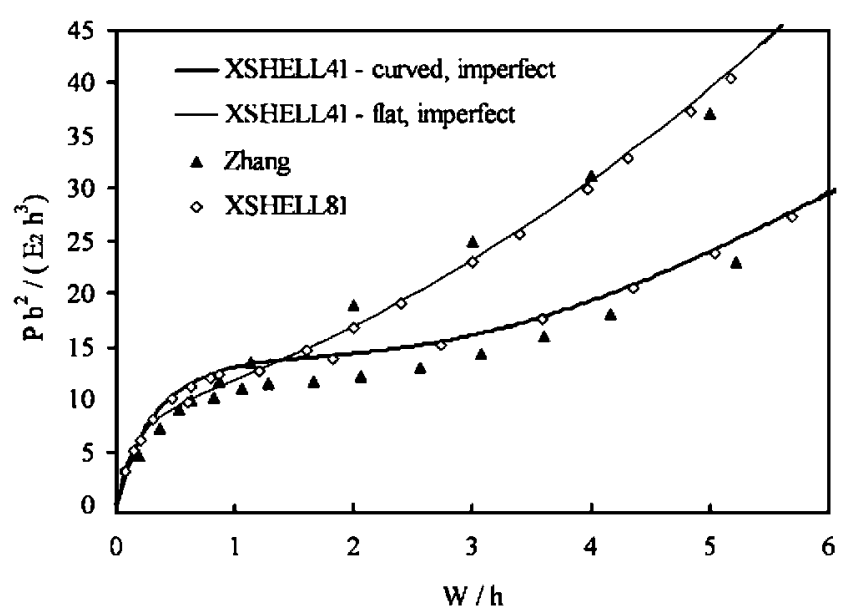

Fig. 53 Variation of central deflection with axial compression of imperfect boron-epoxy panels $(45 /-45)$ lay-up

The material properties of concrete and reinforcing bar are given in Table 1 . The geometry and reinforcement of the slab are shown in Fig. 63. Due to symmetry, one-quarter of the slab is modeled by the present shell element with a $6 \times 6$ mesh. The thickness of the slab is divided into 10 equal layers, and the reinforcement is represented by an equivalent smeared double layer with a $0.85 \%$ steel ratio. The point load is applied vertically at the center of slab. The trans-

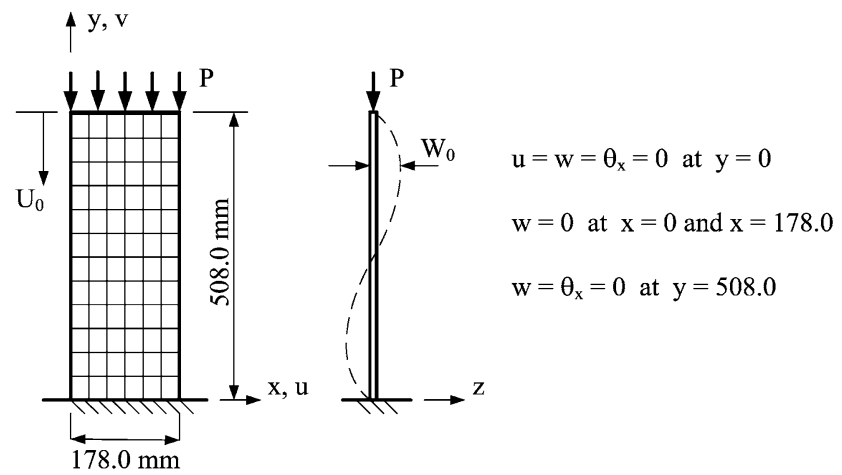

Fig. 54 C4/Graphite-epoxy panel

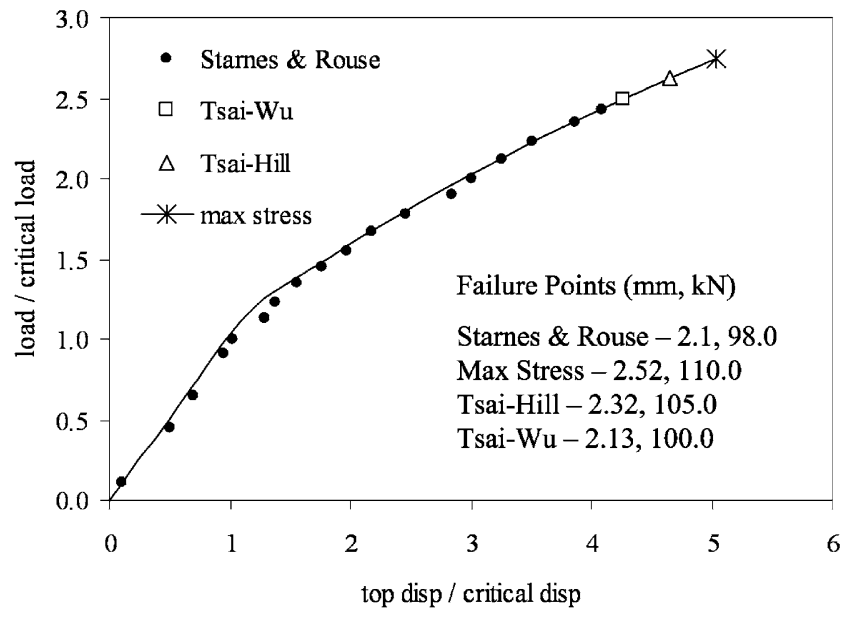

Fig. 55 Normalize load-deflection curve and failure of a C4/Graphite-epoxy panel

verse displacement at the center is chosen to be the imposed displacement degree of freedom where each imposed displacement increment equals 0.1 inch. In this example, both material and geometry nonlinearity are included.

The load vs. vertical displacement at point $A$ is given in Fig. 64 and is compared with the experimental results of Jofriet and McNiece [76] and the analysis results of Chung and Elisa [31].

The numerical results show that the response of slab is linear until the first crack has formed at the bottom surface at approximately $3.590 \mathrm{KN}(807 \mathrm{lb})$ of applied load. Be- 
Fig. 56 C4/Graphite-epoxy panel deformed shape and resultant stresses

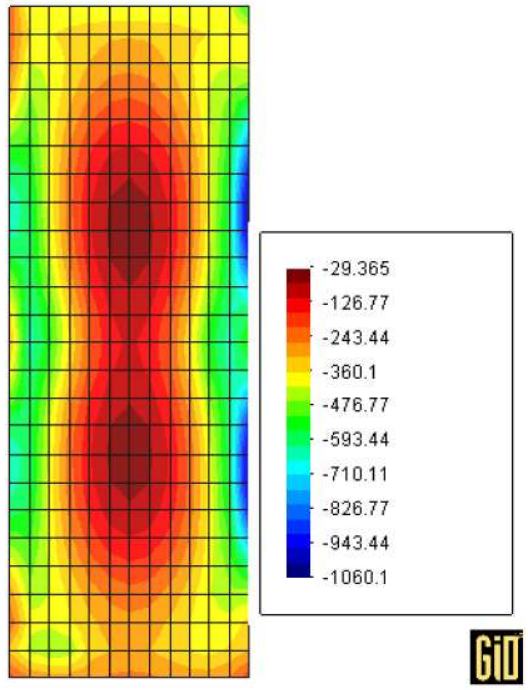

Resultant membrane stress $\mathrm{N}_{\mathrm{y}}$

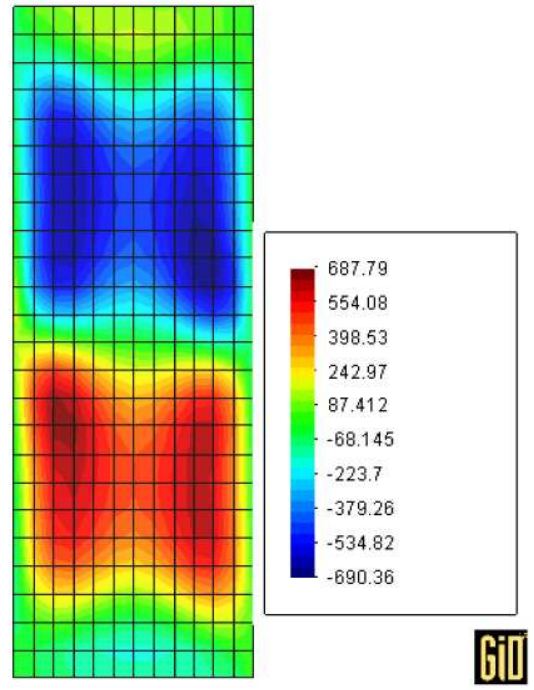

Resultant bending stress $\mathrm{M}_{\mathrm{x}}$

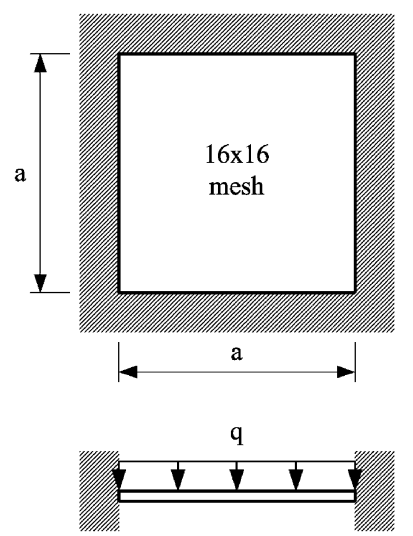

$\mathrm{E}_{1}=26.6 \mathrm{GPa}$

$\mathrm{E}_{2}=10.0 \mathrm{GPa}$

$\mathrm{G}_{12}=1.0 \mathrm{GPa}$

$v=0.23$

$\mathrm{a}=600 \mathrm{~mm}$

$\mathrm{h}=3.43 \mathrm{~mm}$

$\mathrm{Xt}=735.0 \mathrm{MPa}$

$\mathrm{Xc}=600.0 \mathrm{MPa}$

Fig. 57 Geometry and material properties of the e-glass plate under transverse load

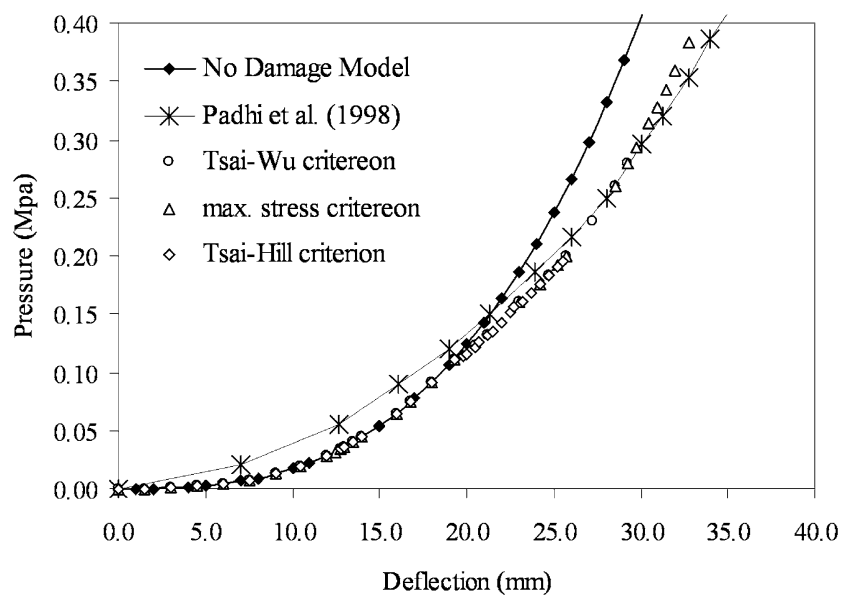

Fig. 58 Load-center deflection curves of the e-glass plate
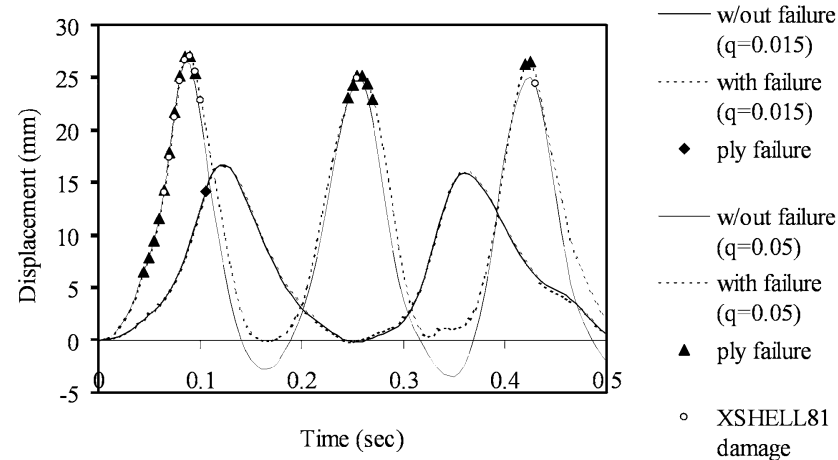

Fig. 59 Center deflection versus time of e-glass clamped plate with and without ply failure

yond this point, the slab exhibits the nonlinear behavior due to the progress of concrete cracking. The initial yielding of the reinforcing bar is predicted at $14.0 \mathrm{KN}(3,147 \mathrm{lb})$ from those three material models. The slab fails by yielding of reinforcing steel. As shown in Fig. 65b, the plastic strains in reinforcing steel spread over the centerline of the slab. This made the formation of the plastic hinge (yield line) and finally mechanism occurred. The unsymmetrical failure mode is caused by the different position of the steel layer ie; one of steel layer is placed over another one, this made the difference in moment capacity in the $x$ and $y$ directions of the slab. For EPF model, the difference of ultimate load between the predicted and experimental values is about $4 \%$ while the difference for the perfect plasticity and strain-hardening model are about $8 \%$ and 5\%, respectively. As can be seen, the numerical simulation captures the response accurately. In general, the FE predictions using present formulation are in close agreement with the corresponding experimental results. Therefore, the present material models used in the 
Fig. 60 Magnified plate deformation $(\times 10)$ at time $t=0.35 \mathrm{sec}$
Fig. 61 (a) Geometry and load of a concrete cylinder. (b) Finite element mesh

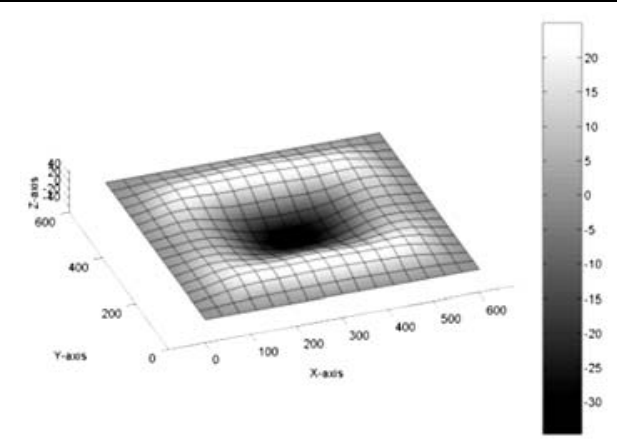

a) Undamaged Plate

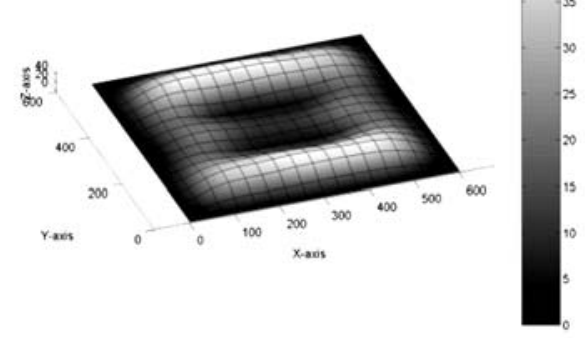

b) Plate with Damage

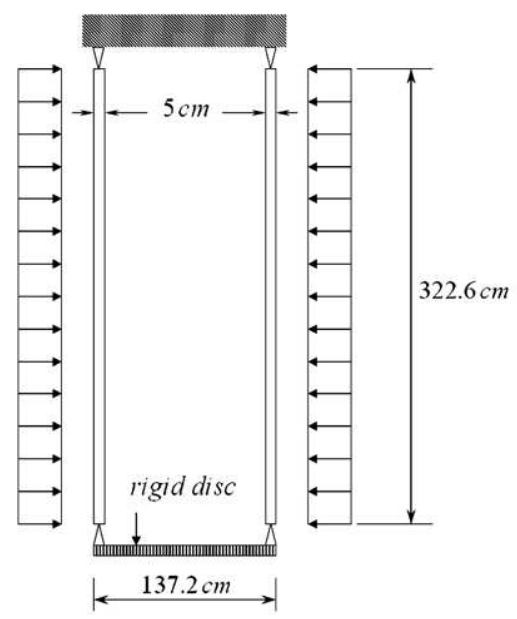

(a)

$$
\begin{array}{llll}
f_{c}^{\prime}=48.3 & M P a & E_{c}=25250 M p a & \varepsilon_{u}=0.0035 \\
f_{t}=4.83 & M P a & v=0.19 &
\end{array}
$$

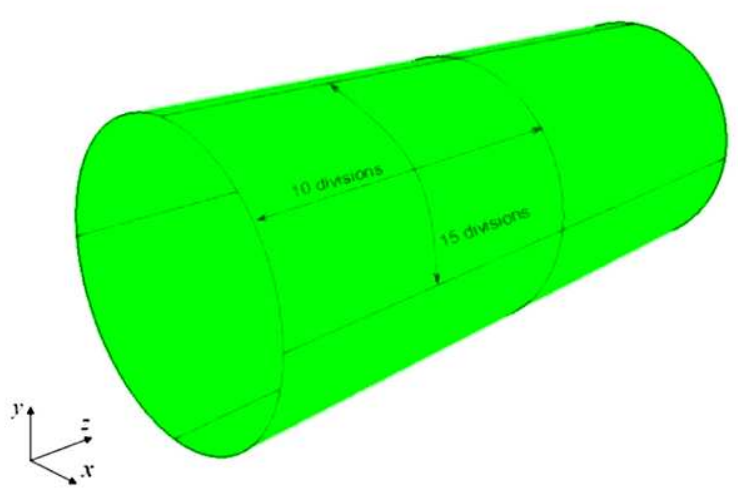

(b)

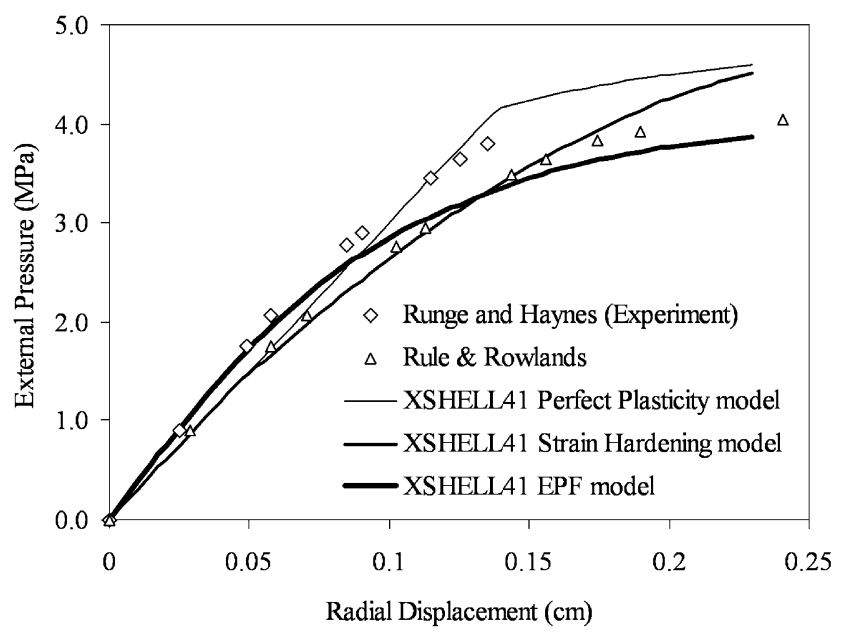

Fig. 62 Applied pressure versus mid length radial displacements

analysis is found to be capable of predicting the nonlinear behavior of the RC slab.
Duddeck Slabs In this example three corner supported slabs (S1, S2, S3) tested by Duddeck et al. [41] are studied. These slabs have various degrees of orthotropy of the steel reinforcement but total amount of reinforcement in slabs kept constant. This example has the advantage of welldefined support conditions where only transverse deflection is restrained at the corner support as shown in Fig. 67. All three slabs are tested under the same loading conditions of a concentrated load at the slab centre.

The material properties and the equivalent reinforcement layers for three slabs are given in Table 18 and the equivalent reinforcement layers for the three slabs are given in Table 19 in millimeters.

In the finite element analysis, by taking advantage of symmetry, it is only necessary to consider one quarter of the slab. A $9 \times 9$ mesh of the present shell element is used. The thickness of the slab is divided into 10 layers, and the reinforcement is represented by four of an equivalent smeared layer (two layers on the top and bottom sides).

The failure loads (expressed in kilo-Newton) obtained in the numerical analysis are listed in Table 20. The loaddeflection curves for the three slabs analyzed are compared 
Fig. 63 Geometry and finite element mesh of Mcniece slab

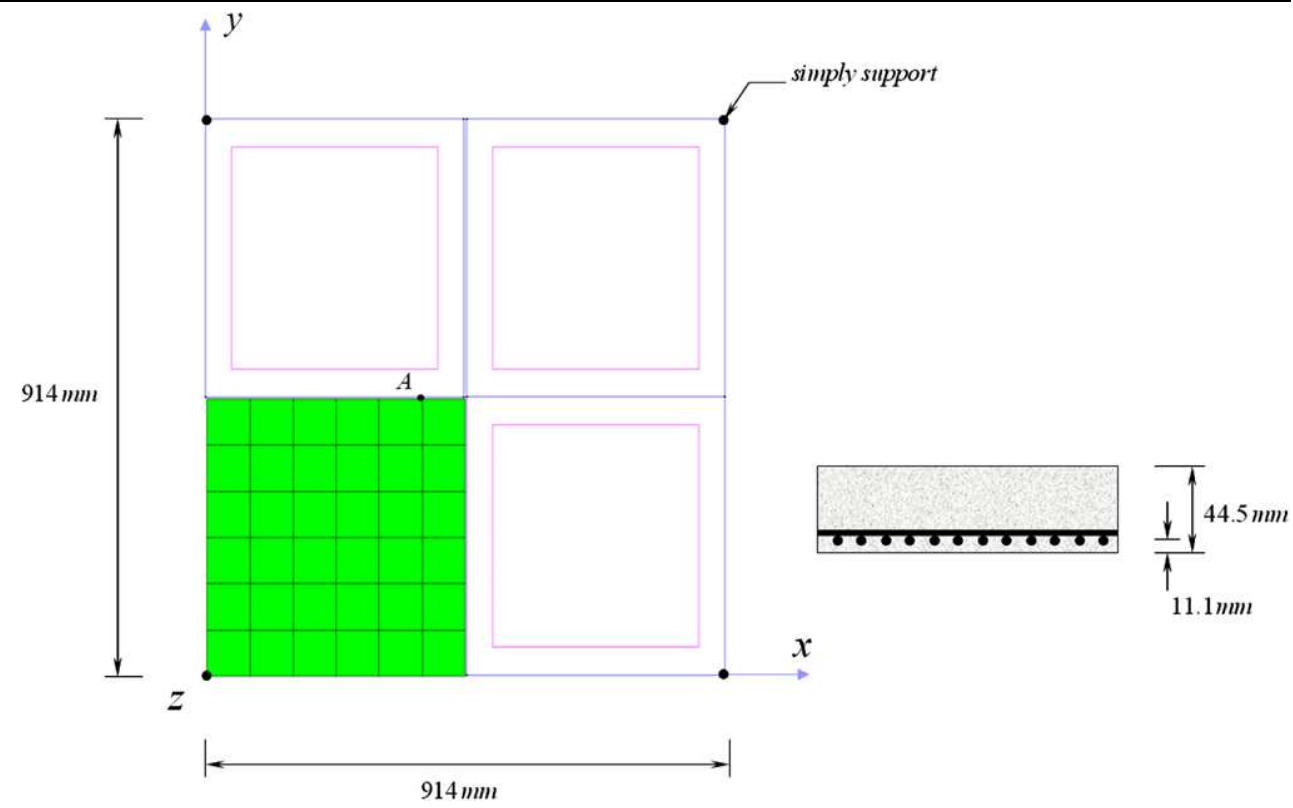

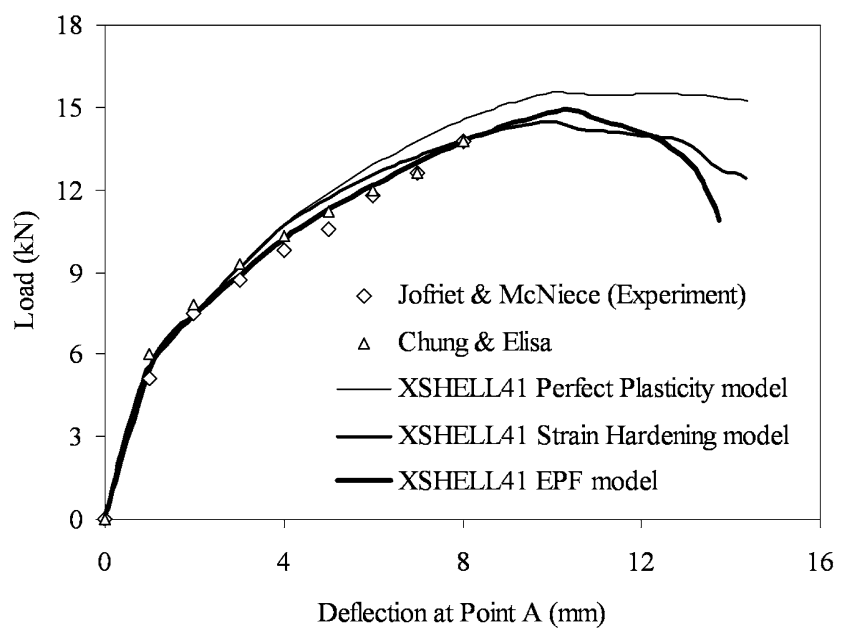

Fig. 64 Load-deflection curve of Mcniece slab

Table 17 Material properties of Mcniece slab

\begin{tabular}{ll}
\hline Concrete & Reinforcing Steel \\
\hline$E_{c}=28.6 \mathrm{GPa}$ & $E_{s 1}=200 \mathrm{GPa}$ \\
$f_{c}^{\prime}=37.9 \mathrm{MPa}$ & $E_{s 2}=0.0 \mathrm{GPa}$ \\
$f_{t}=3.10 \mathrm{MPa}$ & $f_{y}=413.45 \mathrm{MPa}$ \\
$v=0.15$ & $\rho_{x}=\rho_{y}=0.85 \%$ \\
$\varepsilon_{u}=0.0035$ & \\
\hline
\end{tabular}

in Figs. 68-70 and shown to match the experimental results. For each present material model, excellent agreement with the experiments is observed and is also found through the whole loading process. The slab S1 with isotropic reinforce- ment show smaller deflections and larger load carrying capacity, although the total amount of the steel is equal for all three slabs. In the analysis of slab S1, failure occurs by crushing of the concrete in the vicinity of the load, where the transverse shear stress effects are dominant. For the slabs S2 and $\mathrm{S} 3$, the load carrying capacity is found to be lower than slab S1, but these slabs show a ductile mode of failure. This is because the yield line has been completely formed at the ultimate load level as we can see in the plastic strain distribution of steel bar in Fig. 17. Although the slabs have different load carrying capacities, the cracking load of each slab are the same.

Parabolic Cylindrical Shell A parabolic cylindrical shell with variable thickness subjected to uniformly distributed pressure, which was tested by Hedgren [58], is analyzed with the present shell element. The shell is tested with end support diaphragms and free longitudinal edges. The shell geometry and zones with different layer patterns are shown in Fig. 74. The material properties are given in Tables 21, 22 and reinforcement patterns of each zone can be found in the reference [61]. Thirty-six finite elements are used to model one-quarter of shell as shown in Fig. 74, each of which is divided into 8 equal concrete layers. The shell is considered simply supported on its curved ends, assuming free longitudinal displacements and free rotation on a plane normal to the shell surface.

The design load of the shell is $0.358 \mathrm{~N} / \mathrm{cm}^{2}\left(0.52 \mathrm{lb} / \mathrm{in}^{2}\right)$. In the following presentation of results, the applied load is expressed in terms of a load factor $\lambda$, which is defined as the ratio of the applied load to the design load. In Fig. 77, the experimental load-vertical deflection curve at mid-span of the free edge is compared with the present results using 


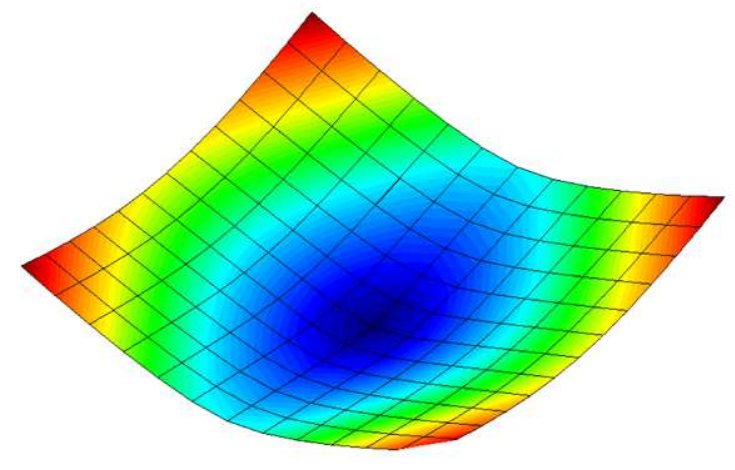

(a)

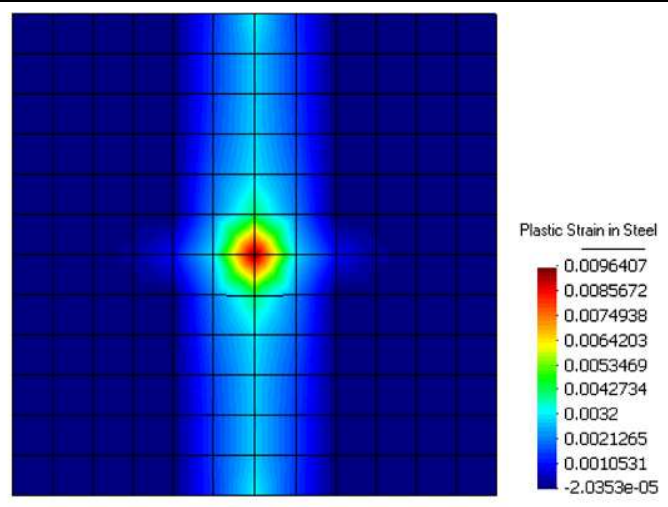

(b)

Fig. 65 (a) Deformation shape. (b) Plastic strain in the reinforcing steel (last analysis step)

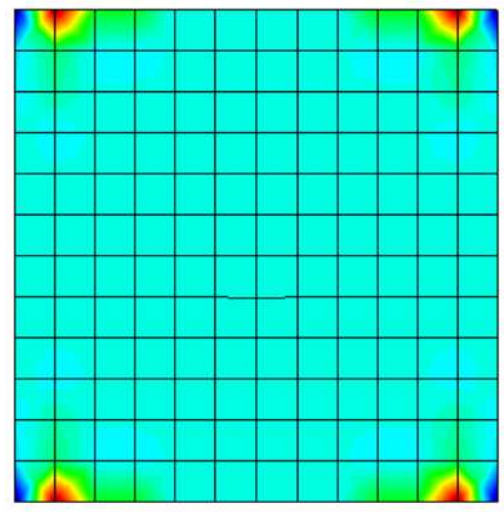

(a)

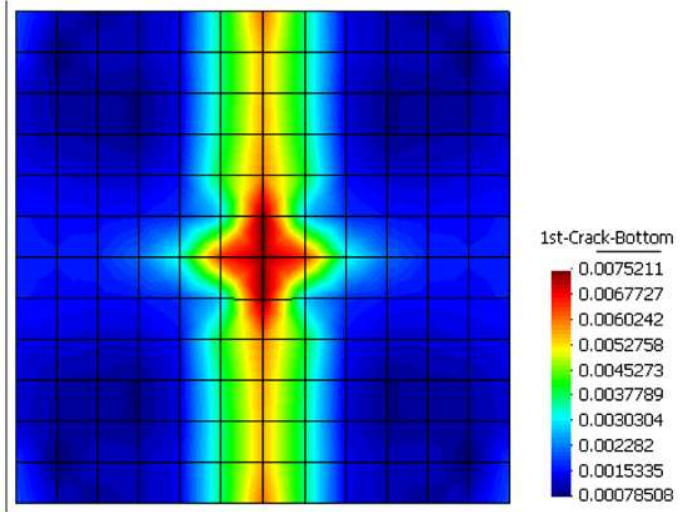

(b)

Fig. 66 (a) Crack strain in top surface. (b) Crack strain in bottom surface (last analysis step)

Fig. 67 Geometry and finite element mesh of Duddeck slabs

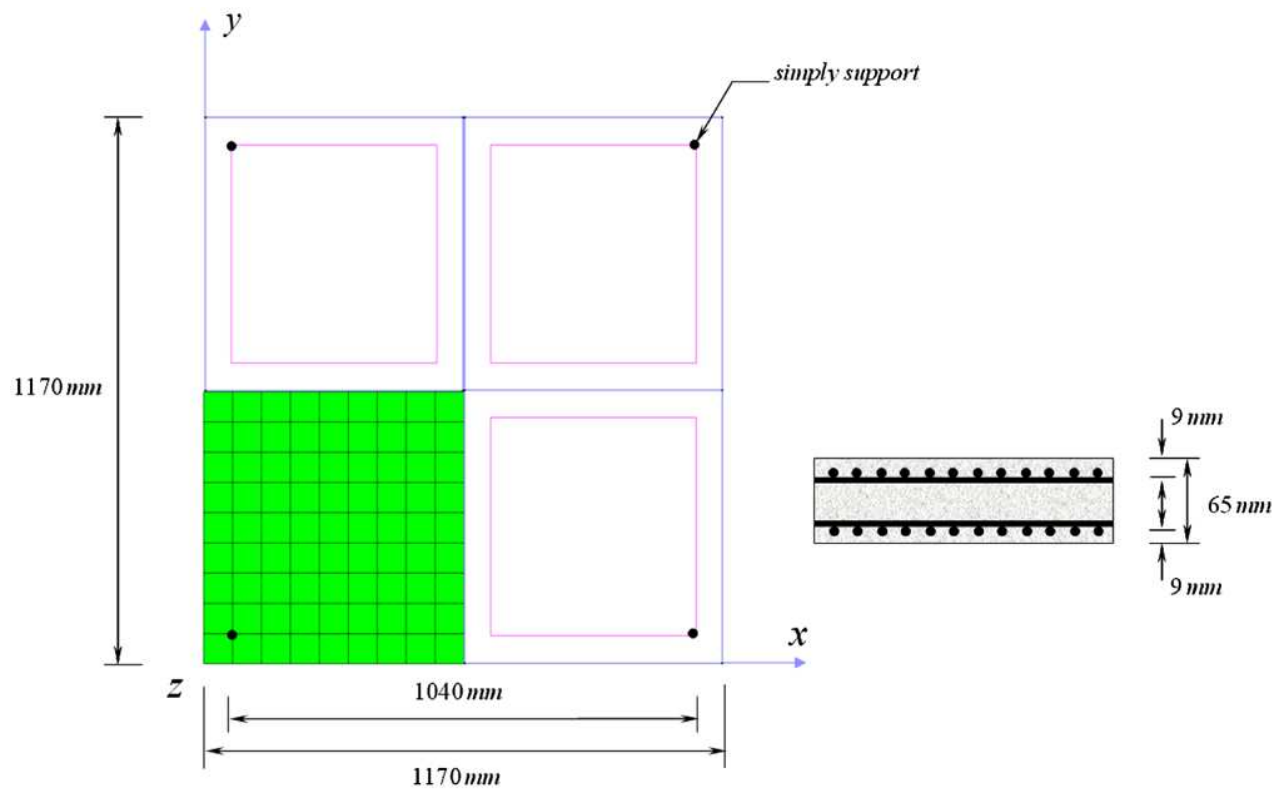


a linear and a nonlinear geometric model. The experimental curve is given only up to $\lambda=2.8$ and an experimental extrapolated curve presented in [49] is also included in Fig. 77. Using EPF model, the collapse load found for the nonlinear geometric analysis is $\lambda=4.39$, which agrees quite well with the reported experimental ultimate load, $\lambda=4.40$, and both are reached for identical collapse mechanisms. The geometrically linear analysis exhibits a very different response with softer behavior and lower load carrying capacity $(\lambda=3.25)$. Hedgren [58] pointed out the importance of nonlinear geo-

Table 18 Material properties of Duddeck slabs

\begin{tabular}{ll}
\hline Concrete & Reinforcing Steel \\
\hline$E_{c}=16.4 \mathrm{GPa}$ & $E_{s 1}=201 \mathrm{GPa}$ \\
$f_{c}^{\prime}=43.0 \mathrm{MPa}$ & $E_{s 2}=0.0 \mathrm{GPa}$ \\
$f_{t}=3.00 \mathrm{MPa}$ & $f_{y}=670 \mathrm{MPa}$ \\
$v=0.0$ & $\rho=1.82 \%$ \\
$\varepsilon_{u}=0.0035$ & \\
\hline
\end{tabular}

Table 19 Equivalent steel layer thickness of Duddeck slabs

\begin{tabular}{llllll}
\hline Slab & Top Layers & & & \multicolumn{2}{l}{ Bottom Layers } \\
No. & $X$-direction & $Y$-direction & & $X$-direction & $Y$-direction \\
\hline S1 & $0.193 \mathrm{~mm}$ & $0.193 \mathrm{~mm}$ & & $0.397 \mathrm{~mm}$ & $0.397 \mathrm{~mm}$ \\
S2 & $0.252 \mathrm{~mm}$ & $0.133 \mathrm{~mm}$ & & $0.520 \mathrm{~mm}$ & $0.273 \mathrm{~mm}$ \\
S3 & $0.283 \mathrm{~mm}$ & $0.103 \mathrm{~mm}$ & & $0.582 \mathrm{~mm}$ & $0.212 \mathrm{~mm}$ \\
\hline
\end{tabular}

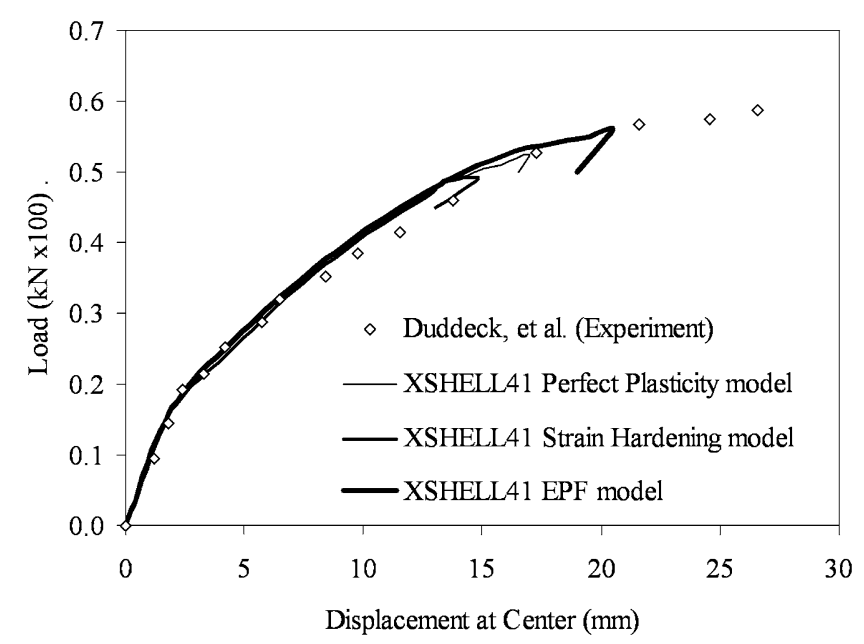

Fig. 68 Load-deflection curve of Duddeck slab S1 metric effects on the behavior of this shell. An increase of the shell stiffness and its load carrying capacity, due to the vertical uplift of the shell crown and the downward displacement of the free edges, is observed. An increase of lower arm of the internal forces is consequently obtained, when the deformed geometry is taken into consideration. The numerical failure mechanism can be clarified by reference to Fig. 79, where the plastic strains in the circumferential reinforcement are presented as contours at a load level $\lambda=4.39$. Positive values corresponding to the top layer and negative values refer to the bottom circumferential layer. The negative yield line (negative plastic strains) is completely

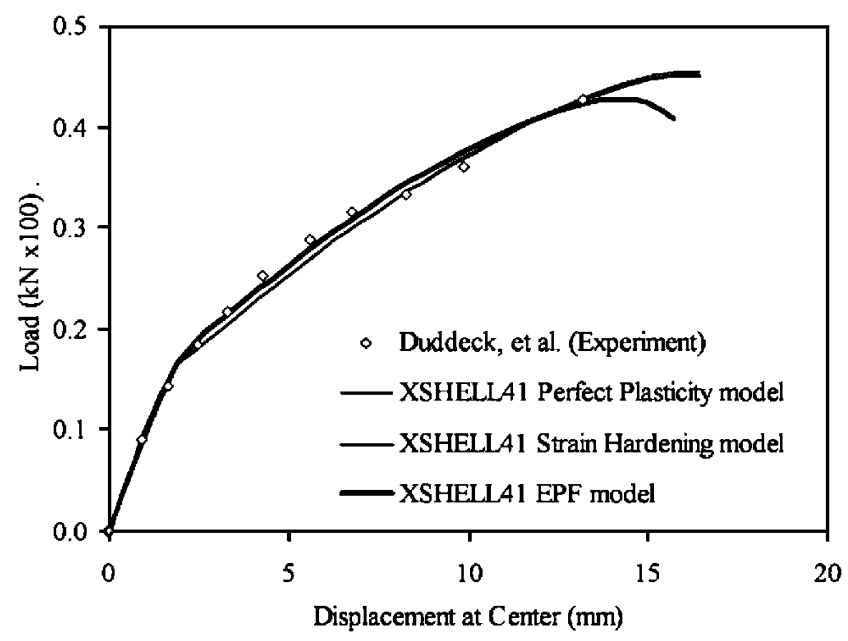

Fig. 69 Load-deflection curve of Duddeck slab S2

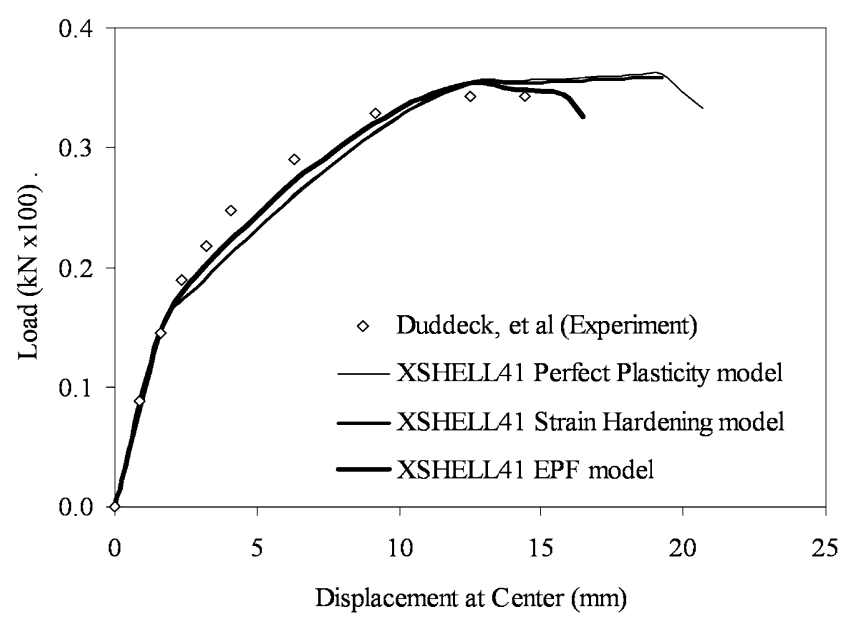

Fig. 70 Load-deflection curve of Duddeck slab S3
Table 20 The ultimate loads for Duddeck slabs

\begin{tabular}{llllc}
\hline Slab no. & Experimental & Perfect Plasticity model & Strain Hardening model & EPF model \\
\hline S1 & $61.06 \mathrm{KN}$ & $52.5 \mathrm{KN}$ & $49.1 \mathrm{KN}$ & $56.1 \mathrm{KN}$ \\
S2 & $43.46 \mathrm{KN}$ & $45.4 \mathrm{KN}$ & $45.1 \mathrm{KN}$ & $42.7 \mathrm{KN}$ \\
S3 & $34.25 \mathrm{KN}$ & $36.2 \mathrm{KN}$ & $35.9 \mathrm{KN}$ & $35.4 \mathrm{KN}$ \\
\hline
\end{tabular}


Fig. 71 Deformation shape of

Duddeck slabs. (a) S1. (b) S2.

(c) S3 (last analysis step)

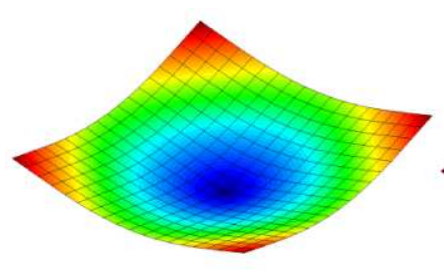

(a)

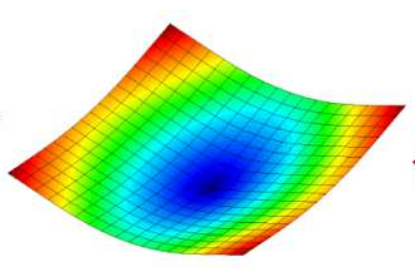

(b)

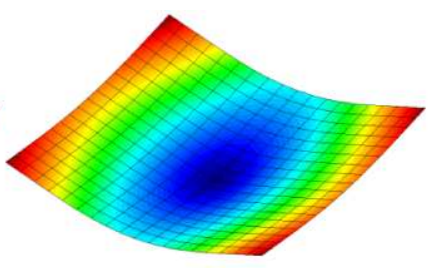

(c)

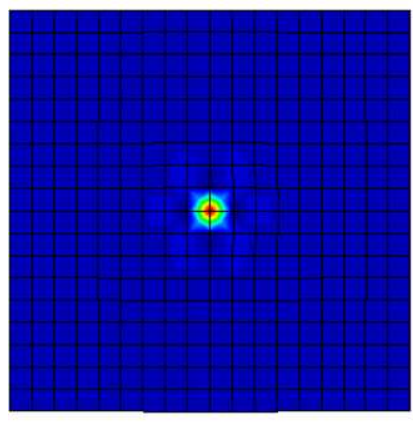

(a)

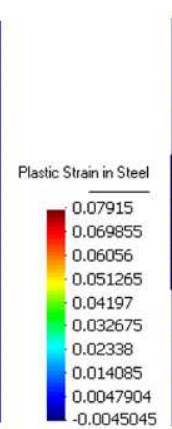

0.0045045

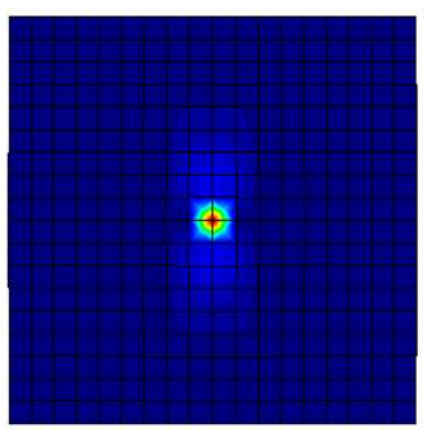

(b)

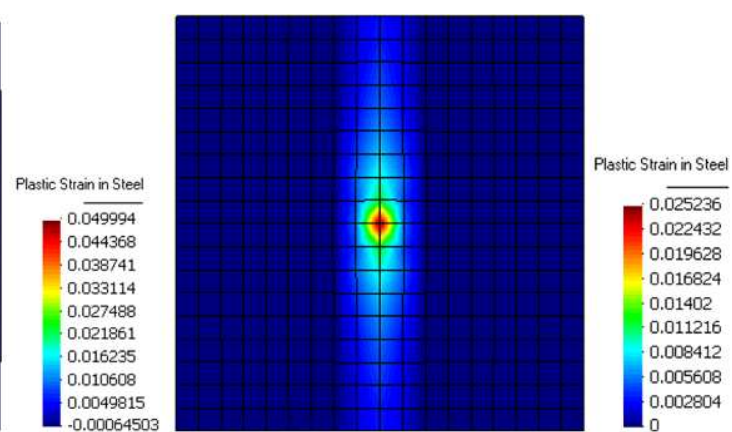

(c)

Fig. 72 Plastic strain in the reinforcing steel. (a) S1. (b) S2. (c) S3 (last analysis step)

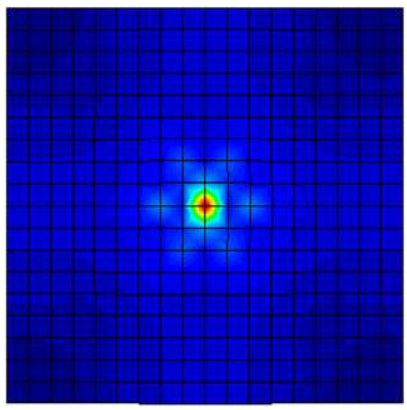

(a)

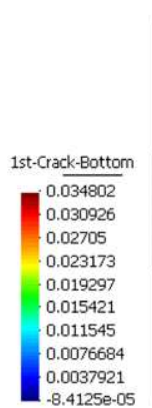

$-8.4125 \mathrm{e}-05$

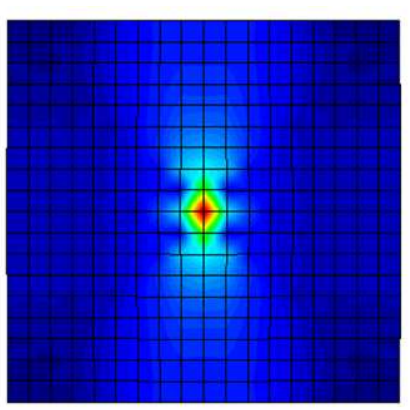

(b)

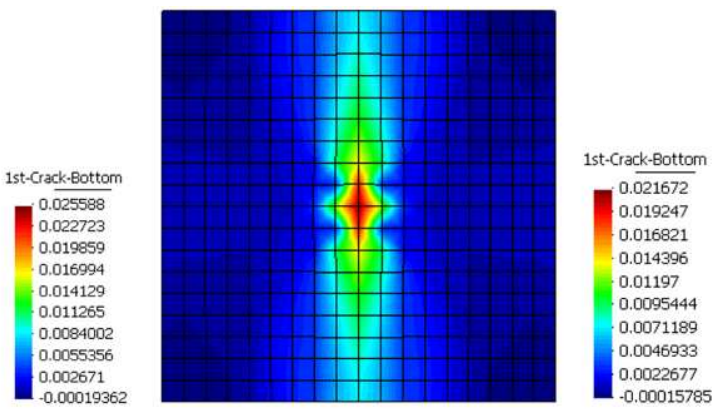

(c)

Fig. 73 Crack strain in bottom surface. (a) S1. (b) S2. (c) S3 (last analysis step)

Fig. 74 Geometry of a reinforced concrete parabolic cylindrical shell

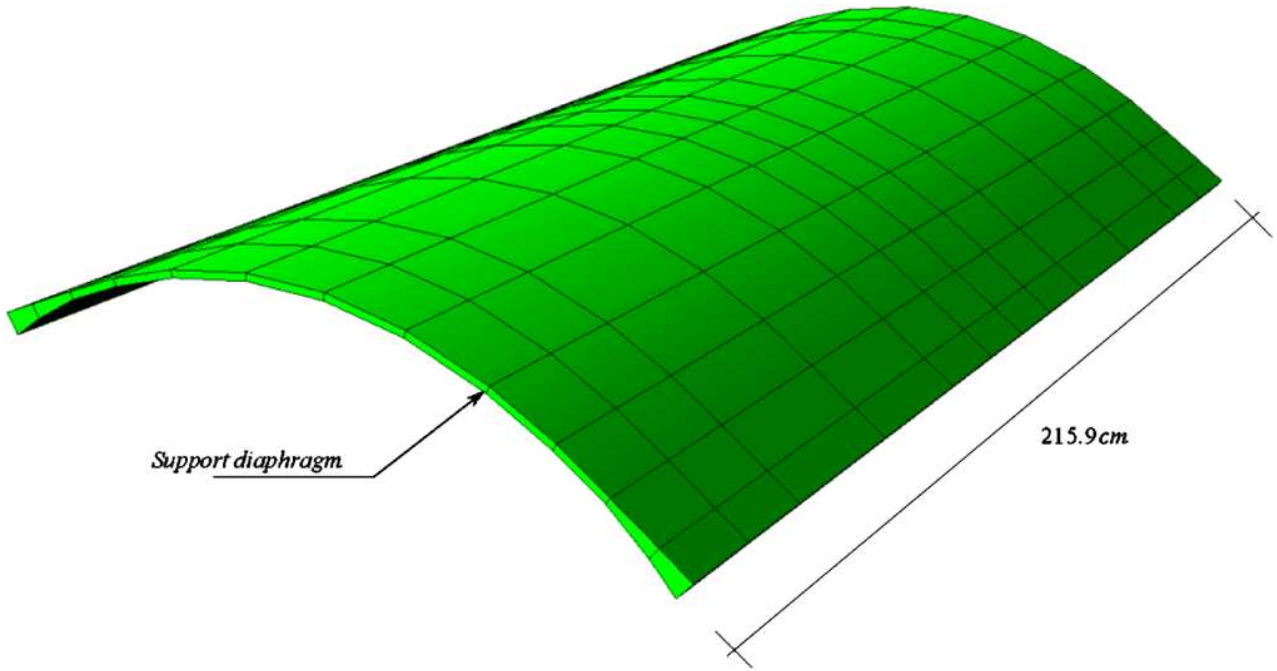


formed, whereas the two positive yield lines (positive plastic strains) are only partially formed along the edges.

Table 21 Material properties of parabolic cylindrical shell

\begin{tabular}{ll}
\hline Concrete & Reinforcing Steel \\
\hline$E_{c}=20.69 \mathrm{GPa}$ & $E_{s 1}=200 \mathrm{GPa}$ \\
$f_{c}^{\prime}=30.2 \mathrm{MPa}$ & $E_{s 2}=40.0 \mathrm{GPa}$ \\
$f_{t}=4.80 \mathrm{MPa}$ & $f_{y}=*$ see Table 22 \\
$v=0.145$ & \\
$\varepsilon_{u}=0.0035$ & \\
\hline
\end{tabular}

Table 22 Material properties of parabolic cylindrical shell-yield strengths

\begin{tabular}{ll}
\hline Designation & Yield Strength (MPa) \\
\hline$\# 3$ & 252.9 \\
$\# 4$ & 219.1 \\
$\# 9$ & 306.6 \\
\hline
\end{tabular}

\section{Concluding Remarks}

A nonlinear formulation based on the quasi-conforming technique, which includes geometric and material nonlinearity, was presented in this paper. The formulation was presented using an updated Lagrangian stress resultant, corotational approach. In the derivation of the geometric stiffness matrix, the trial basis function for the nonlinear strains is proposed to be made independent of its linear counterparts. Specific to the present formulation, it is proposed that a constant trial basis function be used as it has several benefits. First, while it is simple, it can easily provide solutions for buckling and post-buckling problems. Moreover, it does not degrade the nonlinear solution and does not require increased computational efforts. On the contrary, with the minimum amount of terms, it even reduces the number of computations. Lastly, it is shown to be robust and can be applied for various types of elements.

The proposed element passed the patch test with a maximum stress error of $0.075 \%$. For the linear analysis, poor
Fig. 75 Transverse section of a reinforced concrete parabolic cylindrical shell
Fig. 76 Finite element mesh and reinforcing steel pattern of a parabolic cylindrical shell

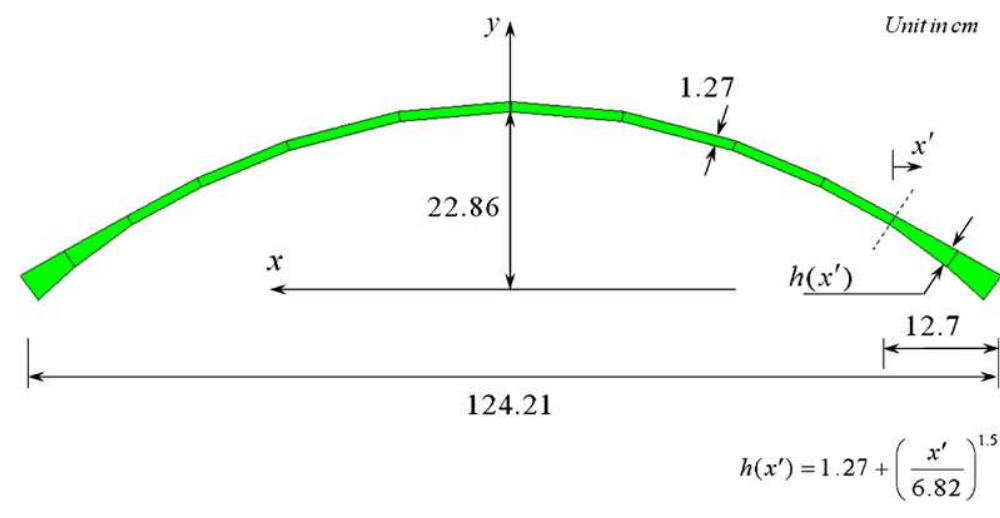




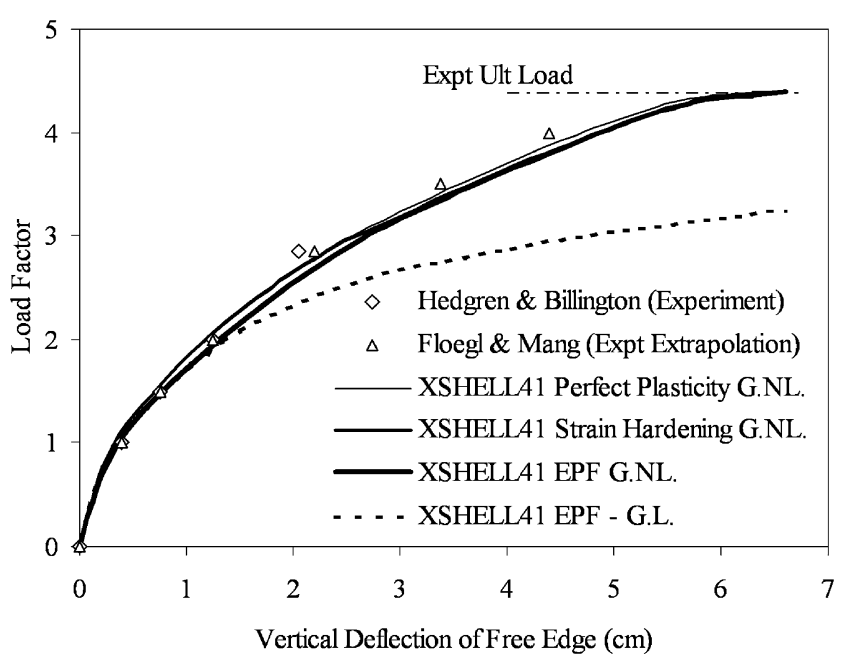

Fig. 77 Load-deflection curve of a reinforced concrete parabolic cylindrical shell

Fig. 78 Deformation shape of a parabolic cylindrical shell (last analysis step)

Fig. 79 Plastic strain distribution of the circumferential reinforcement (last analysis step) results were obtained in the in-plane shear when the mesh was rough and the aspect ratio was high, but it was shown to converge with sufficient mesh refinement. The element yielded very good results in bending and was free of shear locking. This is attributed to the higher-order string function that was used. The rate of convergence for bending problems was found to be faster for thin shells compared to thick shells.

For the nonlinear analysis, the element was shown to be useful in analyzing problems of large displacements and rotations. Very good results were also obtained for the geometrically nonlinear analysis, wherein, as discussed in the previous chapter, the nonlinear strains were derived from the full expression of the Green strain tensor and were assumed to have a constant distribution. The present formulation is also effective for the analysis of nonlinear material
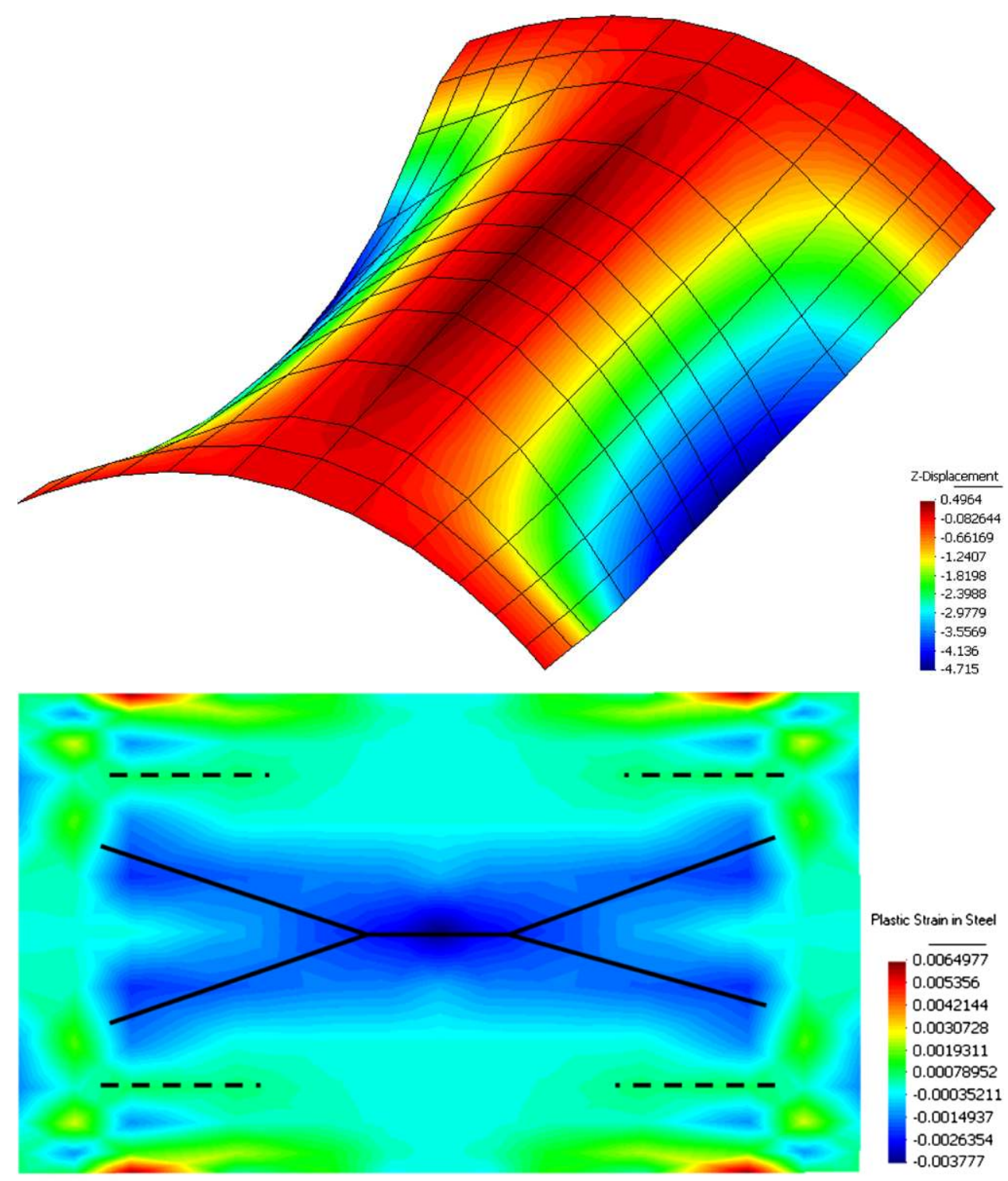
Fig. 80 Crack strain in top surface of a parabolic cylindrical shell (last analysis step)
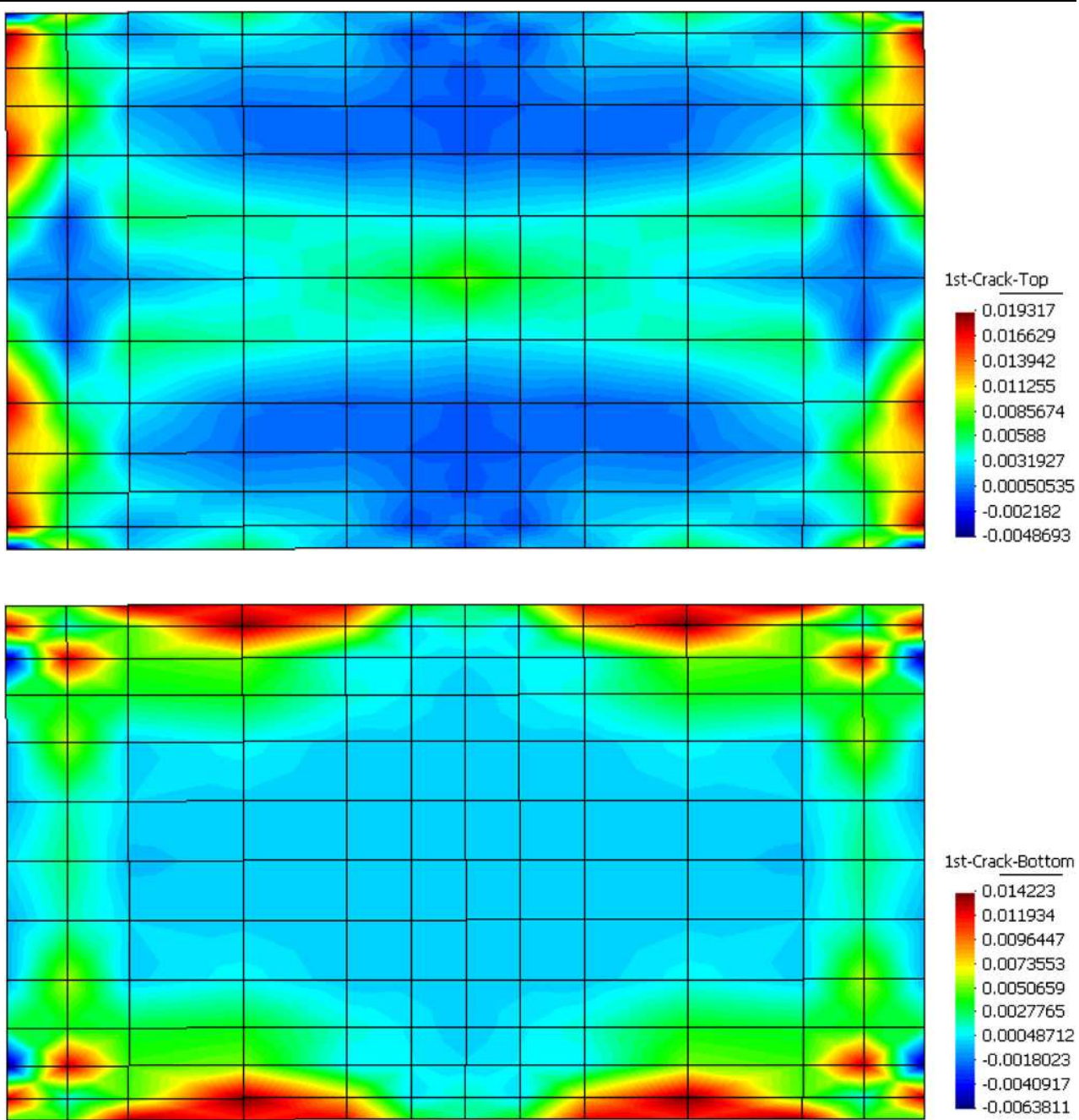

Fig. 81 Crack strain in bottom surface of a parabolic cylindrical shell (last analysis step) problems. The stresses occur directly at the nodes, and the spread of plasticity within the element is accounted for.

Acknowledgements This research was supported by a grant (06RNDD05) from Research and Development Program funded by Ministry of Construction \& Transportation of Korean government.

\section{References}

1. Agelidis N (1984) Buckling of stringer stiffened shells under axial and pressure loading, $\mathrm{PhD}$ thesis, Dept of Civil Engineering, Imperial College, London

2. Ahmad S, Irons BM, Zienkiewicz OC (1970) Analysis of thick and thin shell structures by curved finite elements. Int J Numer Methods Eng 2:419-451

3. Allman DJ (1984) A compatible triangular element including vertex rotations for plane elasticity analysis. Comput Struct 19:1-8

4. Argyris JH, Haase M, Malejannakis GA (1973) Natural geometry of surfaces with specific reference to the matrix displacement analysis of shells. Proc K Ned Akad Wet Ser B, 76(5)

5. Argyris JH, Scharpf DW (1968) The SHEBA family of shell elements for the matrix displacement method. Part I. Natural definition of geometry and strains. J R Aeronaut Soc 72:873-878
6. Argyris JH, Scharpf DW (1968) The SHEBA family of shell elements for the matrix displacement method. Part II. Interpolation scheme and stiffness matrix. J R Aeronaut Soc 71:878-883

7. Argyris JH, Scharpf DW (1969) The SHEBA family of shell elements for the matrix displacement method. Part III. Large displacements. J R Aeronaut Soc 73:423-426

8. Banbury A, Kelly DW, Jain LK (1999) A study of fastener pullthrough of composite laminates. Part 2. Failure prediction. Compos Struct 45:255

9. Basar Y, Ding Y (1997) Shear deformation models for largestrain shell analysis. Int J Solids Struct 34:1687-1708

10. Basar Y, Ding Y, Kratzig WB (1992) Finite-rotation shell elements via mixed formulation. Comput Mech 10:289-306

11. Bates ND (1987) The mechanics of thin walled structures with special reference to finite rotations. Doctors thesis, Imperial College of Science and Technology, London

12. Bazant ZP (2002) Concrete fracture models: testing and practice. Eng Fract Mech 69:165-205

13. Bazant ZP (2005) Energetic scaling of compression fracture and further applications to concrete, rock and composites. In: Scaling of structural strength, 2nd edn, pp 121-125

14. Bell JC, Elms D (1971) Partially cracked finite elements. J Struct Div, ASCE, 97(ST7)

15. Belytschko T, Bindeman LP (1991) Assumed strain stabilization of the 4-node quadrilateral with 1-point quadrature for nonlinear problems. Comput Methods Appl Mech Eng 88:311-340 
16. Belytschko T, Hseih J (1973) Nonlinear transient analysis with convected coordinates. Int J Numer Methods Eng 7:255-271

17. Belytschko T, Leviathan I (1994) Physical stabilization of the 4node shell element with one point quadrature. Comput Methods Appl Mech Eng 113:321-350

18. Belytschko T, Leviathan I (1994) Projection schemes for onepoint quadrature shell elements. Comput Methods Appl Mech Eng 115:277-286

19. Belytschko T, Tsay C (1983) A stabilization procedure for the quadrilateral plate element with one-point quadrature. Int $\mathrm{J} \mathrm{Nu}$ mer Methods Eng 19:405-419

20. Belytschko T, Wong BL, Stolarski H (1989) Assumed strain stabilization procedure for the 9 node Lagrange shell element. Int $\mathbf{J}$ Numer Methods Eng 28:385-414

21. Belytschko T, Wong BL, Chiang HY (1992) Advances in onepoint quadrature shell elements. Comput Methods Appl Mech Eng 96:93-103

22. Betsch P, Gruttmann F, Stein E (1996) A 4-node finite shell element for the implementation of general hyperelastic 3D-elasticity at finite strains. Comput Methods Appl Mech Eng 130:57-79

23. Brank B, Peric D, Damjanic FB (1997) On large deformation of thin elasto-plastic shells: implementation model for quadrilateral shell element. Int J Numer Methods Eng 40:689-726

24. Brank B, Korelc J, Ibrahimbegovic A (2002) Nonlinear shell problem formulation accounting for through-the-thickness stretching and its finite element implementation. Comput Struct 80:699717

25. Buechter N, Ramm E (1992) Shell theory versus degenerationa comparison in large rotation finite element analysis. Int $\mathrm{J} \mathrm{Nu}$ mer Methods Eng 34:39

26. Chang TY, Chen WF (1976) Extend NONSAP program for OTEC structural systems. ERDA Report COO-2682-7

27. Chen WJ (1983) Review of the quasi-conforming finite element with generalized variational principles. Acta Mech Sinica 4(1):129-135 (in Chinese)

28. Chen ACT, Chen WF (1975) Constitutive relations for concrete. J Eng Mech 101(4):465-481

29. Chen WF, Suzuki H, Chang TY (1980) Nonlinear analysis of concrete cylinder structures under hydrostatic loading. Comput Struct 12:559-570

30. Chen WJ, Tang LM (1981) Isoparametric quasi-conforming element. J Dalian Inst Technol 20(1):63-74 (in Chinese)

31. Chung W, Elisa DS (2005) Three-dimensional finite element modeling of composite girder bridges. Eng Struct 28:63-71

32. Crisfield MA, Moita GF, Jelenic G, Lyons LPR (1995) Enhanced lower-order element formulation for large strains. Comput Mech 17:62-73

33. Crisfield M, Peng X (1992) Efficient non-linear shell formulation with large rotations and plasticity. In: Owen DRJ (ed) Computational plasticity III: fundamentals and application. Pineridge, Swansea, pp 1979-1996

34. Darwin D, Pecknold DA (1974) Inelastic model for cyclic biaxial loading of reinforced concrete. Report SRS 409, Civil Engineering Studies, University of Illinois at Urbana Champaign, Urbana, Illinois

35. Darwin D, Pecknold DA (1977) Nonlinear biaxial stress-strain law for concrete. J Eng Mech Div 103:229-241

36. Dhatt G (1970) An efficient triangular shell element. AIAA J 8(11):2100-2102

37. Di Sciuva M (1987) An improved shear-deformation theory for moderately thick multilayered anisotropic shells and plates. ASME J Appl Mech 54:589-596

38. Djhani P (1977) Large deflection elasto-plastic analysis of discretely stiffened plates. PhD thesis, Dept of Civil Engineering, Imperial College, London
39. Doherty WP, Wilson EJ, Taylor TL (1969) Stress analysis of axisymmetric solids utilizing higher order quadrilateral finite elements. UC-SESM Report No. 69-3, Dept Civil Eng, Univ of California, Berkeley

40. Dong SB, Tso FKW (1972) On a laminated orthotropic shell theory including transverse shear deformation. ASME J Appl Mech 39:1091-1096

41. Duddeck H, Griebenow G, Shaper G (1978) Material and time dependent nonlinear behavior of cracked reinforced concrete slabs, nonlinear behavior of reinforced concrete spatial structures, vol 1. Preliminary Report IASS Symposium held in Darmstadt (eds G. Mehlhorn et al), pp 101-113

42. Dvorkin EN, Bathe KJ (1984) A continuum mechanics based four-node shell element for general non-linear analysis. Eng Comput 1:77-88

43. Dvorkin EN, Pantuso D, Repetto EA (1995) A formulation of the MITC4 element for finite strain elasto-plastic analysis. Comput Methods Appl Mech Eng 125:17-40

44. Eason TG, Ochoa OO (1996) Modeling progressive damage in composites: a shear deformable element for ABAQUS. Compos Struct

45. El-Abbasi N, Meguid SA (2000) A new shell element accounting for through-thickness deformation. Comput Methods Appl Mech Eng 189:841-862

46. Onate E (1992) Lectures on nonlinear finite element analysis of concrete shells. International Center for Numerical Methods in Engineering, Barcelona

47. Engelstad SP, Reddy JN, Knight NF Jr (1992) Post buckling response and failure prediction of graphite epoxy plates loaded in compression. AIAA J 30(8):2106-2113

48. Felippa CA, Haugen B (2005) A unified formulation of smallstrain co-rotational finite elements: I. Theory. Comput Methods Appl Mech Eng 194:2285-2335

49. Floegl H, Mang H (1981) On tension stiffening in cracked reinforced concrete slabs and shells considering geometric and physical nonlinearity. Ing Arch 51:215-242

50. Geubelle PH, Baylor JS (1998) Impact induced delamination of composites: a 2-D Simulation. Composites Part B 29:589-602

51. GID 7.1 (2002) Pre-postprocessor Software. International center for numerical methods in engineering. Barcelona, Spain, http:// gid.cimne.upc.es/

52. Gruttmann F, Stein E, Wriggers P (1989) Theory and numerics of thin elastic shells with finite rotations. Ing Arch 59:54-67

53. Guan Y, Tang LM (1992) A quasi-conforming nine-node degenerated shell finite element. Finite Elem Anal Des 11:165-176

54. Guan Y, Tang LM (1993) Nonlinear quasi-conforming finite element method. Acta Mech Sinica 9(3):269-276

55. Guan Y, Tang LM (1995) A geometrically non-linear quasiconforming nine-node quadrilateral degenerated solid shell element. Int J Numer Methods Eng 38:927-942

56. Guan Y, Zhang Z (1998) Quasi-conforming axisymmetric three node degenerated shell element. J Shanghai Jiaotong Univ 32(11):98-101

57. He DS, Tang LM (2001) The displacement function of quasiconforming element and its node error. Appl Math Mech 23(2):127-137

58. Hedgren AW, Billington DP (1967) Mortar model test on a cylindrical shell of varying curvature and thickness, ACI J 73-83

59. Hibbitt, Karlsson \& Sorensen, Inc (1994) ABAQUS example problems manual, Version 5.4

60. Hill R (1950) The mathematical theory of plasticity. Oxford University Press, New York

61. Hinton E, Owen DRJ (1984) Finite element software for plate and shells. Pineridge Press Limited, Swansea

62. Horrigmoe G, Bergan PG (1978) Nonlinear analysis of freeform shells by flat finite elements. Comput Methods Appl Mech Eng $16: 11$ 
63. Hu HT, Schnobrich WC (1990) Nonlinear analysis of cracked reinforced concrete. Struct J 87(2):199-207

64. Huang BZ, Atluri SN (1995) A simple method to follow post-buckling paths in finite element analysis. Comput Struct 57(3):477-489

65. Huang BZ, Shenoy VB, Alturi SN (1994) A quasi-conforming triangular laminated composite shell element based on a refined first-order theory. Comput Mech 13:295-314

66. Hueck U, Reddy BD, Wriggers P (1994) One the stabilization of the rectangular 4-node quadrilateral element. Commun Numer Methods Eng 10:555-563

67. Hughes TRJ, Brezzi F (1989) On drilling degrees of freedom. Comput Methods Appl Mech Eng 72:105-121

68. Hughes TJR, Taylor RL, Kanok-Nukulchai W (1977) A simple and efficient finite element for plate bending. Int J Numer Methods Eng 11:1529-1543

69. Hughes TJR, Brezzi F, Masud A, Harari I (1989) Finite elements with drilling degrees of freedom: theory and numerical evaluations. In: Gruber R, Periaux J, Shaw RP (eds) Proceedings of the fifth international symposium on numerical methods in engineering, vol 1. Springer, Berlin, pp 3-17

70. Hughes TJR, Masud A, Harari I (1995) Dynamic analysis with drilling degrees of freedom. Int J Numer Methods Eng 38(19):3193-3210

71. Ibrahimbegovic A (1994) Stress resultant geometrically nonlinear shell theory with drilling rotations-Part I. A consistent formulation. Comput Methods Appl Mech Eng 118:265-284

72. Ish J, Guttal R (1997) On the assumed strain formulation with selective polynomial order enrichment for P-version shells. Comput Struct 63(5):899-913

73. Javaherian H, Dowling PJ (1985) Large deflection elasto-plastic analysis of thin shells. Eng Struct 7:154-162

74. Jiang HY (1981) Derivation of higher precision triangular plate element by quasi-conforming element method. J Dalian Inst Technol 20(Suppl 2):21-28 (in Chinese)

75. Jiang HY (1984) Quasi-conforming model nonlinear finite element and others. PhD Dissertation, Dalian Institute of Technology, Dalian (in Chinese)

76. Jofriet JC, Mcniece GM (1971) Finite element analysis of reinforced concrete slabs. ASCE J Struct Div 97:785-806

77. Kanok-Nukulchai W (1979) A simple and efficient finite element for general shell analysis. Int J Numer Methods Eng 14:179

78. Kant T, Mallikarjuna (1991) Nonlinear dynamics of laminated plates with a higher order theory and $\mathrm{C} 0$ finite elements. Int $\mathrm{J}$ Nonlinear Mech 26:335-343

79. Kapania RK, Raciti S (1989) Recent advances in analysis of laminated beams and plates, Part I: shear effects and buckling. AIAA J 27:923-934

80. Kebari H, Cassel AC (1992) A stabilized 9-node non-linear shell element. Int J Numer Methods Eng 35:37-61

81. Kim KD (2003) A Co-rotational 8-node assumed strain element for large displacement elasto-plastic analysis of plates and shells. Struct Eng Mech Int J 15:199-223. 3

82. Kim KD, Lomboy GR (2006) A co-rotational quasi-conforming 4-node resultant shell element for large deformation elastoplastic analysis. Comput Methods Appl Mech Eng 195(4447):6502-6522

83. Kupfer HB, Gerstle KH (1973) Behavior of concrete under biaxial stresses. J Eng Mech Div, ASCE, EM4

84. Leicester RH (1968) Finite deformations of shallow shells. J Eng Mech Div ASCE 94:1409-1423

85. Lin CS (1973) Nonlinear analysis of reinforced concrete slabs and shells. Doctors thesis, Division of Structural Engineering and Structural Mechanics, University of California, Berkeley, UCSESM Report No 73-7
86. Lin FB, Bazant ZP, Chern JC, Marchertas AH (1987) Concrete model with normality and sequential identification. Comput Struct 26:1011-1025

87. Liu WK, Law ES, Lam D, Belytschko T (1986) Resultant-stress degenerated-shell element. Comput Methods Appl Mech Eng 55:259

88. Liu YX, Tang LM (1990) A triangular quasi-conforming finite element for transient dynamic analysis. Acta Mech Sinica 6(1):5963

89. Lomboy GR (2002) Non-linear dynamic analysis of composite shells with ply failure. School of Civil Engineering, Asian Institute of Technology, Pathum Thani, Thailand

90. Lu H, Tang LM, Wang X (1993) The upper bound on the collapse load of plate bending by using a quasi-conforming element and the Monte-Carlo method. Finite Elem Anal Des 13(1):65-73

91. Ma H (1990) Development of a geometrically nonlinear shell element by assumed strain methods. Doctoral dissertation, Asian Institute of Technology, Thailand

92. MacNeal RH, Harder RL (1985) A proposed standard set of problems to test finite element accuracy. Finite Elem Anal Des $1: 3-20$

93. Maekawa K, Pimanmas A, Okamura H (2003) Nonlinear mechanics of reinforced concrete. Spon, London

94. Moita GF, Crisfield MA (1996) A finite element formulation for 3-D continua using the co-rotational technique. Int J Numer Methods Eng 39:3775-3792

95. Moy SS, Shenoi RA, Allen HG (1996) Strength and stiffness of fiber reinforced plastic plates. Proc Inst Civil Eng 116:204-220

96. Ngo D, Scordelis AC (1967) Finite element analysis of reinforced concrete beams. J Am Concr Inst 64(3):152-163

97. Noor AK, Mathews MD (1975) Shear flexible finite element models of laminated composite plates. NASA TN D-8044

98. Novozhilov VV (1963) Foundation of non-linear theory of elasticity. Graylock, Rochester

99. Ochoa OO, Engblom JJ (1987) Analysis of progressive failure in composites. Comp Sci Technol 28:87-102

100. Oliver J, Linero DL, Huespe AE, Manzoli OL (2008) Twodimensional modeling of material failure in reinforced concrete by means of a continuum strong discontinuity approach. Comput Methods Appl Mech Eng 197:332-348

101. Oller S, Onate E, Oliver J, Lubliner J (1990) Finite element nonlinear analysis of concrete structures using a "plastic-damage model". Eng Fract Mech 35:219-231

102. Owen DRJ, Figueiras JA (1984) Ultimate load analysis of reinforced concrete plates and shells including geometric non linear effects. In: Hinton E, Owen DRJ (eds) Finite element software for plates and shells. Pineridge, Swansea

103. Padhi GS, Shenoi RA, Moy SSJ, Hawkins GL (1998) Progressive failure and ultimate collapse of laminated composite plates in bending. Compos Struct 40:277-291

104. Park T, Kim KD, Han S (2006) Linear static and dynamic analysis of laminated composite plates and shells using a 4-node quasiconforming shell element. Compos Part B, Eng 37(2-3):237248

105. Pawsey SE, Clough RW (1971) Improved numerical integration of thick shell finite elements. Int J Numer Methods Eng 3:575586

106. Peng X, Crisfield MA (1992) A consistent co-rotational formulation for shell using the constant stress/constant moment triangle. Int J Numer Methods Eng 35:1829-1847

107. Peric D, Owen DRJ (1991) The morley thin shell finite element for large deformation problems: simplicity versus sophistication. In: Bicanic N (ed) Proc int conf on nonlinear engineering computations. Pineridge, Swansea, p 121

108. Pian THH (1964) Derivation of element stiffness matrices by assumed stress distributions. AIAA J 2(7):1333-1336 
109. Polak MA, Vecchio FJ (1993) Nonlinear analysis of reinforced concrete shells. J Struct Eng ASCE 119(12):3439-3462

110. Polak MA, Vecchio FJ (1994) Reinforced concrete shell elements subjected to bending and membrane loads. ACI Struct J 91(2):261-268

111. Pucha NS, Reddy JN (1984) A mixed shear flexible finite element for the analysis of laminated plates. Comput Methods Appl Mech Eng 44:213-227

112. Qin QH (1995) Geometrically nonlinear analysis of shells by the variational approach and an efficient finite element formulation. Comput Struct 55(4):727-733

113. Ramm E (1982) The Riks/Wempner approach. An extension of the displacement control method in nonlinear analysis. In: Hinton E, Owen DRJ, Taylor C (eds) Recent advances in non-linear computational mechanics. Pineridge, Swansea, pp 63-86

114. Reddy JN (1983) Geometrically non-linear transient analysis of laminated composite plates. AIAA J 21:621-629

115. Reddy JN (1987) A generalization of two-dimensional theories of laminated composite plates. Commun Appl Numer Methods 3:173-180

116. Reddy JN (1990) A general third-order nonlinear theory of plates with moderate thickness. J Nonlinear Mech 25:677-686

117. Reddy JN (2004) Mechanics of laminated composite plates and shells: theory and analysis, 2nd edn. CRC, Boca Raton, pp 567670

118. Reddy JN, Chandrashekhara K (1985) Geometrically nonlinear transient analysis of laminated doubly curved shells. Int J Nonlinear Mech 20:79-90

119. Reddy JN, Liu CF (1985) A higher-order shear deformation theory of laminated elastic shells. Int J Eng Sci 23:319

120. Reddy JN, Robbins Jr. DH (1994) Theories and computational models for composite laminates. ASME Appl Mech Rev 47:147169

121. Reddy YSN, Moorthy C.M.D. and Reddy JN (1995) Non-linear progressive failure analysis of laminated composite plates. Int $\mathbf{J}$ Nonlinear Mech 30:629-649

122. Roehl D, Ramm E (1996) Large elasto-plastic finite element analysis of solids and shells with the enhanced assumed strain concept. Int J Solids Struct 33:3215-3237

123. Rolfes R, Rohwer K (1997) Improved transverse shear stress in composite finite element based on first order shear deformation theory. Int J Numer Methods Eng 40:51-60

124. Rule WK, Rowlands RE (1989) A simple orthotropic elasticitybased constitutive model for reinforced concrete. Exp Mech 448454

125. Runge KH, Haynes HH (1978) Experimental implosion study of concrete structures, in: Proceedings of the eighth congress of the federation international de la precontrainte, London, England, May 1978

126. Sabir AB, Lock AC (1973) The application of finite elements to large deflection geometrically non-linear behaviour of cylindrical shells. In: Brebbia CA, Totenham HN (eds) Variational method in engineering. Southampton University Press, Southampton, pp $66-75$

127. Saigal S, Kapania RK, Yang TY (1986) Geometrically nonlinear finite element analysis of imperfect shells. J Compos Mater 20:197

128. Sandhu RS, Gallo RL, Sendeckyj GP (1982) Initiation and accumulation of damage in composite laminates. In: Daniels IM (ed) Composite materials: testing and design, sixth conference, pp 163-182

129. Schonobrich WC (1976) Behavior of reinforced concrete predicted by finite element method. In: Proceeding of the second national symposium on computerized structural analysis and design. George Washington University, Washington. March
130. Scordelis AC (1972) Finite element analysis of reinforced concrete structures. In: Proceeding of the specialty conference on finite element methods in civil engineering, Montreal

131. Scordelis AC, Chan EC (1987) Nonlinear analysis of reinforced concrete shell. In: Computer applications in concrete technology, ACI SP-98. Am Concr Inst, Detroit, pp 25-57

132. Shi G, Voyiadjis G (1991) Geometrically nonlinear analysis of plates by assumed strain element with explicit tangent stiffness matrix. Comput Struct 41(4):757-763

133. Shi G, Voyiadjis G (1991) Efficient and accurate four-node quadrilateral $\mathrm{C} 0$ plate bending element based on assumed strain fields. Int J Numer Methods Eng 32(5):1041-1055

134. Shi G, Voyiadjis G (1991) A simple C0 quadrilateral thick/thin shell element based on the refined shell theory and the assumed strain fields. Int J Solids Struct 27(3):283-298

135. Shi G, Voyiadjis G (1992) A simple non-layered finite element for the elasto-plastic analysis of shear flexible plates. Int J Numer Methods Eng 33(1):85-99

136. Simo JC (1993) On a stress resultant geometrically exact shell model. Part VII: shell intersections with 5/6-DOF finite element formulations. Comput Methods Appl Mech Eng 108:319-339

137. Simo JC, Armero F (1992) Geometrically non-linear enhanced strain mixed methods and the method if incompatible modes. Int J Numer Methods Eng 33:1413-1449

138. Simo JC, Fox DD (1989) On a stress resultant geometrically exact shell model. Part I: formulation and optimal parameterization. Comput Methods Appl Mech Eng 72:267-304

139. Simo JC, Fox DD, Rifai MS (1990) On a stress resultant geometrically exact shell model. Part III: computational aspects of the nonlinear theory. Comput Methods Appl Mech Eng 79:2170

140. Simo JC, Kennedy JG (1992) On a stress resultant geometrically exact shell model. Part V: nonlinear plasticity: formulation and integration algorithms. Comput Methods Appl Mech Eng 96:133-171

141. Simo JC, Rafai MS (1990) A class of mixed assumed strain methods and the method of incompatible modes. Int J Numer Methods Eng 29:1595-1638

142. Simo JC, Fox DD, Hughes TJR (1992) Formulations of finite elasticity with independent rotations. Comput Methods Appl Mech Eng 95:277-288

143. Simo JC, Rifai MS, Fox DD (1992) On a stress resultant geometrically exact shell model. Part VI: conserving algorithms for non-linear dynamics. Int J Numer Methods Eng 34:117-164

144. Simo JC, Fox DD, Rifai MS (1989) On stress resultant geometrically exact shell model. Part II: the linear theory; computational aspects. Comput Methods Appl Mech Eng 73:53-92

145. Spilker RL, Engleman BE (1986) Hybrid-stress isoparametric element for moderately thick and thin multilayered plates. Comput Methods Appl Mech Eng 56:339-361

146. Spottswood SM, Palazotto AN (2001) Progressive failure analysis of a composite shell. Compos Struct 53(1):117-131

147. Starnes JH, Rouse M (1981) Post buckling and failure characteristics of selected flat rectangular graphite-epoxy plates loaded in compression. AIAA Paper No 81-0543

148. Tan XG, Vu-Quoc L (2005) Efficient and accurate multilayer solid-shell element: nonlinear materials at finite strain. Int $\mathrm{J} \mathrm{Nu}$ mer Methods Eng 63(15):2124-2170

149. Tang LM, Chen WJ, Liu YX (1980) Quasi-conforming elements for finite element analysis. J Dalian Inst Technol 10(2):19-35 (in Chinese)

150. Tang LM, Chen WJ, Liu YX (1984) Formulation of quasiconforming element and $\mathrm{Hu}-$ Washizu principle. Comput Struct 19(1-2):247-250

151. Tang LM, Chen WJ, Zhou JQ (1988) A multi-variable quasiconforming quadrilateral element. J Comput Struct Mech Appl 5:1-6 (in Chinese) 
152. Tang LM, Liu YX (1985) Quasi-conforming element techniques for penalty finite element methods. Finite Elem Anal Des 1:2533

153. Tang LM, Lu HX, Chen WJ, Liu YX (1980) Quasi-conforming element technique for the finite element method. Numerical methods for engineering. In: Proceedings of the second international congress, Paris, France, pp 565-573

154. Taylor RL (1987) Finite element analysis of linear shell problems. In: Whiteman JR (ed) Proc of the mathematics in finite elements in applications. Academic, New York, pp 191-203

155. Taylor RL (1987) Finite element analysis of linear shell problems. In: Whiteman JR (ed) Proc of the mathematics in finite elements in applications. Academic, New York, pp 191-203

156. Taylor RL, Beresford PJ, Wilson EL (1976) A non-conforming element for stress analysis. Int J Numer Methods Eng 10:12111219

157. Timoshenko S, Woinowsky-Krieger S (1959) Theory of plates and shells. McGraw-Hill, Singapore

158. Toson S, Zabaras N (1991) Finite element analysis of progressive failure in laminated composite plates. Comput Struct 38(3):361376

159. Tsai SW (1968) Strength theories in filament structures. In: Schwartz RT, Schwartz HS (eds) Fundamental aspects of fiber reinforced plastic composites. Wiley Interscience, New York. Chap 1

160. Vecchio FJ, Collins MP (1986) The modified compression-field theory for reinforced concrete elements subjected to shear. J Am Concr Inst 83(2):219-231

161. Voyiadjis GZ, Shi G (1992) Nonlinear post-buckling analysis of plates and shallow shells by four-noded strain element. AIAA J 30:1110-1116

162. Voyiadjis GZ, Woelke P (2006) General non-linear finite element analysis of thick plates and shells. Int J Solids Struct 43(78):2209-2242

163. Vu-Quoc L, Tan XG (2003) Optimal solid shells for nonlinear analyses of multilayer composites. Part I: statics. Comput Methods Appl Mech Eng 192:975-1016

164. Vu-Quoc L, Tan XG (2003) Optimal solid shells for nonlinear analyses of multilayer composites. Part II: dynamics. Comput Methods Appl Mech Eng

165. Wang X, Jiang HY, Lee LHN (1991) Finite deformation of a shell element for problems of sheet metal forming. Comput Mech 7:397-411

166. Webb SE, Dowling PJ (1980) Large-deflection elasto-plastic behaviour of discretely stiffened plates. Proc Inst Civil Eng, Part 23:75-401

167. Whitney JM (1969) Bending-extensional coupling in laminated plates under transverse loading. J Compos Mater 3:20-28

168. Whitney JM (1970) The effect of boundary conditions on the response of laminated composites. J Compos Mater 3:192-203

169. Whitney JM (1973) Shear correction factors for orthotropic laminates under static loads. J Appl Mech Trans ASME 40:302-304
170. Whitney J, Pagano N (1970) Shear deformation in heterogeneous anisotropic plates. J Appl Mech 37:1031

171. Wiggenraand JFM, Zhang X, Davies GAO (1999) Impact damage prediction and failure analysis of heavily loaded, bladestiffened composite wing panels. Compos Struct 45:81-103

172. Wilson EL, Taylor RL, Doherty WP, Ghaboussi J (1973) Incompatible displacement models. In: Fenves SJ et al (eds) Numerical and computer models in structural mechanics. Academic, New York, pp 43-57

173. Woelke P, Voyiadjis GZ, Perzyna P (2006) Elasto-plastic finite element analysis of shells with damage Due to microvoids. Int J Numer Methods Eng 68:338-380

174. Wriggers P, Gruttmann F (1993) Thin shell with finite rotations formulated in biot stresses: theory and finite element formulation. Int J Numer Methods Eng 36:2049-2071

175. Wriggers P, Hueck U (1996) A formulation of the QS6 element for large elastic deformation. Int J Numer Methods Eng 39:14371454

176. Wu YC, Yang TY (1987) Free and forced nonlinear dynamics of composite shell structures. J Compos Mater 21:898-910

177. Yang HTY, Saigal S, Liaw DG (1990) Advances of thin shell finite elements and some applications. Version I. Comput Struct 35(4):481-504

178. XFINAS 2.0 (2005) Asian Institute of Technology \& Konkuk University, Korea, http://www.xfinas.com/

179. Yang HTY, Saigal S, Masud A, Kapania RK (2000) A survey of recent shell finite elements. Int J Numer Methods Eng 47:101127

180. Zaghloul SA, Kennedy JB (1975) Non-linear analysis of unsymmetrically laminated plates. J Eng Mech 3:169-186

181. Zhang HQ (1982) The generalized patch test for multiple sets of functions and 12-parameters quasi-conforming element. J Dalian Inst Technol 21(3):11-19 (in Chinese)

182. Zhang Y (1982) Buckling and post buckling behavior of generally layered composite panels. $\mathrm{PhD}$ thesis, Imperial College, London

183. Zhang HQ (1983) The generalized patch test and 9-parameters quasi-conforming element. In: Kang F, Lions JL (eds) Proceedings of the Sino-France symposium on finite element methods. Science, Gordon and Breach, New York, pp 566-583

184. Zhang HQ, Wang M (1985) Finite element approximations with multiple sets of functions and quasi-conforming elements for plate bending problems. Appl Math Mech 6(1):41-52

185. Zhao Z, Chen W (1995) New finite element model for analysis of Kirchhoff plate. Int J Numer Methods Eng 38(7):1201-1214

186. Zhu G, Wang H (1989) Quasi-conforming penalty FEM for large deflection of composite laminated plate. Acta Mater Compos Sinica 6:39-47 (in Chinese)

187. Zienkiewicz OC, Taylor RL, Too JM (1971) Reduced integration technique in general analysis of plates and shells. Int $\mathrm{J}$ Numer Methods Eng 3:275-290 